\title{
LIVING WELL WITH CHRONIC PAIN: A CLASSICAL GROUNDED THEORY
}

Bronwyn F Lennox Thompson

A thesis submitted in fulfilment of the requirements for the Degree of Doctor of Philosophy in Health Sciences in the University of Canterbury

University of Canterbury 
Living well with chronic pain: A classical grounded theory 


\begin{abstract}
Chronic pain is a public health problem that is likely to increase as the population ages, and has few effective treatments. Although viewed by many as profoundly distressing and disabling, there are a surprising number of people (approximately $30 \%$ ) who cope well with their chronic pain and do not continue to seek treatment. There is little theory to explain how and why these individuals manage their pain well. This means there is limited knowledge about the approaches used by people who cope well and whether these strategies could help those who have more difficulty.

This thesis presents a substantive grounded theory of living well with chronic pain, the theory of re-occupying self. Seventeen individual interviews were recorded, with data collection, analysis and theory generation following classical grounded theory methodological approach. Constant comparison, theoretical sampling, theoretical coding, and theoretical sensitivity were used to identify the main concern of people who cope well with pain. This concern is achieving self-coherence, and is resolved by re-occupying self. Resolution involves making sense to develop an idiographic model of their pain; deciding to turn from patient to person, facilitated or hindered by interactions with clinicians and occupational drive; and flexibly persisting where occupational engaging and coping allow individuals to develop future plans. By completing this process, individuals form a coherent self-concept in which they re-occupy the important or valued aspects of themselves.
\end{abstract}

This study supports using Acceptance and Commitment Therapy because of its functional contextual view of people and their actions. This study illustrates that coping strategies are used in different ways depending on the primary goal within that context. Occupations, or active; purposeful; meaningful; contextualised and human activities, are used by people to make sense of their situation, and as a key motivation for developing coping strategies. 
These findings lead to new research questions about values-aligned activity, coping with identity change, and acceptance. 


\section{Acknowledgements}

Completing a Doctorate is a tribute to persistence. Obstacles during this one have included maintaining parenting and work commitments, coping with post-concussion syndrome, the tragic 2010-11 earthquakes in Canterbury, and in the final stages, moving into a caravan while our house was being repaired. Needless to say, that it has been completed is only due to the support, encouragement and occasional pointed comment from those around me.

Of particular mention are the efforts of my supervisors, Dr Jeff Gage and Dr Ray Kirk, who have been unfailingly kind in keeping me on track; my colleagues at both Burwood Pain Management Centre, and Department of Orthopaedic Surgery and Musculoskeletal Medicine at University of Otago in Christchurch; and my partner and family who have shown a remarkable tolerance for takeaway meals, a messy house and pain as appropriate subject matter for dinner conversation.

To my partner Bo, who has been the inspiration for asking how people who cope well with pain solve the problem of getting on with life - thank you for your patience.

To Andrew and Louise, who reminded me so often that I need to get on with it.

To Nick and Steve, who first helped me find my academic confidence.

To Arthritis New Zealand, and especially Suzanne Croft, who allowed me to ask for participants for this research.

To the participants, who inspired, refreshed and challenged me to understand flexibly persisting, and re-occupying self. 


\section{Table of Contents}

Living well with chronic pain: A classical grounded theory

Abstract

Acknowledgements

Table of Contents.

List of Figures. . X

Chapter One - Learning from those who live well with chronic pain.........................................................1

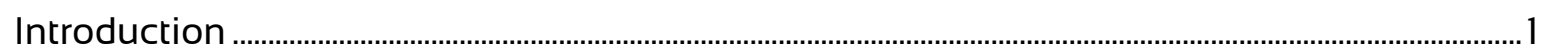

Reasons for learning from those who identify themselves as "well" despite pain.............3

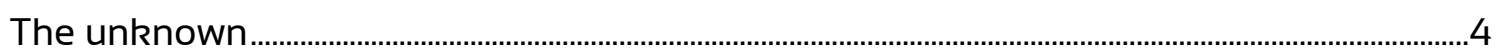

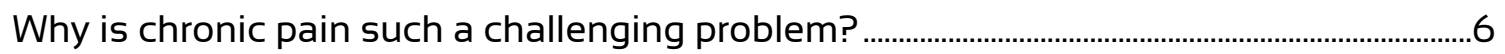

Why have people who are apparently living well with pain not been studied? ....................13

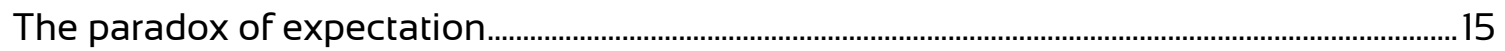

What can be assumed about people who manage pain well? .......................................................16

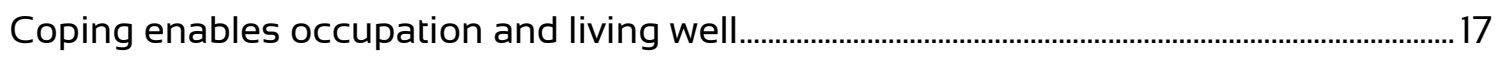

The need for a mid-range theoretical explanation for living well with chronic pain ......20

What is distinctive about a new theoretical explanation for coping well with chronic

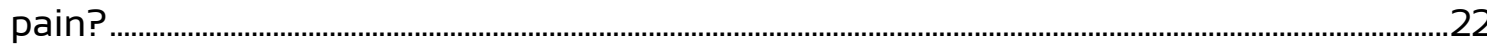

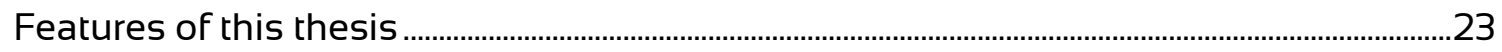

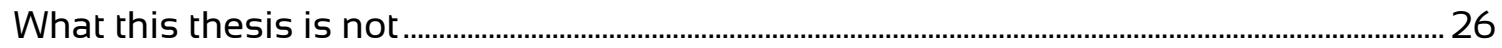

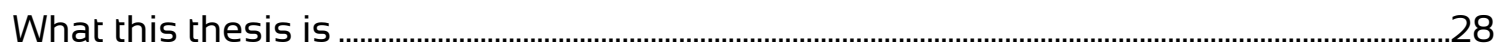

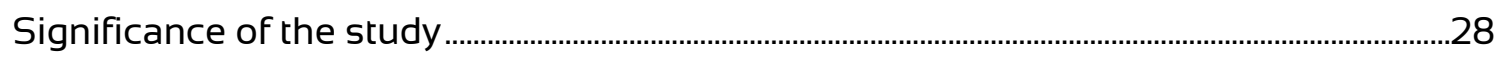

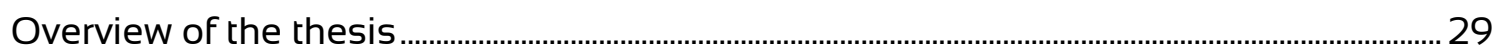

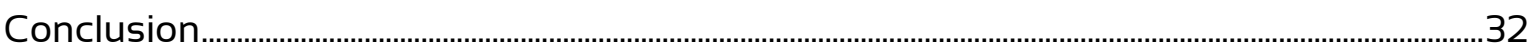

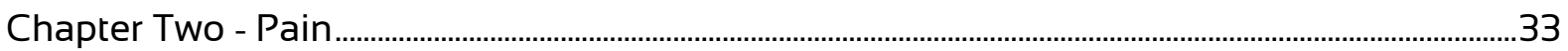

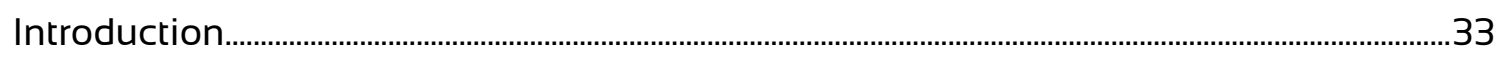

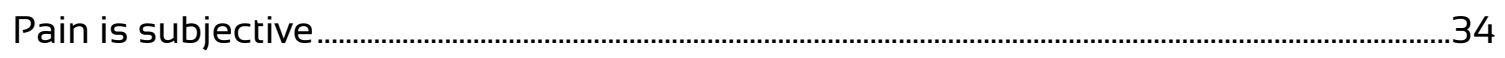

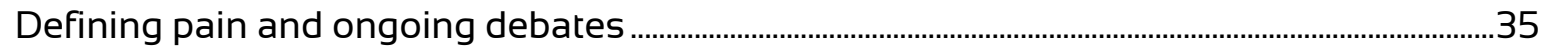

Distinguishing between pain and disability ……....................................................................... 41

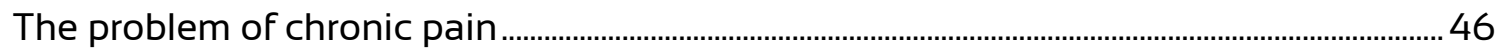




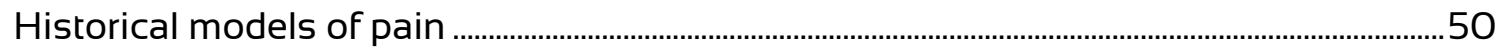

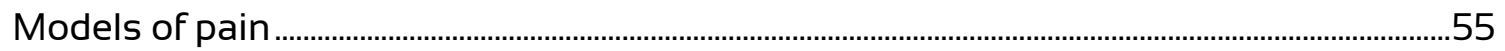

Introducing social context .......................................................................................................................... 64

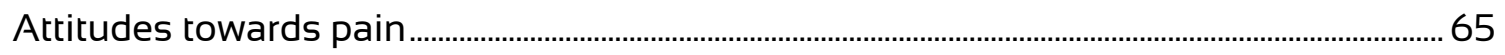

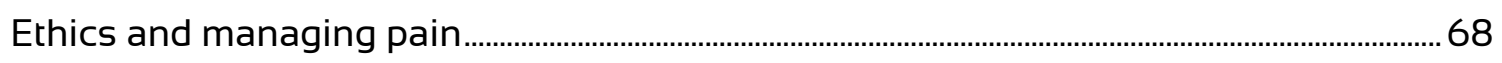

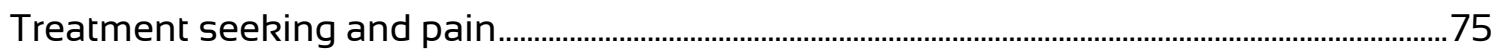

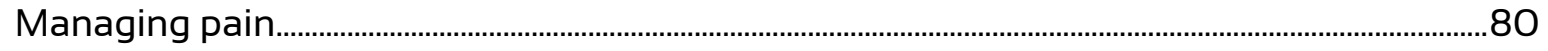

Coping

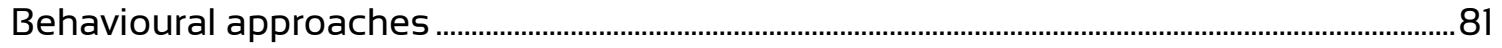

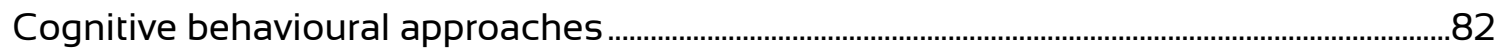

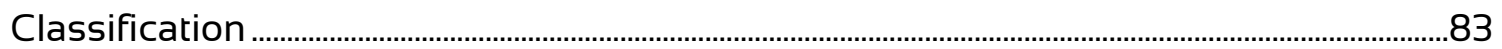

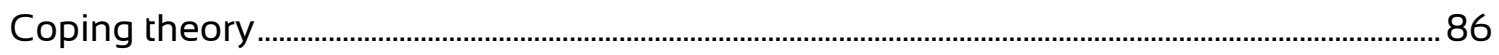

Strategies included in chronic pain management programmes ................................................90

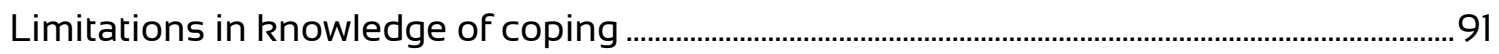

Moving towards models of ability and resilience in pain ................................................................. 93

Justifying a theory of how people positively manage chronic pain ...................................................99

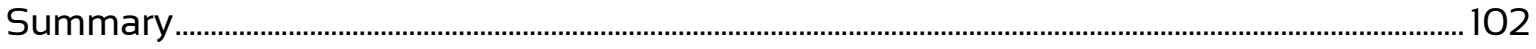

Chapter Three - Why use grounded theory? ................................................................................................ 103

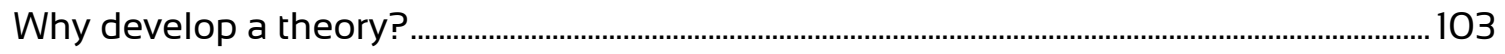

What is a theory?

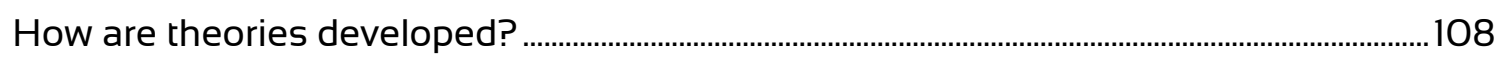

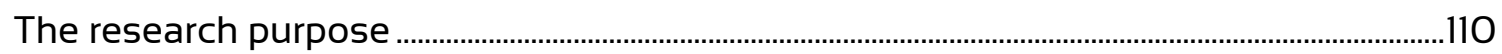

How this research question arose and how grounded theory may help answer it.........110

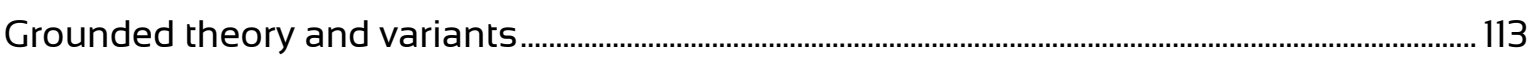

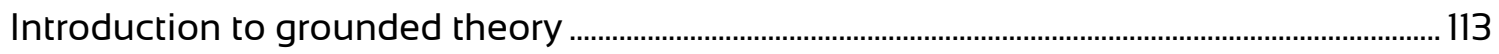

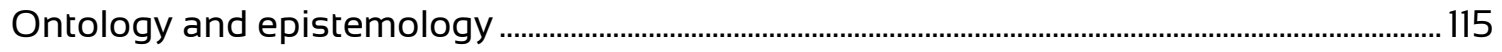

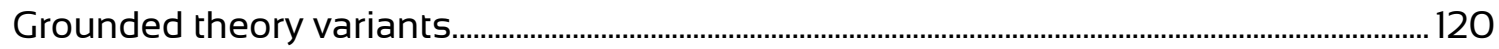

Grounded Theory and Symbolic Interactionism ................................................................................... 122

Dealing with pre-existing knowledge, and extant literature...........................................................124

Grounded theory and theory building

Meeting quality criteria for grounded theory studies .......................................................................... 131

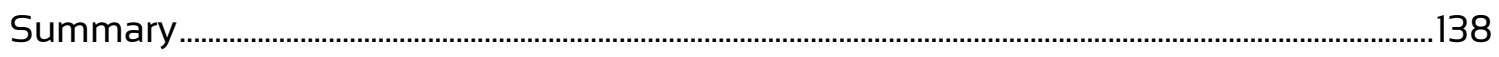

Chapter Four - Developing a classical grounded theory .......................................................................... 
Initiating research using grounded theory …………............................................................................. 141

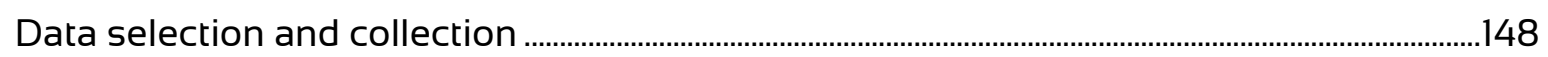

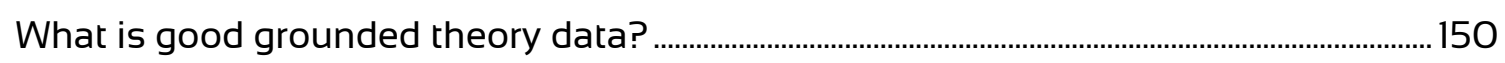

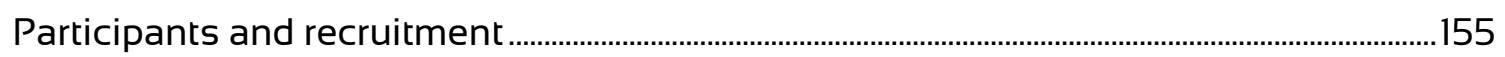

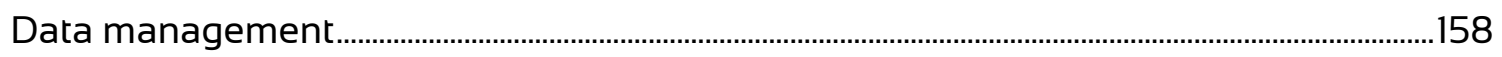

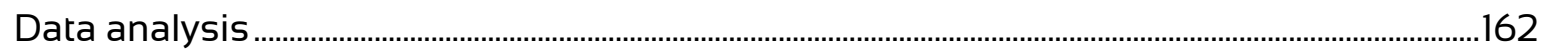

Developing a theory of living well with chronic pain .................................................................................167

Summary

Chapter Five - A model of living well with chronic pain ..............................................................................203

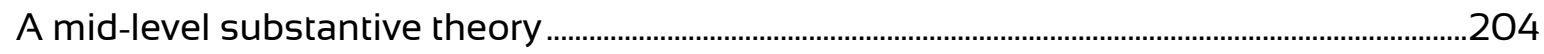

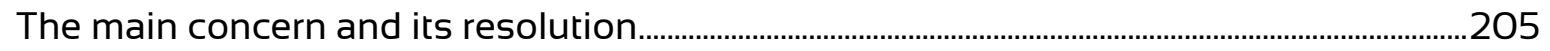

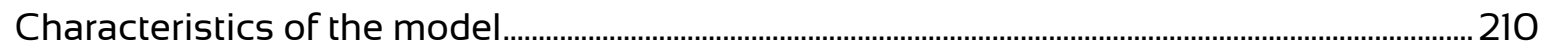

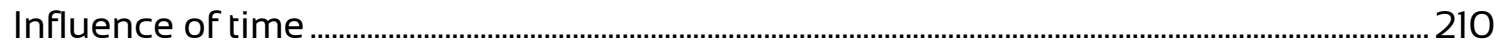

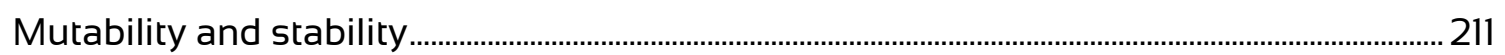

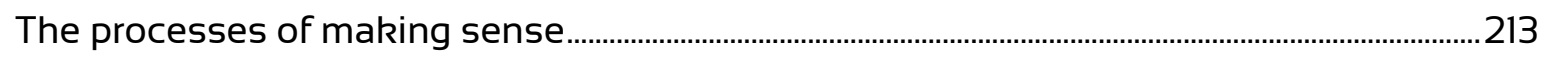

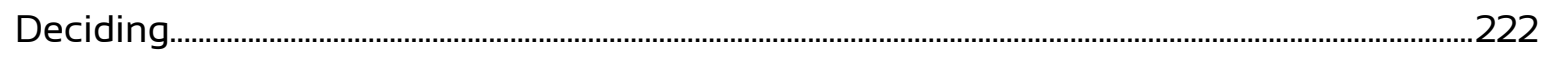

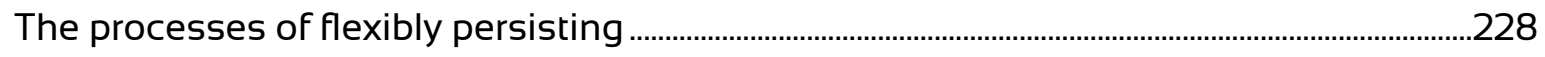

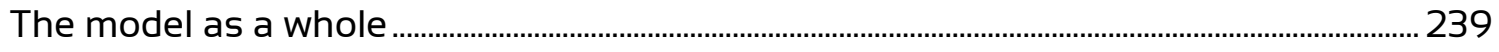

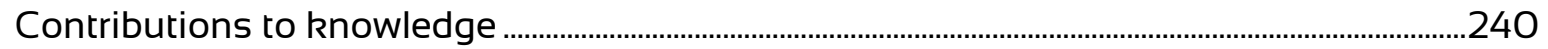

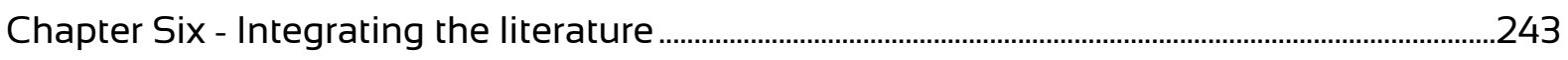

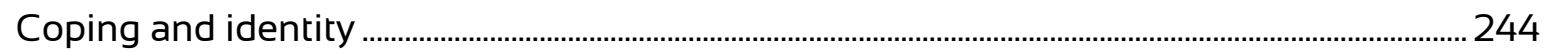

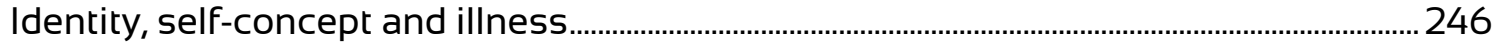

Self-discrepancy theory applied to those with chronic pain .............................................................. 249

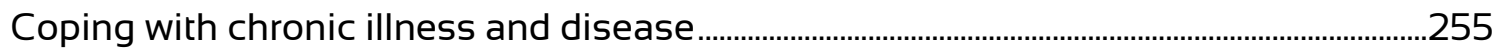

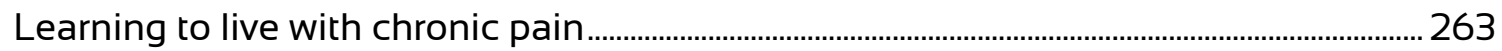

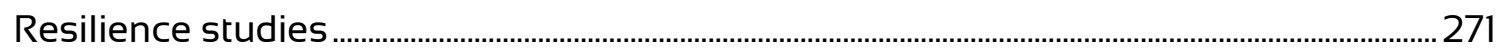

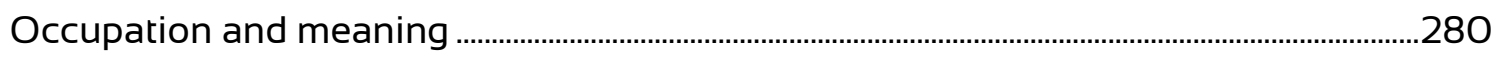

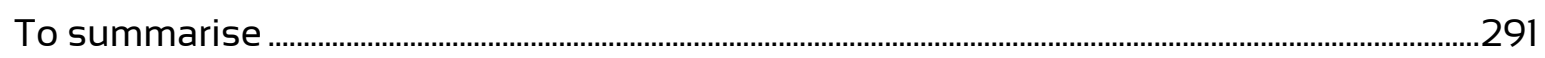

Chapter Seven - Contributions, Limitations, Opportunities and Conclusions............................ 294

Answering the research questions.......................................................................................................... 295

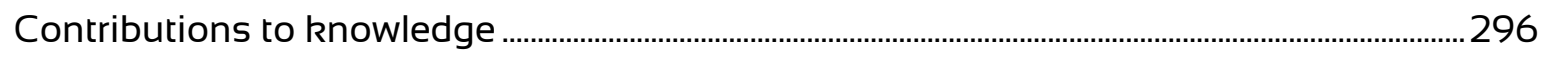

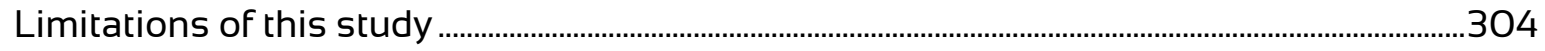




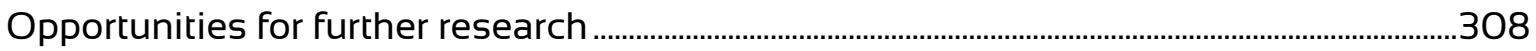

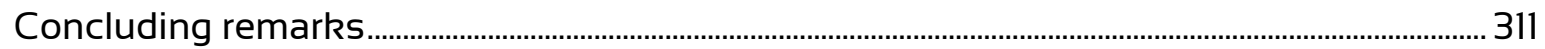

References

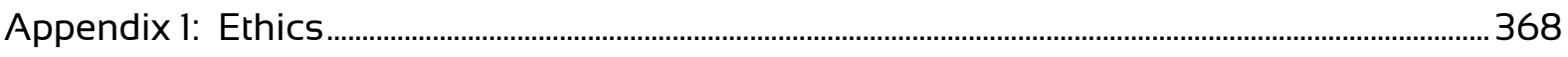

Amendments and Protocol Deviations ................................................................................................................369

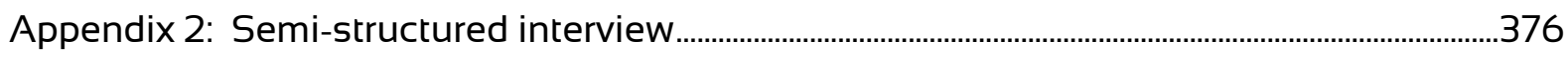

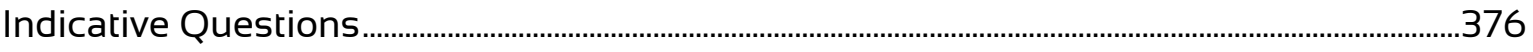

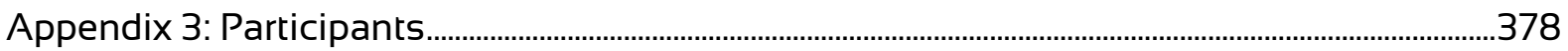




\section{List of Figures}

Figure 1. Biopsychosocial model (Engel, 1977). University of Rochester Medical Education. .9

Figure 2. Multi-faceted model of components of pain, Loeser (1982). .55

Figure 3. The "fear"-avoidance model developed by Vlaeyen and Linton (2000)... .60

Figure 4. Grounded theory process (Egan, 2002) 140

Figure 5. Example of NVivo analysis tool. 161

Figure 6. Excerpt from memo showing four codes (1) attitude to pain; (2) personal attributes; (3) it's a process, and (4) health professional did more. 169

Figure 7. Memo showing early thoughts about categories. 170

Figure 8. Memo showing coding integration 171

Figure 9. Memo recording properties associated with the category "getting on with it" ..... 172

Figure 10. Memo diagram illustrating relationships between three categories. 175

Figure 11. Memo illustrating properties of the category "getting on with life".. 176

Figure 12. Diagram drawn while attempting to fit categories into the "Six C's" basic psychological process.

Figure 13. Diagram illustrating "tipping point", then relabelled "Turning Point" and finally called "deciding".

Figure 14. Clarifying the relationships between categories, and raising the question of new pain..

Figure 15. Incidents coded to "doing what's important", later relabelled "Important Occupations".

Figure 16. (Overleaf)Memos considering category "doing what's important" as it evolved into "Important occupations"(a) "Doing what's important" memo (b) Table 2.1 from Molineux (2009) (c) Occupations and doing what's important

Figure 17. Indicators for category "Clinician who does more", later relabelled "Trustworthy clinician".

Figure 18. Memo illustrating relationship between category "Trustworthy clinician" and when participants decided to "get on with life". 196

Figure 19. Memo discussing Leventhal's model and my findings 199

Figure 20. Illustration of relationships between data and conceptual analysis 204 
Figure 21. Visual representation: Achieving self-coherence by re-occupying self.. 209

Figure 22. The process of making sense 214

Figure 23. The process of diagnostic clarity. 215

Figure 24. Process of occupational existing. 219

Figure 25. The process of deciding. .222

Figure 26. Engaging in occupations renegotiates self-identity. 231

Figure 27. Flexibly persisting. .234

Figure 28. Common Sense Model (Leventhal, Diefenbach \& Leventhal, 1992) .257

Figure 29. A biopsychosocial integrating framework for coping. Moos \& Holahan (2007). 


\title{
Chapter One - Learning from those who live well with chronic pain
}

\begin{abstract}
Introduction
"I have to keep going. I'm a mother. There's no-one else to do it."

"If I stop playing sport, I might as well give up. Sport is who I am."

"Well, I know what's important in my life and I just get on with it."
\end{abstract}

In a study examining attitudes towards treating people with chronic pain, "physicians noted high levels of frustration with their individual low back pain patients and in managing their care" (Allegretti, Borkan, Reis, \& Griffiths, 2010, p. 679), while in another study of acute low back pain management by general practitioners, the title of the paper began with "You feel so hopeless" (Breen, Austin, Campion-Smith, Carr, \& Mann, 2007). Chronic pain is mostly portrayed as a wholly negative experience for patients and clinicians alike. Chronic pain is pain that persists for longer than three or six months (Merskey \& Bogduk, 1994), and differs from acute pain in that the relationship between tissue damage and pain is no longer apparent, the pain has no functional benefit and psychosocial factors become increasingly important (Breivik, Collett, Ventafridda, Cohen, \& Gallacher, 2006; Main, Sullivan, \& Watson, 2008; Main, Foster, \& Buchbinder, 2010).

Some people, surprisingly, seem to deal well with chronic pain. These people report the same pain intensity as those who are seen in pain management centres, but instead of succumbing to distress and withdrawing from things they want or need to do, this "substantial minority (13\%)" (Karoly \& RuehIman, 2006) manage their pain effectively. 
In New Zealand, one in six people reported chronic pain in the $2006 / 2007$ New Zealand Health Survey. Two-thirds of the respondents indicated they had experienced chronic pain for five or more years, eroding their quality of life (Dominick, Blyth, \& Nicholas, 2011). Considerable research has been carried out over many years to identify characteristics of those who struggle with chronic pain, and to examine ways to help people live with less disability and distress. Researchers and clinicians have learned a great deal about the ways people manage chronic pain, and there are many theories to explain how disability associated with chronic pain in this population can be reduced.

People who remain well despite their chronic pain, however, seldom feature in the research literature. With some exceptions, research attention has been focused primarily on people who seek treatment, perhaps because this group poses the most problems for clinicians and the healthcare system as a whole; possibly because this group is very visible, or because clinical interventions address deficits and difficulties, rather than strengths and resilience. As a result, there is little theory to explain the ways people who seem unbothered by their pain, differ from those who have more trouble and scant information about how they approach managing pain in their daily lives. This results in limited knowledge about how they can be supported to strengthen or enhance their active approach to their pain. At the same time, the strengths and strategies used by these people remain invisible to the chronic pain management clinical and research community. This limits clinicians in the ways they can encourage people whom they see.

Many people with chronic pain do not respond to pharmacological or other procedures (Turk, Wilson, \& Cahana, 2011), and cognitive behaviourally-based pain management programmes show relatively small effect sizes (Williams, Eccleston, \& Morley, 2012). Researchers and clinicians are urged to develop better knowledge of therapy processes to help develop and refine interventions (Williams et al., 2012). Most research is, however based on people who seek treatment. Patients may respond to what clinicians offer during therapy but are only exposed to strategies that clinicians believe are useful. This 
may not represent the full range of strategies available. Clinicians may not know about the strategies used by people who do not seek help for their pain. Approaches offered in treatment may not be the strategies best used in daily life. It is time to learn from people living well despite pain.

Within this chapter, I establish the rationale for studying pain, and in particular, developing a theory to explain how those who describe themselves as living well, have reached this point. I briefly examine why chronic pain in particular is challenging to research, and why coping is an important topic. I justify the need for a theory to explain the process of living well with chronic pain. Essential characteristics of the classical grounded theory methodology structure how this thesis is written, and I point out features of the thesis to provide a framework for reading it. Learning from people who live well with chronic pain is a privilege, and I believe this thesis provides a unique insight into processes that may be useful for those who work with, research and perhaps live with chronic pain.

Reasons for learning from those who identify themselves as "well" despite pain

Seligman and Csikszentmihalyi (2000) first formalised the idea of establishing a scientific study of positive aspects of human experience, with the thrust of Positive Psychology being to understand and develop the strengths and qualities that enable individuals and communities to flourish.

To justify this new branch of psychology, Seligman and Csikszentmihalyi (2000) pointed out that psychologists had learned a great deal about how individuals "survive and endure under conditions of adversity", but relatively little of how normal people under normal conditions flourish ( p. 5). They proposed that psychologists had overlooked the opportunity to help people nurture and extend their positive qualities by focusing primarily on how people develop problems. Seligman and Csikszentmihalyi (2000) noted that most of the advances in preventing disease had come from perspectives aiming to build 
capability, rather than those modifying deficits (Seligman \& Csikszentmihalyi, 2000 p. 5). The need to prevent ill health precipitated the new focus - because while a disease or deficits approach to human behaviour can identify how problems begin or are maintained, it does not directly address how to prevent them from developing in the first place.

The benefits of adopting a focus on the positive are many, but one aspect that is particularly relevant to chronic pain management is the conviction that "merely relieving disease is not sufficient in creating well-being. The cultivation of well-being requires the presence of factors above and beyond those that alleviate distress ..."(Rusk \& Waters, 2013, p 218). Dunn and Dougherty (2005) note that having a disability does not negate an individual's other assets or resources (for example skills, proficiencies, personal qualities), and that by accentuating real or potential assets, the emphasis shifts from what people with disabilities cannot do to what they can do. Chronic pain management approaches, however, focus almost exclusively on clinicians helping individuals to challenge unhelpful thoughts, move from passive to active coping, modify activity patterns and develop new skills. Life, for a person completing a pain management programme, involves thinking about how to do everyday things in entirely new ways.

There is little known about the strengths that people with chronic pain bring to their situation; neither is a great degree of attention paid to how clinicians might identify, enhance, and encourage confidence in those strengths. There may be an untapped area of knowledge held by people who are not usually seen in pain management centres that researchers and clinicians could learn from, and transfer into their work.

\section{The unknown}

Chronic pain management programmes are costly (Loeser, 2006; Turk \& Okifuji, 2002; Turk et al., 2011) and the outcomes of programmes are moderate at best (Williams et al., 2012). Many participants in pain management programmes stop using the strategies introduced to them during such programmes, although they maintain functional gains 
(Curran, Williams, \& Potts, 2009), suggesting that something apart from the strategies themselves may promote change. This 'something' is as-yet unknown.

Determining which strategies should be included in pain management programmes, and the types of people these approaches may best help also continues to be an area of uncertainty. There are calls to use better research methodology, more careful participant selection, and improved clinician training to ensure that clinicians provide the best interventions. At the same time, some urge developing entirely new approaches to selfmanaging chronic pain (McCracken, Vowles, \& Gauntlett-Gilbert, 2007; McCracken \& Jones, 2012; Williams et al., 2012). Some of the emerging approaches place little emphasis on monitoring or controlling pain intensity and limited or no attention to challenging or restructuring thoughts and beliefs about pain. Greater importance is placed on identifying participant's values. By identifying personally-relevant values, individuals are more willing to engage and persist in activities, while mindfulness is used as a way to deal with painrelated distress (Lauwerier et al., 2012; McCracken, Gauntlett-Gilbert, \& Vowles, 2007; Vowles \& McCracken, 2008). Comparisons between traditional cognitive behavioural (CBT) approaches and acceptance and commitment therapy (ACT) show little difference in outcome (Veehof, Oskam, Schreurs, \& Bohlmeijer, 2011; Wetherell et al., 2011), and irrespective of the approach to managing pain, it is not yet known whether participants continue to use these strategies in the months and years following programme completion.

There is little known about how people with chronic pain use the strategies introduced in pain management programmes in their daily life, perhaps reflecting the challenges inherent in studying everyday life processes (Kikuchi et al., 2006; Smith, Brown, \& Ubel, 2008; Stone, Broderick, Shiffman, \& Schwartz, 2004). Technological advances mean it is substantially easier than 10 - 15 years ago to collect data as people go about their daily lives, yet there have been relatively few studies examining moment-by-moment coping in the chronic pain population (Sorbi et al., 2006a, 2006b; Stone, Broderick, Schneider, \& 
Schwartz, 2012). Despite the research undertaken to refine chronic pain management, many unanswered questions remain about what people do on an everyday basis while living with chronic pain.

Why is chronic pain such a challenging problem?

Chronic pain seems hard to understand despite intense research efforts by many researchers over many years. At least part of the difficulty is that managing chronic health problems is a relatively new phenomenon, emerging over the past $40-50$ years. Livneh and Martz (2007) suggest that this has occurred as people from industrialised societies live longer and develop age-related conditions such as eyesight and hearing loss, rheumatological conditions, cardiovascular and respiratory diseases; they note too that advances in biomedical technology mean individuals are more likely to survive trauma and disease (p. 3). Livneh and Martz (2007) also proposed that greater knowledge of the psychosocial consequences of surviving chronic health problems has led to greater interest in improving the quality of life for these individuals. Today's healthcare system must respond to more people with painful health issues, living for longer, and often expecting better healthcare and enhanced quality of life.

While these factors undoubtedly contribute to the rapidly expanding research into coping, possibly a greater reason for the difficulty understanding chronic pain is the legacy of historical models of pain.

\section{Historical legacies}

Pain has been conceptualised in many different ways over the course of human history. At various periods of history, people have viewed pain as the result of demon possession; punishment for sins; and the work of evil-doers casting spells (Bonica \& Loeser, 2001); through to modern conceptualisations such as psychiatric disorders (Engel, 1994), or nociceptor activation or neurobiological explanations (Waddell, 2004). Whatever the models of pain in the community, humans have attempted to understand and influence 
this universal experience, yet it remains a mystery in many ways. Indeed, there is still no 'gold standard' to determine whether someone is or is not experiencing pain (Sullivan, 2004).

Since Descartes (1596 - 1650), many individuals within the "Western" general public and healthcare community believe that there is a clear division between mind/soul and body. This has enabled the biomedical model to dominate thinking about health and illness. An extreme view of the biomedical model is predicated on the idea that all illness, signs and symptoms arise from a functional or structural abnormality of the body, and other factors such as the environment or personality are mostly unrelated to the onset, development or manifestation of the disease. Psychosocial concepts are irrelevant by comparison with the events within the body, and patients do not influence the onset or trajectory of the disease (Wade \& Halligan, 2004). The biomedical model has provided successful interventions to improve individual and community health through interventions such as hand-washing to remove bacteria and vaccinations against polio and smallpox; the model makes sense in many cases of illness or injury. A moderate version of the biomedical model still relegates psychosocial and contextual factors to a lesser place in human disease and illness experience.

Living within this context, when a person in a developed country experiences back, hip or knee pain it is likely he or she will think the problem must be caused by something that can be imaged with an X-ray or MRI (magnetic resonance imaging). In studies of lay or community beliefs about back pain, the biomechanical model dominates (Bowey-Morris, Davis, Purcell-Jones, \& Watson, 2011; Gross et al., 2006), and in individuals with low back pain, clinician's explanations are interpreted as confirmation that discs, vertebrae, muscles and nerves are responsible for the pain (Darlow et al., 2013). Surely by removing the bulging disc and preventing the vertebrae from moving by fixing it in place, the pain must also be eliminated? (Goubert, Crombez, \& De Bourdeaudhuij, 2004) This is not necessarily the case, as long-term outcome studies of back surgery have shown. For example, 
Bentsen, Rustoen, Wahl and Miaskowski (2008) surveyed patients who had spinal fusion between 1 and 8 years prior, and reported "low-to-moderate intensity" pain in neck, shoulders, back, hips, feet and legs. Hansson, Hansson and Malchau (2008) found that people with chronic low back pain who were given surgery improved very little, particularly in comparison with those receiving total hip joint replacements or total knee joint replacements (Hansson, Hansson, \& Malchau, 2008).

Interestingly Hanley et al. (2010) found that amongst orthopaedic surgeons attending a North American surgical conference in 2009 , only $23 \%$ believed that degeneration of the intervertebral disc is the primary cause of low back pain. Only one person of the more than 100 participants at the conference indicated they would undergo spinal fusion in the event of experiencing low back pain with degenerative changes at a single level (Hanley et al., 2010). In contrast, studies by Bowey-Morris et al. (2011); and Burnett et al. (2009) amongst many others, have found that beliefs about the biomechanical basis for back pain are highly prevalent both in healthcare students and the wider community. While some medical specialists might have moved from a purely biomedical model, many others and notably patients continue to hold inaccurate beliefs: the legacy of models from the past.

\section{New models of pain}

Over the past 30 years or more, there have been calls for what some have described as a "Kuhnian revolution" (Loeser, 2005a) or a paradigm shift away from the useful-butinadequate biomedical model to a more inclusive biopsychosocial model of pain and disability (Borkan et al., 2002). 


\section{HIERARCHY OF \\ NATURAL SYSTEMS}

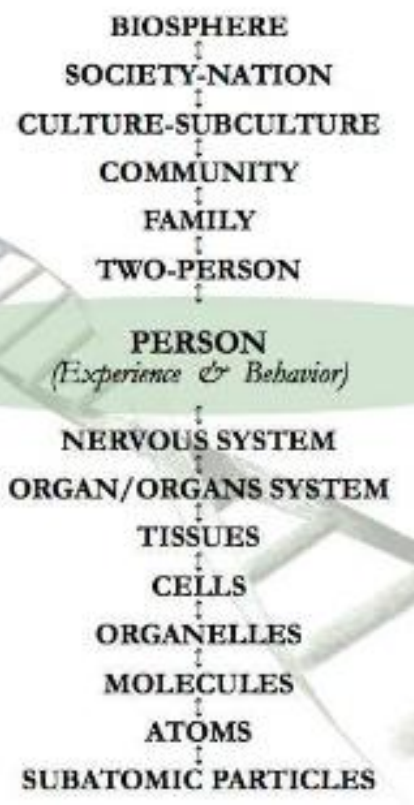

Figure 1. Biopsychosocial model (Engel, 1977). University of Rochester Medical Education.

This model, initially promulgated by Engel (1977), became increasingly accepted within pain research after Melzack and Wall proposed the Gate Control Theory (Melzack \& Wall, 1965). Their theory described nociceptive modulation by processes at the dorsal horn and higher up the central nervous system. The theory began to provide explanations for puzzling phenomena such as phantom pain and stress-induced analgesia and why, even after surgical excisions of supposed causes of pain, pain can persist and sometimes be worse (Melzack \& Wall, 1965). Melzack continues his writing today, and in an overview of pain highlighted that "the neuromatrix theory guides us away from the Cartesian concept of pain as a sensation produced by injury, inflammation, or other tissue pathology and toward the concept of pain as a multidimensional experience produced by multiple influences" (Melzack \& Katz, 2013, p. 1).

Researchers using the biopsychosocial framework have examined many aspects of pain that are not part of the biomedical model. Researchers using the cognitive-behavioural 
model, for example, have identified that problems with pain are not inevitably linked to pain intensity; instead, problems arise due to the way individuals appraise, or judge, their pain experience (Day, Thorn, \& Burns, 2012). Hadjistavropoulos et al. (2011) integrated aspects of communication, pain behaviour, attention and cognition in their biopsychosocial model of pain communication by drawing from anthropology, psychology, neurophysiology and sociology. Hadler and Carey (1998) describe what they call social iatrogenesis, or the adverse effects brought about when individuals with back pain seek care, initiating "a dynamic that predisposes to seeking care again" (Hadler \& Carey, 1998, p. 2) thus leading to greater periods of time on sick leave. These and other examples show that pain is much more complicated than a passive brain processing nociceptive input from the peripheral nervous system.

\section{What does the biopsychosocial model explain?}

The biopsychosocial model provides a framework through which a crucial distinction can be made between experiencing a pain and being disabled by it. IIIness behaviour occurs when an individual shows that they have pain via verbal or nonverbal behaviour. It is "observable and potentially measurable actions and conduct which express and communicate the individual's own perception of disturbed health" (Waddell, Pilowsky, \& Bond, 1989, p. 44). IIIness can be conceptualised as a social role, with certain privileges such as temporarily reduced social responsibilities (for example, lowered obligations to work, prepare food or travel); plus additional responsibilities such as striving to return to health, and seeking treatment to do so if necessary (Waddell et al., 1989, p. 42).

IIIness behaviour involves both voluntary and involuntary behaviour. Involuntary behaviour may include the withdrawal reflex, activating the sympathetic nervous system, limping or groaning, while voluntary behaviour ranges from simple behaviour such as rubbing or holding an area of the body through to complex behaviours such as applying for a sickness benefit or seeing a medical practitioner (Labus, Keefe, \& Jensen, 2003). Behaviours contribute the most to disability associated with chronic pain, rather than pain 
intensity; or more correctly, it is cognitions and emotions that lead to avoidance that are more disabling than the pain itself (Crombez, Vlaeyen, Heuts, \& Lysens, 1999).

Operant and respondent processes influence behaviours, invoking some of the social aspects of the biopsychosocial model (Fordyce, 1982b; Vlaeyen, De Jong, Onghena, Kerckhoffs-Hanssen, \& Kole-Snijders, 2002). Cognitive factors such as attention, beliefs, values, goals, and emotions also influence behaviour (Villemure \& Schweinhardt, 2010; Wiech, Ploner, \& Tracey, 2008). Much modern chronic pain management incorporates concepts from both cognitive and behavioural models of human behaviour to reduce illness behaviours while also enabling the individual to develop a future focus and sense of optimism (Patterson, 2005). The focus of treatment is not primarily on reducing pain; the aim instead is to reduce both distress and disability to enable the individual to have better quality of life (Main et al., 2008). The biopsychosocial model underpins many of the approaches used in cognitive behavioural pain management (Flor \& Turk, 2011) and although it has been nearly 50 years since the first cognitive behavioural pain management programmes were introduced, the approach is continually being refined.

The biopsychosocial model has helped investigators uncover processes that help develop understanding of how and why individuals with the same degree of tissue involvement may respond in different ways, and why the same person with a pain problem can respond differently in various contexts. The latest questions relevant to cognitive behavioural pain management are not whether the biopsychosocial model is useful for pain management, but about how the content or components of programmes might best be applied at the right time and to the right patients (Williams et al., 2012). By raising this question, however, many new questions arise. Chronic pain continues to pose problems for people with pain, clinicians and researchers.

\section{Questions about coping and pain management programmes}

Cognitive behavioural pain management programmes appear to obtain moderate treatment effects when participants' outcomes are compared with individuals on waiting 
lists. When these programmes are compared with active therapies, however, effect sizes are far smaller although remain clinically significant especially in respect to mood and catastrophising. They are, however, less helpful for disability and pain intensity (Williams et al., 2012).

In an appeal to improve the outcomes obtained by chronic pain management programmes, Williams et al. (2012) and others make strong calls for researchers to identify "which components of CBT work for which type of patient on which outcome/s, and to try to understand why" (Williams et al., 2012, p. 2). Commentators point out that cognitive behavioural approaches to chronic pain management remain a "black box" mix of various interventions, with limited knowledge of the processes of change involved, and no definitive theory to decide the format of treatment or who may benefit the most. Chronic pain management programmes can include many forms of intervention including such diverse approaches as hydrotherapy, mindfulness, activity management (pacing), and task simplification. The best combination or combinations of these methods needed for therapeutic change are not apparent, and there are be few limits on what can be included (Turk et al., 2011; Vlaeyen \& Morley, 2005; Vowles, McCracken, \& Eccleston, 2007b).

Current best practice from a biopsychosocial perspective reflects existing models that explain why some individuals struggle with their pain, with the focus on a relatively small number of psychosocial factors associated with high disability and distress. Treatment based on these models, therefore, looks to influence these factors; participants are supported to develop a selected range of strategies to help improve their quality of life and participation. Even the best treatments based on this approach, however, have limited success, suggesting there is considerable room to improve.

The following questions and more have not been answered yet:

- Which combination of interventions provide durable outcomes for people with chronic pain? Which interventions particularly address the needs of people who avoid moving because of their fear that pain will overwhelm them? 
- When should people be referred for pain management rather than focusing on pain reduction?

- How can strengths that individuals already use be incorporated into treatment?

- Who needs to have professional support? Which profession provides the most effective intervention? Does professional background matter?

- Why do some people manage well with their pain, how do they do this, and what can be learned from them?

While some of these questions have been answered in part such as approaches for people with elevated fear of pain (Vlaeyen \& Linton, 2012), and others are in the process of being investigated such as methods to support returning to work (Mehling et al., 2012; Sullivan, Adams, \& Ellis, 2012; Thompson, 2012), there are some that have not been extensively explored - including ways to incorporate individual strengths. The last question in the list above has been considered relatively seldom and is the subject of this thesis.

\section{Why have people who are apparently living well with pain not been studied?}

It is not true to say that this group have not been studied at all, but there are few studies in comparison with research into people who have more difficulty. Two studies conducted in New Zealand will be discussed in Chapter Six as the findings have direct relevance to my research questions (Large \& Strong, 1997; Strong \& Large, 1995). Theory development, to explain how and why some people manage well despite experiencing moderate to severe pain intensity, is similarly scant. It is not possible to know for certain why so little has been conducted, but some reasons may include:

1. The clinical models in chronic pain management are based on a deficits approach (Crowley-Matoka, Saha, Dobscha, \& Burgess, 2009). Cognitive behavioural approaches (CBT), for example, identify maladaptive coping strategies, unhelpful cognitive errors, and presenting problems. Functional restoration aims to correct postural problems, considers patients to be deconditioned, and looks for disability. 
If an individual reports using little analgesia and remains able to function, tertiary pain management services may not accept them because their disability is considered to be too low. The language indicates the need for individuals to change; that how they are currently managing are inadequate or ineffective.

2. Ways to reduce the cost to the health system and the general economy provide a strong rationale for providing chronic pain services (Goldberg \& McGee, 2011; Leadley, Armstrong, Lee, Allen, \& Kleijnen, 2012). Those, who do not seek treatment, use services or require pharmaceuticals, do not represent a cost to be reduced, and therefore may not feature when developing research priorities. Chronic pain has not been identified as a treatment priority in New Zealand, despite the large and growing number of people with health conditions known to be associated with chronic pain (Pfizer, 2012). If this group of high health users is not a priority, those people, who manage well, are likely to receive far less attention.

3. People who deal well with pain, may be difficult to find. Investigations into clinical pain (as opposed to pain elicited for experimental purposes) recruit participants from groups of individuals who are seeking treatment. Internationally, most researchers recruit from those seeking treatment at tertiary pain management centres, often associated with Universities. Additionally, chronic pain is invisible (Sim \& Madden, 2008); people with chronic pain come from all walks of life; there is a stigma associated with having chronic pain (Slade, Molloy, \& Keating, 2009), and there are many kinds of chronic pain, making it difficult to identify any one place where people with the problem might congregate.

4. This group of people may prefer to carry on with their daily life rather than identify particularly strongly with a 'chronic pain label', or they may simply be too busy to participate in research. 


\section{The paradox of expectation}

Human beings have always experienced pain, and human cultures have probably always sought and used ways to relieve pain. I explore the history of analgesia in more detail in Chapter Two, but at this juncture it is useful to recall that effective, relatively safe analgesia has only been available since the mid-1800s (Kulich \& Loeser, 2011). Until that time, experiencing pain from injury and disease was an expected part of daily life. Pain arising from degenerative disease, physical labour, childbirth and warfare was commonplace. Most medicinal herbs or procedures had adverse side-effects such as sedation or worse (blood-letting), thus taking them on a daily basis, would have posed problems (Warren, 1829). Withdrawing from activities or failing to work would not have been an option for many, especially in communities without state or church welfare. Individuals with persistent pain were expected to manage, and themselves expected to carry on because there were few alternatives (Bonica \& Loeser, 2001).

Once pain relief was widely available, however, and along with the successes of medicine in other aspects of health, expectations grew that pain should be abolished wherever possible (Loeser, 2005b). There is a call for pain management to be designated "a fundamental human right" (Brennan, Carr, \& Cousins, 2007). It is unsurprising that community attitudes in developed countries are, by and large, that pain should be abolished, and perhaps more importantly that it should be possible to abolish all pain. The situation is not clear-cut, however, as changing attitudes towards pain relief in childbirth demonstrate. From initial resistance and even hostility towards suggestions women could use analgesia in labour (Pearce, 2008), to the almost entirely anaesthetised state of "twilight sleep" in the 1950's and 1960's (Moscucci, 2003), and returning to today's natural childbirth sans pain relief or indeed any medical intervention (Lee Davis \& Walker, 2011), attitudes have shifted markedly. Obstetric analgesia is not an isolated example: people routinely participate in extreme sports involving significant physical demands; boxing and other contact sports often involve pain (Curry \& Strauss, 1994) while a small group of 
individuals deliberately seek pain as part of body modification or body suspension (Forsyth \& Simpson, 2008). I explore these contradictory attitudes in more detail in Chapter two when I discuss pain and coping.

Despite the presence of these paradoxical attitudes, the majority of research is carried out in communities where high expectations for adequate pain relief prevail. These expectations may not be met, particularly for those with chronic pain. Furthermore, clinicians cannot enhance what is offered to those who fail to respond to pain relief without developing a greater understanding of the approaches used by people who have pain but do not seek treatment.

\section{What can be assumed about people who manage pain well?}

It is extremely easy to find many studies identifying and examining the factors thought to contribute to distress and disability in people with chronic pain. It is far harder to locate studies establishing how those individuals manage when they remain in pain yet stop looking for treatment. There are many who continue to be disabled, distressed and demoralised (Baker, Gallois, Driedger, \& Santesso, 2011), yet disability undoubtedly occurs as a continuum from few limitations to profound limitations. Even individuals severely disabled by their pain incorporate a range of ways of coping into their daily lives, albeit often with short term benefits but unintended long-term consequences such as deactivation, depression and anxiety (Miles, Curran, Pearce, \& Allan, 2005; Snelgrove $\&$ Liossi, 2009).

From research identifying the characteristics of those seeking treatment for chronic pain it is reasonable to make some assumptions about those who are not actively seeking pain management:

- People in this group are coping with their pain well otherwise they would continue to be looking for treatment, although some people, who are not seeking treatment, 
may be unwilling or unable to seek treatment because of difficulty accessing services;

- They may have stoic personalities, or have some other personality characteristic that means they are not particularly bothered about their pain; or their stoicism could be influencing their help-seeking behaviour;

- Their pain may not be particularly intense, or it may be episodic or intermittent;

- They may not have the psychosocial risk factors for chronic disability, most especially they may not have mental health problems;

- They may use "active" coping strategies such as moderating their activity levels by pacing, regularly exercising and using relaxation techniques;

- They may not rely heavily on passive coping strategies such as resting, soliciting help or using medications on an as needed basis;

- They may have well-developed social networks for practical and emotional support;

- They may have a strong relationship/s with their treatment provider/s.

At this stage, there are no definitive data available to determine whether these assumptions are valid. Even if they are, they do not tell us much about how and why some individuals have learned to manage their pain and continue to function well. The process of learning how to handle pain while remaining active and undistressed, and more importantly why they do this without seeking further treatment is not yet understood.

\section{Coping enables occupation and living well}

Coping can be defined as "attempts to pursue valued activities and life goals" (Van Damme, Crombez, \& Eccleston, 2008, p. 2). Alternative definitions point to efforts to reduce psychological distress or to regain homeostasis (Livneh \& Martz, 2007, p. 10). The former definition incorporates a broader motivation for coping than that given by Livneh and Martz (2007), and supports the idea that coping does not occur in a vacuum; people employ coping strategies in order to do something, and people develop and alter the range 
of strategies they use depending on their circumstances. Within this thesis, coping refers to the process of pursuing valued activities and life goals despite the challenges posed when an individual experiences chronic pain. Coping strategies refer to the methods or ways used to continue pursuing valued activities and life goals despite the challenge of chronic pain. Living well represents a place on the continuum where coping strategies no longer dominate thinking or daily life planning.

\section{Occupation}

Occupational therapy and occupational science define "valued activities and life goals" as occupation. Although the precise definition continues to be debated, according to the World Federation of Occupational Therapists, occupation refers to "the everyday activities that people do as individuals, in families and with communities to occupy time and bring meaning and purpose to life. Occupations include things people need to, want to and are expected to do" (WFOT, 2010, para. 3). Occupations are imbued with individual and personal meaning, and differ from activity in that occupations are the unique and individualised way in which a person enacts a particular activity (Pierce, 2001). An activity, therefore, is a generic type of activity or role such as "cleaning teeth" while an occupation refers to the unique and individual way a person enacts "cleaning teeth" within his or her own environment, in the context of a daily routine, using a particular toothbrush and toothpaste. While one person may clean her teeth to keep them white and her breath smelling sweet, another person may do so to prevent tooth decay and reduce tooth sensitivity. Molineux (2009) submits that occupations have five characteristics: involving active engagement; being purposeful; meaningful; contextualised and human (p. 17), which suggests that irrespective of how occupation is defined, people are more able to do what is important in their lives by effectively using coping strategies.

When coping is reconceptualised as the process used to pursue what is important in life, the factors that influence the way individuals prioritise and enact valued occupations become as central to coping efforts as individuals' appraisals of their pain. Thinking of 
coping in this way involves evaluating the functions of various coping strategies in the context of an individual's life rather than thinking of them in isolation from why and how they use them.

\section{Living well}

Alongside the definitions of coping, coping strategies and occupation in this thesis is the notion of living well. People living with chronic pain have indicated that to them, and their families, coping can mean an intrinsic attitude towards life rather than the definition of coping used by health professionals (Barker, Reid, \& Lowe, 2014; Carroll, Rothe, \&

Ozegovic, 2013). Coping strategies and self-management have similar characteristics in chronic pain management, and some authors suggest there is a continuum in terms of knowledge, skill and confidence to self-manage, with those lower on this continuum construing coping as "compliance", while those at the higher end defining it as "being in control" (Dixon, Hibbard, \& Tusler, 2009; Morden, Jinks, \& Ong, 2011). The relative visibility of coping strategies versus living life may depend on how well-integrated the use of strategies has become. Living well, therefore, is at the highest end of the continuum, where individual coping strategies fade into the background, and life goals and occupations are most prominent.

Living well has also been a term used with reference to positive views of aging (von Humboldt, Leal, \& Pimenta, 2014; Zimmermann \& Grebe, 2014), living with diabetes (Eakin et al., 2013), effectively managing medical comorbidities (Friedman \& Ryff, 2012), and chronic ill health and disability (Gatchel, 2012; Goldberg et al., 2013; Mudge et al., 2013). The overarching theme drawn from these references is that individuals who live well no longer consider themselves as "sick" although still living with a disease; and using coping strategies to retain personal autonomy, remaining self-aware, and experiencing competence, relatedness and goal achievement (Ryan, Huta, \& Deci, 2013). Within this thesis, I have used the term living well to identify people who believe they are "in control", 
and for whom life goals and occupations are the primary focus rather than their chronic pain.

Coping and living well can be thought of as part of a spectrum of living with chronic disease. On the one end is not coping, or struggling, characterised by emotional distress and high levels of disability. At the other end is living well, characterised by little emotional distress, low levels of disability and little attention paid to how challenges posed by disease or illness are overcome. In the middle is coping, where attention to strategies needed to live life is required. Individuals, who are in the middle of this spectrum, are aware they are coping and identify as patients. Those who are living well, reject the label of patient, or disabled and consider themselves to be "people".

Researchers and clinicians need to know more about how people move towards living well despite chronic pain, in order to understand the mechanisms involved in effective chronic pain management. The variables of interest in relation to coping well may not be the converse of those associated with high levels of disability and distress. Effective coping may be related to avoiding strategies associated with greater disability, or with adopting strategies associated with less disability or both. The reasons for this group of people coping well could be something as yet not considered. There are few definitive answers at this time, and to avoid testing hypotheses that may lead nowhere there is merit in exploring and understanding more about those people who are naturally resilient.

The need for a mid-range theoretical explanation for living well with chronic pain

Mid-range theories bridge between the empirical level which consists of data and phenomena, and the philosophical level which is concerned with global perspectives and ways of viewing the world (Lierh, 2005). Mid-range theories generally refer to a particular factor or a substantive area, and help to explain and understand various structures and processes comprising the phenomenon of interest (Ward \& Hudson, 1998). When deciding 
the level at which to develop a theory, Ward and Hudson (1998) suggest researchers ask themselves the following questions:

- Where are the explanatory gaps, and

- What level of theory construction would be most helpful in advancing understanding at this point (Ward \& Hudson, 1998, p. 61)?

As I will show in subsequent chapters of this thesis, I contend that there is sufficient data to identify that a relatively large minority of individuals cope well with chronic pain. Existing theoretical explanations of coping explain why some people do not cope well, but do not explain much about those who are resilient. Theories or models of disability associated with chronic pain have mainly considered individual psychological factors such as catastrophising (Sullivan et al., 2006), pain anxiety (Larsen, Taylor, \& Asmundson, 1997), fear of pain (Crombez et al., 1999) and mood (Gureje, 2007). Earlier theoretical models also considered operant and classical conditioning as potent moderators of pain behaviour and disability (Fordyce, 1974). Intrapersonal factors such as the kinds of coping strategy employed (problem-focused or emotion-focused) have been examined (Leventhal, Diefenbach, \& Leventhal, 1992), but although situational or contextual factors such as the severity and duration of stressors have been considered in some settings (Livneh \& Martz, 2007), these have rarely featured in models of chronic pain coping.

Studies examining resilience have identified a two-factor model of affective health in people with chronic pain thought to be "resilient" (Smith \& Zautra, 2008). This model has lately been extended to integrate both intrapersonal and social factors to explain how some individuals recover, sustain their recovery and find meaning from the experience. It provides an interesting approach to conceptualising both modifiable and stable factors that may influence resilient functioning (Yeung, Arewasikporn, \& Zautra, 2012). It does not, however, explain the decision-making process used by ndividuals to decide when they will "recover", to use the definitions coined by Sturgeon and Zautra (2010), and while social relationships are included, these refer to broad social engagement by the individual. 
Health provider and community attitude influences are not considered. Furthermore, the motivation for coping is not explored. This means that some of the most persuasive relationships relevant to the way an individual understands their diagnosis are not currently included, and reasons for wanting to cope are not integrated into the model. I believe that finding a way to incorporate these latter factors within theories of coping with chronic pain must begin by learning directly from those who believe they are coping well. These individuals likely show some consistencies with one another in terms of how they view their pain; how they have negotiated the process of deciding to recover (Sturgeon \& Zautra, 2010) or bounce back (Smith, Dalen, et al., 2008); what they do to cope and maintain their involvement in important activities, and finally, there should be some commonality in terms of their overall motivation to 'keep going' despite their pain.

\section{What is distinctive about a new theoretical explanation for coping well with}

\section{chronic pain?}

This thesis details an integrated theoretical explanation for how and why some people cope well with chronic pain. Using classical grounded theory, the study identifies the processes involved in moving from recognising initial symptoms through to maintaining wellbeing despite pain. The model details the salient characteristics of this population; indicates crucial aspects of their interactions with health professionals; identifies factors that influence various decision points, and details the ways they have maintained their positive progress.

The model or theory (I will use the terms interchangeably) is consistent with much of the existing literature, but differs from current theory because it integrates a number of existing theoretical propositions into an explanatory model within a population that has not often been studied. It is also a model that draws directly from empirical data provided by participants themselves rather than theories or conjecture; thus it has validity within the substantive area. 
By developing an understanding of how and why people who cope well have reached this place, clinicians working with individuals who have difficulty managing their pain may be able to enhance coping rather than mitigate problems. Information obtained directly from individuals who cope well may more likely represent the daily reality of living with chronic pain and provide useful clues for chronic pain management programme development. If living well is viewed as a dynamic and evolutionary process that occurs within the social context of daily life occupations, the focus of pain management for those seeking help may need to shift towards factors supporting people to develop a future focus and maintain positive emotions.

By using grounded theory, there is an empirical basis to support the hypothesised interactions between important and consistent variables that have emerged from the data. Further testing will verify and elaborate on these relationships. Generating hypotheses from a model grounded in data differs from the traditional hypothetico-deductive approach where developing a hypothesis is rarely discussed, and most attention is paid to testing competing hypotheses. By using empirical data, and through abductive reasoning, or reasoning back from data to a new explanation (Josephson \& Josephson, 1996), hypotheses generated using classical grounded theory can provide a meaningful platform for understanding the processes involved in living well with chronic pain.

\section{Features of this thesis}

I have used classical (Glaserian) grounded theory to explore how and why a substantial minority of people with chronic pain remain resilient; that is, although they experience moderate to severe pain, they remain undistressed and not disabled. Grounded theory methodology possesses some characteristics that require explanation at the outset of this thesis, because these features influence the structure of writing. 


\section{Literature review}

Classical grounded theory is an inductive approach to discovering latent variables from data obtained directly from the substantive area of research. In this approach, the researcher is the research 'instrument'. Different steps and conventions apply compared with many other methodologies. The first and most obvious difference is the place and purpose of the extant literature.

Most research theses involve the researcher presenting a literature review, identifying gaps in the literature, seeking to fill those gaps by developing a hypothesis, presenting the findings, discussing the implications, and drawing some conclusions. Researchers using classical grounded theory do not always follow this approach.

Glaser contends that comprehensive knowledge of the literature in an area can force the emerging theory to fit with what researchers already know, leading to inaccurate assumptions about what should be found as opposed to what is present (Glaser, 1998). The extant literature is certainly not ignored, but researchers must first identify what exists in the data obtained from the substantive area, and then weave relevant literature into the emerging theory only where the data justifies its inclusion. Existing research is used to develop the burgeoning theory, to nest the new theory in current discussions, to develop arguments and optionally to comment on the literature in relation to the method.

Consequently I do not provide a separate literature review chapter in this thesis; instead I explore the background to the problem of chronic pain and discuss broad theories of coping before turning to questions of appropriate methodology for examining the research question.

\section{Personal perspective}

Using a human as the research instrument rather than carefully calibrated instruments or psychometric measures can pose a problem with bias. I have worked as a clinician and educator in the field of chronic pain management for many years and have developed 
views about chronic pain and its management on this basis. Implicit biases cannot be readily identified nor mitigated (Greenwald \& Krieger, 2006); thus, the concept of a valuefree and objective study usually assumed in positivist or realist research cannot be easily achieved, if indeed it is possible in any piece of research. Glaser (2007) argues that classical grounded theory is not yoked to any particular philosophy of science, and he also rejects the constructivist view that meaning is co-created between researcher and participants. Irrespective of any personal opinion regarding Glaser's philosophy of science or co-creation of meaning, Glaser's approach provides a useful way of dealing with the problem of pre-existing knowledge: the researcher conducts an interview with him or herself. He points out that any views expressed by the researcher need also to be found elsewhere before being incorporated in the emerging theory (Glaser, 1998). In Chapter Four, therefore, I present my personal perspectives on philosophy of science, chronic pain, and coping, to acknowledge the degree to which my thoughts and opinions may have influenced the resultant theory.

In addition to describing the beliefs and attitudes I bring to this research, I also write in first person throughout the thesis to acknowledge my active role throughout the research process, including writing the analysis. Writing this way helps to make reading the thesis easier, and to maintain a consistent, active voice throughout the document..

\section{Theory generating rather than theory verifying through hypothesis testing}

This thesis presents a substantive theory. Substantive theory applies to a specified area of concern, as distinct from formal theory which applies to a general area of, for example, coping or deviance (Glaser \& Strauss, 1965). Classical grounded theory does not test the hypothesised relationships between the variables (usually called categories in classical grounded theory, although Glaser uses the terms interchangeably) because it is grounded directly in empirical data (Nathaniel, 2011). This means the predictive power of the theory is limited to the substantive area of those people who cope well with chronic pain. Because it is generated from real (grounded) data, it is by nature already verified within the 
substantive area (Glaser \& Strauss, 1965). The primary purpose of classical grounded theory is to generate new theory, rather than to verify or test theory.

Part of my rationale for choosing classical grounded theory is also because it can accommodate a realist philosophy of science, and the model can be further developed and generalised to other populations or phenomena through hypothesis testing, structural equation modelling (Rosenbaum, 2011) or by generating a formal grounded theory (Andrews, 2012). These steps are conducted after the substantive theory has been developed (Glaser \& Strauss, 1967). Consequently in Chapter Seven I suggest avenues for future research to detail the relationships between categories, and to extend the theory to different populations.

\section{What this thesis is not}

Before I describe the structure of this thesis, I want to identify what I do not aim to do. I aim to generate a substantive theory to explain how people cope with chronic pain, developing this from the perspective of those who believe they are living well.

1. The theory is not intended to explain why or how people do not cope well, there is already much written in this regard. Although it is tempting to consider how the findings from this study could explain why some people have trouble coping with their pain, the data used are drawn from individuals who do cope well, thus there is no empirical basis for drawing such conclusions. Aspects of the theory could, however, be used to alter the trajectory of people who are at risk of poor coping.

2. The thesis does not represent the final word on how people who cope well with their pain do so: Glaser points out that good grounded theory should be capable of being modified (Glaser, 1998), and acknowledges that a grounded theory does not encompass every piece of data available in the substantive area. Instead, classical grounded theorists are encouraged to identify participants' main concern, discover the core category that resolves the main concern and delimit to only those 
categories associated with the core category (Glaser \& Strauss, 1967). It is probable the categories identified in this study could be extended or detailed through other variables or categories in future research.

3. This grounded theory is concerned with how people who cope well with their chronic pain have remained actively involved in their daily life. There are aspects of the way these individuals have coped that have not emerged as relevant to this grounded theory such as how they have managed interpersonal relationships: these remain areas for future research into the lives of people who live well despite their chronic pain.

4. In order to avoid confounding variables well-known to influence coping and disability, individuals receiving weekly compensation from New Zealand's national accident insurer, Accident Compensation Corporation, and also those with other chronic conditions such as chronic obstructive pulmonary disease or multiple sclerosis were not included in the study.

5. Finally, the thesis does not examine the neurobiology of pain in any detail. There are several reasons for this, not the least of which is the rapid growth of knowledge about these aspects over the past ten years (Edwards, Campbell, Jamison, \& Wiech, 2009). In addition, there is ample information suggesting that irrespective of the mechanisms of pain, the most relevant factors influencing the way individuals manage pain are the views individuals have on their pain (Anderson \& Hanrahan, 2008; Chalk, 2007; Ramirez-Maestre, Esteve, \& Lopez, 2008; Wiech et al., 2008). Even though there is evidence to suggest that some forms of coping practice alter neural pathways involved in pain (Edwards et al., 2009), it is unlikely individuals with pain would report or describe their practice in these terms, thus making it impossible to identify via interview. 


\section{What this thesis is}

Within this thesis I develop a theoretical explanation for how and why some people cope well with chronic pain to extend the understanding of how people use pain-related coping strategies in daily life, and understand some of the factors associated with a successful chronic pain trajectory.

I contend that researchers and clinicians can learn a great deal about the process of learning to live well with chronic pain, and that this can inform daily clinical practice. It can also raise new explanations, and hypotheses for future study as a result of deriving information directly from, or grounded in, the data. As a consequence of this work, I hope clinicians will have a more comprehensive understanding of how they can support people with pain to develop resilience, and remain engaged in occupations and lifestyles of their choice.

My research questions are:

- How do people who think of themselves as well despite having chronic pain carry out their important daily tasks?

- How did they develop these strategies?

- What are the factors influencing how and why they live well despite their pain? In grounded theory terms, my research questions are:

- What is the main concern of people who identify themselves as living well with chronic pain?

- What is the process followed by this group of people?

- How do these people achieve their important goals despite persistent pain?

\section{Significance of the study}

I believe the research in this thesis is valuable for a number of theoretical and practical reasons. As indicated above, most research into chronic pain coping focuses on people 
who seek treatment for their problem. North American and European Union-based research dominates the literature, and participants are mainly those attending large tertiary pain services. Conclusions drawn from this body of research may not apply to New Zealand residents who cope well with their pain. A mid-range theory, developed from, or grounded in, the experience of people who cope effectively with chronic pain, can help integrate this research and make it more useful in the New Zealand context. It may also provide a basis for determining how closely research conducted elsewhere in the world might apply to people living in New Zealand. Furthermore, findings from this group of people may inform clinicians and researchers interested in understanding and supporting resilience in those who have more difficulty living with their pain.

The grounded theory of living well with pain explains how people move through the process of coping with pain, from their initial response to successfully living with their experience. It also illustrates how and why they employ a range of coping strategies. Importantly, it demonstrates the ways people integrate strategies into daily life. This is particularly valuable for ensuring a patient focus during goal-setting, and for enhancing the utility of pain management programmes to meet the long-term needs of participants. The theory offers a functional contextual interpretation of coping, going beyond viewing coping strategies in isolation from the context and purpose in which they are used, and considering the purposes for which strategies are employed.

Finally, the model proposed as part of this thesis provides an integrated heuristic model for researchers wanting to understand the process of adjusting to chronic pain. The participants in this study did not always "live well" with their pain. The model provides opportunities to develop interventions to enhance positive coping; these can be used to alter the trajectory of people less able to cope successfully with their pain.

\section{Overview of the thesis}

The thesis is structured in eight parts: 


\section{Chapter one: Introduction}

In this chapter, I have introduced my thesis by describing the context and purpose of the study. I also justify the thesis in terms of its theoretical and practical importance.

\section{Chapter two: Pain}

The focus of Chapter Two is on pain and chronic pain in particular. By providing a brief history of pain models, I discuss sociocultural influences on attitudes towards pain, treatment seeking and pain relief, and use the example of attitudes towards pain relief in childbirth to illustrate how quickly attitudes can change towards pain. While childbirth represents acute pain, attitudes about the use of analgesia reflect prevailing societal views about pain more generally, including views about chronic pain and its management. I review the interaction between healthcare providers' attitudes and community attitudes, taking particular notice of the ways in which treatment seeking may be influenced by individual attitudes, as well as community and treatment provider beliefs. Finally, I briefly review concepts of coping, in particular the need to explore explanations that go beyond conceptualising coping as separate from the context in which they are used, and the value of examining efforts that go beyond just remaining in status quo to incorporate concepts of resilience and flourishing.

\section{Chapter Three: Why use grounded theory?}

The next chapter details the methodology I chose. I move from a macro to micro view, reviewing why a theory is justified, how theory is developed, and then the processes used in generating my grounded theory.

I begin in Chapter Three by identifying the purpose for developing theory, defining theory and discussing how theory is developed. I justify the need to develop a theory then show how grounded theory is appropriate for answering my research questions. I distinguish between the three main forms of grounded theory by pointing out philosophical differences, as well as procedural ones. I specifically discuss two aspects of grounded 
theory that can be misconstrued: symbolic interactionism, and dealing with pre-existing knowledge and the extant literature. I finish this chapter by discussing the quality criteria used to evaluate classical grounded theory.

\section{Chapter four: Developing a classical grounded theory}

I then move to Chapter Four where I use the research process outlined by Egan (2002) to describe the practical aspects of developing a grounded theory. I begin by discussing various influences on my choice of the research question and methodology and detail my beliefs as they affect this study, before considering relevant ethical issues.

Within this chapter, I present my approach to data selection, and practical considerations when managing data in a study of this nature. Interviewing forms a large part of the approach to data collection, and I discuss the approach required when using interviewing for research. I also discuss how I recruited participants to the study in light of both the type of study, and the tumultuous events of the Canterbury earthquakes in 2011.

The final sections of this chapter describe and demonstrate the data analytic techniques I used in this study. This section of the chapter is structured to present how I implemented classical grounded theory and rigorously followed its requirements throughout this study.

\section{Chapter Five: A model of living well with chronic pain}

In Chapter Five, I present the theoretical model Living Well with Chronic Pain, along with the extant literature relating to the findings from my study.

\section{Chapter Six: Integrating the literature}

I then discuss implications of my findings in terms of theoretical and clinical importance.

\section{Chapter Seven: Contributions, limitations, opportunities and conclusions}

In the final chapter of this thesis, I summarise and integrate the material discussed throughout the thesis, and present the main contribution to knowledge drawn from the 
findings. I consider the limitations of this work, and finally conclude with areas for future research.

\section{Conclusion}

Chronic pain is a burden for many people, clinicians and patients alike. By developing ways of helping people manage the constraints of chronic pain the human and economic cost of this problem may be reduced. Learning from people who live well provides insights into how life can be lived despite pain and offers opportunities for clinicians to improve the lives of those who find it challenging.

In the next chapter (Chapter Two), I discuss pain and why chronic pain presents such a challenge. I discuss the way theory influences clinical practice and the problems that can arise when clinicians make assumptions about what needs to be addressed therapeutically after reviewing historical models of pain,. I argue for moving beyond a deficits model of coping towards models of resilience and ability as I justify studying those individuals who cope well with their pain. 


\section{Chapter Two - Pain}

IT would be a great thing to understand Pain in all its meanings. To understand it, first of all, in its largest or universal meaning; what it is in its own nature; how allied to the various morbid states and conditions in which it is found, how formed by them, how evolved from them. (Latham, 1862, p.677)

\section{Introduction}

Pain - collectively, all humans have experienced it, yet while there have been rapid advances in understanding neurobiological mechanisms associated with pain, the feeling of what it is like to have pain (qualia) continues to be personal, private and solitary. In this chapter, I begin by defining pain and explore aspects of the current understanding of pain. I distinguish between acute pain and chronic pain. Within this thesis and my research study, I have defined chronic pain as being the presence of pain more days than not, over a period of three months or more, in line with Dominick et al. (2011). This enables comparison between my study and other New Zealand-based research. I will, however, discuss the utility of a definition tied to pain duration.

In discussing pain, I examine why studying pain, and particularly chronic pain has been so complicated. The history of pain and pain management provides useful examples to illustrate how fluidly beliefs and practices change over time. I discuss the Implications arising from the international research and clinical "paradigm shift" towards a biopsychosocial model of pain and its relevance to understanding and managing pain. In doing so, I discuss attitudes towards pain and treatment-seeking. I then turn to coping as a construct, and examine the way this has been construed in chronic pain management, and in particular how this reflects societal beliefs about health, disease, pain and disability. 
I trace the history of psychologically-based chronic pain management because it is from research within this tradition that coping has been construed. I also note the limited attention paid to how individuals use coping strategies in daily life, particularly in the context of coping well. I conclude by justifying the value of studying those who cope well with pain.

Pain is a human experience and attitudes towards pain by those in the midst of the experience, those who would help them, and those who are within the context of the person with pain, demonstrate fluid and flexible attitudes towards this most human problem.

\section{Pain is subjective}

Pain is difficult to study. It is a subjective state: the experience of pain cannot be directly shared. It is common to all humans, and individually we are familiar with at least our own experience. This familiarity can be both helpful and unhelpful. One's own experience of pain engenders assumptions about the ways other people experience and express pain. Simple measures of pain give some insight into another's pain, but fail to capture the meaning of pain as it is experienced by an individual.

The only way to determine whether another person is feeling pain is through the individual's behaviour (Hadjistavropoulos et al., 2011). Measurements provide a common language through which individuals can convey at least some aspects of pain. The unshared assumptions, however, of the "intrapersonal and interpersonal or contextual factors that might influence the extent to which a sufferer's pain becomes manifest socially and, hence, is expressed behaviourally (Hadjistavropoulos et al., 2011, p. 3)" provide much room for misunderstanding an individual's internal experience, and in particular, the unique meaning of pain in the context of a person's life. 
Assuming that all individuals share the same pain experience as one another can lead to believing that all individuals share the same amount of suffering, and therefore need the same relief from suffering. These assumptions subsequently influence clinical practice. I will show that these assumptions may be incorrect in a later section of this chapter, but before I do, I discuss the International Association for the Study of Pain definition of pain.

\section{Defining pain and ongoing debates}

\section{International Association for the Study of Pain (IASP) Definition}

The current internationally accepted definition of pain is "Pain: An unpleasant sensory and emotional experience associated with actual or potential tissue damage, or described in terms of such damage." (International Association for the Study of Pain, 2005).

The definition is noteworthy for focusing on pain as an experience associated with actual or potential tissue damage, rather than assuming it must inevitably be associated with tissue stress or damage. The following note extends and supports the definition, reproduced in full below:

The inability to communicate verbally does not negate the possibility that an individual is experiencing pain and is in need of appropriate pain-relieving treatment. Pain is always subjective. Every individual learns the application of the word through experiences related to injury in early life. Biologists recognize that those stimuli which cause pain are liable to damage tissue. Accordingly, pain is that experience we associate with actual or potential tissue damage. It is unquestionably a sensation in a part or parts of the body, but it is also always unpleasant and therefore also an emotional experience. Experiences which resemble pain but are not unpleasant, e.g., pricking, should not be called pain. Unpleasant abnormal experiences (dysaesthesias) may also be pain but are not necessarily so because, subjectively, they may not have the usual sensory qualities 
of pain. Many people report pain in the absence of tissue damage or any likely pathophysiological cause; usually this happens for psychological reasons. There is usually no way to distinguish their experience from that due to tissue damage if we take the subjective report. If they regard their experience as pain, and if they report it in the same ways as pain caused by tissue damage, it should be accepted as pain. This definition avoids tying pain to the stimulus. Activity induced in the nociceptor and nociceptive pathways by a noxious stimulus is not pain, which is always a psychological state, even though we may well appreciate that pain most often has a proximate physical cause (2014, par 4-5).

There are several historically significant points within these explanatory notes. The first is that pain is subjective. Subjectivity contrasts with objectivity: being objective means not influenced by personal feelings or opinions, existing independently of the individual, real or unbiased while being subjective implies a personal, individual, unique and internal experience, one that cannot be judged as biased, or indeed, unbiased (Hegelund, 2005; Moskovitz, 2011; Phillips, 2007). A common misconception exists that without evidence of tissue damage, there can be no pain, or that the pain is "psychogenic" (Covington, 2000). The IASP definition prioritises the legitimacy of an individual's experience over any external evidence by placing pain within the domain of subjectivity. The authors reinforce this point in the later part of the notes, where they state that if an individual reports that they have pain "as if" tissue damage caused it, this should be accepted, although a person may do so for "psychological reasons". This latter point is arguable: in recent years functional magnetic resonance imaging (FMRI) studies have demonstrated subtle changes to the neurobiological substrate underpinning pain, suggesting that so-called "psychological" processes may instead be neurobiological (Latremoliere \& Woolf, 2009; Ploner, Lee, Wiech, Bingel, \& Tracey, 2011; Tracey \& Bushnell, 2009; Woolf, 2010). A further implication of subjectivity incorporated within the definition of pain is that it is, therefore, 
impossible to determine whether an individual is "faking" or feigning their pain. In other words, if a person says they have pain, this is considered true.

The second point is that individuals learn about pain and its association with injury as part of normal development; implicit within this point is the notion that learning occurs within a social environment. Humans develop a relationship between the internal experience of pain, and external events, such that they can recognise and avoid actual or potential harm, and internalise behavioural norms associated with these events. This process begins at birth and continues as individuals mature. Greater voluntary control over expressing distress develops alongside increasingly sophisticated emotion regulation and cognitive development (Noel, Petter, \& Chambers, 2012). Family and community attitudes and typical behaviours are more similar amongst family members than neighbours and amongst communities in one country than communities from another country. Examples of such similarities include pain descriptions, treatment-seeking, illness behaviours and selfregulating responses to painful stimuli (Goubert, Vlaeyen, Crombez, \& Craig, 2011; Hsieh, Tripp, \& Ji, 2011; Ness, 2009; Rollman, 2004; Throop, 2008). Not only do individuals learn about appropriate behaviour with respect to their own pain, they also develop normative responses to pain behaviour in others (Farmer, Roter, \& Higginson, 2006; Gulbrandsen, Madsen, Benth, \& Laerum, 2010). This is a point to which I will return later in this chapter when I discuss health professional attitudes towards the treatment of pain.

A third key concept is the emotional tone of the experience: pain is unpleasant. Pain is an aversive experience associated with negative moods such as sadness, fear and anger, and suffering (Burns, 2006; Hamilton, Karoly, \& Kitzman, 2004; Neugebauer, Galhardo, Maione, \& Mackey, 2009; Wiech \& Tracey, 2009). There are some exceptions such as modern body suspension (Horton, 2012) and body modifying "rites of passage" (Morinis, 1985), but even in these contexts, individuals endure the pain in order to reach some other, more positive goal. Even single-celled organisms without the necessary capacity for 
emotions will attempt to escape and avoid physical harm, but as organisms become more complex, so do the neurobiological structures that underpin responses to threats within the environment (Hadjistavropoulos et al., 2011). Negative emotions activate complex neural networks to prioritise actions needed for survival (Damasio, Everitt, \& Bishop, 1996). In addition, humans have developed self-regulatory skills to intentionally modulate both emotional intensity and the attention-demanding qualities of nociceptive stimuli, but this modulation can only be sustained at the cost of cognitive processing capacity (Baliki, Geha, Apkarian, \& Chialvo, 2008; Ploner et al., 2011; Schrooten et al., 2012; Villemure \& Schweinhardt, 2010). The effect of persistent pain on mood and anxiety is well-known (Asmundson \& Katz, 2009; Huijnen et al., 2010; Nicholas, Coulston, Asghari, \& Malhi, 2009; Tang, Goodchild, Hester, \& Salkovskis, 2010), and including the negative emotional effects of pain in the IASP definition provides a context for clinical efforts to alleviate the distress and suffering of those experiencing pain.

Finally, the definition affirms that pain is inherently a conscious psychological experience rather than simply nociceptive pathway activations. Consciousness is still hotly debated in many disciplines, and commentators often use pain to illustrate "the venerable mind-body problem" (Koch, 2004, p xv). Researchers, clinicians and individuals now consider psychosocial factors are an integral part of the phenomenon, rather than merely a response to a transmitted nociceptive stimulus. This conceptualisation has developed out of differentiating between the experience of having pain, and the neural mechanisms of nociception or information transmitted through the peripheral nerves to the cortex, becoming modulated and in turn generating actions.

Members of the IASP Taxonomy Working Group continually update definitions of pain, although the definition of pain has not appreciably changed since IASP adopted it in 1986. There have been minor amendments to the accompanying note. Some critics argue the definition ignores behaviours associated with pain and confines the focus to learned 
associations between the word and the experience rather than being an innate characteristic of living organisms (Anand, Rovnaghi, Walden, \& Churchill, 1999; Anand \& Craig, 1996; Cronje \& Williamson, 2006; Rollin, 1999; Shapiro, 1999; Wright, 2011). This definition nevertheless dominates the current conceptualisation of pain, and the implications of the definition have led to a body of knowledge that integrates the biological, psychological and social factors associated with the personal yet ubiquitous experience of pain.

\section{Acute and chronic pain}

The most common form of pain is acute pain: this pain lasts for less than three months, reduces in intensity over that time, primarily relates to actual or potential tissue damage, and has an inherent biological function (Loeser \& Melzack, 1999; Melzack \& Wall, 1988). People with acute pain can become distressed or overwhelmed by it, but psychosocial factors are not thought to influence the experience to the same degree as those with chronic or persistent pain (Nicholas, Linton, Watson, \& Main, 2011). Acute pain is generally well-accepted in the community, usually as a symptom of an underlying disease process. People who report acute pain will typically seek treatment, obtain a diagnosis, take analgesia to manage the pain, and temporarily adopt, and be given allowances for, "illness behaviour" during recovery (Main et al., 2008).

Persistent or chronic pain is less clearly defined than acute pain. Although duration (longer than three months), and healing (pain persisting although tissue damage is no longer present) usually guide classification, the Introduction to the Classification of Chronic Pain ( $2^{\text {nd }}$ Edition) points out that neither of these factors represents an absolute point at which a pain moves from acute to chronic (Merskey \& Bogduk, 1994). For an individual to transition from acute to chronic pain involves many considerations. These include: 
- Whether there is any underlying disease process not yet satisfactorily diagnosed and treated (Manchikanti, Singh, Falco, Cash, \& Pampati, 2008). Some clinicians argue that many individuals with chronic pain simply have not had appropriate investigations, and thus have not been given the treatment required to abolish their pain entirely (Jull et al., 2011).

- The "usual" prognosis for this condition (Dasch et al., 2008; Salpakoski et al., 2010).

- Whether there has been any time during this period when pain has reduced or gone altogether (Mullady et al., 2011).

- Whether distinctions should be made between experiencing pain, seeking treatment for pain and experiencing interference in daily activities (Main et al., 2008).

In chronic pain states, the influence of psychosocial factors on disability becomes more prominent over time than the initial biological event, and in view of this shift there are calls for chronic pain to be designated a "disease" rather than a symptom of an underlying problem (Loeser \& Melzack, 1999; Merskey \& Bogduk, 1994; Siddall \& Cousins, 2004). If chronic pain is a disease, it must have a pathology and associated signs and symptoms distinct from acute pain. Siddall and Cousins (2004) outline the cascade of changes from peripheral to central, and affecting numerous body systems and functioning that continue when there is either continuing nociception or arise from disruption of afferent nerves (p. 513) and argue that this justifies the assertion that chronic pain is a distinct disease entity. Alongside the growing discoveries within neurobiology of pain, research is rapidly uncovering the importance of both psychological and social factors. Some of these factors are present prior to pain onset, such as the social milieu and beliefs and attitudes towards pain (McParland, Eccleston, Osborn, \& Hezseltine, 2011; Moldovan, Onac, Vantu, 
Szentagotai, \& Onac, 2009),while others emerge once pain is present to complicate and maintain distress and disability (Blyth, Macfarlane, \& Nicholas, 2007; Molton et al., 2009). Many questions have also been raised about the transition from acute to chronic pain, and I will draw attention to some of them shortly. Within this thesis, I do not discuss tissue pathology or disease processes because my research focuses on individuals who have had any underlying disease process managed effectively but still have pain. The purpose behind considering chronic pain in this way is that the qualities of pain do not differ between acute or chronic pain (i.e. whether the pain is aching, sharp, burning, piercing and so on) (Trudeau et al., 2012), and it is the persistent, and unremitting nature of the pain that participants live with that, is the focus of my investigations, rather than the underlying disease process.

\section{Distinguishing between pain and disability}

Although the experience of pain is an internal subjective phenomenon, the behaviours humans display when they have pain are public affairs. This means they can be interpreted by others, and convey something about the individual's internal experience. Pain behaviours are not always disabling, and disablement does not always involve pain behaviours, although there are links as I will show.

Disability refers to restrictions in being able to perform activities usual for an individual of a similar age while impairment refers to the loss of, or abnormality of psychological, physical, or anatomical structure and function and refers to organs or bodily systems (WHO, 1980). The relationship between impairment, disability and pain is not clear-cut (Martel, Thibault, \& Sullivan, 2010; Severeijns, Vlaeyen, van den Hout, \& Weber, 2001; Waddell, Main, Morris, Paola, \& Gray, 1984). Pain behaviours in acute pain are often useful and adaptive, both signalling to others that the individual hurts, and protecting the individual from exacerbating any underlying pathology (Labus et al., 2003). It is in chronic 
pain states that pain behaviours can become unhelpful, limiting the individual's willingness and ability to engage in normal activities (Martel et al., 2010; Prkachin, Schultz, \& Hughes, 2007; Romano et al., 1988). The difficulty with a division between helpful and unhelpful is that there is no clear transition from one point to another, and this is most evident for the individual experiencing the pain.

Irrespective of the pathology involved, the model of pain followed, or the length of time an individual has been in pain, intra-individual pain behaviours remain consistent although may vary widely between individuals. Individuals develop pain behaviours from reflex responses that social learning and operant conditioning modify, and implicit learning models can shape pain behaviour without the individual being aware of reinforcement contingencies (Holzl, Kleinbohl, \& Huse, 2005).

Of greater importance, in terms of chronic pain and pain behaviour is the finding that selfreported pain intensity and observable pain behaviour correlate more strongly when an individual has acute pain (Labus et al., 2003). This suggests that over time, the relationship between pain intensity and pain behaviour become weaker and some authors argue that catastrophising (thinking the worst), and social reinforcement increasingly influence pain behaviour (Martel et al., 2010).

Historically, interest in behaviourism within psychology led to recognising that pain behaviours could be influenced through reinforcement mechanisms (Fordyce, 1976, 1982b). Experiencing pain and the behaviours associated with having pain were differentiated as a result of this reconceptualisation. Fordyce and colleagues $(1968,1982)$ argued that when pain intensity or quality could not be modified because of limits to medical care, then disability and distress could be influenced by applying behavioural strategies to increase well behaviour through selective reinforcement while removing reinforcement for illness behaviour (Fordyce, 1974; Fordyce, Fowler, \& Delateur, 1968; Fordyce, Shelton, \& Dundore, 1982). 
At that time, chronic pain was considered a physical problem, with psychological aspects of pain mainly thought of as reactions to the physical problem, or, in the absence of any identified physical disorder, psychogenic or functional - in the words of patients: "all in your head". Fordyce developed a framework for understanding how learning processes and social and environmental factors could contribute to suffering and disability (Patterson, 2005). The context for Fordyce's findings was at a North American University Hospital, and referrals to the treatment setting consisted of mainly North American, white, middle-class individuals. Researchers and clinicians considered people with chronic pain to be a relatively homogenous group, with common characteristics of deactivation and depression, and demonstrating a similar range of pain behaviours (Fordyce et al., 1982). Behavioural reinforcement mechanisms explained individual differences in pain behaviours, and at that time, cross-cultural differences were not well-represented in the pain literature.

Subsequent cross-cultural research finds that pain behaviour differs considerably between individuals from different cultural groups. These differences apply not only to groups with various ethnic backgrounds, but also to groups with the same ethnicity but who have formed sub-cultures within a community (Hobara, 2005; Kirmayer, 2008; Rollman, 2004; Wein, 2011). Research studies also show behavioural differences in the same individuals in different contexts, such as at home, in the Emergency Department, or with people from different ethnic backgrounds in the environment (Green, Baker, \& Ndao-Brumblay, 2004; Hsieh et al., 2011). Most of this research has examined differences between groups and cannot fully consider individual differences, and, notably, most research has examined patient behaviour rather than health provider behaviour, with some exceptions (Vlaeyen $\&$ Linton, 2006). If, however, one of the functions of pain behaviour is to communicate distress to others (Hadjistavropoulos, Craig, \& Fuchs-Lacelle, 2004; Hadjistavropoulos et 
al., 2011), the interactions between individuals should also be considered, and in the case of patients, this should examine health professional behaviour.

There is a small body of knowledge about health provider behaviour in comparison with that available about patient behaviour. This may reflect the relatively small amount of time patients spend with health professionals compared with the time spent with a family and community, but it may also reflect a general tendency to focus on the individual experiencing pain rather than considering the individual in the contextual of their life (Montali, Monica, Riva, \& Cipriani, 2011).

To date, studies have found little agreement between health professionals' and patients' assessments of pain intensity and disability (Allegretti et al., 2010; Berry, Wilkie, Thomas, \& Fortner, 2003; Bertakis, Azari, \& Callahan, 2003; Perreault \& Dionne, 2005). The context of the assessment and individual's own experience of pain influence health providers responses. FMRI studies show changes in clinicians neural processing of communicative behaviour as they become more experienced (Akitsuki \& Decety, 2009; Benuzzi, Lui, Duzzi, Nichelli, \& Porro, 2008; Betti et al., 2009; Gauthier, Thibault, \& Sullivan, 2008; Han et al., 2009). There is some suggestion that health professional training results in reducing empathy, rather than enhancing empathy (Murinson, Agarwal, \& Haythornthwaite, 2008). Neurobiological studies of empathy show reduced activity in the somatosensory cortex, insula, anterior cingulate cortex, and periaqueductal gray, areas commonly associated with pain processing when physicians watched other people being pricked by a needle (Riess, 2010). Reiss points out that being able to self-regulate emotions may reduce the risk of personal distress and burnout that could otherwise become negative consequences of constant exposure to others' pain and distress, but at the same time this may also have implications for empathic care (Riess, 2010).

Many articles in the health literature suggest that by providing training in empathy clinicians will provide more compassionate care, achieving better clinical outcomes for 
patients (for example Altschuler (2006); Bensing et al. (2011); Bonvicini et al. (2009); Branch, Davis, and Weng (2012). One commentator disputes this, arguing firstly that the meaning of empathy is unclear and that the "meaning, role and relevance" of empathy in clinical care is assumed rather than having been examined (Smajdor, Stockl, \& Salter, 2011). Empathy and sympathy are distinct from one another: sympathy is concern for another while empathy is appreciating the emotions of another. Smajdor et al. (2011) argue that health practice relies on health practitioners being able to distance themselves from the subjective world of the patient and that this protects both the patient and themselves. Practitioners who empathise in order to satisfy their own needs may be harming the patient (in a voyeuristic way) while excess levels of empathy may lead to over-treating or managing short term distress without necessarily managing the long-term effects of this response.

While practitioners influence treatment response, pain behaviour and treatment-seeking in people with chronic pain, other psychosocial factors also have a profound impact on disability arising from pain. Similar factors appear to affect disability in acute pain (for example, post-surgical pain) as those found to influence disability in chronic pain - such as reporting severe pain, experiencing depression, having psychological vulnerability, chronic stress, delayed return to work (Hinrichs-Rocker et al., 2009), or the tendency to catastrophise and to fear movement (Sullivan et al., 2009).

The only group of individuals with ongoing pain who demonstrate slightly different characteristics are those with cancer pain, perhaps because of the meaning of pain and end of life particularly in palliative care. Even in this group, however, those reporting higher levels of catastrophising, negative mood states, and social isolation present as the most distressed and disabled (Zaza \& Baine, 2002). Very similar variables appear to influence pain and the disability associated with pain, irrespective of its duration or the type of pain. This supports the need to consider not only any tissue pathology or central sensitisation 
involved in transmitting and modulating pain, but also psychosocial variables that strongly influence both the experience and also pain behaviour and disability.

\section{The problem of chronic pain}

I turn now to consider chronic pain in more detail to introduce some of the factors considered crucial for understanding the problems it poses for individuals, communities, healthcare providers and researchers, beginning with a brief summary of the prevalence in New Zealand.

\section{Extent of the problem}

It is not easy to estimate the prevalence of chronic pain in the general New Zealand population, at least in part because definitions of chronic pain vary among studies and because few epidemiological studies are available. A recent study, using data drawn from the 2006/07 New Zealand Health Survey, suggests that between $11-19 \%$ of adults experience persistent pain (Dominick et al., 2011) equating to roughly one person in six over the age of 15 years. This study defined chronic pain as "pain that is present almost every day ... pain that has lasted or is expected to last 6 months or more" (p. 64) and could not verify the diagnoses associated with pain. Researchers did, however, ask participants to identify what they thought had caused their chronic pain, finding that $41.5 \%$ of those with chronic pain believed an injury or accident was associated with their pain; $27.7 \%$ associated it with a health condition; and 11.1\% believed it was age-related (Dominick et al., 2011, p. 68). Two-thirds (67\%) of those reporting chronic pain had lived with the problem for five or more years. Participants in this survey reported poorer mental and physical health than the general population. Over one third of participants indicated they had more than one pain site, and the number of pain sites negatively correlated with their health-related quality of life, as measured by the Short-Form 36, a commonly-used measure of health status (Dominick et al., 2011). 
The findings from this survey vary little from findings in other countries, notwithstanding varying definitions and the methods used to conduct the studies. The authors indicated some prevalence differences across sociocultural groups that merit further study. Asian and Pacific Island ethnic groups reported lower rates of chronic pain compared with European and Maori New Zealanders. The prevalence did not vary significantly by gender and differed from results in many other countries where fewer men report chronic pain than women (Dominick et al., 2011, p. 72).

The impact of chronic pain on human lives and the economy is also difficult to establish. In a 2010 report for Arthritis New Zealand, Access Economics estimated that the combined direct and indirect costs of arthritis alone were $\$ 3.2$ billion or $1.7 \%$ of gross domestic product ("The economic cost of arthritis in New Zealand in 2010," 2010). In view of this work's focus on arthritis rather than chronic pain arising from other causes, this is probably an underestimate of the total cost of chronic pain in New Zealand. Dominick et al. (2011) found negative correlations between socioeconomic status and chronic pain. This has also been established in international studies although causal mechanisms are not well-understood (Brekke, Hjortdahl, \& Kvien, 2002; Chibnall \& Tait, 2009; Eriksen, Ekholm, Sjogren, \& Rasmussen, 2004; Grotle, Brox, Glomsrod, Lonn, \& Vollestad, 2007; Morgan, Conway, \& Currie, 2011; Nguyen, Ugarte, Fuller, Haas, \& Portenoy, 2005).

While the picture of living with chronic pain illustrated as above seems negative and disheartening, this is not the whole picture. In the same New Zealand study, over one third (36\%) of individuals reporting chronic pain did not use any medical treatments for their pain (Dominick et al., 2011). Similarly, other studies have found a high proportion of people with chronic pain manage this without medical or other help. Ohayon and Stingl (2012) surveyed individuals in Germany over the age of 15 years, and from a pool of 3786 eligible participants, 3011 completed a telephone interview (79.5\% response rate). Nearly $28 \%$ of respondents indicated they had pain, with $24.9 \%$ reporting chronic pain (lasting three 
months or more). This study compared those with neuropathic pain features and those with non-neuropathic pain, and found $74.2 \%$ of those with neuropathic pain sought treatment, and of these, $41.6 \%$ received a prescription for medication while $60.5 \%$ of those with non-neuropathic pain sought medical help, and $22.3 \%$ of this group received prescriptions for medication. Thus, between 25.8\% (neuropathic pain) and 39.5\% (nonneuropathic pain) of respondents had not sought treatment for their pain in the previous twelve months.

A Japanese epidemiological study by Nakamura, Nishiwaki, Ushida, and Toyama (2011) obtained responses from 11507 participants over the age of 18 years (60\% response rate). The chronic musculoskeletal pain prevalence was $15.4 \%$, using a definition of pain persisting for at least six months. Of this group, $42 \%$ had sought treatment for their pain, yet only $0.3 \%$ reported that their pain was eliminated through treatment while simple arithmetic suggests around 58\% of respondents did not seek treatment. Data on relationships between pain severity, disability and treatment seeking were not available.

Finally, Karoly and Ruehlman (2006), in a study of 2407 people with chronic pain, identified a resilient group of 320 participants who scored 1 standard deviation or more above the mean on the Severity subscale on Profile of Chronic Pain: Short Form (PCP:S), and less than 1 standard deviation above the mean on both the PCP:S Interference and Emotional Burden subscales. They identified a matched group of non-resilient participants from the same study, and both groups of participants completed the PCP: Extended Assessment. This is a 95 item, 13 scale instrument providing information on pain coping, pain attitudes and beliefs, catastrophising (the tendency toward negative appraisals, rumination and helplessness), social response scales, and additional information on functioning beyond that provided by the PCP:S. Use of treatments (medications, over-thecounter preparations, and other health treatments) were also recorded. The differences between the two groups provide an insight into some of the hypothesised mechanisms 
through which disability and distress may be maintained. The resilient group reported higher levels of belief in their ability to control pain; lower levels of belief in disability, a medical cure and pain-induced fear. They also reported lower levels of catastrophising compared with the non-resilient group. In contrast, the non-resilient group said they received greater amounts of social insensitivity and social impatience as measured by the PCP:EA although there was no difference in the overall levels of social support between either group. In terms of treatment, those identified as non-resilient were more likely to be receiving treatment for pain (78\%) than those identified as resilient (60\%), this pattern continued when prescription pain medications were also analysed, although over-thecounter preparations did not differ between the groups. The authors of this study draw attention to the relatively large group of people $(13 \%)$ in the overall sample who were identified as resilient on the basis of their scores on the PCP:S. They point out that there were significant differences between scores this group obtained on the PCP: EA and scores achieved by the non-resilient group and that these differences applied to the use of treatments by those in the resilient group. They note that differences they might have expected to obtain on the Coping subscale between the two groups were not consistent, suggesting that coping strategies may not be simply dichotomised into "active = good" and "passive $=$ bad".

This brief review of epidemiological studies suggests there are a sizable number of individuals identified in the populations of different countries who, although experiencing chronic pain, do not seek treatment, and many remain relatively non-disabled and undistressed. From Dominick's study (2011) it is evident that a similar proportion of individuals also exists in New Zealand. Distinguishing between having pain and being distressed and disabled by it is crucial to note, and I briefly review historical models of pain that have shaped community attitudes towards pain in the following section. I then discuss selected models used to explain pain, disability and distress. 


\section{Historical models of pain}

There is no distinction between pain of the body and emotional pain or pain of the soul in early historical accounts of pain; for example records from ancient Egypt show that demons were believed to cause pain (Karenberg \& Leitz, 2001). Later in Egyptian history, incomplete digestion of food was supposed to generate "pain-matter" that went through the body and caused painful ailments (Karenberg \& Leitz, 2001). Even later, in the Fifth Dynasty (2450 BC), Egyptians believed that body mixtures such as fluids and gases needed to be "in the correct state of continuity". III health was thus due to "loss of continuity" and treatment aimed to rid the body of substances interfering with continuity through blood-letting, administering enemas, lancing pus, or coughing sputum. Prayers or spells were also administered (Ansary, Steigerwald, \& Esser, 2003). A range of analgesics were used including opium, Salix alba (willow bark), and by mixing calcium carbonate with acetic acid which, in the presence of heat, generates carbon dioxide and provided cooling (Ansary et al., 2003).

Aristotle (348 - 322 BC) believed the heart to be the seat of feelings, and classified pain as an emotion or "passion of the soul", in contrast to Galen (130 - 201 AD) who studied in Alexandria, who believed the brain was the "organ of feeling" and classified pain as a sensation. Galen was the first to classify pain in terms of its sensory qualities, such as stabbing, shooting, and burning (Ansary et al., 2003).

According to Ansary et al. (2003), the Islamic period in Egypt linked the ancient Egyptian and Greek concepts of pain and pain management with the modern renaissance period. Galen drew upon ancient Egyptian treatments such as applying heat, using enemas, as well as analgesic agents while Ibn-Sina (the chief physician of the Islamic world) used drugs to induce sleep and reduce sensation (e.g. opium as analgesic, mandrake and hyoscine as hypnotics), as well as cautery (heating an old nail, covering the wound with a 
castor oil leaf and preferably creating pus, which could be expressed, a reference to earlier beliefs about pain as a break in the continuity of the body) (Ansary et al., 2003).

In many so-called "primitive" cultures, pains that were not directly associated with visible injury, were believed to involve magic fluids, evil spirits, or pain demons intruding into the body, and people used charms or spells to ward off the pain demons, resorting to "medicine men" or healers to exorcise the demons if the pain persisted (Bonica \& Loeser, 2001). Pain was the result of spiritual inattention, deliberate commission of sin, or evildoers attacking the individual on both a physical and spiritual plane. There was no particular division between spiritual, emotional or physical pain, and treatment involved appeasing the offended deity, prayers for mercy, applying herbs, and inflicting wounds to release fluids or vapours, or to balance energies (Bonica \& Loeser, 2001). People also used exercise, heat, cold, massage, diet and in China, acupuncture and moxibustion (burning mugwort (moxa) close to, or on the skin at acupoints).

It was not until the Middle Ages in Europe that a shift emerged towards viewing the brain as the centre of sensation. When Descartes (1596 - 1650) argued for a division between body and soul the move separating physical pain from spiritual or emotional pain was complete, at least in Western civilisations (Bonica \& Loeser, 2001). Although some view this as a retrograde step because of the subsequent problems created by this stance (that is, difficulty accepting pain without clear evidence of physical damage), it is worth recognising that that by separating soul as the domain of the Church and body as the domain of Science, Descartes discovered a great deal about human anatomy.

During the nineteenth and twentieth century, scientific experimentation supported physiological models of pain. Progress with physiological research led to researching sensation and pain, with Müller's 1840 thesis proposing that the brain played a primary role in receiving information via sensory nerves; Müller also identified the five senses although he believed that sensations were transmitted, without modification, from the 
body to the part of the brain responsible for sensation (Bonica \& Loeser, 2001, p. 7). Three schools of thought emerged during the mid- to late-1800's regarding the nature of pain: specificity theory in which specialised nerve fibres were thought to transmit pain, independent of other senses; summation theory in which any stimulus could produce pain given sufficient intensity; and finally, the "traditional" view held since Aristotle, that pain was an affective, or emotional quality. Philosophers and psychologists primarily held this latter belief, believing that pain contributed to motivation (Bonica \& Loeser, 2001, p. 8). These competing views were finally, in 1895, brought together by Strong, the president of the American Psychological Association at the time. He proposed that pain consisted of two parts: the physical sensation and the psychic reaction to this sensation (p. 8).

Researchers maintained this view until 1940's, thinking that pain could be separated into the perception of pain, and the reaction to pain. The model proposed that perception of pain was a neurophysiologic process involving relatively simple and primitive neural mechanisms while the reaction to pain was a complex physiopsychological process involving cognitive, learned experience, culture and other psychological factors to produce a variable response pain threshold (Bonica \& Loeser, 2001). While psychosocial factors were incorporated into the model, neurophysiological processes involved in transmitting "pain signals", or nociception were believed to be the primary mechanisms. This model held sway until the mid-1960s when Melzack and Wall published their seminal work, Gate Control Theory of pain. This theory held that neural impulses were transmitted to the dorsal horn of the spinal cord, were then modulated via descending inhibitory fibres to "gate" the amount of information forwarded to the brain (Melzack \& Wall, 1967).

There have been significant revisions to the model since then, but the key innovations of this theory instigated reviews of the importance of psychological and social factors. Rather than processing information after the fact, the brain was seen as actively involved in selecting and modifying neural input from the periphery, and as Melzack states 
Pain processes do not begin with the stimulation of receptors. Rather, injury or disease produces neural signals that enter an active nervous system that (in the adult organism) is the substrate of past experience, culture, and a host of other environmental and personal factors. These brain processes actively participate in the selection, abstraction, and synthesis of information from the total sensory input. Pain is not simply the end product of a linear sensory transmission system; it is a dynamic process that involves continuous interactions among complex ascending and descending systems (Melzack \& Katz, 2013, Abstract).

Research into psychosocial aspects of pain has flourished since the 1965 Gate Control Theory, beginning with the emergence of behavioural learning theories during the mid1970s. Behavioural theories differentiated pain as an experience from behaviours associated with the experience (Fordyce, 1976, 1981). This model of pain introduced pain behaviours as a key target for therapy in those individuals with chronic pain. It was through this model that greater understanding grew with respect to social modelling, familial patterns in pain behaviour, and the influence of social factors such as compensation payments for those people disabled by their pain. Researchers have identified that respondent and operant conditioning influence the frequency and type of pain behaviours that individuals demonstrate when they experience pain. From birth, as individuals observe others and their behaviours are reinforced, pain behaviours gradually conform to fit within sociocultural norms (Hadjistavropoulos et al., 2004).

Cognitive models appeared with respect to pain and disability as interest in behaviourism was gradually supplanted by interest in cognitive mechanisms in both normal behaviour and psychopathology. Attention, or the "mechanism by which sensory events are selected and enter awareness" (Legrain et al., 2009), involves both top down and bottom up processing. Legrain posits that top-down processing involves modulating the sensitivity of stimulus-specific neural responses according to consciously chosen goals; this process 
prioritises information needed for current actions. Bottom-up processing involves unintentional capture of attention by events themselves, depending on their salience (novelty or contrast with other contextual events). The two processes are not independent, with top-down processes influenced by attentional capture from bottom-up processing, resulting in reduced task performance; while bottom-up processing can be influenced by overall attentional load (the amount of information processed at that time) and attentional set (the prioritised features that are similar to stimuli relevant to the task or goal) (Legrain et al., 2009). These discoveries help explain the varying responses individuals can have to injury and pain, depending on context. For example, during a rugby match actions required to score a goal are prioritised over attending to knocks, bruises and even broken bones until after the game.

Similarly, increased sensitivity towards epigastric pain can be found in individuals who have recently had a myocardial infarct and continue to have angina. Individuals would ordinarily be likely to think that epigastric pain is trivial and nonintrusive while after a myocardial infarct, any pain or discomfort in this area dominates attention (Eifert et al., 2000; Zvolensky, Feldner, Eifert, Vujanovic, \& Solomon, 2008). Consequently, it is not surprising that previous experiences of pain influence neurobiological processing when an individual is anticipating pain, influencing his/her emotional state, sympathetic arousal and even activating the endogenous opioid system (Tracey \& Mantyh, 2007). Quartana, Campbell and Edwards (2009) are among some of the authors who suggest that genetic and epigenetic vulnerability may underpin the tendency to catastrophise, thought to be one of the most influential psychological factors associated with recovering from pain (Quartana, Campbell, \& Edwards, 2009). Catastrophising involves negative emotional/cognitive processes including rumination and pessimism, perceptions of helplessness, and magnification of pain-related symptoms (Quartana et al., 2009). Studies have shown that individuals with high levels of catastrophising show enhanced activity in 
regions of the brain associated with emotional and motivational processing, and reduced activation of areas related to pain inhibition (Edwards et al., 2009). Other researchers, such as Sullivan (2008), have shown that catastrophising may be a form of social communication. Individuals who catastrophise also demonstrate higher levels of pain behaviour and this may be associated with efforts to obtain interpersonal support for their pain or stress (Hadjistavropoulos et al., 2011; Lackner \& Gurtman, 2004; Sullivan, 2008).

\section{Models of pain}

Thus far, I have discussed definitions of pain, reviewed the extent of the problem of chronic pain, and looked at a number of models of pain, including briefly discussing the ways individuals communicate their experience of pain. At this point, I will introduce two models of pain that attempt to explain differences in the degree of disability between two individuals with the same level of tissue damage. I have selected these models because they are seminal works that integrate psychosocial factors with biological factors into a coherent explanation for disability associated with pain.

\section{Loeser's "Onion ring" model}

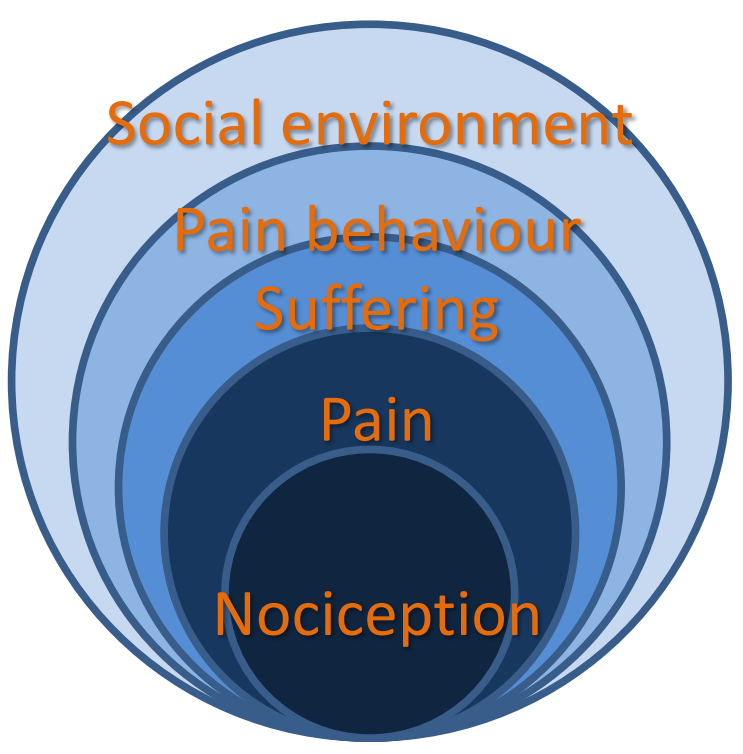

Figure 2. Multi-faceted model of components of pain, Loeser (1982). 
The first is often called the "onion ring" model, developed by Loeser (1982). The model consists of concentric rings, with nociception in the centre. Surrounding nociception is the experience of pain - the personal, subjective aspect of "what it feels like" as discussed at the beginning of this chapter. Surrounding this is a circle representing suffering, and surrounding this is the final circle representing pain behaviour. Some authors have included a further circle indicating the context or the social environment.

Loeser (1982) contended that each ring within the model influences the other rings and that all of the rings exert an influence on the level of disability and distress of an individual. The model integrates biological aspects within the nociception ring, and Melzack's neuromatrix (Melzack, Coderre, Katz, \& Vaccarino, 2001) and other cortical and subcortical biological systems can be incorporated into this ring, although these were not known at the time Loeser (1982) developed this model. Incorporating these latter concepts would allow the model to extend to conditions such as fibromyalgia, post-stroke pain and migraine, which have varying elements of nociception involved.

The second ring, pain experience, cannot be shared directly with another. The degree of biological disturbance strongly influences the experience, along with the third ring which indicates suffering, or the unpleasant emotional response generated in the higher nervous centres of the brain (Moskovitz, 2011). This ring can also be interpreted as the emotional result of judgements or appraisals individuals make about the meaning of a particular pain at a given time. Cognitive therapeutic approaches target appraisals an individual makes with respect to their experience (Day et al., 2012); thus this ring is one of the targets for psychological treatment. Therapists using Acceptance and Commitment Therapy (ACT) try to influence these two rings differently (Hayes \& Duckworth, 2006). ACT focuses on nonjudgemental awareness of the experience represented by the second ring rather than directly modifying appraisals, and helps individuals to recognise that their thoughts do not necessarily reflect reality (Hayes, Luoma, Bond, Masuda, \& Lillis, 2006). Within the context 
of Loeser's (1982) model, in ACT cognitive defusion strategies help to reduce the global and inflexible cluster of emotions and thoughts associated with the experience, reducing the tendency to remain focused on the emotional impact of pain represented by the third ring.

Loeser's model identifies pain behaviour as the only aspect of pain that is available to others (Loeser, 1982). This representation shows pain behaviours resulting from all three previous factors, and by adding the fourth and final ring denoting social environment, show they are also subject to the behavioural mechanisms identified by Fordyce (1985). Fordyce (1985) argued that behaviour provides the only way for another individual to recognise another's "otherwise unknowable experience" (Fordyce, Roberts, \& Sternbach, 1985, p. 114) and that behaviour can be modified. He pointed out that contingencies within the environment influence the persistence of certain behaviours while reducing the frequency of other behaviours; this is operant conditioning. He also discussed the relevance of respondent, or Pavlovian conditioning, although much of his writing focused on operant conditioning, and particularly on social contingencies such as responses from partners and family, treatment providers and people within the workplace (Fordyce, 1974, 1976, 1982b, 1988; Fordyce et al., 1968). Fordyce was clear that alleviating pain was not the goal of a behavioural approach, suggesting that the method be considered a treatment for "excess disability". He believed that the major problem for people with chronic pain was that they were "more functionally disabled than is necessary" (Fordyce et al., 1985, p. 121).

Loeser's model does not incorporate individual values and goals, thought to provide direction and motivation for voluntary behaviour (Austin \& Vancouver, 1996; Freitas \& Higgins, 2002; Freitas, Liberman, \& Higgins, 2002; Grahn, Ekdahl, \& Borgquist, 2000; Higgins, 1997; Paez-Blarrina et al., 2008), and hence this is a weakness of his model. Recent models of pain address this fault by incorporating individual's values and goals to explain motivational aspects of pain. The biopsychosocial model of pain communication 
by Hadjistavropoulos et al. (2011), which I will briefly discuss, is an example of one such model.

\section{Biopsychosocial model of pain communication}

This model is a complex model that incorporates neurobiological, psychological and social influences on pain communication. The authors believe that by understanding these processes in greater detail, the sociobehavioural factors influencing both the person with pain, and individuals involved in caring for, or interacting with that person will be more appreciated (Hadjistavropoulos et al., 2011, p. 2). They argue that pain communication evolved within human society along with language and nonverbal signals but that clinical training programmes pay relatively little attention to the relevance of pain communication, leading to clinicians misinterpreting nonverbal pain behaviour (especially in those with dementia). They hope the model will help clinicians and researchers investigate how and why people show they have pain, and how observers respond to different ways of showing pain. The model derives from a general communications framework, in which communication is categorised into three types: communication as action (or expression), communication as interaction, and communication as transaction. Each of these categories of communication may be used when an individual experiences pain in the presence of another, enacting different effects depending on the intention of the person and the observer, clarity of message encoding, transmission and decoding. Understanding pain communication is critical because pain is a subjective experience that must be represented symbolically in order for others to comprehend it. In the opinions of these authors, communication is a social behaviour. It incorporates both intentional and unintentional actions within a social context of "hidden and explicit rules, assumptions, and a broad[er] social context of expectations and constraints" (Hadjistavropoulos et al., 2011, p. 4). Processes underpinning (a) the internal experience of pain consisting of cognitive, affective, behavioural and motivational components, influenced by contextual 
factors such as culture, family behavioural norms, beliefs about the meaning of pain; (b) encoding the experience within expressive behaviour, bearing in mind that this behaviour may be communication as action (that is, a direct message from the individual about the internal experience) but it may also be communication as transaction (that is, an act designed to influence the observer, interpreted in line with his or her own normative understanding of what those actions represent); and finally, (c) decoding pain expression by one or more observer, also influenced by contextual, cultural, situational and social factors such as age, gender, social status, and even physical attractiveness. These authors point out that both parties within a communication dyad bring their own intentions to the situation and because there is room for inaccuracies in encoding and decoding actions and no way to ascertain whether an individual intentionally encodes or decodes in a particular way (that is, that the veracity of the message encoding or decoding cannot be determined), the purpose for communicating pain must be considered.

Further to this model, earlier discussions by Van Damme et al. (2008); and Van Damme, Legrain, Vogt, and Crombez (2010) suggest that motivation to achieve personal, or life goals must be incorporated when thinking about the ways people cope with and attend to pain. In other words, the context in which individuals experience pain must be considered, as do the context and function of pain behaviours. They point out that while there is value in attempting to control or solve the problem of pain, notably in the acute stage (Keefe, Rumble, Scipio, Giordano, \& Perri, 2004), there is also evidence that this approach can have negative consequences in the event that pain cannot be controlled (Eccleston $\&$ Crombez, 2007). For example, resting is a helpful approach when in acute pain to solve the problem of exacerbating pain intensity, but this strategy can lead to disability, loss of function and withdrawing from activities that bring reward and social support when used in the context of chronic pain (Westman, Boersma, Leppert, \& Linton, 2011).

\section{Pain-related fear and avoidance model}


The third model I will discuss in detail is the fear avoidance model first introduced by Lethem, Slade, Troup, and Bentley (1983), and earlier suggested by Philips and Hunter (1981), but more commonly associated with the work of Johan Vlaeyen and colleagues at Maastricht University (Vlaeyen \& Linton, 2000, 2012; Vlaeyen et al., 1999; Vlaeyen, KoleSnijders, Boeren, \& van Eek, 1995; Vlaeyen, Kole-Snijders, Rotteveel, Ruesink, \& Heuts, 1995).

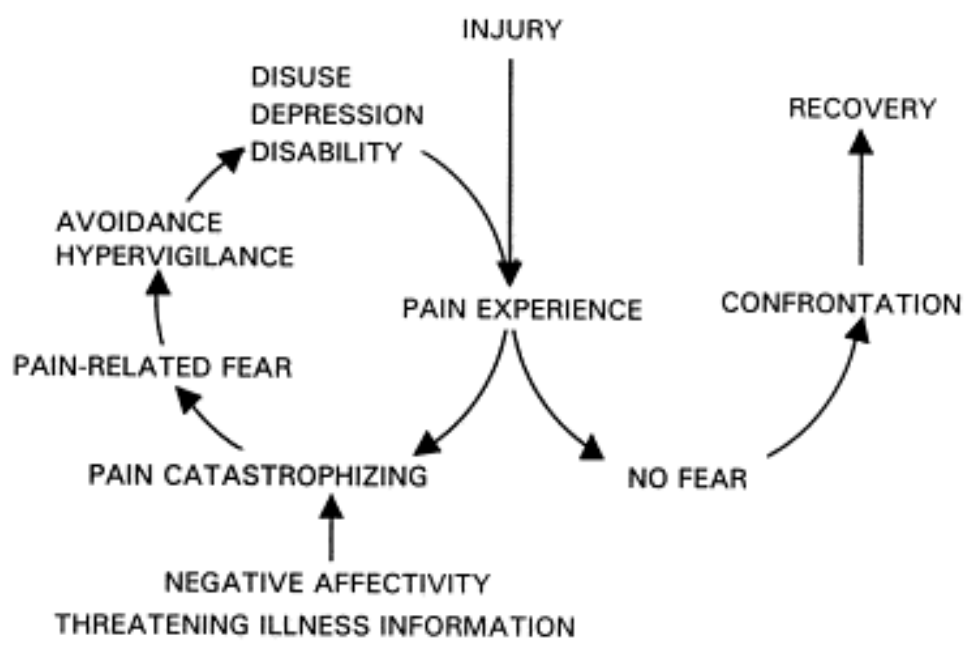

Figure 3. The "fear"-avoidance model developed by Vlaeyen and Linton (2000).

This model is one of the most influential clinical models in use today. It is particularly relevant for explaining the mechanisms by which some individuals develop disability, while others do not. Based on a similar conceptualisation to simple phobias, the model shows that as individuals interpret their experience of pain, some, perhaps those with a greater tendency for negative affectivity, may catastrophise, or "think the worst" about their experience. Individuals, having interpreted their pain catastrophically, become fearful and hypervigilant about their experience of pain. They then avoid situations and movements involving the risk of experiencing pain, subsequently becoming increasingly likely to develop disuse, depression and disability. In turn, this feeds back into the 
experience of pain, only to be once again interpreted as threatening, perpetuating the negative cycle.

The alternative trajectory involves an individual experiencing pain, but not developing fear, thus being able to confront movements and situations, ultimately allowing full recovery.

In a recent review of this model, some twelve years after the last review by the same authors, Vlaeyen and Linton (2012) point out that although there are many empirical studies supporting this model, there has not, to date, been a satisfactory explanation as to how pain-related fear occurs in the first place (p. 1144). Additionally, Vlaeyen and Linton (2012) show that certain features of chronic pain and pain-related fear differentially influence avoidance. For example, there are multiple pathways through which individuals develop fear: (1) direct experience, where pain is considered to be an unconditioned stimulus that activates a defensive response, causing the person to associate neutral cues (or the conditioned stimulus) with the unconditioned stimulus, or pain. A conditioned response (avoidance, as well as increased physiological arousal and negative emotions) is then elicited in the presence of the conditioned stimulus, without the need for pain to be experienced. An example of direct experience occurs after accidentally touching an electric fence used to contain cattle, when subsequent encounters with similar fencing will often produce fear and reluctance to go near the fence.

(2) vicarious learning, or learning from the responses of other individuals, can also provoke fear of a stimulus thought to elicit pain, such as when seeing another person receive a shock from an electric fence, leading to similar reluctance to approach the fence.

(3) verbal information, such as being told that the wire fence will give an electric shock, with similar consequences to the two previous conditions. Vlaeyen and Linton (2012) suggest there may be interactions between the different learning pathways, such that previous verbal warnings about the electric fence, followed by receiving a shock, may enhance the learned response. They also discuss the role of unpredictability in the 
generation of pain-related fear and avoidance, pointing to the distress in individuals with chronic widespread pain rather than these individuals specifically avoiding certain activities or movements. Vlaeyen and Linton (2012) suggest that this could occur because it is more difficult for individuals with unpredictable pain to develop safety behaviours that predictably reduce or avoid eliciting pain; in some individuals this can lead to learned helplessness (Samwel, Kraaimaat, Evers, \& Crul, 2007).

One of the underlying factors for pain-related fear is catastrophising, or the tendency to be negatively oriented towards actual or potential pain experiences (Turk \& Wilson, 2010). Post-operative pain intensity and length of hospital stay have been correlated with presurgical measures of catastrophising (Witvrouw et al., 2009), and higher scores on a measure of catastrophising when combined with a depression score predicted neckrelated disability following acute whiplash-associated disorders (Schmitt et al., 2009). It has also been associated with poorer adjustment to chronic pain in those people with spinal cord injury (Molton et al., 2009; Nicholson Perry, Nicholas, Middleton, \& Siddall, 2009). Catastrophising may be a cognitive appraisal bias, although studies have shown that it may influence an early phase of central sensitization or pain windup (Borkum, 2010; Latremoliere \& Woolf, 2009).

Borkum (2010) suggests that catastrophising may decrease the activity of descending pain-reduction pathways (such as endorphin release), or, by increasing descending pain mechanisms. He argues that self-efficacy for pain coping increases endorphin production while catastrophising increases the sense of helplessness thought to be the inverse of self-efficacy. The exact mechanisms through which catastrophising influences the experience of pain is unknown, although Borkum considers it is likely that catastrophising influences attention to pain, decreasing activity associated with controlling or downregulating pain intensity while it also activates motor control „, enhancing escape preparation (Borkum, 2010). Catastrophising may, therefore, provide adaptive benefits in 
acute pain by enhancing attention and escape, thus providing one pathway for learning to avoid danger, but at the same time can be unhelpful when exaggerating the threat value of the stimulus. Some commentators also conclude that these psychological processes increase individuals' attention to ambiguous sensations that they subsequently misinterpret, gradually broadening the range of feared sensations (Turk \& Wilson, 2010). Escaping or avoiding activities then prevent individuals from recognising that these activities may not increase pain, reducing opportunities for accurate feedback about the threat value of the activity. Fear and avoidance thus are maintained and ultimately extend beyond any original, adaptive function.

Turk and Wilson (2010) criticise the fear avoidance model because fear, an emotion, and the behaviour, avoidance are conflated (Turk \& Wilson, 2010). This is especially notable because studies show that graded exposure aimed at reducing avoidance does not eliminate fear, and instead promotes new behaviour: to approach the feared stimulus (Day et al., 2012; Vlaeyen \& Linton, 2012). Other concerns with the model lie with reliance on self-report measures of pain-related fear on the basis that these assume individuals are fully aware of the reasons for being fearful of, and catastrophising about, pain, although this is not always the case (Vlaeyen, Morley, Linton, Boersma, \& de Jong, 2012a). Turk and Wilson (2010) also suggest that the model fails to explain pain with insidious onset and that some individuals do not avoid activities because they fear further damage, but rather, they fear the pain itself. Turk recommends further research into these aspects of avoidance because they may need different approaches during treatment (Turk \& Wilson, 2010).

Finally, Vlaeyen and Linton (2012) join with researchers such as Asmundson (2012); Crombez, Eccleston, Van Damme, Vlaeyen, and Karoly (2012); Lauwerier et al. (2012); PaezBlarrina et al. (2008); Smith, Tooley, et al. (2009); Van Damme et al. (2008); Van Damme et al. (2010); Van Damme, Van Ryckeghem, Wyffels, Van Hulle, and Crombez (2012) in 
considering the contribution of goals and contextual issues on the way pain-related fear develops. Avoiding pain is only one goal amongst the many goals individuals may pursue, and there are numerous examples where people willingly undergo painful procedures for health, beauty or social acceptance (e.g. hip arthroplasty, cosmetic surgery and tattoos, to list just a few). There are also many individuals who prefer to avoid treatments such as hip arthroplasty despite ongoing pain because they anticipate increased pain during the immediate post-operative period (Dolin et al., 2003; Zikmund-Fisher et al., 2010). Despite recognising that individuals may have a range of goals, only one of which is reducing pain, health professionals and particularly medical practitioners seem obligated to offer pain reduction or abolition as the priority for individuals seeking help with their pain.

Discussions about the goals individuals actually have, in comparison with those health professionals assume they have, happen less often than discussions about how pain should be reduced or abolished; while the media seldom questions community attitudes towards the need to get rid of pain, at least in affluent Western society. I will discuss this in a later section, but before I do, I will discuss pain management for those who have chronic pain.

\section{Introducing social context}

While pain is unpleasant, and searching for relief a reasonable and even necessary step for individuals to take, there are some contexts in which pain is not only tolerated, but invited. Endurance athletes (Kress \& Statler, 2007), Army recruits (Harper, 2006), and boxers (Downey, 2007) are amongst those encouraged to cope with pain. "Pain is weakness leaving the body" is an expression thought to have originated in the Marine Corps during the late 1960's, but still found in the New Zealand Army today (K. Lalahi, personal communication, 20 May, 2012).

Body suspension, body piercing, and tattoo are all painful procedures that have origins in ancient cultures, and may represent an ordeal through which adolescents declare their 
willingness to assume adult responsibilities (Cote, 1997). The re-emergence of Maori and Pacifika tattooing in recent years is a statement about the relevance of cultural values and practices of these people in New Zealand society (Museum of New Zealand Te Papa Tongarewa, 2011). A group formed in Christchurch describes body suspension this way: The act of suspension is hanging the human body from (or partially from) hooks pierced through the flesh in various places around the body.

There are many different reasons to suspend, from pure adrenaline or endorphin rush, to conquering ones [sic] fears, to trying to reach a new level of spiritual consciousness and everything in between. In general, people suspend to attain some sort of "experience".

Some people are seeking the opportunity to discover a deeper sense of themselves and to challenge the pre-determined belief systems which may not be true. Some are seeking a right [sic] of passage or a spiritual encounter to let go of the fear of not being whole or complete inside their body.

Others are looking for control over their body, or seek to prove to themselves that they are more than their bodies, or are not their bodies at all. Others simply seek to explore the unknown. Retrieved from http://www.suspension.org/hooklife/about/ Monday, 17 September 2012.

The social context informs individuals' attitudes towards the pain experience and also contributes to both the expression of pain behaviours, and the use of coping strategies.

\section{Attitudes towards pain}

From the pain of body suspension to the pain of childbirth, there are examples of intense pain that can be managed, even embraced. There are many reasons given for how and why people can tolerate pain in these situations. Role socialization, for example, when dealing with a bruising encounter during a wrestling match (Curry \& Strauss, 1994); or 
wanting to fit in, such as when participating in physical training in the Army (Harper, 2006), with the ultimate goal being more valuable than the current pain. Pain is not a straightforward stimulus-response experience. Not only do individuals alter their own response to nociception; society and community beliefs about the experience alter over time, as attitudes towards pain in childbirth illustrate.

\section{Attitudes toward pain in childbirth}

The history of pain relief in childbirth reveals the changing attitudes of people from within the community, healthcare, and religion. Even before Hippocrates advised women to chew willow bark to alleviate the pain of childbirth, women used breathing, massage, herbs, physical positioning and having social supports to help them cope with labour pain (Leap \& Anderson, 2004). These supportive attitudes changed under the influence of both religious teachings and increasing medical professional dominance. Woolcock, Thearle, and Saunders (1997) provide an example in the context of colonial Queensland society, when they recount the common attitude that the pain of childbirth represented the curse of Eve, and thus it was a woman's place to suffer. People used the Biblical passage in Genesis 3:16 to justify this attitude: "I will make your pains in childbearing very severe; with painful labour you will give birth to children" (New International Version) (Woolcock et al., 1997).

Expectations towards obstetric pain changed after Sir James Young Simpson $(b .1881$, d.1870) introduced chloroform in 1847. He is quoted as saying "All pain is per se and especially in excess, destructive and ultimately fatal in its nature and effects", and he apparently favoured general anaesthesia in midwifery for every delivery (Pearce, 2008. "The case for pain", para. 7). In the 1920's medical practitioners administered a mix of Nembutal and Scopolamine (Hyoscine) to the labouring woman, giving her a twilight sleep, in which she was semi-conscious and unable to remember the pain once she had given birth (Stojanovic, 2008). The tide turned away from twilight sleep in the middle of the $20^{\text {th }}$ 
century when Grantly Dick-Read popularised natural childbirth. He defined labour pain as "a psychic stimulus, reproduced from misconceptions based upon culture". He supported this notion by his reading of the anthropological literature from the late $19^{\text {th }}$ century, in which he claimed "primitives experienced easy, painless labours" (Moscucci, 2003, p.171). He went on to say "...labour was regarded as nothing more than "hard work" in the struggle for existence." He believed "a number of cultural factors conspired to distort a woman's natural capacity for painless birth, producing in women a fear of childbirth that hindered normal parturition." From this, he generated his educational approach for birthing women so they could be "tactfully, gradually and carefully initiated into the job they were about to perform" (Dick-Read as cited in Moscucci, 2003, p. 172). This is an altogether different perspective from that promulgated in the Book of Genesis.

Callister and colleagues (2003) described the perceptions of pain in childbirth in a group of culturally diverse women, finding that women "made meaning of their pain experience" (Callister, Khalaf, Semenic, Kartchner, \& Vehvilainen-Julkunen, 2003, p.149). They suggest "searching for meaning may become a powerful coping mechanism for the woman giving birth" and go on to quote Grainger and McCool (1998, p.256) saying "Birth is an important emotional and spiritual growth process, and enduring labour pain may be an integral part of that growth" (Callister et al., 2003, p.151).

In New Zealand, many women attend ante-natal classes to prepare for birth and discuss labour. Women develop a birthing plan documenting the woman's preferences for pain management in collaboration with their midwife. Midwifery as a profession considers birthing is a natural process and supports a woman's choice to give birth without pain relief (Lee Davis \& Walker, 2011). Even low risk women with midwife support, however, have an increased risk of receiving pharmacological pain management when giving birth in a secondary hospital setting, and this risk increases if giving birth in a tertiary hospital (Davis et al., 2011). The practice of providing pain relief for labour pain clearly varies 
depending on healthcare setting and the professionals involved, suggesting that there are distinct differences between different professionals, and indeed amongst women, in relation to the perceived need for pain relief and a woman's ability to cope with this experience. Attitudes towards a woman's ability to cope with intense pain have shifted over time, place and cultural, and it is noteworthy that Callister and colleagues point out "a painful birth is just as likely to have a positive evaluation as one without pain, depending on the woman's feelings of fulfillment" (Callister et al., 2003, p.151).

The recent history of obstetric analgesia illustrates the enormous influence of sociocultural factors on the way humans experience pain, and the responses to this experience. There is no fixed one-to-one relationship between input and experience; the experience of pain and subsequent behaviour associated with it are highly dependent upon both intrapersonal and contextual factors. Modern chronic pain management programmes exploit these aspects of pain when encouraging cognitive and behavioural strategies to enhance coping.

\section{Ethics and managing pain}

The four principles of medical ethics obligate medical practitioners to respect (1) autonomy, the right of patients to control what happens to their bodies; (2) beneficence, the responsibility to do the most good for the patient in every situation; (3) nonmaleficence, to avoid causing harm, including where a treatment intended for good, unintentionally causes harm; and (4) justice, to be as fair as possible when offering treatments to patients, or allocating finite health resources (Gillon, 1994). The principles are intended to guide clinicians when determining appropriate healthcare choices, yet while there is little argument about the value of these principles, they do not provide a methodology for resolving situations where there are conflicts between the principles. In chronic pain management, as I will demonstrate, there are a great many ethical dilemmas. 
Discussions about medical ethics often incorporate concepts such as dignity, suffering, pain, anguish and fear (Pellegrino, 2011; Pullman, 2002). Ethical care aims to preserve an individual's dignity and alleviate suffering by delivering healthcare, although painful procedures may be required to do so while results may not always result in improved quality of life (Bourne, Chesworth, Davis, Mahomed, \& Charron, 2010; Bozic et al., 2010). As Pullman states:

Unmitigated pain and suffering are often thought to rob human beings of their dignity.... on the other hand, how one responds in the face of pain and suffering is often thought to contribute to one's sense of dignity...it could be argued that the desire to live in spite of pain and suffering expresses and enhances dignity (Pullman, 2002, p. 75)

\section{Suffering}

The concepts of pain and suffering are often considered synonymous. I have already discussed a definition of pain, and briefly touched upon suffering when considering the Loeser model of components of pain (Loeser, 1982). Pain does not always involve suffering: consider the professional boxer, athlete in training, or individuals undergoing body suspension. Cassell (2011) points out that pain intensity alone is only one factor in the distress a person feels saying "people will tolerate even very severe pain if they know what it is (its significance), and if they know that it will end" (p. 10).

One definition of suffering refers to "a perceived loss or threat of loss of the integrity of personhood" (Chapman \& Gavrin, 1999; Moskovitz, 2011, p. 35). Personhood, according to Moskovitz, consists of a composite of memories and preferences, or the autobiographical self. It is the threat to the self, or the person I am (Morley, Davies, \& Barton, 2005; Sutherland \& Morley, 2008) that is fundamental to the concept of suffering (Cassell, 2011; Chapman \& Gavrin, 1999). When an individual begins to recognise that the favoured activities, beliefs, and pleasures of the past, or the future pathway, as it was envisaged, is 
disrupted, he or she can become distressed, until, as Cassell points out, "the threat is gone or intactness or integrity is restored" (Cassell, 2011, p. 10). Cassell distinguishes between suffering and pain: suffering occurs "to the whole person", but the particular aspects of one individual's situation that provoke suffering for an individual will be different from another's (Cassell, 2011). What this means is that while two people may have the same pain problem (diagnosis) with the same pain intensity, the same pain quality, and they may even suffer to the same extent as one another (if this could be measured), the threat to the self that provokes suffering could be quite different. To one, it may be treatment appointments that disrupt daily life causing most suffering while to another it may be the loss of financial security and plans for the future that has the greatest impact on selfidentity.

Chapman and Gavrin (1999) describe a model of the relationship between pain and suffering where pain contributes to suffering through its effect on function. An individual's sense of self develops through past achievements and future goals. The onset of chronic pain and consequently reduced ability to do what is important in the present and potentially for the future challenge an individual's self-concept. The person can feel that he or she is less capable than they were in the past and therefore less than they should be. The "disparity between what the person believes himself or herself to be and what the person is with chronic pain ... represent[s] a threat to the integrity of the self, not only in the present but also in the future ... This damage ... is the essence of suffering" (Chapman $\&$ Gavrin, 1999, p. 2236).

Chapman and Gavrin believe that medical practitioners use the term suffering in a similar way to that of the layperson, that is, that suffering is equal to the "perception of damage to the self and grief over a loss in self-esteem" (p. 2236). Suffering may be inferred (incorrectly perhaps) by a clinician observing a patient's distress as it is expressed in nonverbal behaviour as I will discuss shortly, but Cassell considers that appropriate 
assessment of suffering must include questions such as "Are you suffering?", "I know you have pain, but are there things that are even worse than just the pain?", "Are you frightened by all this?" "What is the worst thing about all this?" (Cassell, 1999, p. 532). In asking these questions, the clinician can become aware of the meaning of symptoms (including pain) in relation to self-identity, enabling more adequate attention to these aspects of illness than indicated by only addressing symptom management.

While clinicians may confuse suffering with grief, loss of self-esteem, or even distress associated with pain, Cassells argues that suffering always involves self-conflict between what an individual wants and what is present; that it always involves a loss or change in life purpose, from being oneself to removing the source of suffering; and finally, that suffering is lonely because (1) of the individual nature of the elements within the situation that causes suffering; (2) that only the individual experiences it and (3) because the person who suffers withdraws from their previous life purpose and connections (Cassell, 2011, p. 11). At least part of the loneliness of suffering occurs because the individual experiencing it may not be able to articulate what it is about the situation that provokes suffering and could be addressed to alleviate it.

\section{Dignity}

Judgements about an individual's moral character may be made in terms of how well a person copes with pain, or perhaps, how dignified the person is. As Pullman (2002) says: "How one responds in the face of pain and suffering is often thought to contribute to one's sense of dignity. Far from robbing one of dignity it could be argued that the desire to live in spite of pain and suffering expresses and enhances dignity" (Pullman, 2002, p. 75). He goes on to suggest that preconceptions about these concepts and the relationships between them are often not examined, drawing attention in particular to consideration of dignity. Dignity, as invoked in the Preamble to the United Nations Universal Declaration of Human Rights, is an inherent quality of being human, a "universal and unalienable moral 
quality" that can be neither earned nor taken away. Dignity can be diminished, however, in the context of personal agency or circumstance, such as when usually private body parts are exposed to public view against the individual's will: loss of dignity is often associated with losing autonomy and choice. Pullman proposes that because the capacity for choice may be affected by pain and suffering, individuals can feel they have lost their dignity, and it is the sense of obligation to ensure dignity is preserved that serves to motivate people to alleviate pain and suffering (Pullman, 2002). Therefore, a clinician may respond to a person who reports pain out of a desire to restore dignity, particularly if a clinician associates pain as inevitably associated with suffering, and thus loss of autonomy and choice. Unfortunately, this assumption may limit the patient's choices when a clinician holds strong beliefs about the need to alleviate suffering by reducing or eliminating pain in all cases because he or she believes that having pain inevitably means experiencing suffering; fails to ask about the person's primary concern, or is unaware of treatments that address distress and disability.

\section{Distress}

In many situations suffering is associated with expressions of distress. Distress is an emotional state consisting of symptoms of both depression and anxiety, and at times may include somatic symptoms such as headache, or chest pain (Drapeau, Marchand, \& Beaulieu-Prévost, 2011, p. 123).

Distress has been examined in considerable detail in the scientific literature on pain. Despite lack of clarity with both definition and measurement, distress mediates the effect of pain on function (Wegener, Castillo, Haythornthwaite, MacKenzie, \& Bosse, 2011), both pain and emotional distress are mediated by sleep quality and self-efficacy (Miro, Martinez, Sanchez, Prados, \& Medina, 2011), distress is associated with catastrophising (Block \& Brock, 2008), and poorer prognosis, especially in acute or sub-acute musculoskeletal pain (Cai, Pua, \& Kian, 2008; Car \& Sheikh, 2004; Carr \& Goudas, 1999; 
Cedraschi \& Allaz, 2005). Distress is associated with greater treatment-seeking (Epker \& Gatchel, 2000; Macfarlane, Jones, \& Hannaford, 2006), and medication use (McCracken, Hoskins, \& Eccleston, 2006; Mercadante, Villari, Ferrera, \& Arcuri, 2004; Waddell, Bircher, Finlayson, \& Main, 1984). Distress is expressed interpersonally through verbal report or behaviour and appears to be readily identified by observers, particularly those reporting high levels of empathy (Goubert et al., 2005).

Recent neuroscientific research has found that an observer seeing another individual's facial expression while experiencing pain activates neural networks associated with processing pain in his or her own brain (Goubert et al., 2005; Watson \& Greenberg, 2009). At first glance, this would seem to promote and support attempts to reduce distress in the other person, and indeed this regularly happens when, for example, a parent hears his or her child cry. Estimates of pain in another, however, is often inaccurate (Goubert et al., 2005) and there is some research suggesting that individuals may underestimate another's pain as a way to cope with their own distress, or when exposed to pain in others (e.g. those working in Emergency Departments, or partners of people with chronic pain) (Cano, Miller, \& Loree, 2009; Han et al., 2009). More relevant to my argument is the finding that people who report high levels of pain-related catastrophising also infer elevated levels of pain in other people, and attend more to pain behaviour in those experiencing pain (Sullivan et al., 2006). It is thought that catastrophising consists of helplessness, magnification and rumination (Sullivan, Bishop, \& Pivik, 1995) and that those with high levels of helplessness might be cognitively biased towards behaviour they might show if they were in the same situation.

There is a strong assumption by many that people who seek treatment for chronic pain should continue their search until a cure or relief is obtained. This is complicated by the lack of ready acceptance in the community that chronic pain is not the same as acute pain. Healthcare ethics may also contribute to the difficulty some treatment providers have in 
accepting that there are times when it may be necessary to "be cruel to be kind" (Hamlet Act 3 , Scene 4, 178). By this I mean that there are times when clinicians may decline to provide treatment to an individual because although it may help in the short-term, longterm considerations must be kept in mind. Examples of this include when a doctor refuses to prescribe benzodiazepines to a person with high distress associated with low back pain, or chooses not to proceed with further imaging investigations where management will not change, despite being asked by the patient to do so.

For those individuals who feel helpless when observing another person showing distress associated with pain, one response may be to offer help to remove the source of distress (that is, reduce the pain) as a means of reducing personal feelings of distress or helplessness. In clinicians, this strategy may lead to ongoing pursuit of pain reduction as a goal especially when the patient is highly distressed. This may occur in part because this is considered an obligatory aspect of medical treatment, in part because it reduces the sense of helplessness in the clinician, and in part because some clinicians may believe it is inconceivable to live well (and undistressed) while at the same time having persistent pain. Finding a clinician who takes their pain seriously and appears eager to find a way to reduce pain intensity may hold immense appeal for a patient who is distressed by his or her pain and functional limitations, and who has no way to determine whether pain is acute or chronic. It must seem very counterintuitive that this same clinician, if pain reduction is not obtained, may paradoxically maintain distress and disability by continuing to pursue this approach. It is possible that one characteristic of clinicians who continue to look for pain reduction or "cure" is their own level of catastrophising and their heightened empathy for people experiencing pain. This is undoubtedly an ethical dilemma for clinicians working with those who have chronic pain. 


\section{Treatment seeking and pain}

New Zealanders, Australians and people from most developed countries today have, however, largely embraced the expectation that pain should be cured, or at least reduced. Medical practitioners and patients express frustration when their expectation for pain relief cannot be met, yet it is only just over one century since health care professionals could access effective anaesthetics. Before the latter part of the nineteenth century, pain was a normal part of life, and a combination of religious and cultural attitudes contributed to the notion that experiencing pain was indispensable for developing moral character. Pain and suffering was seen as a means to "strengthen the religious person's bond with God and other persons" (Glucklich, 1999). Thernstrom (2010) traces the history of attitudes towards pain relief and argues that the meaning of pain in Western culture has changed irrevocably since surgical anaesthesia was introduced. She concludes that society has reframed pain from being primarily a religious or spiritual metaphor to simply being a biological by-product of disease (p. 284). This move creates an environment in which pain is viewed as a state that both can, and should, be eliminated; culminating in the International Association for the Study of Pain's "Declaration of Montreal" in which signatories assert that access to pain management is a fundamental human right. Importantly, pain management is not the same as pain abolition. Experiencing pain is an indispensable protective feature of human existence, and there are circumstances in which pain cannot be removed or reduced. For most people, experiencing pain initiates a search for ways to relieve it. Before discussing the situation for those in whom pain cannot be removed, I will explore influences on seeking treatment.

While some women cope with the severe pain of labour without analgesia, many people look for pain relief for pain of varying degrees of intensity. Treatment seeking refers to the process in which an individual pursues help for his or her health problem. It has been extensively investigated in the pain research literature because this behaviour is a central 
issue in health care delivery, in part because of its relationship to cost and policies of health care delivery. The relationship between experiencing pain and seeking healthcare, however, is not straightforward. Illness or deviation from a state of health is mostly a subjective state, and the process of seeking treatment is highly influenced by psychosocial factors, while individuals seek relief both within or external to conventional medical and health facilities making it difficult to estimate use (Rabin \& Schach, 1975). In acute pain, treatment seeking is associated with factors such as the degree of disability or interference and general health status (Cote, Cassidy, \& Carroll, 2001); socialisation toward accessing healthcare (Cardol et al., 2006); level of distress (Feuerstein, Harrington, Lopez, \& Haufler, 2006); presence of previous healthcare consultations for similar problems (Grooten, 2007); socioeconomic factors such as ready access to healthcare (Paananen et al., 2011); and in women, the presence of workplace stress (Skillgate, Vingard, Josephson, Theorell, \& Alfredsson, 2007). Interestingly, pain intensity may play a lesser role than often assumed with disability (Ferreira et al., 2010a), and factors related to past learning or socialisation (Guite, Logan, McCue, Sherry, \& Rose, 2009), as well as intrapersonal factors such as beliefs and cognitive style playing a more influential role (Jensen, Haahr, Frost, \& Andersen, 2012). Greater frequency of healthcare consultation for pain is associated with psychosocial factors such as health anxiety, depression, fewer coping resources and comorbid health conditions (Cote et al., 2001; Ferreira et al., 2010a; Jensen et al., 2012; Johnson Wright, Afari, \& Zautra, 2009). It is also noteworthy that different types of pain may be associated with different treatment seeking behaviour. For example, in a study by Oliveira, Leite, and Rocha-Filho (2011), the pain complaints of a total of 200 consecutive patients attending their family doctors were recorded. Participants were asked about headaches during the past 12 months, and those reporting at least one episode of headache completed a semi-structured interview. This study found that $52 \%$ of patients had experienced headache, but only $10 \%$ of the total group sought medical 
treatment for this. Those with tension-type headache requested treatment for their pain less often than those with migraine, and these individuals also reported their pain intensity was "mild", yet there was no difference between the groups in terms of the impact on their daily lives. People with migraine more readily accessed healthcare, while those with tension-type headache reported that analgesia alleviated their pain and consequently did not feel the need to ask for medical assistance.

\section{Catastrophising}

I have referred to catastrophising, or the tendency to think the worst, many times within this discussion of chronic pain. Catastrophising is thought to be a key cognitive bias implicated in numerous negative outcomes involving both negative interpretations of both distinctly negative events, as well as neutral or ambiguous cues (Roelofs, Peters, $\&$ Vlaeyen, 2002). Outcomes particularly influenced by an individual's tendency to catastrophise include pain intensity, distress and disability (Keefe et al., 2004), and it has also been shown that individuals may experience reduced threshold and tolerance for pain when they are directed to attend to negative emotions associated with their pain. This suggests that catastrophising may bias individuals towards processing the most disturbing aspects of their pain experience (Michael \& Burns, 2004). Individuals who tend to catastrophise are at greater risk for experiencing suicidal ideation (Edwards, Smith, Kudel, \& Haythornthwaite, 2006), demonstrating greater pro-inflammatory responses to noxious stimuli (Edwards et al., 2008), and poorer response to rehabilitation efforts and even to biomedical treatments for pain such as total knee arthroplasty (Roth, Tripp, Harrison, Sullivan, \& Carson, 2007). Catastrophising exerts an influence over multiple systems in an individual with pain.

\section{Pain intensity or psychosocial factors?}

Many psychological aspects of pain and treatment seeking have been, and still are, the subject of research, including factors associated with mental defeat (Tang et al., 2010); 
female gender (Mailis-Cagnon et al., 2007); lack of social support (Skomo, Desselle, \& Berdine, 2006); high levels of disability (Ferreira et al., 2010b); attachment style (Ciechanowski, Sullivan, Jensen, Romano, \& Summers, 2003); number of concurrent symptoms (Grooten, 2007); high pain intensity (Jacob, Zeev, \& Epstein, 2003); somatization and health anxiety (Jensen et al., 2012); presence of neuropathic pain (McDermott, Toelle, Rowbotham, Schaefer, \& Dukes, 2006); coping styles (Skillgate et al., 2007); socioeconomic status, ethnicity, age and depressive symptoms (Andersson, Ejlertsson, Leden, \& Schersten, 1999).

Reviewing the various factors differentiating between those who have chronic pain and those without chronic pain some key dimensions are particularly relevant to healthcare use, such as attitudes and beliefs (e.g. catastrophising), behaviours (e.g. avoidance), and critically, how readily they access health care generally (Tan, Jensen, Robinson-Whelen, Thornby, \& Monga, 2001; Turk \& Rudy, 1990, 1991; Turk, Rudy, \& Sorkin, 1993). These findings support the argument that pain intensity may be less influential than psychosocial variables in determining the ways humans respond to their experience of pain. Social, cultural factors and personal learning history influence attitudes, beliefs and behaviours in an individual and can potentially be modified. This provides clinicians and policy-makers with a range of options for reducing the risk of long-term disability associated with the experience of pain (Keefe et al., 2004; Turk et al., 2011).

\section{Characteristics of those who do not seek treatment}

People who have pain but do not seek treatment are less well-studied; however it is clear that this group represents a fairly large minority. Epidemiological studies such as the MUSIC-Norrtalje study (Mortimer, Ahlberg, \& Group, 2003) and Walker, Muller and Grant's study in Australia (2004) have found up to 55\% of people with low back pain do not seek treatment but manage their symptoms themselves, while Dominick et al. (2011) found that a third of people reporting chronic pain in a national health survey of New Zealanders did 
not use any treatment. Despite these findings, it is difficult to identify many studies that specifically recruit and study people who have persistent pain but are not seeking treatment. Most studies looking at coping strategies in chronic pain have used participants from those seeking treatment (Clarke \& Iphofen, 2007; Evers, Kraaimaat, Geene, Jacobs, \& Bijlsma, 2003; Evers et al., 2001). This may reflect difficulty locating participants for studies of people who identify as coping well, but may also reflect the prevalent model of health care which is oriented towards dysfunction, ill health and identifying problems. By failing to include participants who cope well with pain, however, researchers and clinicians risk making decisions about pain coping from nonrepresentative samples. As a result, they may inadvertently exclude strategies that work effectively in the context of real life, or they may emphasise strategies that fail to translate well into people's daily lives.

There are several ethical dilemmas for clinicians involved in pain management to consider when treating people with chronic pain. I have suggested that the concept of suffering may be inappropriately conflated with pain such that any individual who reports that they have pain, and particularly an individual demonstrating high levels of distress, may be offered treatments even if these have limited effect. I have also suggested that factors associated with seeking treatment reflect psychosocial vulnerabilities rather than pain intensity. Individuals who are prone to catastrophising may be at particularly risk of being directed towards pain reduction interventions that may do little to address the underlying psychological tendency to misinterpret or over-interpret the experience of pain. At the same time, while there has been considerable focus on understanding those who seek treatment for their pain, I argue there has been limited attention paid to examining the characteristics of those who do not seek treatment, thus restricting knowledge of effective coping strategies. By studying people who manage their pain independently there is an 
opportunity to learn ways to enhance individuals' capacity to cope with pain, and to learn about what works in daily life.

\section{Managing pain}

Biomedical management of pain includes the use of pharmaceuticals, anaesthetic procedures, and surgery, with the aim primarily to diagnose and cure the underlying cause, or to reduce pain intensity when cure is not possible. Inherent in this model of pain management is the assumption that once the pain is reduced or eliminated, the person's life continues as before. Despite advances in biomedical management options, however, a substantial group of people fail to respond, and go on to develop chronic pain with associated disability, and some individuals remain disabled despite having achieved effective pain reduction (Turk et al., 2011). The most well-established management option for these people is interdisciplinary pain management with a cognitive-behavioural selfmanagement approach (Day et al., 2012).

Programmes based on cognitive-behavioural self-management have existed since the mid-1970's, and although it is unclear which elements are necessary and sufficient to produce change, programmes typically include psycho-education on pain mechanisms; physical reactivation; development of active coping strategies; and goal-setting (Day et al., 2012; Jeffe, Butler, Stark, \& Kane, 2011; Watson, 2000). The general theoretical basis is cognitive behavioural, designed to teach people how cognitive, affective and physiological factors influence their experience of pain, so they can increase their sense of self efficacy for managing it. Combined with the psycho-educational components, participants in these programmes learn skills to help them change the way they live with their pain (Keefe, Dunsmore, \& Burnett, 1992). Pain intensity is not the main focus of these programmes, instead disability, or the effects of pain on function become the target. Active coping strategies are encouraged, such as paced activity, effective relaxation, maintaining a consistent exercise quota, and developing and achieving personal goals. The outcomes of 
these programmes are measured using questionnaires to evaluate the range and use of various coping strategies (Turk et al., 2003).

\section{Coping}

In Chapter One, I defined coping strategies as the strategies people use to remain engaged in doing what is important, while coping itself refers to the outcome of using these strategies. In a sense, every effort to keep doing what is important can be considered coping, but clinicians and researchers may judge these efforts as "good coping" or "poor coping". The judgement may be made depending on how much the individual using them experiences distress and disability. This position is arguable, as I will show. During chronic pain management programmes, cognitive behavioural-oriented clinicians introduce patients to a range of coping strategies. To review the entire range of strategies is beyond the scope of this thesis, however there are some themes and organising theoretical models that are pertinent to consider.

In the gate control theory of pain, Melzack and Wall (1965) proposed the concept of pain modulation through peripheral and central mechanisms, opening the way to explaining how psychological processes shape the experience of pain. In a real sense, this model enabled the biopsychosocial model (Engel, 1977) to be applied to pain, and enabled clinicians to discover approaches to reduce disability and distress where pain cure or relief could not be achieved.

\section{Behavioural approaches}

Coping strategies can be broadly divided into cognitive or behavioural. As outlined earlier in this chapter, behavioural approaches to managing chronic pain emerged from the work of people like Fordyce et al. (1968) who applied behaviour therapy principles, in particular operant conditioning, to modify unhelpful pain behaviours and increase well behaviour for people with chronic pain. The behavioural approach involves carrying out behavioural 
analysis of antecedents and consequences of behaviours and applying contingency management to alter the frequency of overt behaviour. This approach incorporated the social context, including family responses and extended to the community and even legislative influences on disability; however it became less popular as cognitive approaches emerged in the mid to late 1970's. It is routinely employed today in functional restoration/activation approaches in which individuals incrementally increase activity levels by following a quota rather than cardiovascular or pain contingent approaches (Stanos, 2012; Turk et al., 2011), and as part of biofeedback to modulate physiological responses to pain (Flor \& Turk, 2011).

Along with operant conditioning, clinicians using respondent conditioning have also contributed towards approaches for mitigating disability associated with chronic pain. Rather than influencing contingencies, in the respondent conditioning paradigm pain behaviours are thought to develop via classical conditioning. This approach underpins the pain-related fear and avoidance model, in which graded exposure to feared and avoided stimuli counters behavioural avoidance (Vlaeyen, Kole-Snijders, Boeren, et al., 1995).

\section{Cognitive behavioural approaches}

Behavioural approaches are rarely if ever, applied alone. Research shows behavioural approaches affect not only behaviour, but also influence cognitions and emotions, particularly catastrophising and fear (de Jong et al., 2005; Vlaeyen et al., 2002). Chronic pain management programmes incorporate cognitive approaches alongside behaviour change strategies based on the assumption that the way individuals interpret the world influences both emotional and behavioural responses (Turk, 2002). Consequently, beliefs, appraisals, coping skills repertoire, behaviours, and/or events in the social environment are targets for therapy. 
Fundamental strategies introduced within a cognitive behavioural approach include the following:

- Providing accurate information about chronic pain;

- Reappraising pain in a more positive and realistic way;

- Introducing effective problem-solving skills to enable individuals to consider how they might manage a situation rather than avoid;

- Developing skills to engage successfully in those situations previously avoided by using effective communication techniques, stress management skills such as relaxation, and forming appropriate goals and contingencies when attempting such goals (Day et al., 2012; Flor \& Turk, 2011).

\section{Classification}

There are numerous models for classifying and conceptualising coping. A chapter by Chronister and Chan (2007) describes a hierarchical conceptual framework developed by Krohne (1996) for organising the various models and theories, moving from a macroanalytic conceptual level to situation-specific micro-analytic coping categories. Chronister and Chan (2007) point out that analysis at the micro-analytic level can examine the transactional and temporal nature of the coping process in more detail while macroanalytic analysis can generate theoretical explanations for how humans self-regulate and manage stressors.

Chronister and Chan (2007) suggest that approach and avoidance are two main concepts central to all the various macro-analytic models. Fundamentally, it appears that humans either approach and move towards a stressor, or alternatively avoid or move away from a stressor. While there are many variations from a range of theoretical schools on these concepts within the macro-analytic categories, critics of research from this perspective argue that macro-analytic models fail to discriminate between traits, have limited 
explanatory power (particularly if individuals use both approach and avoidance strategies), and do not explain how individuals choose different strategies in different contexts therefore cannot predict variability in coping behaviour (for example (Krohne, 1996; Lazarus \& Folkman, 1984b).

Relevant to pain as a stressor, Krohne's model of coping which is a macro-analytic theory incorporates concepts of vigilance, or an increased focus on threatening information, and cognitive avoidance, or turning away from threatening cues, particularly in situations where aversive stimuli are presented in an ambiguous way (Krohne, 1996). Krohne (1996) considered that individuals vary in the degree to which they can tolerate both uncertainty and emotional arousal as a matter of disposition, with individuals who find it difficult to tolerate uncertainty tending to be highly vigilant, and those who find it difficult to tolerate emotional arousal tending to use cognitive avoidance. Krohne's model suggests that people who tend to be vigilant preferentially attend to threat-relevant stimuli, preferring to be faced with situations in which they experience emotional arousal rather than being faced with an unpleasant surprise. Conversely, individuals who are less comfortable with emotional arousal employ cognitive avoidance strategies and ignore threatening cues because they are less concerned about situations of ambiguity. In terms of coping with pain, individuals with, for example, a lower tolerance for ambiguity but high tolerance to emotional arousal may preferentially seek confirmation of a diagnosis they can understand, rather than managing the ambiguity of not having a clear diagnosis. It may also lead to these individuals being particularly concerned about and over-interpreting ambiguous sensations, identifying these as threatening, thus indicating the need to obtain treatment. Individuals with this set of coping tendencies who have chronic pain with no clearly identified cause may be particularly vulnerable in this regard. Krohne's model has not been explored recently within the pain literature, but aspects of it have been 
investigated such as preferential processing of pain-relevant stimuli (Asmundson $\&$ Hadjistavropoulos, 2007; Vancleef et al., 2009).

Probably the most commonly reported broad classification divides coping strategies into adaptive and maladaptive strategies. Jensen, Turner, Romano, and Karoly (1991) reviewed the literature and identified a number of the classifications, finding one main theory-based approach: problem-focused coping and emotion-focused coping, drawn from ; with the next most common classification being active and passive coping (derived from factor analysis rather than theory) (Brown \& Nicassio, 1987; Lazarus \& Folkman, 1984b).

Commentators have criticised these classifications, pointing out that "what one investigator views as a behavioural coping effort (e.g. resting in bed, taking medications), another investigator is likely to view as an outcome of coping" (Keefe, Salley, \& Lefebvre, 1992, .p 132) and also arguing that the lack of longitudinal studies to examine the relationships between coping and emotional distress mean it is difficult to determine the temporal relationships between the two variables (Keefe, Salley, \& Lefebvre, 1992, .p 132).

Similarly, outcome studies show contradictory findings on the use of coping strategies: activity pacing is a strategy often recommended in pain management programmes but has been associated with both positive (Karsdorp \& Vlaeyen, 2009) and negative outcomes (McCracken \& Samuel, 2007b). Persistence or endurance has been associated with both poorer and greater levels of physical functioning (Andrews, Strong, \& Meredith, 2012). A study examining psychological flexibility and traditional pain management strategies in relation to patient function found that traditional pain management strategies did not account for variance in any one of eight measures of functioning (McCracken \& Vowles, 2007).

Day and colleagues suggest that it may not be the techniques or strategies employed in programmes for pain management that influence outcomes, and instead attribute change 
to certain factors common to psychotherapeutic interventions such as having an effective therapeutic alliance (Day et al., 2012).

\section{Coping theory}

Chronic pain represents a significant challenge for individuals to manage. Coping strategies are intended to promote positive emotion and self-image; a sense of mastery and competence; have supportive relationships with family and friends, and prepare for the future (Moos \& Holahan, 2007). Strategies used to achieve these goals are called adaptive tasks, and de Ridder and Schreurs (2001) argue that this concept implies having adopted a stress coping framework.

The model of stress developed by Lazarus and Folkman (1984a) has been studied extensively. It forms an organising framework for understanding the process of dealing with chronic pain, and fits comfortably within the cognitive behavioural tradition. In brief, this model considers that individuals appraise an event in the first instance as threatening, challenging or neutral (primary appraisal). In response to primary appraisal, secondary appraisal involves determining whether or not he or she has the necessary resources to cope with the situation. If the individual does not believe he or she has the resources to manage effectively, this is experienced as stress, while if the individual believes he or she has enough resources to cope, coping strategies are enacted and the outcome of this effort is in turn appraised as part of a feedback loop. The coping strategies themselves serve to manage or alter the stressor itself (problem-focused coping), or regulate emotional responses to the situation (emotion-focused coping). Interestingly, in 1980 when Folkman and Lazarus (1980) published a study of coping in people living in the community without identified pathology, they pointed out that coping is both multidimensional and a shifting process in which individuals may need to use different strategies at different times as a situation unfolds. They used this argument to support their view that coping is not a trait, preferring their own view that coping is a series of 
transactions between the individual and his or her environment, mediated by appraisals and coping. This observation can, however, also support the argument that assessments of coping must take into account changing circumstances and strategies over time rather than viewing coping as a static set of strategies measured at one time. Coping assessment measures, however, may not adequately accommodate this need. Individuals experiencing chronic pain use both problem-focused and emotion-focused coping, although problem-focused coping is typically associated with lower levels of distress and disability, while emotion-focused coping is associated with appraising pain as a threat and associated with greater distress (for example Dysvik, Natvig, Eikeland, \& Lindstrom, 2005). These findings, however, are not consistent.

The second classification approach used in chronic pain coping research draws from the Vanderbilt Pain Management Inventory (Brown \& Nicassio, 1987). Brown and Nicassio argued "irrespective of their type, coping strategies may be classified as adaptive or maladaptive based on their relationship to indices of pain and psychosocial functioning" (p. 54) and proposed that active or adaptive coping strategies aim to control pain or to support individuals to function despite pain while passive or maladaptive coping strategies involve relinquishing control of pain to others, or allowing areas of life to be adversely affected by pain. The major difference between Brown and Nicassio's passive and active classifications and the problem-focused and emotion-focused classifications are that the former did not incorporate an a priori theoretical model, but used factor analytic techniques to generate the two scales.

General models of coping with illness have also been studied in relation to coping with chronic pain. Amongst these models are the Common Sense Self-regulatory Model developed by (Leventhal et al., 1992), and the five factor Integrative Conceptual Framework by Moos and Holahan (2007). 
The Common Sense Model was developed in the early 1980's by Leventhal and colleagues to explain how lay models of health influence treatment adherence through illness representations (Leventhal et al., 1992). The model incorporates illness representations held by individuals (an appraisal of the meaning of the health situation), the emotions experienced in response to the illness representation, coping strategies and finally, appraisal of the outcomes. Outcome appraisals feed back to the "situational stimulus", and the individual reappraises the situation in terms of what it signifies to him or her. The Common Sense Model identifies how individuals process information about their health, integrate this information with their own views then use this personalised model of their health to guide what they do.

The model has been elaborated and refined since its introduction and a meta-analysis found that the processes articulated in the model apply across a wide range of health conditions (Hagger \& Orbell, 2003), but while it can be used to identify processes involved in appraisal, it appears less well-developed in terms of how individuals select appropriate coping strategies.

Within the Common Sense Model, there are two groups of coping strategies: (1) coping strategies to deal with the illness itself, and (2) coping strategies used to deal with the emotional reaction to the illness. Illness representation (cognitions) filters and interprets information about an illness and influence the choice of coping strategy related to the perceived challenge it represents. Identified coping strategies include avoidance/denial, cognitive reappraisal, expressing emotions, problem-focused coping and seeking social support. Findings from empirical research show that individuals with strong illness identity and high cure/control beliefs use active coping, seeking social support and behavioural disengagement (for example, Moss-Morris, Petrie, and Weinman (1996)). Those with a strong illness identity and low cure/control beliefs are more likely to use avoidance and denial as coping strategies (Hagger \& Orbell, 2003). 
The Common Sense Model shows how illness representations develop by integrating community and family views, information from health professionals, and the individual's personal experience. The importance of social factors in the experience of pain has been highlighted by the impact of a media campaign on both reporting back pain, and the duration of back pain claims in Australia (Buchbinder \& Jolley, 2005; Buchbinder, Jolley, \& Wyatt, 2001). In this study a media campaign giving strong messages about keeping active despite back pain was shown to reduce the number of claims for back pain even though the original intention was simply to reduce the duration of claims. Furthermore, the effect of the campaign was sustained for more than three years. In addition to such community-focused interventions, the influence of both clinicians and family members on treatment seeking and both beliefs and behaviours associated with pain is wellestablished (Cano et al., 2009; Cardol et al., 2006; Giardino, Jensen, Turner, Ehde, \& Cardenas, 2003; Main et al., 2010; Paananen et al., 2011).

Moos and Holahan (2007) developed an integrative framework for coping with chronic illness and disease. This model consists of five 'panels' of factors thought to influence the choice of coping strategies. Like the Common Sense Model, the integrative framework is a systems model with health-related outcomes influencing the remaining four factors of (1) personal resources, (2) health-related factors, (3) social and physical context, (4) health condition appraisal, and (5) adaptive tasks. The choice of coping skills is identified as the fifth 'panel', with health outcomes as the sixth.

This model conceptualises adaptive tasks as relating to the health condition and management, as well as a further set of tasks related to more general life challenges. Coping strategies are classified according to two dimensions: approach and avoidance, and cognitive or behavioural. Moos and Holahan (2007) therefore identify four categories of coping: (1) cognitive approach coping involving analysing, positive reappraisal and looking for meaning; (2) behavioural approach coping including looking for guidance and 
support, problem solving action; (3) cognitive avoidance coping such as denial, resignation, not thinking about the situation; and (4) behavioural avoidance coping involving looking for alternative rewards and expressing emotions. Moos and Holahan (2007) point out that an individual may use a selection of these strategies, and different ways of coping at different times over the course of an illness.

Interestingly, neither of these two models incorporates the current life direction of individuals, nor ways of coping that support goals outside of illness. This limits the explanatory power of both models in that the values individuals place on their health and the choice of coping approach may be influenced by competing goals or values such as fulfilling the parental role, or remaining engaged in sporting, employment, social or artistic occupations.

\section{Strategies included in chronic pain management programmes}

While chronic pain management programmes are based on broadly cognitive behavioural principles, the specific components of such programmes vary greatly (Day et al., 2012). The overarching aims of these programmes usually do not directly target pain intensity, but primarily target disability and distress. Although each programme differs depending on individual clinicians and the disciplines of the professionals involved in them, their aims are typically to:

- Reconceptualise the pain from uncontrollable to manageable

- Foster optimism and combat demoralisation

- Promote feelings of success, self-control, and self-efficacy

- Encourage patients to attribute success to their own role

- Educate in the use of specific skills such as pacing, relaxation, and problem solving

- Emphasise active patient participation and responsibility (Turk et al., 2011, p. 2331) 
Common components in an interdisciplinary pain management programme include physical reactivation, exercise therapy, cognitive restructuring with an emphasis on promoting self-management, self-efficacy, resourcefulness, and activity versus passivity, reactivity, dependency, and hopelessness; behavioural treatment (for example, relaxation, work to exercise quota vs pain), vocational rehabilitation, where indicated and drug management as needed (preferably with reduction of opioid treatment) (Turk et al., 2011, p. 2331).

Drawing from the coping models above, programmes endorsing these strategies aim to enhance the range of coping options available to an individual, promoting approach strategies rather than avoidance, incorporating new information to modify cognitive appraisal, therefore increasing participants self-efficacy to engage in valued activities. Participants are expected to learn these skills and apply them in the context of their own lives, however specific bridging from coping skills content to personal context may not be not explicit, with the exception of plans for return to work.

\section{Limitations in knowledge of coping}

While there have been numerous attempts to classify coping strategies differently according to an underlying theory, there are problems with ascertaining how individuals use coping approaches in daily life.

A major limitation to a deep understanding of coping is the difficulty of obtaining data without recourse to self-report. Additionally, coping questionnaires typically ask individuals to recall the strategies used over the course of a period of time, or to rate the frequency with which they use them. Recall bias can influence these measures (Stone et al., 2004), and additionally, cognitive changes such as the effect of information on primary appraisals are not incorporated into common measures of coping such as the Chronic Pain Coping Inventory. This may be because these techniques are not conceptualised as 
strategies per se by either researchers or people with chronic pain (Jensen, Turner, Romano, \& Strom, 1995). They do, however, influence behavioural outcomes such as distress and disability (Chiros \& O'Brien, 2011). While daily diary or ecological momentary assessment methods provide a way to minimise recall bias, they can be time-consuming and may intrude on participant's usual daily routine (Schwarz, 2007), and there are cost implications when considering electronic devices to collect data. These methods also do not address changes to primary appraisals.

Coping questionnaires also do not include contextual factors such as the activities the individual is doing, the intention behind employing a coping strategy, or the individual's goals, both short and long-term (Lauwerier et al., 2012). The majority of coping studies are carried out on patient populations, with the original items for questionnaires generated from researcher or clinician ideas rather than derived from people who live well despite pain (Hadjistavropoulos, MacLeod, \& Asmundson, 1999). This may mean strategies employed by individuals are not included, or the emphasis or frequency with which strategies are used by non-treatment seeking individuals is not known.

Coping strategies used by an individual may change over time (Heim, Valach, \& Schaffner, 1997). When pain is first experienced, strategies appropriate to its management may be used such as rest and muscular guarding, while later on these same strategies are less helpful and may be replaced by gradually increasing activity levels and using relaxation techniques to reduce muscle tension. Unfortunately coping questionnaires such as the Chronic Pain Coping Inventory (Jensen et al., 1995) ask respondents to consider how often they use the strategies over a fairly short period of time such as one week or one month, and do not establish why each strategy is used. Finally, very few longitudinal studies have examined the use of coping strategies over years, or over the course of a lifetime, thus it is difficult to determine whether different coping strategies are used at different times in the course of living with chronic pain. 
Outcome measurement tools, such as the IMMPACT (Initiative on Methods, Measurement \& Pain Assessment in Clinical Trials)(Turk et al., 2006) measure patient-reported outcomes, but fail to measure which coping strategies or approaches are used to achieve these outcomes. As a result of these limitations, while there is a large body of research on coping strategies and various associations with disability and distress, there are qualifications on how well these findings reflect the real-world experience of individuals living with chronic pain.

\section{Moving towards models of ability and resilience in pain}

Disability can be defined as "any restriction or lack (resulting from an impairment) of ability to perform an activity in the manner or within the range considered normal for a human being" (WHO, 1980). The social model of disablement suggests that disability arises from the limitations of a society that categorises who is normal and who is not, and points out that the WHO model is largely a deficits-based model. Individuals with pain do, however, experience constraints on activities they value (Raheim \& Haland, 2006), thus while a social model may hold true in terms of the ways people with chronic pain are viewed within their community, at least some of their experience arises from their own decisions to restrict activity.

The deficits model or approach to health has been challenged over the past 20 years with the advent of concepts such as resilience, acceptance and recovery. These concepts focus on enhancing existing strengths and values, living despite having pain, and reducing the amount of energy spent on controlling pain and associated cognitions and emotions (McCracken \& Eccleston, 2005; Nicholas \& Asghari, 2007; Seligman \& Csikszentmihalyi, 2001).

A paradigm shift towards working with client strengths has been evident in the disability literature since the mid-1970's, but much more evident in mainstream psychology since 
Seligman's paper on positive psychology and health in 2000 (Resnick \& Rosenheck, 2006; Seligman \& Csikszentmihalyi, 2001; Seligman, Rashid, \& Parks, 2006; Vaillant, 2000). Seligman's proposition was that psychology, in focusing on treating mental illness, had lost its ability to identify ways to prevent ill health. He introduced a concept of positive psychology that is now providing an impetus towards focusing on human resourcefulness and strength. Subjective well-being, optimism, happiness, and self-determination have provided insights into the ways these characteristics can be fostered in people facing adversity (Britt, Dickinson, Moore, Castro, \& Adler, 2007; Dunn \& Dougherty, 2005; Zautra, Hamilton, \& Yocum, 2000; Zautra, Arewasikporn, \& Davis, 2010; Zautra, Johnson, \& Davis, 2005).

The independent living model introduced in the mid-1970's proposes the idea that the problem of disability is a societal one and argues that disability can be seen as a natural and not uncommon experience in life, rather than a tragedy (DeJong, 1979). As a response to this stance, the independent living model encourages people with disabilities to make health decisions in partnership with health providers, rather than health providers making decisions on their behalf. The health provider's role is to provide opportunities for people with disabilities to learn about choices relevant to their lives, and the consequences of those choices. The independent living model is used in approaches to recovery from brain injury, spinal injury and in managing chronic illness (Batavia, DeJong, \& McKnew, 1991; Fluharty, Sellon, \& Glassman, 1994; Mattson-Prince, 1997).

The analogue in mental health, the recovery model, has made a significant impact on the roles and activities of health professionals working with people experiencing long term mental health problems, and drug and alcohol dependence (Lunt, 2004; Oades et al., 2005; Ramon, Healy, \& Renouf, 2007; Reisner, 2005; Resnick \& Rosenheck, 2006; Smith \& Bartholomew, 2006). When using this model, health professionals encourage clients/patients to identify their strengths and preferences, and work together to achieve 
life goals. While this has occasionally led to conflict between clinicians and clients because the values and goals of each party differ, the recovery model emphasises the rights of the person to live a life according to his or her preferences, with the health professional's contribution being to provide information about choices (Lunt, 2004).

The discourse about independent living and disability rights of people with pain seems to have had limited impact in the pain literature. Although research into the patient experience exists (primarily using a phenomenological approach), this has not translated into a strong patient-led disability rights or independent living movement with a high public profile within New Zealand (Maly \& Krupa, 2007; Skuladottir \& Halldorsdottir, 2011; Slade et al., 2009). Individuals with pain may seek social support from groups such as Endometriosis New Zealand or Arthritis New Zealand, but there are few calls for greater public awareness and acceptance of chronic pain and even fewer calls for equal status within the community as has been the case for other chronic health conditions such as spinal cord injury. Instead, the public discourse has been on ways to reduce pain, and return to normal life, with normal being equated to being pain-free.

Clinicians have developed treatment approaches based on their knowledge of the research, which has, as noted above, been largely based on findings from people who seek help to cope with pain. The voices of those who cope successfully with their pain, and live well without seeking further pain reduction are rarely heard. This leaves a potential gap between what clinicians believe people with chronic pain should do, and the ways people who live well with their pain may actually manage in daily life.

In an earlier section of this chapter, I described three models providing theoretical explanations for differences in pain-related disability, and indeed, there are other models in the literature (Main et al., 2008), but explanations for resilience in people with chronic pain are lacking (Karoly \& Ruehlman, 2006). Resilience is an increasingly popular area for research in health and psychology (Atkinson, Martin, \& Rankin, 2009; Seery, 2011; Stewart 
\& Yuen, 2011), with much of the work focusing on aspects of coping style and resource that make resilience possible such as optimism and social support (Smith, Dalen, et al., 2008). Resilience refers to "health despite adversity" and invokes concepts of maintaining life tasks or occupations by returning to a homeostatic condition, by altering the ways an individual goes about achieving those activities, and by remaining undistressed while doing so (Dunkel Schetter \& Dolbier, 2011; Herrman et al., 2011). Little of this research looks at coping with chronic pain (Karoly \& Ruehlman, 2006), and clinicians wishing to use a strengths approach to enhance resilience in patients with chronic pain will find it difficult to base this on a pain-specific theory.

There are some exceptions to this paucity of research into pain-related resilience and coping strategies used by non-treatment-seeking people in the community. Early work by Strong and Large (Strong \& Large, 1995) using focus groups found that people living in the community with low back pain, and who were not seeking treatment, used a range of coping strategies at different times. One particular strategy was to attend to symptoms and vary the strategies employed accordingly depending on context. All the participants considered exercise to be an important way of coping even though this may not reduce pain at the time (p. 377). Participants in this study described changing the way they manage their pain over time as they recognised a shift from acute pain to chronic pain. The authors suggested a shift of this nature requires conscious effort, and reported that participants described "taking charge", although also acknowledging that planning and "trade-offs" informed their decisions (p. 378).

In a second study, this time using construct theory, Large and Strong (Large \& Strong, 1997) found that some people with low back pain viewed themselves as having "no choice but to cope" (p. 247), while others within the same group saw themselves as expressing "mastery". The authors concluded that although the participants valued being a coper, their construals were ambivalent and being pain free was the most desirable state. 
Coping, in this group, was a "necessary evil" but underpinned their sense of self-worth and social persona (p. 251).

More recently, the work carried out by Dr Alex Zautra and colleagues at the Arizona State University. Sturgeon and Zautra (2010) identifies three groups of resiliency outcomes: (1) recovery, referring to the degree to which an individual "bounces back", or returns to normal following stressful events; (2) sustainability, or the extent to which an individual perseveres with goals, activities and actions that promote positivity and self-esteem; and (3) growth which refers to developing a greater recognition of positive attributes and capabilities, and learning that occurs as a result of coping with stressful experiences ( $p$. 106).

Sturgeon and Zautra (2010) distinguish between the resources supporting the resiliency outcomes identified above and the mechanisms through which these resources produce positive outcomes. Resilience mechanisms are thought to moderate the relationship between experiencing pain and maintaining wellbeing (p. 106).

Three important characteristics have been identified as resilience resources in people with chronic pain. Optimism is thought to increase healthy behaviours, reduce unhealthy behaviours and thus improve physical functioning, and is defined as being able to maintain positive expectations for the future (Bargiel-Matusiewicz \& Krzyszkowska, 2009; Ramirez-Maestre, Esteve, \& Lopez, 2012; Smith, Tooley, et al., 2009). Optimism is also thought to support persistence towards personal goals, which in turn provide purpose in life, also thought to contribute to a sense of fulfillment and meaning (Smith, Tooley, et al., 2009). Having a purpose in life provides individuals with forward momentum to achieve goals and influenced recovery times after knee replacement surgery (Smith \& Zautra, 2004). Finally, acceptance is being increasingly studied, and refers to general willingness to experience pain so an individual can remain engaged in what is important to them, rather than avoiding or attempting to control pain (McCracken, 2010; McCracken \& 
Eccleston, 2005, 2006). Acceptance enables individuals to move from focusing on what to avoid towards focusing on what is valued, or gives pleasure and purpose in life.

McCracken's work on acceptance is framed within the Acceptance and Commitment Therapy (ACT) model of psychological flexibility. ACT is based on a functional contextual philosophy, a philosophy that considers behaviour or actions as a function of historic and current circumstances (Fox, 2006). In other words, behaviour can be explained in terms of what it achieves within a specific context. An example of this might be using rest as a strategy for managing pain. While resting limits an individual's involvement in many daily activities it often reduces pain intensity. As long as avoiding pain is the goal, and the individual has no other important goals or values in life, resting is a useful and helpful strategy. It is only when this behaviour is viewed in the context of other important goals or values such as paid employment or sporting activities that resting is seen as less useful, because these are difficult to achieve while lying down.

While these few studies provide some insight into the ways people who do are not usually seen in treatment setting manage their lives and pain on a daily basis, there is much to learn. Two important points must be highlighted: the first is that individuals may not be aware of why they are doing what they do, for example avoid pain but also restrict their involvement in what they value; and the second is that because much of the research into living with chronic pain has focused on those who have difficulty coping, and has often looked at skills used outside the context of personal goals and values, there is limited awareness of existing strengths within individuals that can be supported, and little knowledge of ways clinicians can encourage flexible coping despite pain. Indeed, pursuing pain relief as an exclusive goal may prolong distress and disability (Lauwerier et al., 2012; Van Damme et al., 2008). A pertinent question to ask is why some people with pain, and some clinicians, persist in seeking pain reduction as an end in itself, without considering the cost of doing so, and it is to this I turn next. 


\section{Justifying a theory of how people positively manage chronic pain}

I began this chapter by defining pain and pointing out that pain is an experience that is subjective and influenced by biopsychosocial factors. While acute pain is stressful, it is also adaptive and protective and relatively well-accepted as a phenomenon in the community. Chronic pain, on the other hand, is far more ambiguous, maladaptive and presents a complex experience that individuals, communities and health providers find difficult to understand. Historical models of pain provide a legacy of understandings and misunderstandings conveyed in lay conceptualisations of chronic pain, and to a certain extent those of clinicians. Modern models of pain integrate biological, psychological and sociocultural factors, and, as a result, interventions have been developed to address these identified factors. Successful pain reduction is, however, difficult to achieve for many people with chronic pain, and it remains a substantial problem in most countries (Turk et al., 2011). This has led to numerous psychological approaches for helping people cope effectively with chronic pain. Coping approaches typically involve reconceptualising pain as something that can be managed, targeting beliefs and behaviour to reduce distress and disability, and promoting setting and achieving personal goals to gradually increase activity levels and enhance optimism. Programmes supporting individuals to develop selfmanagement skills are delivered in numerous settings, including individual, group, primary, secondary and tertiary health settings, and by a range of health professionals from disciplines including nursing, occupational therapy, physiotherapy, psychology and social work.

Two key limitations of current coping models for chronic pain exist. The first is that the specific elements of pain management programmes required for successful outcomes are unclear, and it is hard to know what works for whom. Some strategies have limited evidence for their effectiveness although they are often recommended, such as activity pacing (Andrews et al., 2012; Birkholtz, Aylwin, \& Harman, 2004 ; Karsdorp \& Vlaeyen, 
2009; McCracken \& Samuel, 2007b; Nielson, Jensen, Karsdorp, \& Vlaeyen, 2013), while some people may not continue using strategies developed during a pain management programme, yet continue to have lower levels of disability than prior to the programme (Curran et al., 2009).

The second is that very limited research has explored the ways coping approaches are used in daily life by people who look for help with their pain only rarely. Participants in the majority of pain research are from tertiary treatment settings and have been actively seeking treatment. The process of treatment seeking is not related only to pain intensity, but is also influenced by distress, degree of interference with daily life, appraisals of the meaning of pain, and by socio-cultural factors such as socio-economic status, and availability or past use of treatment. Those individuals who manage their pain effectively and thus present less often for treatment represent a group with different characteristics from those who seek help. They are more difficult to locate for research, which may explain in part why there are so few studies. Additionally healthcare has primarily focused on dysfunction, illness and disability, therefore, questions about how people cope well or how to support and develop positive attributes may not have received much attention.

A further complication, when considering ways to enhance coping with pain, are social attitudes towards pain. There is no doubt that these attitudes are fluid and that they influence expectations and behaviour in both patient and health provider as I demonstrated with an example of changing attitudes towards pain relief in childbirth. There are many people with chronic pain who do not respond to surgical, procedural or pharmacological approaches to reduce pain. These people continue to experience pain, and may continue to experience disability and distress because they have not been able to develop effective coping strategies that help them to remain engaged in valued roles and occupations. The literature abounds with descriptions of the factors associated with their difficulties, but is silent on those who live comparatively well with pain. 
Within this chapter I have outlined a modern understanding of pain as a multidimensional, biopsychosocial phenomenon, presenting several models of pain. I pointed out that attitudes towards pain are developed within a social context, and are very fluid. I have discussed some of the ethical issues associated with pain management including the social construction of clinician's attitudes towards pain and suffering. The various influences on treatment seeking were shown to develop in response to a combination of both personal and communal beliefs and behaviours.

When considering the ways people moderate their experience of pain, I have discussed several models of coping but pointed out that most of these models do not account for contextual variables and personal goals or motivation for coping. Coping models do not currently incorporate changes in coping approach over time (from acute pain to chronic disability). and classifications of coping approaches are not consistent, with some strategies classified differently in each taxonomy.

Finally, I identified the very limited attention paid to examining the ways people who limit their search for treatment live with pain. This gap in the literature is unsurprising given the focus of most attention is on people who look for treatment, but at the same time it means the resources, approaches and process used by individuals who are resilient are unknown. A deeper understanding of how people cope well with their pain in the context of their daily lives provides an opportunity to develop or refine the ways used to reduce disability and distress in those people who seek treatment. Understanding how people develop and maintain resilience could lead to more effective public health programmes for the problem of chronic pain. This may support shifts in attitudes/beliefs within the community, including those who seek treatment, but strategically more important, in those who provide treatment. Treatment providers who are more aware that people can live well (undistressed and not disabled) despite having chronic pain may have more confidence to 
guide the people they see away from fruitless treatment-seeking, and begin to actively engage in living well despite their pain.

Learning more about coping may also influence what is offered in chronic pain management programmes, improving the likelihood that skills will transfer into daily life when participants return to their own environments. This should help to reduce the direct and indirect costs associated with chronic pain, improve quality of life for those with chronic pain by reducing distress and disability, and enhance self-efficacy.

\section{Summary}

Within this lengthy chapter, I have discussed and defined pain within a biopsychosocial framework. I have considered the shifting perspectives and attitudes towards pain and pain relief, and by doing so I have indicated how flexible beliefs about pain can be. I have pointed out the limitations of current coping models which do not account for processes involved with adapting or accepting chronic pain, and may not apply to people with chronic pain who consider themselves to be well. Finally, I have justified the need for an explanatory theory to understand and learn from this group of individuals. Having established why this research question is necessary, I turn to consider the methodological issues relevant to exploring the question of "how do people who live well with chronic pain manage their pain on a daily basis?" 


\section{Chapter Three - Why use grounded theory?}

The pattern, and it alone, brings into being and causes to pass away and confers purpose, that is to say, value and meaning, on all there is. To understand is to perceive patterns... To make intelligible is to reveal the basic pattern.

Isaiah Berlin. (1997). The proper study of mankind: an anthology of essays, Chatto \& Windus, p. 129.

This chapter examines the purpose of developing theory in general and then, in light of conclusions drawn in Chapter Two, discusses the strategy I employed to answer my research questions. I then review the three leading forms of grounded theory and their philosophical assumptions. I follow this by justifying my choice of classical grounded theory for the empirical study of this thesis, before concluding with a review of quality indicators for appraising a grounded theory.

\section{Why develop a theory?}

Like so many concepts in science, there is a range of opinion about what constitutes a theory and how theories might be used (Sutton \& Staw, 1995, p.79). It is hard to argue with Lynham (2002) who states that the purpose of a good theory is to "explain the meaning, nature, and challenges of a phenomenon, often experienced but unexplained in the world in which we live so that we may use that knowledge and understanding to act in more informed and effective ways" (p. 222).

Theories can have multiple functions. A theory can be used to structure enquiry, apply and extend knowledge, and provide pragmatic explanations for phenomena (Wacker, 1998, p. 362). For example, a theory can enable academics and practitioners to identify where differences of opinion lie in the aetiology of a condition. In the fear-avoidance model, for instance, the relative contributions of health culture and significant others on maladaptive pain beliefs can be debated by clinicians and researchers because of common causal pathways similar to those implicated in depression suggested within the model (Pincus, 
Smeets, Simmonds, \& Sullivan, 2010). The fear-avoidance model and the analogous depression model provide structures for generating arguments for, or against, both views.

Theory also builds on, and integrates, existing knowledge, helping researchers to avoid following lines of inquiry that do not increase understanding or only generate solutions that have failed to work in the past. Vlaeyen and Linton (2012), in a recent review of the fear-avoidance model, highlight the progress that has occurred since their earlier paper in 2000 , in which they indicated a number of unresolved issues that required further exploration (Vlaeyen \& Linton, 2000, 2012). As they discuss the research that has emerged, they point out that early versions of the model drew upon fear conditioning research. This is supported in chronic regional musculoskeletal pain such as low back pain, but not as strongly in more generalised pain problems such as fibromyalgia. They recommend future research to explore alternative explanations, rather than to continue to investigate the fear-oriented paradigm. This call is reiterated by Wideman et al. (2013) who argues that some of the assumptions of the original model are not supported by the evidence, and in particular, the cyclical relationships between catastrophising leading to avoidance leading to disability and depression. This points the way toward the need for a new model, maybe even a paradigm shift (Kuhn, 2012). It is only by having a theoretical model clearly articulated and systematically investigated that researchers can make such recommendations.

Lynham (2002) believes theories can be used to explain and predict, and to increase understanding. He argues that the purpose of developing theory is to make implicit knowledge tacit, and so improve understanding and action (p. 223). For applied domains such as health, this seems a critical point. Theory should be useful. The aims of both qualitative research, with its focus on understanding, and theory development focusing on both prediction and understanding, appear compatible. 
Finally, theory can provide practical and logical arguments for clinical reasoning decisions when face-to-face with a patient (Haig, 2008a). For instance, when a patient describes avoiding certain movements in some contexts but not in others, based on the above model it is reasonable to consider pain-related fear, and to assess the context and presence of safety cues in different settings.

In view of my orientation as a clinician and researcher, an important reason for developing a theory to explain how people learn to live well is to further the knowledge of coping so that it can be applied in clinical settings. A theory of explaining the process of learning to live well with chronic pain should attempt to meet all of these requirements.

\section{What is a theory?}

Wacker (1998) considers "the definition of theory ... has these four components: definitions, domain, relationships, and predictive claims to answer the natural language questions of who, what, when, where, how, why, should, could and would" (Wacker, 1998, p. 364). To be useful for clinicians, a theory should (1) define relevant constructs within a domain of human structure or function (what, how, and why); (2) determine the relationships between the constructs (how, when, and why), and thus (3) determine why a clinically-relevant change has occurred, or the likely effects of influencing constructs or variables (who, where, should, could, would) (Ward \& Haig, 1997). As indicated in Chapters One and Two, while most chronic pain and disability research has examined how and why people develop problems when coping with pain, there is scant understanding of how and why people maintain function despite pain, and little explanatory theory is available. Theories explaining dysfunction may not explain why some people function well: the factors influencing positive coping may not be the inverse of those influencing disability. Some postmodernist writers dispute Wacker's definition of theory. Some writers question whether a theory should predict. Maggie MacLure (2010) considers that theory should 
"offend and interrupt" (p. 277), arguing that theory should stimulate new paths of enquiry, move beyond a stereotypical representation of the world (she uses the word cliché), to "stop us thinking that things speak for themselves" (p. 279). She says researchers simplify their experience, and in doing so adopt assumptions that can pass unnoticed. She prefers to deconstruct constructs to grapple more adequately with the complexity of what occurs in the real world. Deconstruction exposes assumptions and generates discussion, and the task of determining the implications of these debates is left to those who read theory or work with "real life" examples (MacLure, 2010, p. 283). Theory is therefore intended to highlight assumptions that might otherwise go unnoticed.

Theory has two main forms: accommodation where previous data (or phenomena) is incorporated and explained more adequately than in existing theoretical explanations; and prediction in which theoretical premises can predict phenomena that were excluded or unknown during initial theoretical development (Miller \& Fredericks, 1999). Some science philosophers suggest that prediction is more valuable than accommodation because a theory that has survived falsification has greater support than one that has not (Hitchcock \& Sober, 2004). Even without the falsification argument, understanding both when a law does and does not hold true can be valuable. For example, it is useful to have theory to understand how and why some people fear their pain and avoid activities they think will make it worse. It is also useful to explain why some individuals are not fearful of their pain and participate in activity that exposes them to pain, such as having surgery or engaging in physically demanding sports such as boxing (Bagraith, Strong, \& Sussex, 2012; Damsgard, Dewar, Roe, \& Hamran, 2011).

Hitchcock and Sober (2004) maintain that prediction is also more valuable than accommodation because it avoids the problem of 'overfitting' the data. Overfitting means drawing conclusions that cannot be generalised, or developing an overly complex theory because it takes into account very context-specific factors that are not found elsewhere - 
in other words, violating Occam's Razor "when you have two competing theories that make exactly the same predictions, the simpler one is the better" ("Occam's razor Wikipedia, the free encyclopedia," 2014). Douglas and Magnus (2013) discuss the importance of prediction in terms of epistemic assurance, agreeing with Hitchcock and Sober's point that prediction minimises the risk of overfitting data, but going on to suggest that prediction enables new phenomena to be discovered, or seen as relevant to a theory. They also argue that the productivity and reliability of explanations can guide us in the real world.

Within pain and disability theories, the fear- avoidance model has been tested and refined, generating a wealth of clinically useful information and demonstrating the value of a theory that can predict what happens in daily life. The fear-avoidance line of research also illustrates how useful it is to refine a model in light of new information. The theory has been extended over time, providing useful explanations for how and why some individuals develop profound disability when they experience pain. The model does not, however, explain why some individuals persist with activities despite pain, and neither does it explain the boom and bust activity pattern. These phenomena are now being explored within the framework of the fear-avoidance model, although efforts to integrate this concept and other domains have not yet been fulfilled (Damsgard et al., 2011; Hasenbring, Hallner, \& Rusu, 2009; Vlaeyen \& Linton, 2012). Some researchers are now calling for the model to be revised or revolutionised to use Kuhn's language, and this may in turn lead to a paradigm shift in conceptualising the ways certain individuals respond to pain (Bagraith et al., 2012; Damsgard et al., 2011; Kuhn, 2012; Pincus et al., 2010).

Theories or models structure information collection so that premises about phenomena can be developed. Researchers generate hypotheses to test the relationships between various factors thought to influence the phenomenon. In doing so, these relationships are clarified, additional variables are incorporated, and some variables become irrelevant. The 
relationships between variables also generate predictions about phenomena not yet observed. At the same time, theories must be flexible enough to accommodate new data as it emerges, to be applicable in enough settings for them to be useful, and also provide stimulus for debate and discussion.

\section{How are theories developed?}

Theory development is an indispensable aspect of science, yet the process of generating theory receives relatively little attention compared with theory verification (McGuire, 1997). Traditionally the process of developing a theory consists of either discovering a phenomenon (usually by identifying patterns in data), or by collating and synthesizing already-verified hypotheses into a coherent whole (Alvesson \& Karreman, 2007; Handfield \& Melnyk, 1998). The process of theory development has been called guesswork and theories that have not been tested, conjectures (Alvesson \& Karreman, 2007, p. 1265). When developing a theory, researchers often draw upon analogous explanations and incorporate logical deductive reasoning (working from the general to the more particular) or inductive reasoning (working from the specific to the more general) to generate hypotheses. Over time as researchers refine the theory, predictions become more accurate until the theory is sufficiently well-defined as to be a "law." This is well-known in subjects such as physics and mathematics, but seldom occurs in social science.

Thomas Kuhn (2012) argued that the scientific process is rarely as straightforward as this depiction. He prefers to describe it as a process of a paradigm shift. Kuhn describes periods of what he called normal science, during which theories are progressively modified to explain phenomena. Over time as an increasing number of anomalous findings cannot be incorporated into an existing theory, the status quo is challenged, generating the impetus for a new paradigm. Paradigms are new models or explanations that from emerge to revolutionise scientific understanding of a phenomenon, resulting in new concepts, language and ultimately, new theories (Kuhn \& Hacking, 2012). New theories emerging at 
this time generate more questions and pose new puzzles, eventually developing into another period of normal science.

In pain science, moving from biomedical explanations of pain to biopsychosocial ones exemplifies a paradigm shift. Aspects of pain that cannot be satisfactorily explained by biomedical reductionism (dualism) are better explained by dynamically integrating physiological, psychological and social factors (Gatchel, 2004; Jacob, 2003). There are many current theoretical explanations for the experience of pain and associated disability. These theories run the spectrum of purely biophysical through to sociological and have varying degrees of clinical utility. They are also modified continually in the light of new findings from both basic science and applied clinical research.

Theories from outside scientific research such as traditional Chinese medicine and complementary or integrative medicine theories also exist. I have not included or discussed these latter theories because they are rarely systematically evaluated using scientific principles and methodologies, and in some cases, appeal to principles that cannot be tested (Hartman \& Norton, 2002). Medicine or healthcare, is described as a scientifically proven evidence-base approach supported by robust data. In contrast, rather than being called "alternative", healthcare practices that have not been subjected to this level of scrutiny could be called "unproven" (Fontanarosa \& Lundberg, 1998, p. 1618). A model of pain that appeals to mechanisms that cannot be scientifically tested means adherents must have faith or believe in its tenets; such a model is less likely to produce interesting hypotheses for furthering knowledge than a model or explanation that can be comprehensively and systematically examined.

As I have shown, in spite of the wealth of research into the human pain experience, currently it is difficult to find explanations as to why some individuals cope well with pain, whether acute or chronic. Indeed, there are relatively few studies examining those who do 
not seek treatment, and those studies that are available, have not yet been integrated into a coherent model. It is this gap in the understanding of pain that I seek to address.

\section{The research purpose}

The purpose of this research is to develop an empirically-grounded theoretical explanation for how and why some people cope well with pain in daily life, to understand what they do, and to identify relevant factors influencing how and why they life well. An explanation should help clinicians develop practical ways to support this group of people, and to consider how this perspective on coping might apply to those who have more difficulty with their pain.

How this research question arose and how grounded theory may help answer it

As I have previously identified in Chapter Two, a group of around $15-20 \%$ of individuals with persistent pain do not routinely seek treatment, and neither are they distressed or disabled (Dominick et al., 2011; Karoly \& Ruehlman, 2006). These people are rarely referred to tertiary pain management centres, they, therefore, have not received much research attention and there is little to explain how and why they have developed the degree of resilience to what is usually represented as a major stressor.

\section{A personal perspective}

While working as a clinician, I have listened to many chronic pain patients describe their distress as they struggle to cope with chronic pain in their lives. Yet when I have met people socially, I often hear "Oh, but I have chronic pain, and I've kept on working". At this point, people may attribute their ability to stay active and well to their attitude towards pain, the exercises they do, the support from other people and perhaps the flexible attitude of their employer. 
Positive psychology research, independent living and strengths-based approaches to long-term health problems also appeal to me. I had difficulty finding research that integrated living well or positive approaches to chronic pain management. I began to think about how different chronic pain management might be if it was possible to build a theory based on the strengths of people I spoke to informally.

The factors that influence poor coping may be different from those factors involved in effective coping. While chronic pain research has focused on developing ways to improve how individuals cope, I wondered if the factors associated with living well with chronic pain were related to contextual factors such as social support or employment, or if other factors not yet incorporated into current theories of chronic pain might be relevant. It seemed important to begin studying how people progressed from finding out they had a chronic condition to the point when they began describing themselves as living well. Questions of how and why lend themselves well to grounded theory methodology, and I now discuss how the methodology can be used to build a theory to explain how people cope well with pain.

\section{How grounded theory might help answer these questions}

There are few systematic approaches to generating theory. While qualitative studies describe phenomena and fulfil the need to identify "relatively stable feature[s] of the world" (Haig, 2008c, p. 1020) commentators argue that many do not fully address how their findings can explain or contribute to theory (Holt \& Tamminen, 2010; Paley, 2011; Weed, 2009). Grounded theory methodology bridges the gap between qualitative description and theoretical explanations (Frank \& Polkinghorn, 2010; Shah \& Corley, 2006a; Skeat \& Perry, 2008) and appeals when a research question is about 'what' and 'how' people solve the problems in their sphere of action (Stanley \& Cheek, 2003). Glaser has argued that grounded theory is well-suited to complex fields such as health science, business and management (Glaser, 1995). Other commentators suggest grounded theory 
is useful when there are few or inadequate theoretical explanations for a phenomenon, or when the phenomenon involves a process or changes over time (Creswell, Hanson, Plano, \& Morales, 2007).

I sought a methodology that would provide a structured, systematic approach to generating a set of linked hypotheses to explain how and why a sizable minority of people with chronic pain think of themselves as living well despite their health problem. I did not have a strong theory to test and wanted to avoid testing weak theories. Weak theory can slow scientific progress because they explain little or need many assumptions and remodelling over time (Locke, 2007). I could not identify useful hypotheses to test because so few studies have provided clear, testable models for this population. Measures that may be useful in experimental studies are developed to explore factors associated with poor coping, and in populations of people who seek treatment. I also suspected that learning to cope with pain is a process rather than a set of stable traits because I could see similarities between the processes of adjusting to spinal injuries or other chronic disabilities and coping with chronic pain. Finally, in contrast to research conducted with people who seek treatment, locating people who cope well with pain is relatively difficult and it may be difficult to recruit sufficient participants to achieve statistical power to test a hypothesis.

Grounded theory is well-suited because it uses data collected from within a unique and relevant context. It has a cohesive logic of discovery and is recognised as being ideally suited to studying areas where little theory exists. It uses pragmatic criteria for establishing quality: does it fit, does it work, is it relevant and can it be modified? All of these are useful characteristics for a theory clinicians may want to use.

The next sections in this chapter outline grounded theory methodology, then provide an overview of ontological and epistemological issues that need to be considered when selecting the form of grounded theory to use. I follow this discussion by examining the 
differences between the three main types of grounded theory; discussing the relevance of symbolic interactionism and dealing with pre-existing knowledge. I then explore the ways in which grounded theory can contribute to theory development, and finally, comment on the ways quality can be judged in grounded theory research.

\section{Grounded theory and variants}

"Grounded theory is used to investigate problems of why and how in a systematic way, one that is 'grounded' in the data itself rather than being deduced logically or hypothetically" (Jones, 2009, p.30).

\section{Introduction to grounded theory}

Sociologists Barney Glaser and Anselm Strauss developed grounded theory when undertaking their 1965 study Awareness of Dying. Glaser first published a paper describing grounded theory in 1965, and later Glaser and Strauss detailed the process in their seminal work The Discovery of Grounded Theory and related writing (Glaser, 1965; Glaser \& Strauss, 1967; Glaser \& Strauss, 1965). Glaser has continued to articulate distinctions between what is now often called classical grounded theory and subsequent variations. Irrespective of the variant used, grounded theory is systematically generated from a broad array of data (i.e. grounded in data). While all grounded theory produces substantive theory that applies within a defined context, classical grounded theory can be further developed to form a formal theory that applies beyond the initial substantive area (Glaser, 1965, 1998, 2003; Glaser \& Holton, 2007a). Grounded theory involves abductively analysing data (often, but not exclusively, from interview), looking for "interchangeable indicators that emerge [to form] a pattern" (Glaser, 2011, p.30). Researchers select participants on the basis of characteristics required to satisfy the research question and develop the emerging theory, iteratively developing questions as abstract or conceptual categories emerge from the data (Charmaz, 2006a, p.29; Glaser, 2005). Analysis proceeds using constant comparison comparing data collected from different people, and data 
collected from people at different time points by comparing incident to incident, and interrogating the data by asking: "What category does this incident indicate?"; "What property of what category does this incident indicate?"; "What is the participant's main concern?" (Glaser, 1998, p. 140).

Researchers begin by using open coding to generate as many codes to capture what is going on in the data as possible. The researcher then delimits categories to only those that are relevant to the emerging theory in a process called selective coding (Glaser, 2005). Selective coding involves identifying the core category, then ensuring all subsequent coding relates to categories associated with the core category (Glaser, 1998; Glaser \& Strauss, 1967; Glaser, 1992). Theoretical sampling is used to search for data needed to develop categories entirely and establish relationships between categories. Sampling at this point is concerned with identifying participants or sources of data with the necessary characteristics required to develop the properties and dimensions of the category fully. Theoretical sampling enables each category to be fully saturated, with the full range of dimensions or characteristics identified until no new features are found(Glaser, 1998). Glaser describes theoretical sampling as the "where next" for data collection, "for what" codes, and "why" in memos (Glaser, 1998, P. 157)p. 157. The relationships between categories are also determined through constant comparison, although at this point, the researcher uses theoretical coding, drawing from a range of extant theories and coding families to explain completely what is happening in the data (Glaser, 1978, 2005). Classical grounded theory usually identifies a single core conceptual category from the data with any number of subordinate categories relevant to the core; other variants of grounded theory accept greater numbers of core categories.

Along with coding, the grounded theorist regularly writes memos. Memos are used to record thoughts, reasoning processes and for developing conceptual abstraction as the 
theory emerges. The researcher sorts memos once analysis is complete, using them when writing-up the analysis (Charmaz, 2006a, p.92; Glaser, 1998, p.193).

The end product of classical grounded theory is a multivariate conceptual theory consisting of a core category with associated subcategories (Glaser, 2011). The purpose of the resultant theory is to develop an explanation that accounts for relevant behaviour occurring within the substantive area (Glaser \& Strauss, 1967, p.30). Glaser strongly urges researchers to develop abstract conceptualisations rather than descriptions of what emerges (Glaser, 1998, 2011; Glaser, 2008). Concepts are abstract, more distant from the data than the representative incidents used to identify the common, unifying features. Description, in contrast and as described by Glaser (2008), "emphasises the getting the "voice" of the participants" (p. 4). Description provides detail, richness and presents unique data. Conceptualisation, on the other hand, captures the essential commonality underpinning a range of examples. (Glaser, 2008; Glaser, 1999).

Classical grounded theory is characterised by flexible coding practices, ensuring that categories progressively become more conceptual, and by treating what participants say as data alone without requiring further validation or checking for trustworthiness. Classical grounded theory is intended to be used irrespective of any scientific philosophical position. Glaser is clear that the classical form of grounded theory is atheoretical and can be applied to any kind of data (Glaser, 2005, p.127). Researchers using this methodology are freed from adhering to any particular philosophical school, although this freedom presents a risk to researchers not fully aware of the need to account for their philosophical assumptions.

\section{Ontology and epistemology}

Before discussing the two other variants of grounded theory, it is useful to consider the underlying assumptions about what is real within social research (ontology) and the ways 
we can know about it (epistemology). These assumptions underpin methodologies and methods used to collect data, and help to clarify some of the differences between the forms of grounded theory. Nathaniel (2011) says attending to the "first principles, assumptions and beliefs of a given philosophy" provide structure, logic and cohesion to a methodology (p. 187). Methods, or the practical steps for collecting and analysing data may be used within many methodologies, but how researchers use these methods influences the end product of research. Philosophical assumptions permeate how methods answer research questions, and subsequently determine how research findings can be used. If a researcher is unaware of the philosophical orientation within a research paradigm, they can stray from the internal coherence of a methodology and results can be illogical or even invalid. This is particularly salient in grounded theory where it is possible to conduct research using similar methods while the methodology as a whole follows a particular philosophy of science. Unfortunately, terms used in philosophy of science are often "fluid.... they are sometimes used interchangeably and positions can therefore be difficult to pin down" (Bryman \& Bell, 2011, p.16). I have simplified the following discussion in view of this and define the terms below despite ongoing philosophical arguments about these definitions.

Ontology is concerned with the existence of, and relationship between different aspects of what is real. A continuum can be drawn with realism at one end (which assumes there is an external reality outside of an individuals' perception of it), to constructivism at the other (which assumes there are multiple realities, constructed by individuals) (Ballinger, 2004; Weed, 2009). Realists seek to explore aspects of reality that may exist even outside the awareness of individuals. Data is considered to represent evidence for a phenomenon independently of individual perspectives. Constructivism "assumes that reality is neither objective nor singular, but that multiple realities are constructed by individuals" (Weed, 2009, p.507). Research with a constructivist ontology is concerned with constructing an 
understanding within a certain context such as gender, ethnicity, time, or cultural assumptions (Charmaz, 2006a, p.131).

While ontology is about what is and is not real, epistemology is about how to determine what is real. Epistemological assumptions can also be viewed on a continuum. At one end, positivist philosophy assumes that it is only possible to know the world through direct objective observation or measurement of a phenomenon. At the other end, lies interpretivism which believes that knowledge about the social world can only be understood indirectly because the individual alone knows his or her mind. Knowledge about social phenomena within this philosophy is inevitably an interpretation of what is happening. Somewhere in the middle of the continuum lies realism. Like positivism, a realist perspective acknowledges that social structures have some form of independent existence which individuals experience as external to themselves, but unlike the positivist view, realism acknowledges hidden mechanisms that can only be inferred from what is visible or observable.

Researchers using classical grounded theory consider that phenomena are discovered within the data and that these latent structures may not be evident to the individual research participant but can be identified by aggregating multiple indicators. The researcher seeks to understand what is going on from the point of view of the participant, but then abstracts from each instance to a latent variable or concept that may not be evident to the participants (Glaser, 2005, p.2; 2011, p.8). This position suggests that realist ontology can, but does not need to, be adopted when following this methodology because phenomena can exist separate from the participant's perspective. It is essential to note that Glaser and Strauss never formally labelled their methodology with any ontology or epistemology while Glaser repeatedly states that classical grounded theory "can be used on any data type and with any theoretical perspective" (Glaser, 1998, p.41). 
Glaser and Strauss developed the original methodology at a time when philosophy of science was not being debated extensively, and also at a time when sociologists carried out fieldwork analysis with a pre-determined existing theoretical framework. The late 1960's were a time when "positivist" research methodologies were increasingly popular in social scientific studies. Qualitative research was considered exploratory, "impressionistic" and unsystematic (Ong, 2011, p. 418) while hypothesis testing was given more attention than generating theory. Glaser and Strauss believed that researchers were conducting valid research when they were in the field collecting data, and proceeded to describe the implicit coding" they believed was being carried out when a researcher was coding (Glaser \& Strauss, 1965). By being more systematic about the ways in which data collection, coding and comparison groups were carried out, Glaser and Strauss hoped to convey the rigour and usefulness of theory generation. The aim was to ensure that researchers produced theories that were relevant to the fields in which they were generated, rather than being abstract and irrelevant (p. 5).

Despite Glaser's (2005) assertion, various writers have identified his version of the methodology as realist or perhaps critical realist in orientation (Nathaniel, 2011; Oliver, 2012; Weed, 2009). Nathaniel (2011) further suggests that classical grounded theory is consistent with Charles Sanders Pierce's pragmatist philosophy (p. 188). In pragmatism, the practical consequences of an idea or proposition determine its truth. Nathaniel (2011) suggests that although Glaser and Strauss were not particular about their position and argues close reading of Glaser's writings show similarities with Pierce's pragmatist perspective. Labels applied to grounded theory include "realist, constructivist, critical realist, objectivist, relativist, interactionist, positivist, post-positivist" (p.192), but Nathaniel argues that Glaser acknowledges an objective and external reality that is independent of the participant. Glaser himself challenges researchers to be a "non-citizen" while carrying 
out research to allow the data patterns to become evident rather than being clouded by the researcher's own beliefs or orientation (Glaser, 1978, p.8).

More recently, Glaser writes that what participants say should not be considered "interpretations", but are simply data from their perspective (Glaser, 2011, p.9). He points out that researchers hold a different perspective from participants because researchers listen for patterns among participants and situations. Grounded theorists seek patterns that repeatedly occur in the data, whether this is an interview, text or quantitative data (Glaser, 2005, p.127). Researchers are, he argues, finding emergent patterns and naming them (Glaser, 2011, p.9).

Glaser's view of theory development is similar to Pierce. Glaser and Pierce are both concerned with conceptualisation (Glaser, 2008; Nathaniel, 2011). Pierce considered that concepts were a structure within which to organise individual observations or pieces of information. He believed that concepts help to reduce the volume of information by grouping items with similar properties together (Peirce, 1867). Constant comparison is used to identify repeated patterns or similarities within the data, thus reducing the information into a category or concept with a range of indicators that illustrate similar properties (Glaser, 2011, p.9). The researcher simplifies and coheres a variety of instances that represent an underlying commonality.

The philosophical stance a researcher adopts will influence his or her choice of methodology when answering a research question. Methodology determines the techniques or methods used to collect data. The inferences, that can be drawn from the findings, depend on methodological assumptions. Philosophical assumptions and how these influence both data collection and use are apparent when the two other main variants of grounded theory are examined, and I will discuss them both in the next section. 


\section{Grounded theory variants}

The original collaboration between Glaser and Strauss began the development of grounded theory, but the pair eventually differed in their accounts of the methodology.

Straussian grounded theory involves using a highly structured set of procedures for coding, memoing, and diagramming as the researcher works to study the boundaries between data and categories. Strauss and Corbin developed a series of questions about the conditions, actions/interactions, and consequences of categories, consequently making links between the ideas or concepts identified from the data (Strauss \& Corbin, 1994). Novice researchers have adopted Straussian grounded theory perhaps because of the structure inherent in these procedures, in contrast to the less structured approach of Glaser (Charmaz, 2006a; Miller \& Fredericks, 1999; Weed, 2009). Straussian grounded theory has several elements that differ from Glaser's methodology. Glaser uses open, substantive and theoretical coding while Strauss includes open, axial and selective coding. In Strauss and Corbin's method, open coding involves micro-analysis word-by-word; axial coding relates categories and properties to each other, using a combination of inductive and deductive thinking; and selective coding is the process by which all categories connect to the core category. Straussian grounded theory originally had a strong emphasis on verifying categories and relationships in the data by first generating meanings from words or phrases, then verifying their presence within subsequently collected data (Corbin \& Strauss, 1990a; Strauss \& Corbin, 1994). More recently, Corbin has written about her changing perspective, and acknowledges that all of the techniques presented in their textbooks may be omitted, or used less rigorously than initially presented (Corbin $\&$ Strauss, 2008b).

Glaser uses inductive reasoning (often identified as abductive reasoning) while the Straussian approach seeks agreement between the researcher and participants. The particular context and assumptions, that both participants and researchers bring to 
research, can constrain the findings using this approach, and it may be difficult to apply them to other contexts. It also poses the question of what to do if the various participants do not agree either with the researcher and the emerging theory or with each other (Walker \& Myrick, 2006b). Glaser and other authors challenge the use of verification, arguing that this moves away from relying on the constant comparative approach, and instead becomes a prospective search for data to confirm or verify the categories identified. Glaser and Strauss' original publication states clearly that grounded theory does not require further verification because the propositions are derived directly from data (Charmaz, 2006a; Glaser \& Strauss, 1967; Glaser \& Holton, 2004; Heath \& Cowley, 2004). Glaser further argues that it is fruitless to check emergent theoretical categories with participants because they may not be aware of the latent variable identified by the researcher (Glaser, 2011, p.9).

One commentator considers Strauss and his collaborators are "epistemologically interpretivist ... ontologically realist..." (Weed, 2009, p.508). This position can be called "post-positivist" and assumes that while some parts of the social world cannot be directly measured and is therefore interpretivist, features of the social world can be identified independently of the individual, and is therefore realist. Strauss and Corbin, however, explicitly stated that they adhere to both an interpretivist epistemology and ontology (Strauss \& Corbin, 1994, p. 276), again their position shifted in later writing (Corbin \& Strauss, 2008b).

A final major variant of grounded theory to be discussed in this thesis is by Charmaz (Charmaz, 2006a). She views the methods and procedures used in grounded theory as ontologically and epistemologically neutral, and able to be used by researchers from either realist or constructivist leanings, but then states that researchers "... construct our grounded theories through our past and present involvements and interactions with people, perspectives, and research practices" (Charmaz, 2006a, p.10). Her approach thus 
differs from both Classical and Straussian grounded theory in that it is unashamedly constructivist and interpretivist. Charmaz rejects the procedures detailed by Strauss and Corbin, but incorporates several strategies common to constructivist research such as returning to participants to check the accuracy or adequacy of the emergent theory, and being explicit about the researcher's values, beliefs and presuppositions (Charmaz, 2006a, p. 131).

Grounded theory continues develop, and variations or clarifications (depending on particular viewpoints) are still emerging. It is beyond the scope of this thesis to examine every iteration of what has been identified as "a type of central organising heuristic for data analysis and interpretation" (Miller \& Fredericks, 1999), but while there are many studies that indicate grounded theory methods have been used, the methodology is intended to be a complete methodology rather than merely a method. Methods are the techniques or tools used to conduct research, such as sampling strategies, data management or data analysis processes; while methodology refers to the theoretical underpinning of methods used to explore a research question in a coherent manner and to justify the methods chosen. In grounded theory, using constant comparison to identify themes in qualitative data without subsequently developing categories and properties of categories in relation to one another equates to using a method, rather than using grounded theory methodology.

\section{Grounded Theory and Symbolic Interactionism}

Glaser contends that classical grounded theory does not require researchers to adopt a symbolic interactionist perspective (Glaser, 2005, p. 141). Grounded theory has often been construed as positioned within this view. Symbolic interactionism was founded by George Herbert Mead (1863-1931). While Mead's concepts were more philosophical than applied, Blumer (1980) moved symbolic interactionism towards an applied sociological theory and research approach and informed by American Pragmatism (Jeon, 2004). It is a theory that 
focuses on the ways humans interpret or define one another's behaviour in terms of meanings or symbols that are subject to interpretation. The central tenet of symbolic interactionism is that it is through interacting with the world that humans make sense of it, and subsequently construct meaning. In symbolic interactionism, the social meanings of objects in the world are "the most important predictors for human behaviours" (Jeon, 2004, p. 250), and humans learn not only about objects but also one another by social interactions, and in so doing develop social rules about what other people are doing or what they are willing to do. Humans consequently alter their own actions by taking into account what others do, in an iterative fashion (Jeon, 2004). It has been seen by many commentators as an essential perspective in grounded theory, and it is certainly explicit in Strauss and Corbin's early accounts of grounded theory (Corbin \& Strauss, 1990b), as well as in Charmaz' constructivist grounded theory (2006a).

Glaser has indicated that Strauss draw on symbolic interactionism as a result of his training in Chicago (Glaser, 2005, p.142). Glaser's view, however, is that classical grounded theory is "...a relatively simple inductive model" that identifies concepts from indicator events within the data by using constant comparisons (Glaser, 2005). Glaser rejects using symbolic interactionism as a founding principle, and instead states that the methodology can be used "on any data type and with any theoretical perspective." (Glaser, 2005, p.144). Despite Glaser's position, some of the assumptions of symbolic interactionism are present in this thesis. Examples include the interaction between researcher and participant in which as a researcher, I have developed an understanding of the way certain words are used by participants; the experience participants have of their pain and their lives as a fluid reality, where change is the only constant; the way participants altered their view of their pain as they experienced interactions between themselves and their healthcare providers. At the same time, as I will explain in the next section, concepts drawn from symbolic 
interactionism have had to 'earn' their way into the theory, as have other perspectives from the extant literature.

\section{Dealing with pre-existing knowledge, and extant literature}

A further difference between Glaserian and other schools of grounded theory is how preexisting knowledge and the literature is handled by the researcher. In all three forms of grounded theory, the researcher must remain open to what is present in the data, and to avoid "forcing their data into preconceived codes and categories" (Charmaz, 2006a, p.67; Glaser, 1978, p. 31; 1998, p. 69; 2005, p. 5; 2011, p. 27).

Classical grounded theorists believe all is data, and add findings from the literature, as well as previous knowledge held by the researcher to the grounded theory as it develops, but after having identified concepts directly from the "actors in the field" (Glaser, 1998). Previous knowledge sensitises the researcher to what might be, but the way the researcher approaches information is with curiosity rather than holding preconceptions of what it 'should' show. Glaser argues that a researcher's openness to what might be present can be compromised by conducting a detailed review of the literature prior to collecting data (Glaser, 1998). Charmaz, on the other hand, suggests that prior knowledge in the form of sensitising concepts and disciplinary perspectives "provide a place to start, not to end" (Charmaz, 2006a, p.17).

The key method to achieving this 'spirit of inquiry' in Glaser's approach is by using memo notes, or ongoing records of thoughts, musings and ponderings as the researcher collects and analyses material from various sources. Glaser indicates that the researcher may conduct an 'interview' of him or herself as part of the data collection process, using data from this, in the same way as any other data (Glaser, 1998).

When using classical grounded theory, a sensitising concept provides a springboard for conceptual leaps towards data that may not be thought immediately relevant. A study may 
begin with an interesting idea that is perhaps grounded in the researcher's experiential knowledge or literature, but the area under investigation rapidly takes on a life of its own as analysis progresses and becomes more conceptual (Glaser, 1978, p.39).

Strauss and Corbin, along with other researchers following the Straussian approach have quite a different approach to pre-existing knowledge (Corbin \& Strauss, 2008b; Walker \& Myrick, 2006b). Strauss and Corbin (1990) suggest that specific knowledge and understanding may be used to stimulate sensitivity and generate hypotheses. According to Heath and Cowley (2004), Strauss and Corbin go as far as to suggest that the research question should identify the phenomenon to be studied, and what is already known about it (Heath \& Cowley, 2004; Strauss, 1998)

The risk with this level of specificity is that the researcher's interests and preconceptions may influence the questions and even what the researcher notices (Glaser calls this forcing the data) rather than identifying new and unexplored explanations (Glaser, 1998; Richardson \& Kramer, 2006). Cognitive biases in human reasoning support his contention (Gilibert \& Banovic, 2009), although it is necessary to point out that opportunity for bias also occurs when using Glaser's less structured approach. Bias occurs because no person enters a research field without a research question in mind; researchers bring knowledge from other fields of inquiry and general knowledge and experience, and finally because the researcher's gender, ethnicity, socio-economic status, political persuasion and family/whanau heritage contexts can also exert influence. This is true, of course, for any social science or indeed research endeavour, although often hidden by shared language, definitions and procedures (Charmaz, 2006a; McGrath \& Johnson, 2003).

Charmaz appears to support Glaser's approach to incorporating extant literature (2006a). She considers Henwood and Pidgeon (2006) term theoretical agnosticism is a useful stance to take throughout the research process and suggests researchers "consider 
treating extant concepts as problematic and then look for the extent to which their characteristics are lived and understood, not as given in textbooks" (Charmaz, 2006a, p.166).

The ways in which grounded theorists consider existing theory and literature have been compared to the ways clinicians approach assessing patients. Clinicians are sensitised to potential phenomenon through their training, the literature, and their own theoretical preferences (Vertue \& Haig, 2008). Clinicians are trained to collect information from a wide range of domains before determining that they have detected a clinical phenomenon, or developing an explanatory theory for that phenomenon (Vertue \& Haig, 2008). Similarly, it is possible to be sensitised by existing knowledge but not tempted into premature theoretical explanations provided that the researcher retains an inquisitive and open view and can avoid forcing data into particular patterns, and provided constant comparison and memo-writing are used faithfully. I have followed these procedures in this research study, for while it is impossible to eliminate the influence of what I have read and considered in clinical situations altogether, it is possible to listen and develop an understanding of what is really being said during an interview, to paraphrase Glaser, leaving analysis until later (Glaser, 2008). This allows time to give due consideration to data drawn directly from participants, and information obtained from the research literature. In addition, Glaser argues that concepts must pattern out, or repeatedly occur in the data; thus there are protections against forcing the data to fit preconceived ideas (Glaser, 1998).

\section{Grounded theory and theory building}

Irrespective of the type of grounded theory, the methodology generates new theoretical explanations for a particular aspect or aspects of human behaviour. In doing so, it emphasises the first half of theory building, or the process of generating new hypotheses (Haig, 2008b; Mintzberg, 2005, p. 3). 
Theory development and testing are integral to much scientific endeavour, particularly positivist or realist research. Lynham (2002), although discussing Human Resources Development, considers that all applied disciplines need theory

to explain the meaning, nature, and challenges of a phenomenon, often experienced but unexplained in the world in which we live, so that we may use that knowledge and understanding to act in more informed and effective ways (Lynham, 2002, p. 222).

Developing theoretical explanations consists of the following broad steps:

1. A researcher makes an intriguing observation.

2. After repeated observations, the researcher confirms the observation is an "empirical regularity", or in other words, not simply a random or chance occurrence.

3. The researcher generates a range of possible explanations to explain how and why the observations occur.

4. Hypotheses are tested to determine whether the relationships between postulated variables hold true and under what circumstances.

5. The researcher integrates these propositions to form a coherent explanation for what was observed.

6. Theoretical explanations are then expanded and refined to establish how widely applicable the theory may apply, and to modify individual propositions where the empirical findings do not hold (Haig, 2008a; Lynham, 2002).

This process is not without its critics. As mentioned earlier in this chapter, Alvesson and Karreman (2007) consider that theory develops from "speculative thinking or empirical observations (followed by careful analysis)", calling Popper's description of theory creation as guesswork, or conjectures (p. 1265). They suggest that theory emerges from a time and place in history so that context shapes not only the theory, but also the way in which researchers view data or empirical material. Alvesson and Karreman suggest using 
empirical material in problematising theoretical ideas, or challenging the value of a theory and to examine its weaknesses and problems relevant to the phenomenon in question (2007). They argue that theory development can be facilitated by considering the aspects of what does not work in a theoretical explanation.

In pain research, theoretical explanations for disablement from persistent pain are modelled and remodelled over time. The fear-avoidance model proposed by Vlaeyen and colleagues (Vlaeyen, Kole-Snijders, Rotteveel, et al., 1995) was developed from work by Philips (1987), itself based on drawing an analogy with theories based on behaviourism to explain avoidance behaviour in individuals with phobia. In the years since the model was first presented various aspects of the model have been studied, resulting in several modifications to the original structure; adding additional constructs, and altering the definitions of others; until most recently, the model was extended by a group of researchers including the original authors to incorporate a motivational perspective (Crombez et al., 2012). Although the original inspiration for this well-known model arose from observing the pain behaviour of individuals with low back pain and by drawing parallels with avoidance by people with phobia, over time researchers have problematised the model and remodelled it to better explain why some people avoid movements in one context but not in another. Eventually, researchers hope this will enable it to explain and predict progress in treatment, and to move away from relying on a psychopathological explanation for the phenomenon when empirical findings do not fit readily into this framework.

Grounded theory provides one way to generate explanations (Egan, 2002) although it produces substantive theory, or theory that is limited to a particular domain of inquiry (Weed, 2009, p.506). From substantive theory, two further options are available: (1) formal grounded theory may be developed, or (2) propositions drawn from the grounded theory may be formally verified, perhaps using hypothesis testing and quantitative data analytic 
procedures. Formal grounded theory is a more abstract level of theory than substantive theory and is developed from a group of substantive theory studies about the same phenomenon or core category. Categories are conceptual, therefore, a core category found in one setting will likely be found in others, with any differences between contexts used to generate further coding in relation to the core category (Glaser, 2011). A view of the world that considers there are underlying similarities between different contexts lies closer to a realist or positivist view than a constructivist or interpretivist one. Researchers who are adhering to constructivist ontology, on the other hand, consider that both formal grounded theory and hypothesis testing are incompatible with the notion that knowledge is constructed by the participants and researcher. Weed (2009) argues that to move from substantive theory to formal theory would need "some acknowledgement of an underlying reality across substantive areas" and goes on to say that the constructivist variant of grounded theory may "be limited to producing only substantive theories" (Weed, 2009, p.508).

Turnbull (2002) challenges this view, describing social constructivist researchers as "us[ing] theory to interpret the social world". She considers that instead of being concerned with validity, reliability and generalizability, the theory produced by qualitative researchers should organise experience or observations to shed light on a situation, or suggest an area for action (p.319). Social constructivist researchers are thus more interested in the sense-making by people involved in the area of study and using the insights gained from situation-specific research to reflect upon existing theoretical frameworks or other, similar, situations. The work of determining what is applicable from one constructivist study to another context is left to the person who consumes or reads the research, rather than by appealing to an external, neutral, reality.

Although not usually stated as explicitly as in constructivist research, positivist or realist research also relies on research consumers to draw conclusions about the applicability of 
the findings from one context to another. For example, in pain research, studies examining the outcomes of chronic pain management programmes require researchers to detail the characteristics of the participants carefully, ensuring measures and procedures are followed so readers and clinicians can determine how closely the findings can be applied to another group of people with chronic pain. The work of interpreting whether the findings from a study using a positivist/realist perspective apply to a different population still must be carried out by those reading (and therefore interpreting) the published article. Miller and Fredericks (1999) ask, "Once you have produced a grounded theory, what variety of theory do you exactly have." (p.539). They go on to describe grounded theory as neither accommodative nor predictive. Accommodation involves explaining or interpreting data by demonstrating that it is consistent with extant theory. Prediction, on the other hand, is described by Miller and Fredericks as "... plausible but unknown states of affairs, which if confirmed as genuine predictions, enhance the credibility of the theory" (p.544). Their final conclusion, perhaps unhelpfully, is that grounded theory is "a type of theorizing ... distinguished by focusing on both the context of discovery and an inductive argument for its plausibility" (Miller \& Fredericks, 1999, p. 550).

Grounded theory explains on the basis of inference to the best explanation, which is, in simplistic form, accepting a hypothesis on the basis that it provides a better explanation of the evidence than is offered by alternative hypotheses (Miller \& Fredericks, 1999, p. 550; Thagard, 1978, p. 77). Thagard identified criteria for evaluating whether a hypothesis provides a better explanation, incorporating consilience (the breadth or range of facts explained, and how well the theory can explain new facts as they emerge), simplicity (the fewer special ad hoc hypotheses required), and analogy (similarity between explanations in a theory and familiar explanations, or concepts used in other established explanations) (Thagard, 1978, p. 79). 
When writing up a grounded theory, researchers draw on extant theory when exploring similarities and differences between what others have discovered elsewhere and the substantive area of the grounded theory. Researchers using classical grounded theory are invoking inference to the best explanation, and the resultant theory can be evaluated using Thagard's criteria to determine whether the theory provides 'a better explanation'.

\section{Meeting quality criteria for grounded theory studies}

Grounded theory has been criticised by many writers because of a perceived lack of clarity about epistemological and ontological assumptions by the originators of the methodology, and concerns related to modifications made in revisions of the methodology to make the process more easily followed or to fit the methodology to a prevailing philosophical position. Grounded theory originated at a time in sociology when researchers began fieldwork with a theoretical framework in mind, writing copious field notes but analysing these only after returning to write their findings. Discourse about philosophy of science had not yet touched upon the world of social science (Heath \& Cowley, 2004; Kearney, 2009; Kelle, 2007b). Glaser and Strauss may have omitted referring to any specific philosophy of science because of this context, although Glaser is very clear when he states "GT is not defined by and not the hand maiden of any type of data, any particular theoretical codes, any particular discipline, and/or any one theoretical perspective" (Glaser, 2005, p. 127). The grounds for determining validity, reliability, trustworthiness or rigour of the findings and resultant theory develop from adhering strictly to Glaser's processes although these terms are not typically used. Instead, grounded theory uses concepts of fit, work and relevance as criteria for determining quality.

There is a strongly pragmatic flavour to these terms. Glaser discusses validity and associated terms under the title of "trust", as in whether grounded theory findings can be trusted, and also "product proof". He argues that grounded theory's rigorous relationship 
between data and theory means findings derived from theory can be trusted (Glaser, 1998, p. 236), and the proof is in the outcomes of fit, relevance, workability and modifiability.

Beginning with fit, a concept that he equates with validity, Glaser uses pragmatic criteria to establish what other methodologies call "trustworthiness". Fit refers to how well the concepts represent data patterns obtained from the substantive area (Glaser, 1998, p. 236)

p. 236. Grounded theorists establish fit by generating concepts directly from data, modifying the name used to denote the emerging pattern by using constant comparison. Names for concepts within this Ph.D. study have changed in the process of constant comparison, clarifying the concept as various aspects of the category is developed. For example, the concept "flexibly persisting" began as the term "getting on with it". Getting on with it was often used by participants and had immediate grab. Over time, and in response to discussions with colleagues, feedback from the thesis examiners, and by gathering more data, dimensions of the concept were established and I became aware that the concept was more complicated than this. "Getting on with it" did not encompass how people carried out their lives in the way I had identified. The features of changing focus, adapting to different situations, yet retaining consistent direction and determination were not captured. Flexibly persisting caught these aspects more clearly than using the words participants had used.

Relevance is a second criteria and refers to "what is really going on" in the data (Glaser, 1998, p. 236). When data is obtained directly from participants engaged in the substantive area, emergent concepts will relate to those issues participants believe are most important. It is hard to deter people from talking about their main concern and how it is resolved, especially when questions are open-ended and designed to "instil the spill" (Glaser, 1998, p. 111). Emergent concepts are important to people in the substantive area, and confirmation of importance emerges with each interview and when discussing 
findings with others who fit the selection criteria for participating in the research and clinicians who work in the area.

When concepts fit and are relevant, the next criterion is whether the theory works. Does the theory account for "most of the variation of behaviour in the substantive area" (p. 237). The core category and sub-categories integrate to explain how the main concern is resolved and can, therefore, be used within the area. Relevance means that the theory should explain something important, something that has grab. Finally, modifiability means that the theory can be amended to accommodate new data as it emerges, with the theory continually being modified to integrate new or contradictory data (Glaser, 1998, 2011; Lomborg \& Kirkevold, 2003).

Glaser adds three additional sources of trustworthiness in grounded theory. In addition to fit, work, relevance and modifiability, he argues that grounded theories have temporal trust (Glaser, 1998, p. 235)p. 238. What he means by this is the immediate applicability of a grounded theory. The term used to label a concept have direct appeal and those reading the theory can relate to the concept quickly. Grounded theories have grab so, for example, the concept flexibly persisting conveys the notion of persevering with a goal, or in a direction, but using any number of ways to do so. Glaser goes on to say that grounded theory is free of time and place because it is conceptual (Glaser, 1998, 2011). The idea of flexibly persisting is likely present in human behaviour in many different settings and has probably been present since humans began needing to achieve self-coherence.

Consequently, grounded theories are durable over time. The same patterns of human behaviour are repeated.

Grounded theories are valuable because they can be used, modified as new data comes to light, and explain real world situations. Glaser indicates grounded theories have collegial trust (Glaser, 1998, p. 240)(p. 240). He believes colleagues can rely on grounded theory findings and do not need to repeat or verify the findings but can instead immediately apply 
and revise as new data arises. Glaser indicates that grounded theories are readily generalizable to other substantive areas and by using emergent fit (ensuring the original theory is constantly compared with data collected in the new substantive area) develop new properties of categories which must also show fit, workability and relevance. He believes the core category is likely to become a sub-category within another substantive area, but also points out that by using emergent fit the theoretical code within a grounded theory may become more apparent (p. 242). For example, a theory of living well with chronic pain explains re-occupying self, but a theory of those who have trouble living well may find a code of fragmentation, indicating that dealing with chronic pain can involve a continuum of coping.

Having discussed collegial trust, Glaser moves to layman trust. This notion is embedded within the usefulness or workability of a grounded theory. A grounded theory should not only explain a substantive area but should also help those within it. Important concepts are identified and can then be influenced by applying the grounded theory (Glaser, 1998, p. 247)(p. 247). This means grounded theory works well within applied science fields such as healthcare, design and computer engineering. Scientists or researchers may develop the theory, but laypeople can use the findings because they are relevant to the day-to-day problems they encounter.

Williams and Morrow (2009) address issues of validity and reliability in qualitative research. They examine the concept of trustworthiness, a concept that they believe can raise this type of research above journalism or anecdote (p. 576). They present three ways in which a reader can decide whether a piece of research is trustworthy.

The first is integrity of the data - that there has been sufficient data collected, and that others can understand, albeit not replicate, the processes used to analyse the data. They suggest that clear description of the analytic strategy be included in any documentation, quoting Patton (2002) who described this as "a systematic process systematically 
followed", so that while it is unlikely anyone could replicate the sample exactly, the processes used should be (Williams \& Morrow, 2009). They then concern themselves with ways to establish that sufficient information has been gathered and suggest sampling "diverse perspectives", "diversity of demographics", and use of "triangulation" including obtaining feedback from colleagues as strategies that may help ensure "richness and complexity" of constructs is present. Finally, they suggest that evidence as to how interpretations "fit" the data should be demonstrated, giving the use of direct quotations as examples.

At this point, it is worthwhile reflecting on the distinction between the aims of classical grounded theory to achieve conceptual depth and other qualitative methods more concerned with thick description. Classical grounded theory seeks to discover latent variables present in the data, and then to delineate the relationships between the core category and the other categories through theoretical coding. The data represent individual examples or indicators of latent variables, and thus are not intended to be viewed as separate from the concept they indicate. Data is closest to the context, while concepts are abstractions and theoretical relationships even more abstract. Quotations, are used sparingly, if at all, when defining a category because they bring the focus back to the particular rather than remaining conceptual. Glaser writes at length about the need to be conceptual when developing grounded theory (notably (Glaser, 2011). Conceptualising distinguishes classical grounded theory from other forms of qualitative data analysis, including the other variations on grounded theory. Concepts provide a way to simplify the world; details and examples derail this process.

Williams and Morrow also suggest sampling processes that are not consistent with the classical grounded theory methodology (Williams \& Morrow, 2009, 9.577)(p. 577). Purposive sampling in classical grounded theory is determined by the need to ensure as much variability within each category as possible, and sampling continues until no further 
fruitful variation can be identified (saturation). Thus, sampling is based on the emerging theory.

The next criterion for trustworthiness lies in ensuring balance between what the participants say, and the researchers interpretation. Because bias is inherent in any research from the moment a research question is asked in a certain way, within a certain context, by a certain researcher, for certain reasons (Williams \& Morrow, 2009, 9.579) (p. 579). Williams and Morrow suggest qualitative researchers make their subjectivity explicit through "bracketing" their biases (or knowledge base) prior to commencing their research, completing research journals, and as Guba and Lincoln (1994) suggest, "member checking" or confirming the research findings with participants through feedback.

During the research process, I completed an "interview with myself" (Glaser, 1998, p. 122) before beginning data collection, and I document my perspectives in Chapter Four. I have also incorporated memos completed at various points during the research project in Chapter Four. By doing so, I hope to have revealed some of my personal biases and any preconceptions.

Williams and Morrow (2009), and other qualitative researchers such as Lincoln and Guba (1994), (Reis, Hermoni, Van-Raalte, Dahan, \& Borkan, 2007; Shah \& Corley, 2006b) believe that in order to verify or otherwise ensure the researcher has accurately understood the participant's perspectives suggest checking the findings with participants. Some writers have expressed concerns about this process, arguing that it introduces another layer of analysis to the research, and recognising that it is not an infallible process (Elliott $\&$ Lazenbatt, 2005). Other authors, including Glaser (1998) and Strauss and Corbin (1994), consider that constant comparison and theoretical sampling throughout the research process, as well as the use of memo writing, provide a rigorous checking process to ensure that emerging concepts remain relevant as further data is collected (Elliott $\&$ Lazenbatt, 2005; Glaser, 1978, 2005, 2011; Glaser, 1992). 
It is clear that it is impossible to begin a piece of research with "an empty head", but my interpretation of Glaser's writing, and drawing on Kelle (2007b), Henwood and Pidgeon (2003); Oliver (2012); Thomas and James (2006), is that by remaining open to what is present in the data, coding, and writing memos throughout the process allows concepts that are relevant to the "core category" or "main concern" of the participants to remain in the model, while others are abandoned because their relationship to the core category is tenuous. If "all is data", as Glaser states, findings from existing research are included if, and only if, they have relevance to the core category. Extant theory and prior research serve to sensitise, or act as a frame or heuristic for considering tentative concepts, to be confirmed only as more data is collected. This is, in essence, the way grounded theory is "grounded" in the data collected throughout the study.

The final aspect considered by Williams and Morrow (2009) refers to the utility of the research and refers to how well the research is communicated, and then applied. Their argument is that by communicating the purpose, process and product of research social validity is achieved. Social validity is a term coined by Wolf (1978) and refers to how important a concept (or intervention) is, and whether it has achieved its goals in terms of value for the stakeholders. To achieve social validity, the research must be easily understood and supported by quotations from participants; demonstrate how the research question has been answered, and to ensure the context in which the research was conducted, is also conveyed (Williams \& Morrow, 2009). As in each of the other two methods for ensuring trustworthiness, grounded theory handles this concern slightly differently. By enquiring about participant's main concern, and generating a theoretical explanation for how this is resolved, Glaser contends that classical grounded theory is relevant and valid (Glaser, 1978, 1998, 2011), in particular, Chapter 8 of Glaser (1998). His argument lies in the need to examine the problems as identified by participants active in the area because the primary motivation for their behaviour is to resolve their main 
concern. A theory of how the main concern is resolved provides conceptual power and strategies for intervening (p. 116).

\section{Summary}

As I have shown, grounded theory of any type help generate new theory by exploring, and to some extent decontextualising, categories and relationships between categories. I selected classical grounded theory because it is compatible with a critical or scientific realist perspective which sits well with my philosophical position. My decision is based on understanding that there are concepts within pain and coping that appear in a number of different contexts such as the existence of a process of learning to cope with pain (Affleck, Urrows, Tennen, \& Higgins, 1992); people with chronic pain appear to have less disability when they have access to a broad range of coping strategies (Esteve, Ramirez-Maestre, \& Lopez-Martinez, 2007); and based on the value humans place on occupation (Reinseth, Kjeken, Uhlig, \& Espnes, 2012), suggesting that a realist perspective may be applicable. The argument that a researcher discovers then conceptualises and integrates ideas in ways that individual research participants may not, is also compelling and fits with my clinical experience. At the same time, classical grounded theory offers a flexible methodology for learning about "how" and "why" and allows the "discovery" aspect of theory development to proceed without focusing on existing theoretical explanations, unlike Strauss and Corbin's approach. Finally, in the future I want to extend and verify the findings using hypothetico-deductive methodologies, and this means a realist perspective is the most pragmatic approach for answering this research question. 


\title{
Chapter Four - Developing a classical
}

\author{
grounded theory
}

As the artist picks his way along, rejecting and accepting as he goes, certain patterns of enquiry emerge. His failures are as valuable as his successes: by misjudging one thing he conforms something else, even if at the time he does not know what that something else is.

Bridget Riley, http://www.op-art.co.uk/bridget-riley/ (retrieved 5 May 2014)

In this chapter, I will show how I followed classical grounded theory methodology. I begin by describing the researcher's role in qualitative research in general, and then consider researcher bias and theoretical sensitivity in the context of classical grounded theory. On the basis that researchers should be explicit about what they bring to the research question, I outline my experience as it relates to my research question. I follow this by detailing the processes I used to obtain and analyse data for this study. The chapter concludes with my personal reflections on the process of conducting this research project. I will follow the organisational process Egan (2002) describes to show how I approached my grounded theory research, although the steps are not as linear as depicted in the following figure. 


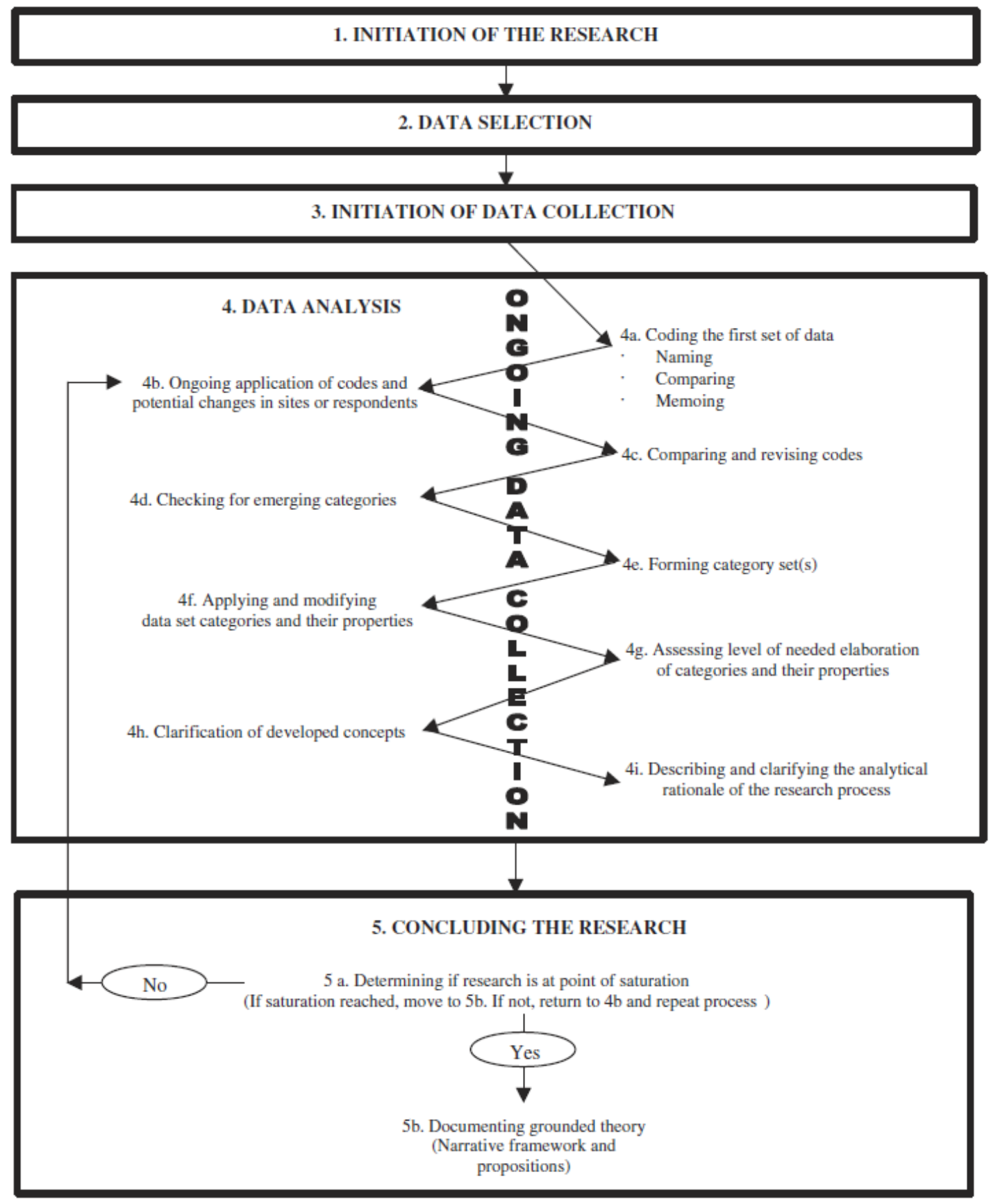

Figure 4. Grounded theory process (Egan, 2002) 


\section{Initiating research using grounded theory}

\section{Context and philosophy of science}

At the beginning of a qualitative study, researchers often state their personal preferences and describe the research context. This identifies some of the systematic biases that may influence the findings. Most pain research is carried out within a broadly positivist epistemology, and this stance implies that a researcher's decision-making, when selecting relevant variables and values in a research context, are unimportant. All research paradigms constrain what is accepted as reality, what can be used to determine "truth", and the degree of influence the researcher has on research findings (McGrath \& Johnson, 2003). What differs is the extent to which these are openly declared.

Quantitative researchers aim to determine the objective properties of phenomena independently of the viewpoint of the individual using carefully calibrated tools or instruments to maximize reliability. This means any researcher should be able to use the same instrument and methodology to arrive at a similar result. In qualitative research, the researcher is the research tool; thus personal knowledge, the effects of fatigue or lack of attention, and cognitive biases can influence data collection and analysis. While developing accurate and reliable measures, and instrumentation consume much time and energy in quantitative research; in qualitative methodologies, researchers spend comparable time developing their skills to collect and analyse data in ways that maximise trustworthiness.

Despite the apparent differences between qualitative and quantitative methodologies, both, as Henwood and Pidgeon (1992) point out, simplify and organise complex information. The epistemological differences between methodologies are arguably less relevant than how well-suited they are to answering the research question. Below, I describe my philosophical position, personal background, and clinical orientation to make 
these explicit and ensure readers are aware of any influence they may have had on my findings.

\section{Personal values, assumptions and biases}

I have worked for 20 years in the field of chronic pain, primarily as a member of an interdisciplinary team working within a cognitive behavioural approach. I developed an interest in how and why people manage their pain and their lives in different ways as a result of both professional and personal encounters. I have met many people who describe themselves as well despite having chronic illness, and so I have also become increasingly interested in exploring what contributes to resilience and recovery and in how this could be included within self-management for people with chronic pain. I am also keen to learn how people naïve to pain management manage their pain in daily life. From my clinical experience, I know that health is not simply the presence or absence of a disease process, it is a combination of biological, psychological and social factors (Engel, 1977).

Most chronic pain assessments focus on problems and skill deficits, with relatively little attention paid to what works well for the individual. In my experience, people rarely deliberately set out to use coping strategies that are unhelpful; rather, they adopt coping strategies that they believe will help them. Unfortunately, many use strategies that initially help, but over time, become less useful and may indeed be contributing to distress and disability. One example of this is the use of resting. In acute pain situations, resting gives damaged tissue an opportunity to repair, but when pain is chronic, resting may interfere with valued activities. Resting is associated with avoidance, passive coping, disability and both distress and depression (Brown \& Nicassio, 1987; McCracken \& Samuel, 2007a; Snow-Turek, Norris, \& Tan, 1996), yet it is a strategy often employed.

The cognitive behavioural approach to chronic pain management helps people reconceptualise their pain as something that can be managed and supports them to 
develop useful coping strategies so they can return to personally-relevant pursuits. I have provided this approach for chronic pain for most of my clinical career. People with chronic pain have told me, however, they have heard many different messages from health providers, and they often lack trust in their health providers (Peters et al., 2009). They describe feeling demoralised when many of the medical interventions used to reduce pain fail to eliminate it entirely (Turk et al., 2011). The process of seeking a cure, and coping with ongoing pain interferes with normal life (Eccleston \& Crombez, 2007). In contrast, others continue living well despite their pain, experiencing far less distress and disability. My belief is that clinicians can be able to learn from people who cope well, and thus may be able to broaden the range of what they offer to people looking for relief from distress and disability.

\section{Beliefs about people, coping with pain and pain management}

In clinical practice, I concur with Turk (2002) and Morley, Williams, and Hussain (2008) in believing that people actively process information rather than passively reacting to what occurs around them. It is clear to me that thoughts influence emotional and physiological arousal, and thus can motivate behaviour; emotions, physiology, and behaviour similarly affect thoughts and beliefs, while the environment and the individual influence behaviour, and in turn reciprocally influence what a person does (Turk \& Monarch, 2002). If people have learned unhelpful ways of thinking, feeling and behaving, then interventions designed to alter behaviour should also focus on unhelpful thoughts, feelings, physiological responses and behaviour (Flor \& Turk, 2011). If people actively develop and maintain unhelpful ways of thinking, feeling and behaving, then they can, are, and should be actively involved in changing their unhelpful ways of responding (Turk, 2002, p. 140). I also believe that people can alter their relationship to their pain to allow them a broader sense of identity beyond their pain. They can reconceptualise their identity to being a 'well' 
person with pain, and do so with learning or refining skills that help them deal effectively with either their pain, or the consequences of their pain (Morley, Shapiro, \& Biggs, 2004). People are adaptive and choose strategies that work, at least in the short term, and we can learn much about what works for managing pain if we listen to, and observe, people who cope well (Seligman \& Csikszentmihalyi, 2000; Zautra et al., 2010). Finally, I believe that participating in valued activities is a fundamental component of quality of life (Borell, Asaba, Rosenberg, Schult, \& Townsend, 2006; Fisher et al., 2007).

Effective analgesia has only been widely available since the mid-1860s. People at other times in history and even now, in many parts of the world have coped with painful conditions without pain relief, and many have done so with equanimity. I believe an undue focus on pain reduction and cure in this century may inadvertently interfere with participating in many valued activities (McCracken, Vowles, et al., 2007), and may even limit future ability to develop resilience in the face of adversity.

Finally, I believe that people with chronic pain deserve to be offered choices in how they approach managing their pain, and be able to choose strategies that align with their values. They should be offered the opportunity to live without depending on healthcare (Buenaver, McGuire, \& Haythornthwaite, 2006). In some instances, I think pursuing pain reduction produces dependence and increases suffering (Loeser, 2012).

Declaring my personal values and making them explicit has not been easy. Although this section of my thesis is part of "received" qualitative research method, it has also given me insight into the way I framed and shaped the research questions and chose my methodology.

\section{Philosophy of science}

In terms of philosophy of science, I draw on Manicas and Secord (1983) who propose a realist theory of science with the following premises: 
1. Knowledge is both culturally and historically contextual, and facts are "theoryIaden". While science seeks to develop theories to explain the real world, researchers carry out their work at a time, place and culture; this means explanations for phenomena are imperfect but are refined over time. Rational criteria are used to determine how well theories represent the real world because, according to the realist paradigm, the world exists independently of the knower (Manicas \& Secord, 1983).

2. The real world is complicated and "stratified". Each entity or phenomenon in the world is made up of other simpler units. What we learn at one level of analysis influences knowledge of an entity or phenomenon at another level (Manicas \& Secord, 1983).

3. Scientific laws describe causal properties of structures that exist and operate in the world. Within scientific realist philosophy, it is just as relevant to understand when a law does not hold true as it is to know when a law does operate. Scientific realists prefer tendencies and probabilities and likelihoods rather than absolutes when testing hypotheses (House, 1991). Knowledge of the real world can, therefore, only be an approximation because there is always the possibility of intervening variables or additional layers of complexity, thus the process of discovery and experimentation can only progress stepwise towards an understanding of what exists in the real world (House, 1991).

4. Theory provides causal explanations to show how, in a given situation, a relationship occurred to achieve a result. In order to explain how something happened, researchers must analyse the phenomenon into its parts and describe how these parts relate to one another. To do this requires working backwards from the event to all potential causes, then eliminating alternatives that might have worked their way into the situation at the time. This form of reasoning is abductive, or inference to the best explanation (Harman, 1965). 
Researchers using classical grounded theory methodology may adhere to any philosophy of science. Classical grounded theory methodology examines what occurs in the real world (it is grounded); social and historical realities form part of the context. Extant theories earn their way into the emerging theory by extending or clarifying relationships, or explaining phenomena (incidents) provided by the participants. Grounded theories can be modified as new data appears. Thus, scientific realist assumptions can be accommodated within classical grounded theory methodology.

\section{Ethical issues}

In New Zealand, there are explicit requirements for researchers to ensure they adhere to the principles of ethical research. Those principles are:

- Respect for people, and for their rights, incorporating the principles of autonomy and protection of people with impaired autonomy.

- Respect for, and a commitment to the principles of the Treaty of Waitangi demonstrated by partnership, participation and protection of Maori rights and interests, particularly recognizing how results of research may impact upon Maori.

- Ensure that research is just, fair and provides a balance of "burdens and benefits".

- Make certain that any risks to participants are reasonable in light of any expected benefits, and in general, protecting the rights of participants.

- Demonstrate integrity in the way in which research is conducted; being honest, thoughtful, rigorous and accountable for research activity.

- Ensure a diverse range of participants is included as far as possible, and to avoid discrimination on grounds unrelated to the research aims. 
- Minimise any conflicts of interest. (National Ethics Advisory Committee, 2012, pp 8-10)

The University of Canterbury also reviews the ethical conduct of any research requiring human participants. I needed to meet the requirements of both committees as described below and have included copies of relevant documents in the Appendix.

I consulted with Tahu Potiki Sterling of Nga Ratonga Hauora Māori/Māori Health Services and Chairman of Te Komiti Whakarite, the CDHB Research Consultation with Maori Committee. Mr Stirling supported my research and offered to meet with me and help to recruit participants as needed. This did not prove necessary.

I developed a participant information sheet, outlining the research aims, process and steps taken to protect the confidentiality of personal information obtained as part of the study (these documents are located in Appendix 1). I thought it particularly crucial to indicate that if, as a registered health professional, I felt any participant was distressed or disclosed severe untreated mental health or substance abuse issues; I would seek permission to contact the participant's usual doctor. I offered participants at least 24 hours, often more, to think about their participation; the opportunity to use an interpreter if required, and to conduct the interview at their choice of venue (either the participant's own home, or at my office in the School of Medicine, Christchurch Hospital. Some participants preferred to use Skype for their interview). I advised participants that they could withdraw from the study at any time, without prejudice.

Each participant signed a consent form. This included consent to contact their usual doctor if needed (either at the participant's request, or at mine); permission to record the interview using either digital audio alone, or video (Skype) recording; and permission to collect and retain their information for the purposes of this study. I also obtained permission to contact participants at a later stage if required (e.g. to get additional data). 
Steps were taken to ensure privacy and confidentiality of all information. I stored consent forms in a locked cabinet, in my office. I saved recordings on password-protected computer files on two memory sticks. The memory sticks were stored, in separate locations, to protect against data corruption or loss. I used code numbers for all participants and saved the files using the same code number. During initial coding, I used code numbers to identify participants. Participants' questionnaires were de-identified and saved in electronic files.

I sought approval from the University of Canterbury Human Ethics Committee, and the Upper South B Regional Ethics Committee of the Ministry of Health URB/11/02/009, and was granted this.

\section{Data selection and collection}

Although data collection and analysis proceed concurrently in grounded theory, to begin with researchers need to decide on the "location and identification of potential data sources associated with the research question" (Egan, 2002, p. 282). Beyond this starting point, subsequent questions and participant characteristics cannot be pre-determined. Comprehensively reviewing the literature prior to beginning the study may constrain the study by forcing the data or biasing the researcher (Glaser, 1978, 1998). Decisions about participants' characteristics and questions to ask must be developed in response to the need to clarify aspects of the emerging theory. Participants must be actively engaged in the sphere of action, but the usual restrictions of ensuring representation of different ethnic groups, ages, and from both genders do not apply. Instead, researchers select participants based on how they might provide interesting and material variations of relevant incidents.

I wanted to learn about people who had not been exposed to a cognitive behavioural approach to managing pain, and I needed to ensure they believed they were living well 
despite their pain. I also wanted to rule out some of the complex issues that can make it difficult to interpret pain and coping research, and so I needed to establish some boundaries for my study (Patton, 1980).

\section{Establishing the study boundaries}

I decided early in the study to limit participant recruitment at least initially to people with painful rheumatological conditions. I eventually included people who had chronic pain with less clear-cut aetiology and treatment, but to simplify the inclusion criteria and eliminate some of the confounds relevant to wellbeing such as diagnostic uncertainty (Mishel, 1999), I initially sought participants with ankylosing spondylitis or rheumatoid arthritis. I chose not to include participants with injury-related pain because compensation processes add complexities to pain and disability (Chibnall \& Tait, 2009).

I excluded participants who had attended a pain management programme, or who had seen a clinical psychologist or occupational therapist for managing their chronic pain. A principal aim of this study was to establish how people, who were naïve to "traditional" cognitive behavioural approaches to pain management, managed their pain. I also excluded participants using regular opioid analgesia for their pain, with the exception of medications used for managing the inflammatory factors in rheumatological disease. I incorporated this limitation to ensure participants regularly experienced moderate to severe levels of pain and thus needed to use a broad range of active coping strategies.

I asked that participants identified themselves as "living well" despite having chronic pain. This requirement captured an essential element of resilience and wellbeing. All participants were in full-time paid or unpaid work or study although I did not indicate that this was a requirement for the study.

I did not place any requirements for age, gender, ethnicity or comorbid health conditions. I obtained consent from seventeen participants ranging in age from 19 - 65 years; including 
two people who identified as Maori (one Ngai Tahu and one Ngati Porou); working in a range of occupations including professional, technical, semi-skilled, and self-employed; and an equal number of males and females took part. None of these demographic variables emerged as relevant to the theory.

\section{What is good grounded theory data?}

The quality of any research depends on the quality of the data. I needed to obtain data from individuals who identified themselves as "living well with chronic pain". I needed to find firstly their main concern, and then enquire about the ways they resolved it. Very quickly I found that their main concern was about resolving the threat to self-coherence posed by chronic pain, although participants used the words "how to get on with life". The core category and subsequent categories needed to be related how participants resolved their main concern, which ultimately explains the process of re-occupying self. Information I collected needed to pattern out, in other words, for categories to be saturated. Saturation means that the research can identify no new properties or dimensions of a category despite obtaining more incidents that could code to that category. Properties identify features or characteristics of a category, and can be a dimension (larger, smaller), a quality (stabbing, blunt, hot), or another characteristic such as shape, speed, or complexity. Some categories are sub-categories of a higher conceptual category, in which case the sub-category conceptualises a property of a higher order category.

I knew that grounded theorists can obtain four types of data:

- Baseline data: "the best description a participant can offer" ;

- Properline data: "which is what the participant thinks it is proper to tell the researcher" ; 
- Interpreted data: "what is said by a trained professional whose job it is to make sure that others see the data his [sic] professional way" ; and

- Vaguing out: where "there is no stake in the participant in telling the researcher anything, so he just vagues out" (Glaser, 1998, p. 9).

I had to ensure I collected minimal properline and interpreted data, and I needed to guide the interviews to minimise vaguing out. Once I asked how participants learned to get on with it and live well, collecting baseline data became relatively easy because this question elicited free-flowing descriptions of the ways they resolved the problem of chronic pain in their lives. When interviewing people who interacted with those who cope well, such as family members and health professionals, I was collecting interpreted data, so I delayed these discussions until later in my study, and like all other data, concepts within these responses needed to pattern out to work into the emerging theory. By following these steps I claim I have obtained high-quality data from people in the substantive area, talking about their main concern and how they have resolved it, thus satisfying one of the Rey quality criteria for good grounded theory.

\section{Data collection strategies}

\section{INTERVIEWING}

Interviewing is a major approach to data gathering in qualitative research. Interviews provide a rich source of data and can capture some of the complex and multi-layered human experience (Polkinghorne, 2005), are flexible (Cohen, Manion, \& Morrison, 2011), require little in the way of technology or equipment, and are relatively uncomplicated to conduct. Gillham (2000) describes the interview as "a managed verbal exchange", but Polkinghorne makes the point that helping a research participant to disclose a "full and detailed account" requires considerable skill (Polkinghorne, 2005). Interviews have limitations not only because of interviewer technique, but also because participants may 
self-censor their responses or recall only superficial details (Steblay, 1997); they may be influenced by the interview process (Denscombe, 2007); and, because language itself is a symbol used to represent experience there is always opportunity for misunderstanding (Bickerton, 2003).

While interviews are not the only approach to data collection in classical grounded theory, I chose interviewing because it is flexible, and I hoped it would enable me to probe more deeply to understand constructs as they emerged. It is also a skill I have used extensively in my clinical work. Research interviewing and clinical interviewing are both designed to understand the individual's perspective and experience. Implicit or explicit theoretical and philosophical perspectives influence the content of both forms of interview; both require empathy; and for the researcher or clinician, the ability to guide the participant to provide relevant information. At heart, however, the clinical interviewer is there for the individual, while research interviews meet the researcher's needs (Hunt, Chan, \& Mehta, 2011). This means the "power relationship" between interviewer and interviewee is different from a clinical situation, and I needed to remain aware that I was a temporary visitor in this person's life. To acknowledge this, I gave participants, where possible, a small koha (gift) in appreciation for their time and willingness to talk to me.

I followed a semi-structured interview approach, and each interview took between 50 130 minutes. I contacted two participants a second time to elaborate on particular aspects of their experience, thus providing additional data for the emerging theory. For the first few interviews, I started with a semi-structured framework developed from Charmaz (2006b) included in Appendix 2. I quickly learned to use this structure very loosely to allow time to explore aspects relevant to the theoretical explanations, particularly as interviewing and coding progressed, and theoretical categories began to emerge. Glaser does not support the use of structured or semi-structured interviews, believing that this can force or pre-determine what the participants describe (Glaser, 1998). I decided, 
however, to use the headings within a semi-structured interview to mitigate against some of the cognitive biases that influence human decision-making, such as recency bias, confirmation bias and selective perception (Ruscio; Schwab, 2008). This is the same interviewing strategy I use in my clinical work and follows the principles described by Vertue and Haig (2008). I reviewed the interview domains after each meeting and noted any new areas to include or eliminate. As I prepared for the next person, I again reviewed the interview schedule, and read notes and memos made during coding, to identify any necessary changes. Thus, data collection proceeded in an iterative fashion.

I decided to record interviews for later upload into NVivo. This ensured I did not omit any interview material, and maximised the value both of the data and of the software (Bringer, Johnstone, \& Brackenridge, 2004; Wiltshier, 2011). I wrote notes after each interview and then imported both the notes and the recording into NVivo. I then listened to, completed gisted transcriptions, and coded the interviews directly into NVivo. This preserved the spirit of interviewing as described by Glaser (Glaser, 1998), and also allowed me to listen to the interview in total, making the context of incidents described by participants more apparent. It also meant I could save all files, including recordings, memos and codes together thus maintaining data security.

\section{QUESTIONNAIRES}

In addition to interviewing, I incorporated standard psychometric tools used in pain research. Questionnaires are developed from extant theory, and while there is a risk of imposing a theoretical perspective on an emerging theory, questionnaire data can be used as simply another piece of data to inform the developing theory (Glaser, 1998). I coded questionnaire responses as I did constructs from the literature, and the interview data. Constructs had to earn their way into the emerging theory, and questionnaire responses provided another source of such constructs. While I have critiqued the basis of coping questionnaire development in Chapter two, they provide useful normative data that allows 
comparison between participants responses and those of known populations. In this project, they were also used to enable participants to identify coping strategies from a list, which may have helped them to remember strategies they might not have done so spontaneously. I gave participants several questionnaires after their interview (to minimise priming participant recall) and asked them to return the questionnaires within one week of their interview. The questionnaires I used were:

a. The Pain Coping Profile: Short form (PCP:S) The PCP:S is a 17-item questionnaire used to assess the nature of a respondent's pain experience over the previous 6 months in terms of pain severity, pain-related functional interference, and emotional burden (RuehIman, Karoly, Newton, \& Aitken, 2005). It has been used to identify resilient individuals from a general population of people with chronic pain (Karoly \& Ruehlman, 2006) and in a study in primary care (Karoly, RuehIman, Aiken, Todd, \& Newton, 2006). Test-retest reliability, internal consistency, factor structure, and social desirability bias are all acceptable. I used this measure to explore aspects of pain intensity and interference amongst participants and helped to confirm that they had similar characteristics to those in Karoly and RuehIman's (2006) study.

b. Chronic Pain Acceptance Questionnaire (CPAQ) and Pain and Activities Relations Questionnaire (PARQ) were developed by McCracken and colleagues to study the place of acceptance and the relative activity levels of people experiencing persistent pain (McCracken, Vowles, \& Eccleston, 2004). Both acceptance and activity approach/avoidance are considered essential constructs in adjusting to persistent pain, and activity levels undertaken despite pain (McCracken \& ZhaoO'Brien, 2010). As these are relevant to the resilience construct and coping with chronic pain, I included them in the study. Satisfactory psychometric properties for 
both these measures have been established (McCracken, MacKichan, $\&$ Eccleston, 2007).

c. The final questionnaire used was a measure of many coping strategies introduced to people with chronic pain during cognitive behavioural pain management programmes. The Chronic Pain Coping Inventory (CPCI) is often used as an outcome measure in pain management research. Developed by Jensen and colleagues originally in 1995, it has acceptable psychometric properties, but I amended the scoring slightly to accommodate the anticipated lower rates of use. I asked participants to identify how often they used the listed strategies over the past month using the following scale: $0=$ never, $1=$ less than once a month, 2 = once a month, 3 = once every couple of weeks, 4 = once or twice a week, 5 = once a day, 6 = more than once a day. The original asks participants to identify how many days they used the strategies over the past week, ranging from 0 - 7 (Jensen et al., 1995). The literature on the CPCI provides a body of data on the patterns of responses from people before and after treatment and on those with high and low disability.

\section{Participants and recruitment}

Sampling in a grounded theory study begins wherever people are engaged in solving their main concern (Glaser, 1998, p 117, 123). For me this meant finding people with painful conditions who thought of themselves as living well and coping with their pain. One of the first questions people have asked me when I describe my research, is "how do you find these people?" People who cope well with chronic pain may not be found in doctor's rooms or pain management services, and I initially thought I would find participants at gyms, fitness centres, swimming pools and recreational clubs and service organisations. To complicate matters, I obtained ethical approval for this research in January 2011, one month before the tragic February 2011 Canterbury earthquakes. With most of the sports 
centres and community facilities closed for more than six months (and many have since been completely demolished), and residents moving within and away from Christchurch, I needed to use alternative strategies to locate and interview people.

Arthritis New Zealand is a national organisation providing education, advocacy and support for people with rheumatic conditions where many people with painful conditions go to seek advice. I have been a speaker at several educational meetings open to members of the organisation and the general public, and obtained permission to ask for participants at two meetings during late 2011.

New Zealand is a connected nation, with a highly technologically-literate population (Labour, 2008). I placed recruitment notices on two Facebook pages for New Zealanders with rheumatological conditions and pain, and also put notices on my personal blog "Healthskills" (http://healthskills.co.nz). As a blog author since 2007, I am known in some circles on Facebook, and on my blog and recruited three participants this way. I also follow pain-related media releases and contacted a further three participants on the basis of articles in the media. As a result of widening my recruitment strategy, some of the participants did not live in Christchurch, and for these people I used Skype as the interview platform.

Once I received an enquiry from people interested in my research, I approached the individuals via telephone or email and asked if they would be interested in participating in my study. After sending them some brief information about the study, I did not approach them again but waited for them to contact me, and in each case, they did and asked for more information. I then forwarded the Information sheet and Consent form, and asked them to complete and return them. I knew three individuals personally and followed the same approach as above. No prospective participant declined to be part of my research, and I excluded only one potential participant. This person had previously participated in a 
self-management programme, and I excluded the individual because I wanted to explore the coping strategies of people who were naïve to pain management.

In the latter stages of data collection, I also approached people who were not associated in any way with Arthritis New Zealand: I encountered these people when I spoke about my research topic in daily conversations, via my blog (www.healthskills.co.nz), through a Facebook group "Super Young Arthritics of New Zealand", and I also purposefully identified those with less well-defined pain problems.

\section{Sampling}

Grounded theory employs purposive sampling and theoretical sampling or "grounded deductions, feeding into data for more induction as the growing theory leads the research on" (Glaser, 1998, p. 157). The emerging theory directs both questions and participant selection as the researcher delimits each category to include only those constructs relevant to the core category. Delimiting involves gradually reducing the number of categories and incidents by subsuming them into more inclusive categories or eliminating those that have limited relevance to the core category. By doing this, the developing theory articulates how each category and property relate to the core variable (Glaser \& Holton, 2007b).

Delimiting and theoretical sampling began to occur once the first category was saturated (saturation is when no new properties of a category are identified while coding (Glaser $\&$ Holton, 2007b). I selected participants and the data collected from participants, to elaborate or clarify particular concepts. This meant I refined my inclusion criteria. For example, I initially chose people with specific rheumatic diagnoses but later identified those with less clear-cut diagnoses. I also revised the topic areas I included in interviews. My questions became more specific as I sharpened my focus on identifying the properties of particular categories. My list of codes was initially extensive, but over time codes were incorporated within categories as I recognised incidents provided by participants were 
reflecting the properties of an underlying category. I conducted 17 interviews, returned to two participants for further interviews, and the final interview did not produce any new codes; thus I believed that saturation had occurred and completed my data collection. Demographic details and questionnaire scores are located in Appendix 3.

\section{Data management}

\section{USING NVIVO}

As critical to successful research as data collection, data management is an essential, albeit unglamorous, part of the process. Qualitative research generates a wealth of data and often in formats that are not easy to manage. I used field notes, questionnaires, data sheets (face sheets), audio and video recordings, emails and diagrams, all of which needed to be managed to protect participants' confidentiality, data integrity and for analysis. I allocated each participant a code name and linked each file to the relevant code name within NVivo. NVivo 9 is a qualitative data management programme developed by QSR International and developed from NUD*IST, one of the first qualitative data management software programmes. NVivo 9 has numerous features including graphical displays, text analysis, and the ability to link and sort files in different formats such as text, image, sound and video. I developed data sheets to collect participant biographical details including contact details, and saved these as Adobe pdf documents so they could not be readily altered. I saved these documents to a password protected file and retained them in two separate secure locations. I emailed questionnaires to participants, and once they were returned, scanned and saved them as password protected pdf documents. They were then imported to NVivo 9. I wrote field notes and memos directly into NVivo, referring to notes I wrote immediately after each interview. I saved audio and video recordings, emails and drawings in password-protected folders and imported them into NVivo 9. I used NVivo to code interviews, visualise data and manage both the confidentiality requirements and the need to locate and use data within a single platform. The NVivo interface enables 
researchers to view the original recordings and notes, and use codes from other recordings and field notes from within the software.

I completed a gisted transcription for each recording, summarising the essential content of each interview (Evers, 2010). I did this to develop an overview of the interview, and it also gave me an aide memoir when I returned to the recording to look for specific incidents relating to a category as the theory emerged. After finishing the gisted transcript, I completed the first coding pass through the interview, simultaneously generating codes and notes about each code as I worked. As I progressed with the interviews, I compared each incident with existing incidents and codes, using the constant comparative method and asking the questions "what category does this incident indicate?"; "what property of what category does this incident indicate?" and "what is the participant's main concern?" (Glaser, 1998, p. 140). I often returned to earlier interviews to review incidents as I selectively coded to identify properties of a category.

There is a long practice of transcribing interviews in qualitative research although this is rejected by both Glaser and Strauss, saying that it can lead to descriptions rather than conceptualisation because of data overload from having too many details to conceptualise (Glaser, 1998; Glaser \& Strauss, 1965). Evers (2010) considers that transcribing enhances analytic quality because transcriptions increase accuracy and provide greater awareness of the interview content, but I chose not to use verbatim transcription to follow Glaser's process more closely. I used audio or video recordings to allow me to focus on interacting during the interview rather than dividing my attention between asking questions, listening to answers and taking notes. I coded, however, directly from the recordings, using the gisted transcription to locate relevant incidents.

\section{WRITING MEMOS}

An integral part of grounded theory involves recording thoughts and responses to data and coding in the form of memos. Memos capture the reasoning used as coding 
proceeds, helps to catch fleeting thoughts about the data including questions that need to be considered in the next interview, record musings on extant literature, and capture ways in which the categories might relate to one another. Writing memos is the primary way to record ideas about substantive codes and theoretical relationships; writing memos also generates leads for further inquiry during theoretical coding. Memos provide the basis for writing up the completed grounded theory. The theory is finally integrated by sorting completed memos; the key question for each memo during sorting being "where does it fit in?" (Glaser, 1978, p. 123). Each memo is challenged, questioned and compared with each idea within the emerging theory, generating more memos which are also sorted into the theory. I wrote over 200 memos and stored many of these within NVivo. I produced further memos while writing this thesis, although as the model had developed to a smaller number of categories I used MS One Note on my computer desktop, beside the open Word document. While Glaser rejects using computer assistance for grounded theory, I found I could use NVivo, One Note and Word as tools in the service of grounded theory analysis rather than to "muscle the analysis" (Silver \& Patashnick, 2011; Thomas, 2011). Being able to visualise relationships between categories and memos and creating tentative models without copying or physically cutting documents worked well for me.

\section{A NOTE ABOUT NVIVO AND SIMILAR COMPUTERISED QUALITATIVE DATA}

\section{MANAGEMENT TOOLS:}

NVivo offers a convenient way to manage, save and code data, and also has numerous data analytic tools such as graphs, concept mapping and models. After several interviews, I was tempted to use some of the tools within NVivo to explore the relationships between codes. This was unhelpful. There were too many codes, the relationships illustrated by NVivo developed from how often they appeared in the data rather than from conceptual relevance, and I tried to impose my thoughts on what was happening rather than allowing it to emerge through constant comparison. A screenshot of one of my attempts will 
illustrate the complexity (Figure 5).

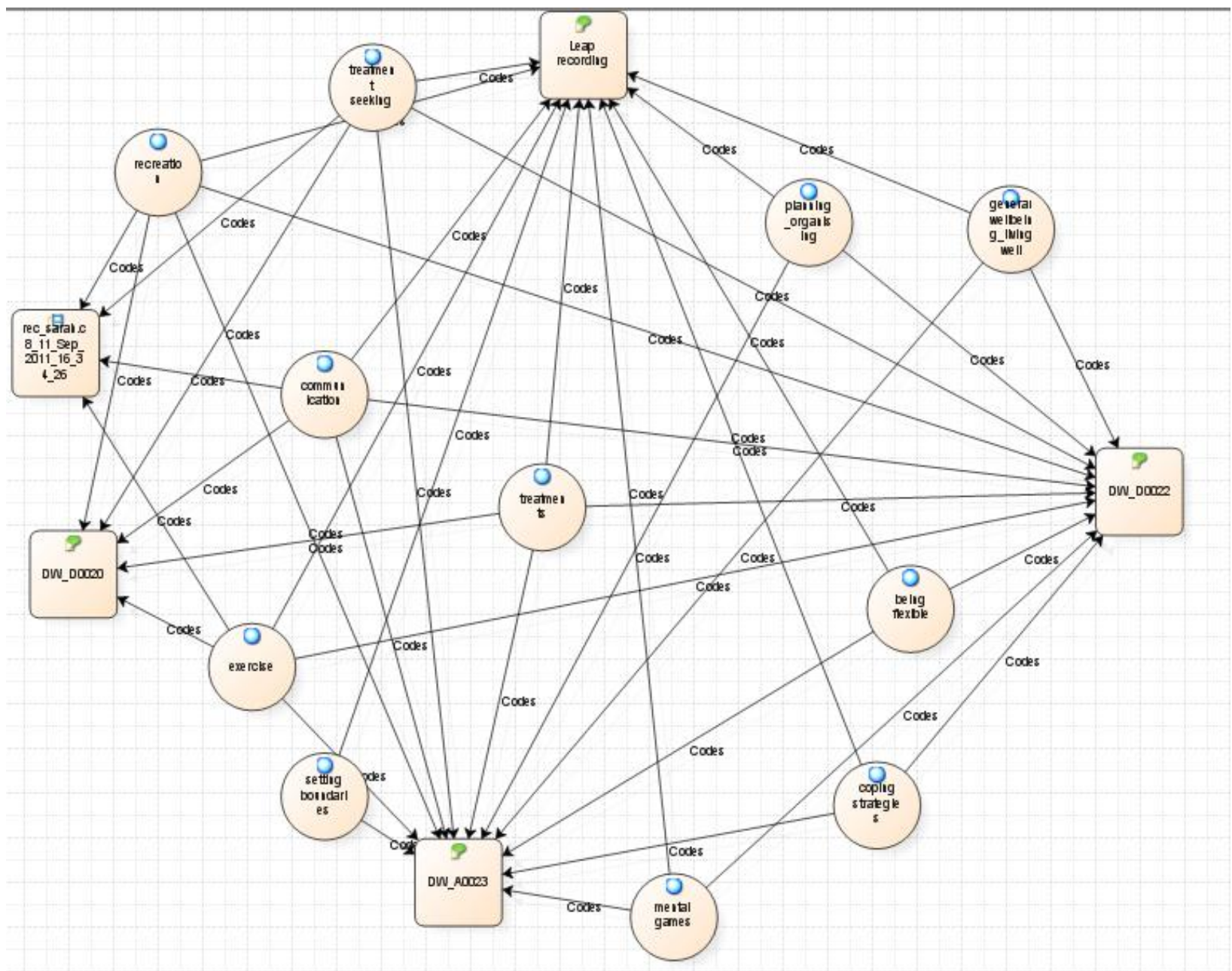

Figure 5. Example of NVivo analysis tool

I found the models generated within NVivo failed to capture meaningful relationships based on emergent concepts. Other grounded theorists have described successfully using NVivo to generate models (Bringer, Johnston, \& Brackenridge, 2006; Silver \& Patashnick, 2011) thus my lack of success may have been due lack of experience with the software. I used the software simply as a tool to aid in data management rather than for any analysis. 


\section{Data analysis}

Data analysis in grounded theory proceeds in an iterative way beginning with open coding in which every incident is given one or more labels, often derived from words uttered by participants. Each incident is compared with other incidents using the constant comparative process and coded to capture, as Glaser says, "what is going on" (Glaser, 1998, p. 123). An important part of the analysis is discovering the core category which is related to all other categories and explains how participant's main concern is resolved. Participants and the areas to interview are selected on the basis of obtaining data to saturate both the number of categories relevant to the core, and the properties of each category. By collecting further examples of incidents that describe properties of the category, and also beginning to delineate relationships between categories, a grounded theory actually is grounded by abductively reasoning from specifics to generalities. In this section, I want to discuss two aspects of my data analysis process. The first is coding, and how the relatively unstructured approach advocated in classical grounded theory is carried out. I then turn to how I went about integrating existing theoretical and experimental findings from pain research.

\section{Coding}

"Any researcher who wishes to become proficient at doing qualitative analysis must learn to code well and easily. The excellence of the research rests in large part on the excellence of the coding" (Strauss, 1987, p.27).

Coding is a broad term used widely in qualitative research. It refers to a range of methods used to reduce the volume of detail accumulated during the course of data collection. Saldaña (2013) defines coding as "a word or short phrase that symbolically assigns a summative, salient, essence-capturing, and/or evocative attribute for a portion of language-based or visual data" (p. 3). 
A common criticism levelled at classical grounded theory is that the process of coding lacks structure or concrete steps for each stage (Backman \& Kyngäs, 1999; Huehls, 2005). Strauss and Corbin (1994) wrote their book to explicate the process for new researchers using the methodology. They originally described a highly structured process of open coding where each word or phrase is coded with one or more unique codes; axial coding in which the conditions, actions and consequences of the phenomenon and actions under study are defined; and finally, selective coding where all other categories are related to both the core category and one another. The theory is integrated and refined by the end of selective coding (Strauss \& Corbin, 1994; Walker \& Myrick, 2006a, p. 556).

Classical grounded theorists use three forms of coding: open coding, selective coding and theoretical coding. Selective coding, as used within classical grounded theory, involves coding only for categories directly relevant to the core category, and occurs only once the core category has been identified. Theoretical coding occurs during sorting and writing up. Glaser has repeatedly argued that Strauss and Corbin stray from the original grounded theory methodology and calls the product of research conducted using their processes "full conceptual description" rather than a latent multivariate theory. Glaser further contends that Strauss and Corbin force theoretical relationships on the data by including extant literature during open coding (i.e. at the beginning of analysis) and by their use of axial coding and the conditional matrix (Glaser, 1998, 2003; Glaser, 2008; Glaser \& Holton, 2007b). Corbin and Strauss have changed their original stance, and Corbin now indicates that the procedures they had advocated in their original book, can be used more flexibly and are simply tools for use by researchers (Corbin \& Strauss, 2008a). In choosing classical grounded theory, I followed Glaser's approach and found the process both intense and liberating. 
To begin coding, the researcher first labels each incident with as many codes as possible and then compares every incident with other incidents with the same code. This is the fundamental process of constant comparison used in classical grounded theory.

Once there are enough incidents to identify the underlying pattern described by those incidents, the code can be defined as a category. The researcher's aim is to discover interchangeable indicators (individual incidents) that detail the properties of each category in a form of latent structural analysis (Glaser, 2011). Codes, categories and concepts are interchangeable terms used to indicate latent variables (Glaser, 2011).

Coding begins with open coding and moves to substantive coding once the core category and main concern are identified. Selective coding occurs during purposive sampling, when only those incidents needed to develop categories are identified and coded. Theoretical coding forms the final part of classical grounded theory and is used when writing and sorting memos (Glaser, 1978, 1998, 2005; Kelle, 2007a).

In my initial interviews, I used open coding to generate a large number of categories. I later reduced these by subsuming codes I had used for only a single incident within more abstract or conceptual categories. After I had completed seven interviews, I began selective coding because I had identified my core category, although I had not finalised the name. From then I coded only those incidents that related to the core category by carefully using constant comparison. Selective coding involves defining or delimiting the properties and relationships of existing categories and the core category. In practical terms, this was where I began to focus my interview questioning on particular aspects of categories I wanted to understand in more detail.

I initially felt overwhelmed when coding because I could see, after the first four interviews, no relationship between the codes. I can readily identify with the desire to impose an external structure on the data, and was especially tempted to draw from my clinical knowledge. I was able to identify the main concern of my participants by persisting with 
constant comparison. With this discovery, finding the core category was far easier. The number of other categories reduced as I delimited them to only those relating to the core while continuing to code incidents to detail properties of these categories.

\section{Integrating the extant pain literature}

While one purpose of a doctorate is to demonstrate the candidate's ability to develop work that makes a unique contribution to knowledge, at the same time no piece of work stands alone. Glaser, and indeed all other grounded theorists, recognise that although the grounded theory sits within a substantive area, the researcher's sensitivity to theory during theoretical coding, and while sorting and writing will add richness and depth to the theory, provide schematic frameworks for explaining the relationships between categories, and position the unique discovery of the grounded theory within the context of other findings (Charmaz, 2006b; Glaser, 1998, p. 74; Strauss \& Corbin, 1997).

As noted earlier, this study focuses on how people who identify that they live well with pain manage to do so, because I believe that there is much to learn much from those who are resilient. I also integrate material from psychology such as cognitive behavioural therapy, social behaviour, and communication, and from occupational science. These domains of knowledge reflect my personal interest and professional education and background, and I used them to inform and develop my theory.

There are several ways to integrate other research findings into an emergent grounded theory. The first is as contextual knowledge that helps to establish the boundaries of a particular study. While grounded theorists must avoid forcing pre-existing theoretical models on the data, there is no doubt that prior knowledge contributes to forming the research question and sensitises the researcher to certain patterns in the data (Charmaz, 2006b; Glaser, 1998; Strauss \& Corbin, 1994). My experience working in the field of chronic pain management and also teaching pain management to postgraduate clinicians means I know of cognitive behavioural approaches to managing pain such as Hanlon, 
Turk, and Rudy (1987) and Vowles, McCracken, and Eccleston (2007a); as well as psychological constructs such as fear-avoidance (Vlaeyen \& Linton, 2000); coping (Lazarus \& Folkman, 1984a; Tennen, Affleck, Armeli, \& Carney, 2000; Turner, Clancy, \& Vitaliano, 1987); catastrophising (Borsbo, Peolsson, \& Gerdle, 2009; Sullivan et al., 2001); and pain behaviour (Asghari \& Nicholas, 2001; Fordyce, 1982a).

Although I know I am sensitive to these concepts in view of inherent human cognitive biases (Ruscio, 2010; Schwab, 2008), I cannot point to exactly how this will have influenced the patterns I have detected in the information from participants. Biases occur in every piece of research, irrespective of philosophical orientation, and determine the way the researcher poses the question, the choice of methodology and the way participants are selected. I can merely indicate to readers that this will have occurred and indicate the concepts I know have informed my model.

Another way existing research findings are integrated is while naming codes during open coding and later, more explicitly, during theoretical coding. When open coding I used brief phrases participants used as the names for codes. Later, as I integrated codes, I gave more concise and descriptive names, some of which were derived from existing literature. For example, the category important occupations is derived from both occupational therapy and disability research(Ownsworth, Hawkes, Steginga, Walker, \& Shum, 2009; Pentland et al., 2003; Persson, Eklund, \& Isacsson, 1999; Pierce, 2001). During theoretical coding, as each category became more clearly defined, and relationships between various categories became apparent, I reviewed the literature relevant to the concept embodied in the category. I compared what has already been discovered with the results from my study, and incorporated those findings that "earned" their way into the theory. Finally, I referred to the literature that contrasts with my model as I explain how my findings provide a unique explanation for how people with chronic pain live well. 


\section{Developing a theory of living well with chronic pain}

The next section of this chapter details the process of developing the theory that has emerged from my research. It demonstrates the ways in which I have tried to follow classical grounded theory methodology in a "minus-mentor" situation and shows how challenging this work can be. I hope it also shows how rewarding the process is, and how, by being rigorous when following Glaser's approach, the results work, fit, and have relevance.

\section{First thoughts}

I initially thought this study would identify a number of coping strategies used in everyday life by individuals who coped well. I wondered whether these would be similar to those recommended in pain management programmes. I was curious to find out how people naïve to a cognitive behavioural approach to chronic pain might have developed these strategies. My focus was on what and how people used coping strategies, reflecting my clinical experience and readings.

In grounded theory, and as I quickly found out, preconceptions fail to allow patterns in the data to emerge without forcing the concepts to fit them (Glaser, 1992). I needed to put my assumptions to one side, integrating them only once I followed where the data led.

Grounded theory is "not logical, it is empirical, that is it seeks to find out what is going on" (Glaser, 1998, p. 91) and the methods, used consistently and honestly, allow the researcher to use "induction from the meanings of participant's experiences in a substantive area and therefore it excludes deductions or inferences of what should or might be going on, and includes deductions about where to further collect data on what is going on" (Glaser, 1998, p. 95). I changed my approach and started listening to participants describe their efforts to get on with life. 


\section{Open coding}

After each interview, I waited a day or two before reviewing the recording to allow time for recency bias to subside a little, thus reducing the risk of "hearing what I wanted to hear". I then listened to the recording as a whole and completed a gisted transcript.

I began coding from the beginning of each recording, pausing after I identified each incident, using constant comparison and writing the code into NVivo. At the end of coding each interview, I mind mapped the concepts and links. I also coded individual statements from the participant's questionnaires, as well as noting the scores they had obtained in domains measured by the questionnaires. Figure 5 is an early example of open coding and incidents associated with these codes.

During open coding, while I quickly found a way to "instil the spill" I had more trouble identifying the core category. As I collected more data, I subsumed the code "personal attributes" under the category "getting on with it" as I found that what I had coded as attributes or characteristics of individuals were mainly actions undertaken by participants. These actions were aspects of "getting on with it", while "getting on with it" related to, and explained, the greatest amount of variation in behaviour undertaken by individuals. At the time of the above coding example, I had not yet discovered this, and I was initially misled by what I assumed at the time would be significant.

At this early stage of data collection and analysis, I was unclear about how these codes related to one another, and the memo in Figure 6 illustrates some options I considered at that time (Figure 6). My comment "where does "I'm not doing myself any damage" come into it?" shows a question I posed which then informed my interviews until I could identify a consistent pattern to resolve my question. Interviews conducted at this time examined how participants learned about their diagnosis and how this knowledge influence their subsequent actions. Memos are a valuable way to document fleeting thoughts, possible relationships, areas to explore and ideas from reference reading. The importance of writing 
memos lies not only in ensuring ideas are not forgotten, but also in shaping interviews, and in providing the basis for sorting and integrating the categories in the final stages of theory development.
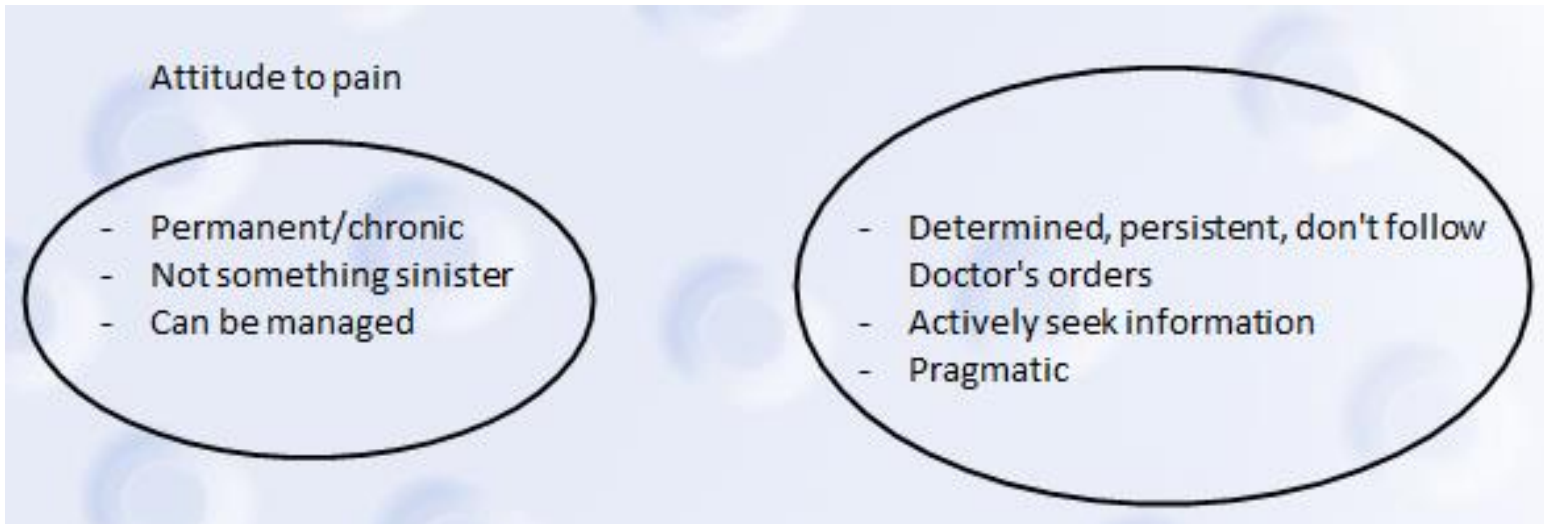

Personal attributes
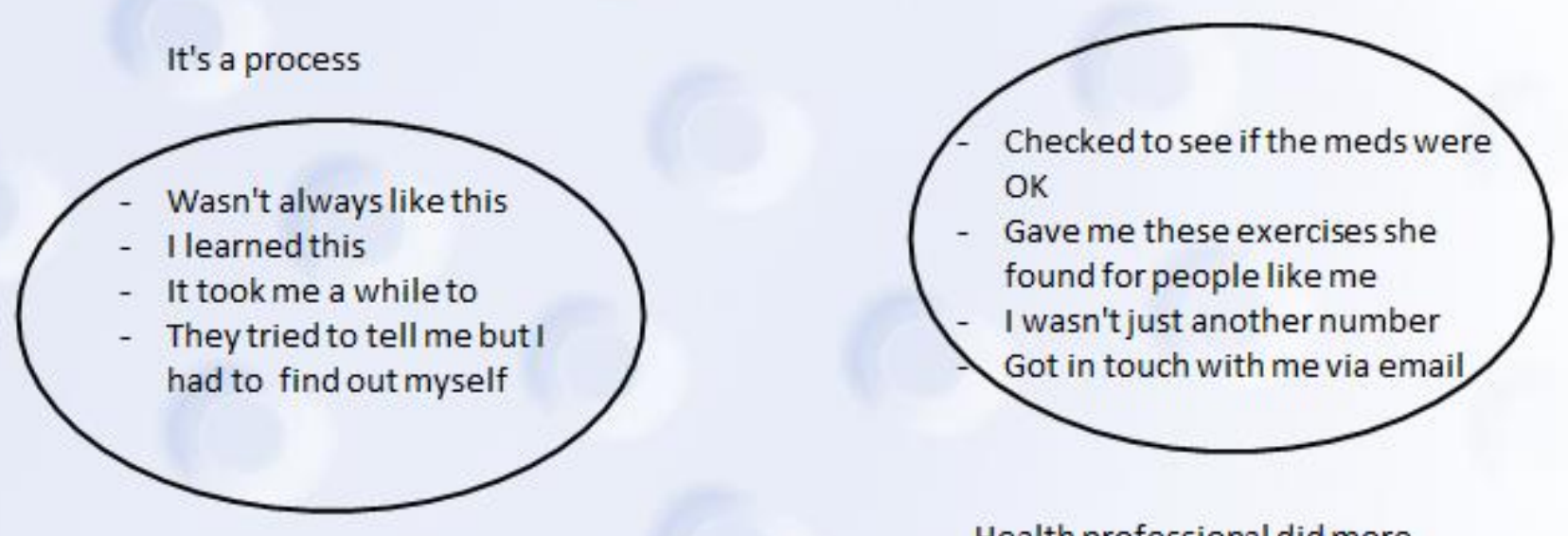

Health professional did more

Figure 6. Excerpt from memo showing four codes (1) attitude to pain; (2) personal attributes; (3) it's a process, and (4) health professional did more 
"I wonder if..." is a phrase echoing throughout my exploration of the data

Curiously, businesswoman doesn't passively accept "this is how it is", she just goes on with what's important in her life irrespective of how she is, while simultaneously searching for more She "knows what's important in life", "uses what's there"

None are tied up in looking for "a cure" but are "getting on with it" no matter what, using "what's there" to do so

Where does "I'm not doing myself any damage" come into it? Is this a cutting/turning point? None of them describe being fearful of damaging themselves

Getting on with it implies - a past, where pain interrupted; a future, where things get done

Figure 7. Memo showing early thoughts about categories

Establishing relationships between categories is an emergent process that only becomes evident as incidents, codes and relationships pattern out (that is, repeatedly occur in the data and explain how the main concern is resolved). I continued collecting data, comparing incidents with incidents to find underlying patterns within the data, and becoming more aware of the various conditions of each concept.

\section{Identifying the main concern and core category (or variable)}

One of the challenges of coding is how to reduce the number of codes appropriately. Glaser identifies that a core category is characterised by being central, relating to many other categories and their properties, accounting for a large part of the variation in a pattern of behaviour, reoccurring frequently, relating meaningfully and easily with other categories, and it carries through to the emerging theory because it is relevant and has strong explanatory power (Glaser, 1978, 1998, 2011; Glaser, 2008; Holton, 2010). Glaser 
points out that "the core category is discovered as it emerges through iterative coding, conceptual memoing, and theoretically sampling for further data to pursue and develop conceptual leads, ensuring that all concepts earn their way into the emerging theory" (Glaser, 2002, para 54).

I was surprised at how quickly the main concern of participants became apparent: each participant used the same phrase "getting on with it" to describe how they were living well. The phrase occurred most frequently in each interview and was the subject of many of my memos. To illustrate, I show two memos, one from relatively early in the project, and one just before moving to theoretical coding in Figures 7 and 8.

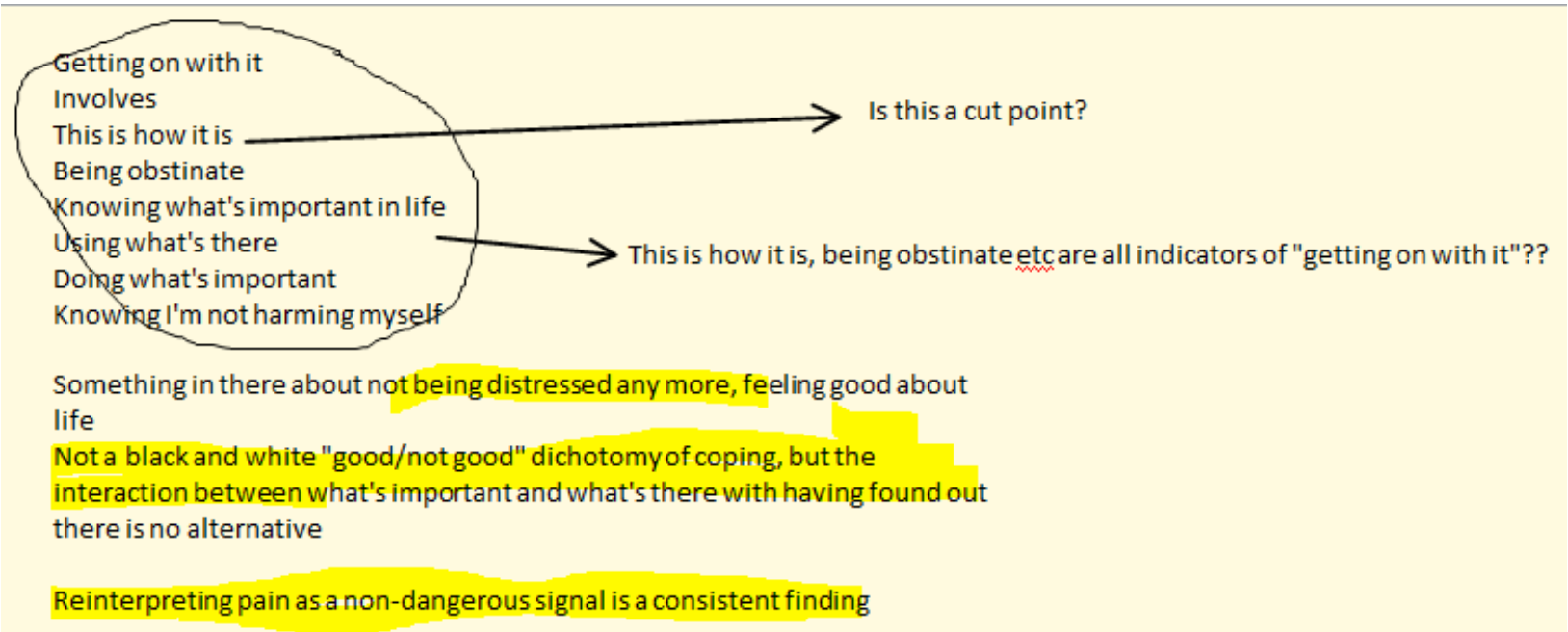

Figure 8. Memo showing coding integration

This memo illustrates the way I integrated codes related to personal attributes (Figure 7) such as "being obstinate", "this is how it is", "knowing what's important in life" to what I later identified as the main concern and core category of getting on with it. Eventually, I integrated the code personal attributes with the category getting on with it, which was a much larger concept that incorporated and explained the many ways participants resolved their main concern. As I reviewed this category towards the conclusion of my analysis, I renamed this category flexible persistence because it more accurately captured the nature 
of the concept. I later named this flexibly persisting. By using a gerund the term is both active (verb) and naming (noun), therefore a gerund captures the nature of an active and ongoing process.

Where does "I'm not doing myself any damage" come into it? Is this a cutting/turning point? None of them describe being fearful of damaging themselves

Getting on with it implies - a past, where pain interrupted; a future, where things get done Getting on with it is associated with putting pain to one side, focusing on what is important, no longer trying to find a cure or pain reduction

Getting on with it recognises that pain doesn't have to be a focus any more, "this is how it is"

- Is it acceptance? They don't use this word much, but when they do it's associated with getting on with it

Getting on with it involves being open to what might be useful in everything they hear about their condition

Getting on with it is what the problem is (main concern) - but it's also how they resolve the problem Getting on with it can begin early on (if parenting or working), but not for everyone, some only get on with it after

- Hearing that this is how it is

- Identifying some important occupation (this is critical to getting on with it - it answers the WHY they get on with it)

- Once they have made sense of their pain, whether thru formal diagnosis or online (Google) or knowing the qualities/what exacerbates/reduces their pain

Some get on with it but are distressed until either they learn hurt doesn't equal harm, OR their it is occupation holds far more reward than hanging out for a cure

Figure 9. Memo recording properties associated with the category "getting on with it"

Figure 9 is an example of a memo written later in the project while clarifying the features of the core category. In it, I identify various characteristics or properties of this category based on incidents provided by the participants. The category personal attributes is now a property of getting on with it, along with other categories such as acceptance (see the note above "Is it acceptance? They don't use this word much, but when they do it's associated with getting on with it", using anything that works, important occupations and mindfulness. 
Classical grounded theory is based on a concept-indicator model. Researchers compare individual incidents with one another such as the many examples of "getting on with it" (illustrated above). Excerpts from three interviews below demonstrate the interchangeability of incidents within the category I had named getting on with it.

"Well, life goes on whether you're part of it or not, so I just decided that no-one else is going to do it, so I needed to get on with life and be_there for my daughter."

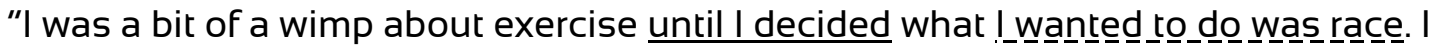
told my parents I would be sore if I did or I didn't do it, and thenen I got into to the gym and started just doing it."

"It's going to be there anyway, and if you wait for your pain to go you'll never do

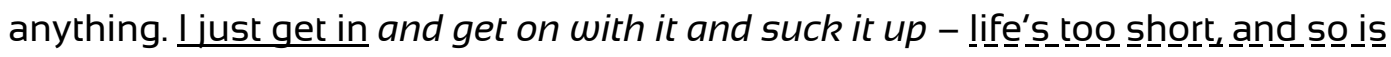
the_e_ugby_s_ea

Phrases in italics represent the category of getting on with it; those underlined with dotted lines illustrate the category of important occupations, and the plain underlining shows examples of this process as a distinct decision in time rather than a subconscious or gradual process.

Even while writing this thesis I thought the core category and main concern were one and the same. They are not. The main concern, although not expressed in these words by participants, is about "getting on with life" but doing so in a way that achieves a sense of self-coherence. Participants often spoke about "needing to be myself", "being who I am", "not letting my pain stop my life", "getting back into my life". I did not discover an appropriate descriptor for this category until I discussed my findings with a Glaserian grounded theorist, when I was challenged to think again about why people might want to get on with life, and what distinguishes people who cope well with pain (and have therefore resolved their main concern) as compared with those who continue looking for 
treatment. The "aha!" moment occurred only after reviewing my memos, recordings and rereading research papers discussing what is so important about being engaged in valued occupation. It was only after connecting ideas about the value of occupation, and why my participants felt satisfied with their lives that I was able to understand that their main concern was to achieve self-coherence. The achieve this by integrating aspects of parts of their old self-concept ${ }^{1}$ and their new self-concept and until they were re-occupying self. The core concept was not "getting on with it" but was about how participants achieved self-coherence, the process of re-occupying the self.

\section{Selective coding and theoretical sampling}

Once I had established that getting on with it (as I had named it) was connected somehow with the main concern and core category, I reviewed my interviews to ensure I had coded all the data related to it. I also reviewed the codes I had previously identified, delimiting and identifying only those codes directly relating to this category. I made the decision to stop collecting data and coding on several codes which had only two or three incidents coded to them, or did not appear to relate directly to the core category: e.g. previous history of pain, activity style, access to health professionals, catastrophising, family with pain, emotional response to pain, occupation, age, gender, literacy.

I then interviewed more participants, this time focusing on how participants made the decision to get on with it, to examine the factors that led to this decision. This is theoretical sampling and involves using emerging categories to explore relevant aspects of the process. I wanted to identify when it occurred, what had influenced the process, and what happened once the person made the decision.

\footnotetext{
1 Self-concept refers to an individual's understanding of how he or she responds in situations and the roles he or she expects to fulfil (Baumeister, 1997; Christiansen, 1999). Self-concept is also a predictive process in which the knowledge an individual has about his or her personality traits, roles, and relationships guide their actions so that he or she presents consistently in both social and private contexts
} 
I found that participants made a deliberate decision to get on with it, but I had not reflected this in the relationship between my codes and the main concern. I returned to my interview data to review the relationship between deciding to get on with it and other codes. I established that although participants often described deciding at a single point, the process involved making sense of pain, accepting that hurt does not equal harm, and weighing up the emotional and energetic cost of pursuing treatment or pain reduction against the rewards of engaging in occupation. I drew a diagram of the relationship between these three categories in one of my many memos from around that time.

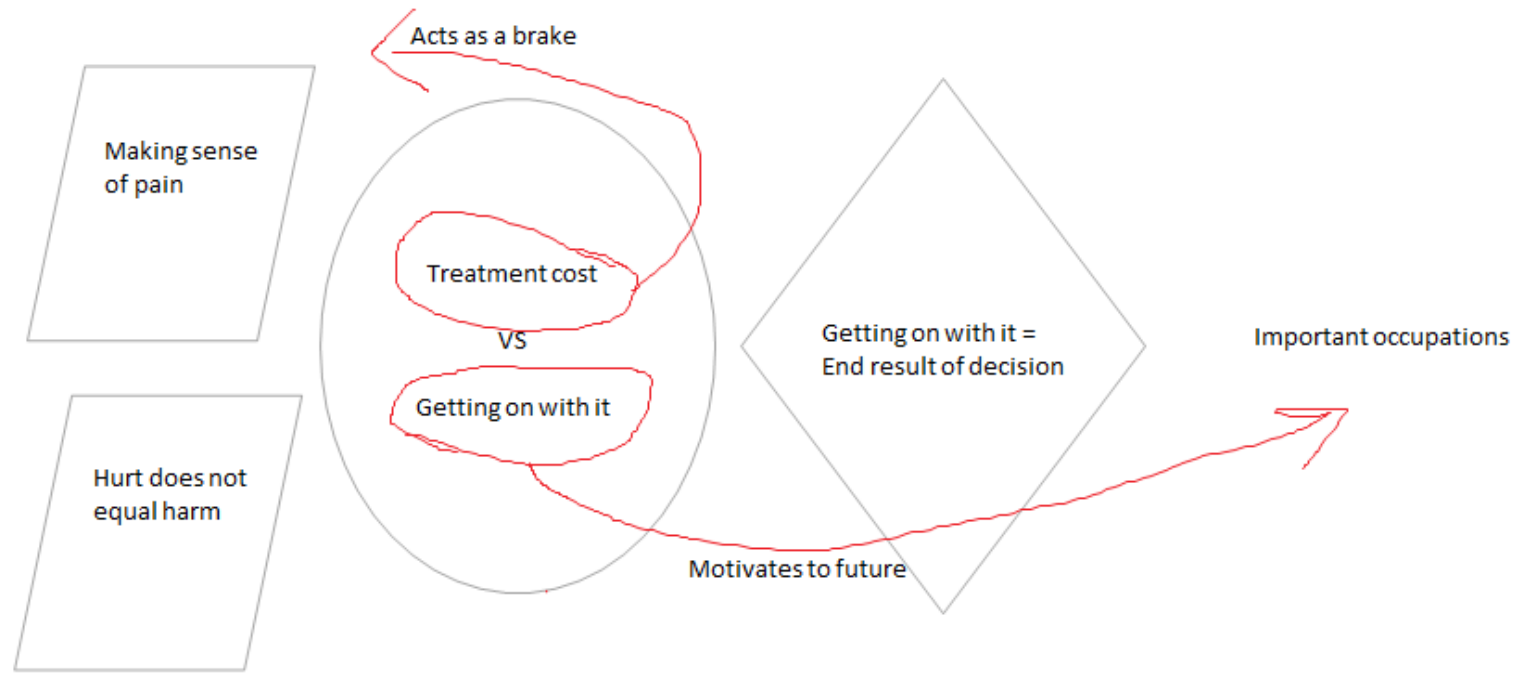

Figure 10. Memo diagram illustrating relationships between three categories

I thought that the category "getting on with it" was influenced by the need to continue with important occupations, but I had the sense that participants experienced conflicting priorities, one of which was the mysterious nature of their health problem. I was struck at the time by some of the participant's descriptions of their process: because information necessary to make a positive decision to get on with life was not always available before they needed to get on with life, some participants described maintaining their roles and responsibilities while still being fearful of hurt and harm, or while their pain was still a mystery. In these instances, getting on with it was accompanied by distress rather than 
the positive approach they described later. Participants described feeling that they could not make plans for the future, they had only enough energy to maintain status quo, fulfilling only those occupational demands they needed to exist, such as remaining at work if they were working, looking for information on their health, trying to understand their symptoms and attending health-related appointments. I needed to understand how people make sense of pain and come to know that hurt does not equal harm. The memo below (Figure 11) illustrates my thinking at the time.

KC says "I had to keep going cos there wasn't anyone else. This was while my pain was really bad, but you have to keep going even though it's killing you because no-one else is there." KC also says "Once I knew what I had it all became much easier even though there wasn't much change in my pain. The worry I was hurting myself ... doing damage - once that was gonel could keep on and not worry about it."

SP says "I didn't like exercise, it hurt and I wasn't fit. I didn't want to do exercise but I had to. It was even more embarrassing to sit out on the side-lines, so I did a bit when I could. Now my pain is the same but l'm working out for a couple of hours every day to keep fit sol can drive. Knowing why I needed to get fit and knowing it wasn't doing my body any harm made a real difference."

CG says "I always kept on doing things but I'd have two groups of friends. Ones who knew me when I was good, and ones who I went to when I wasn't. I didn't do things when I was sore because I thought it was bad for me. Now the only thing that will stop me is if I'm really sick and throwing up. It's like it doesn't matter any more when before l'd be scared and did it only because I knew I had to."

PG says "I only kept going because I didn't want to fail. It was so hard because I hurt so much and didn't know what was going on."

PG also says "Now I make a point of going out for a walk every day. It doesn't always help my pain but it helps me cope,"

\author{
Compare before - pain a mystery, doing = distress \\ After - pain not a mystery/threat, doing = important value
}

Figure 11. Memo illustrating properties of the category "getting on with life". 
As I wrote this memo I realised that making sense of pain, accepting that hurt does not equal harm, and the strength of drive to engage in occupation were factors relevant to a turning point where participants either moved forward so they could get on with it, or remained focused on making sense of pain.

While I could describe, to some degree "what is going on" in the data (Glaser, 1998, p. 91), and I recognised that there was a "before", "after" and a "turning point", I found it difficult to integrate the categories I had found into a coherent model. I had too many categories, and I was not yet ready to relinquish many of them, but they did not work together.

\section{Memos and theoretical coding}

Theoretical coding differs from open and selective coding in that the researcher works to identify relevant theoretical codes by sorting memos and remaining sensitive to the ways they relate to one another rather than working through raw interview data. Glaser (2005) has devoted an entire book to theoretical coding, extending the discussion included in earlier books (Glaser, 1978, 1998). He makes the point that theoretical relationships between variables from any discipline can earn their way into a grounded theory, but warns against actively seeking any particular theoretical code because this can force the theory (Glaser, 2005, p. 30).

I had written over 150 memos before beginning to work on theoretical coding, and generated many more as I continued working. I have provided some examples in the sections above. I would have had difficulty understanding and explaining how I had arrived at decisions about the relationships between incidents and categories, and each of the categories with one another without having documented my decision-making throughout my analysis.

I tried to separate each memo so that it dealt only with one concept when writing my memos, although, in reality, this was not possible. While sorting, when I found memos with 
more than one idea, I duplicated them so I could sort them more easily. I had intended to use NVivo to manage my memos, but I did not find this worked well for me. I ended up cutting and pasting documents from NVivo into Adobe Photoshop, and I began using two monitor screens on my computer so I could look at multiple documents open at the same time. This enabled me to make links between memos as I worked to identify the relationships between the categories.

Most classical grounded theorists endorse hand sorting memos, allowing the researcher to be immersed in the theoretical codes, ideas, connections and similarities between the ideas captured in the memos, and integrating relevant literature into the theory (Glaser, 1998; Holton, 2010; Sandgren, Thulesius, Petersson, \& Fridlund, 2010; Thulesius). I hand sorted electronically, by which I mean that I moved memos around on my computer desktop within Adobe Photoshop. This allowed me to mimic the physical process of cutting and pasting without fear of losing track of documents or taking over every available horizontal space in my home office. I note that other grounded theorists report problems arising from hand sorting physical records because documents can be disturbed during the sorting process (Scott, 2007).

To begin with, having read both of Glaser's books dedicated to theoretical coding (Glaser, 1978, 2005), I first listed all the theoretical coding families that I knew, and then eliminated those that clearly did not fit the relationships I had already found between categories. I reviewed my memos, and initially tried to fit the categories into the six C's Basic Social Process (BSP). This is a BSP found in many different contexts and integrates causes, contexts, contingencies, consequences, covariances, and conditions. A BSP refers to "fundamental patterned processes in the organisation of social behaviours which occur over time and go on irrespective of the conditional variation of place" (Glaser, 1978, p. 100). I tried to organise the categories into the framework of the six C's, by first considering getting on with it as a consequence, with having important things to do (my original code 
for important occupations) forming a condition for individuals to get on with it, and a precondition being the category of knowledge that I'm not harming myself (my original label for the concept of accepting that hurt does not equal harm). Figure 11 shows my thinking at the time. Although I could see there was a process involved in getting on with it, I had also established there was a definite period for most participants when they decided to get on with it. The model in Figure 12 did not wholly capture the quality engendered by getting on with it, which referred to the entire process, and the relationships described by the theoretical codes were not those relationships I had identified from my data. I did, however, recognise that there were two stages, with a turning point positioned between them.

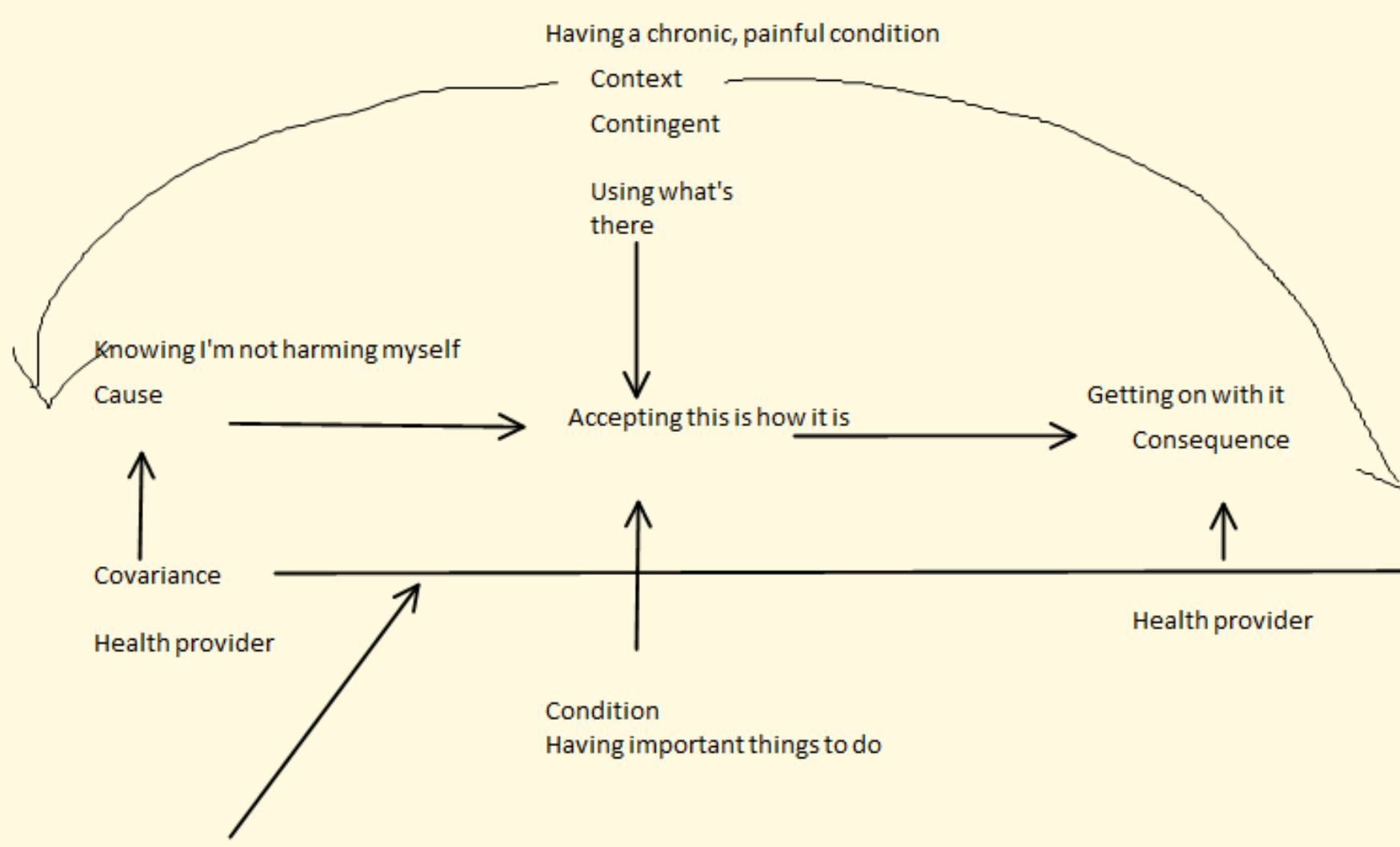

Health provider who does more

Figure 12. Diagram drawn while attempting to fit categories into the "Six C's" basic psychological process. 


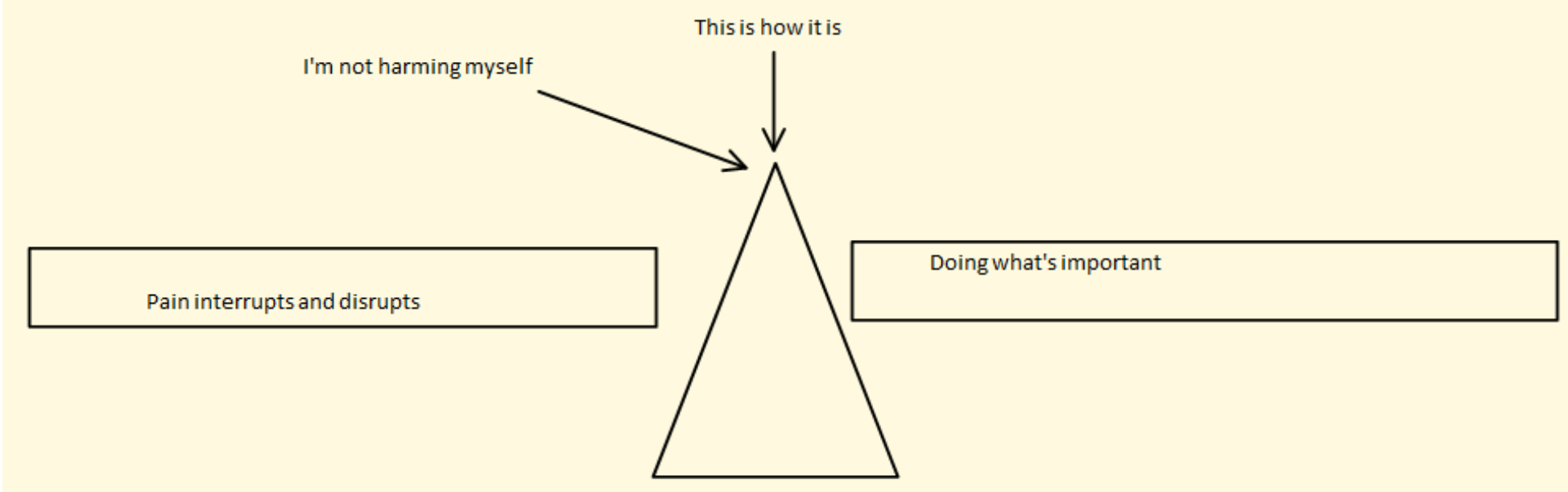

Figure 13. Diagram illustrating "tipping point", then relabelled "Turning Point" and finally called "deciding"

Concepts of "weighing up", along with comments participants had made about "being in limbo land" and the "rush of energy" released that allowed participants to "get into doing what is important to me" led to an image of a car on a journey held back by mud and suddenly released to move forward once reaching solid ground. I reflected in one memo "pain doesn't go away, so it's not like mud", and I doodled images of a see-saw to illustrate the way in which pain stalls forward momentum, the point at which participants weigh up the value of looking for more treatment or alternatively, doing what is important, and the impetus experienced when they decide (see Figure 13).

I wrote the memo illustrated in Figure 14 at that time, thus clarifying the relationships between the categories so far, but I then realised I needed to expand on three categories: important activities "doing what's important"; coping strategies "just getting on with it"; and the positive influence of a "clinician who did more".

I returned to these categories and re-read the incidents I had used as indicators. I will discuss the three categories in turn, but before I do must point out that the way I tried to carry out theoretical coding as described above, is not faithful to classical grounded theory, and I changed my approach after this attempt. Holton (2010) identifies "trying on various theoretical codes for possible fit" can lead to forcing a theoretical structure rather than allowing an emergent fit (p. 36). My understanding at the time I used the strategy 
described above meant I mistook the trial and error approach as being essentially the same as developing theoretical sensitivity from reading and drawing on coding families. In reality, theoretical coding occurs during memo sorting and sorting itself makes the relationships between categories apparent: this is something I learned after my first experiment.

There's a two-part process, divided by a tipping point. Some people get on with it without fully making sense of pain (that's a better label for the first stage - that's what they're doing during that stage), but unless they're happy they're not doing harm, they experience distress if they carry on doing what's important. (know this from comments - see KB - but also from clinical experience with "over-doers" (should I read up on this yet?)

So the first stage is about making sense of pain (because it's a mystery - no catastrophising) and movement into the tipping point occurs once they get a diagnosis (that this is how it is, hurt doesn't equal harm). What happens if they get new pain? Do they cycle back to making sense of pain?

They bring with them, into the tipping point, the personal model of their pain (it's not a mystery any more) and stay there unless they have a clear drive to do something important (hence people not returning to leisure/liferoles after pain management?)

If their drive to do what's important is greater than the stasis in place when pain interrupts, they will move into the second stage.

If a treatment provider doesn't:

- Tell them "this is how it is" (ie no cure, not much more pain reduction)

- Give them a label that fits with their personal model of pain

AND if a treatment provider

- Offers hope of a cure/pain reduction

The person may not get on with it and instead continues with the search to make sense of pain, and pain continues to impede doing what's important or distressing them.

BUT once the person has made sense of pain, knows hurt doesn't equal harm, gets a diagnosis (giving them enormous relief and burst of energy) AND has an important occupation, they begin to get on with it.

Figure 14. Clarifying the relationships between categories, and raising the question of new pain.

To develop theoretical sensitivity involves becoming aware of models from the literature, including models from outside the usual discipline or subject area (Glaser, 1978; Heath, 
2006; Hoare, Mills, \& Francis, 2012). A researcher is more open to seeing relationships between the categories if he or she knows many examples of the way variables can relate to one another. For example, processes are characterised by momentum between categories and often involve stages, time phases or transitions as seen in child development, phases of the moon, and open systems theory. Cut points are likely to involve critical time points, a point of no return, a decision or division, such as in chemical reactions, a decision to go to war, dividing land or property. Strategies may be tactics, mechanisms, techniques or ways of dealing with a situation, person or event.

My knowledge of psychological processes and the biopsychosocial model has influenced the relationships I have identified in this study (for example, self-regulation involving feedback loops, and the negative spirals of pain-related fear and avoidance). This has made my analysis more sensitive to processes, trajectories, and systems. At the same time, the data in my study supports a temporal aspect to living well with chronic pain implying the presence of a process, while the data also provides a clear indication of a point when individuals make the decision to get on with it. The emerging theory thus needed to include both a process over time, and a decision point. I needed to capture these features found in the data as I explored the relationships between the categories.

\section{Developing concepts}

Memos written at the beginning of coding are often quite brief, initially directly relating to data, but as time goes on, and coding becomes more complete, memos document tentative links between categories, capture concepts the researcher may develop, and help the researcher to articulate the reasoning behind decisions. Memo writing helps slow analysis down, giving the researcher time away from being immersed in details within the data and allowing abstract concepts to be more readily recognised; it enables fleeting thoughts during analysis to be documented so they can be used as questions to follow up or to identify comparisons between incidents in the data and extant theory; it documents 
the decision making process as concepts are refined over time; finally it provides the framework for sorting and writing up the theory. The following series of memos shows how doing what's important evolved into occupations and the relevance of this category to the core category of getting on with it and coping strategies.

\section{OCCUPATIONS}

I initially identified "doing what's important" as a category my participants had described. I tried to understand the commonalities between these activities. As I immersed myself in what my participants had said, I was struck by the motivational drive participants had for these activities. I had been considering occupation as defined within occupational therapy and occupational science, and began to read various definitions of occupation to see whether they captured the essence of what my participants told me.

I used different colours to capture the types of activities identified as important by my participants (the examples above are just a selection of those mentioned). I used constant comparison to identify the latent variables represented by the incidents. Initially, I followed an occupational therapy model of occupation in which occupations are categorised as "productive activity or work", "self-care" and "leisure" (Law et al., 1990). I did this because I was familiar with these concepts; however it failed to capture the qualities engendered in the participant's stories and is an excellent example of the way preconceived notions can "force" data into categories without actually representing what data says. I then decided to consider the function or purpose behind these occupations, asking myself "why does this person undertake this occupation?"; "what function does this occupation perform for this person, at this time, in this context?"; along with constant comparison (Glaser, 1998, p.140).

I found there were three main groups, forming three sub-categories within the overall category. I returned to the original recordings to understand the context in which participants described them, and found that they were more than activities, which 
according to Pierce (2001) are shared concepts or general categories of occupational experiences. Instead, they were more accurately termed occupations or personal and meaningful experiences within the context of the individual's life (Pierce, 2001).

Occupations, as used in this sense, are distinct from the lay meaning of occupation in which it is similar to employment or vocation, although employment may be an occupation. The term occupation refers to "the everyday activities that people do as individuals, in families and with communities to occupy time and bring meaning and purpose to life" (WFOT, 2010), and according to Molineux, have five features: active engagement, purpose, meaning, contextual, and human (Molineux, 2009, p. 19).

Participants valued these occupations indicated by the incidents, either because each was intrinsically satisfying or because by doing them the participants could enact values important to them, such as being a good parent or providing for the family. Occupations provided both push and pull motivation (Goossens, 2000) - the 'push' refers to motivation avoiding negative outcomes while the 'pull' refers to motivation towards a positive outcome.

I gave the three sub-categories to pattern out the following names: (1) discretionary, incorporating those occupations an individual deliberately chooses to engage in because of their intrinsic value; (2) supporting health, incorporating the range of occupations individuals do primarily because of their chronic pain condition; and (3) obligations, or those occupations individuals feel they must do to enact important values, often for others.

The diagram overleaf shows how I colour coded these occupations. 


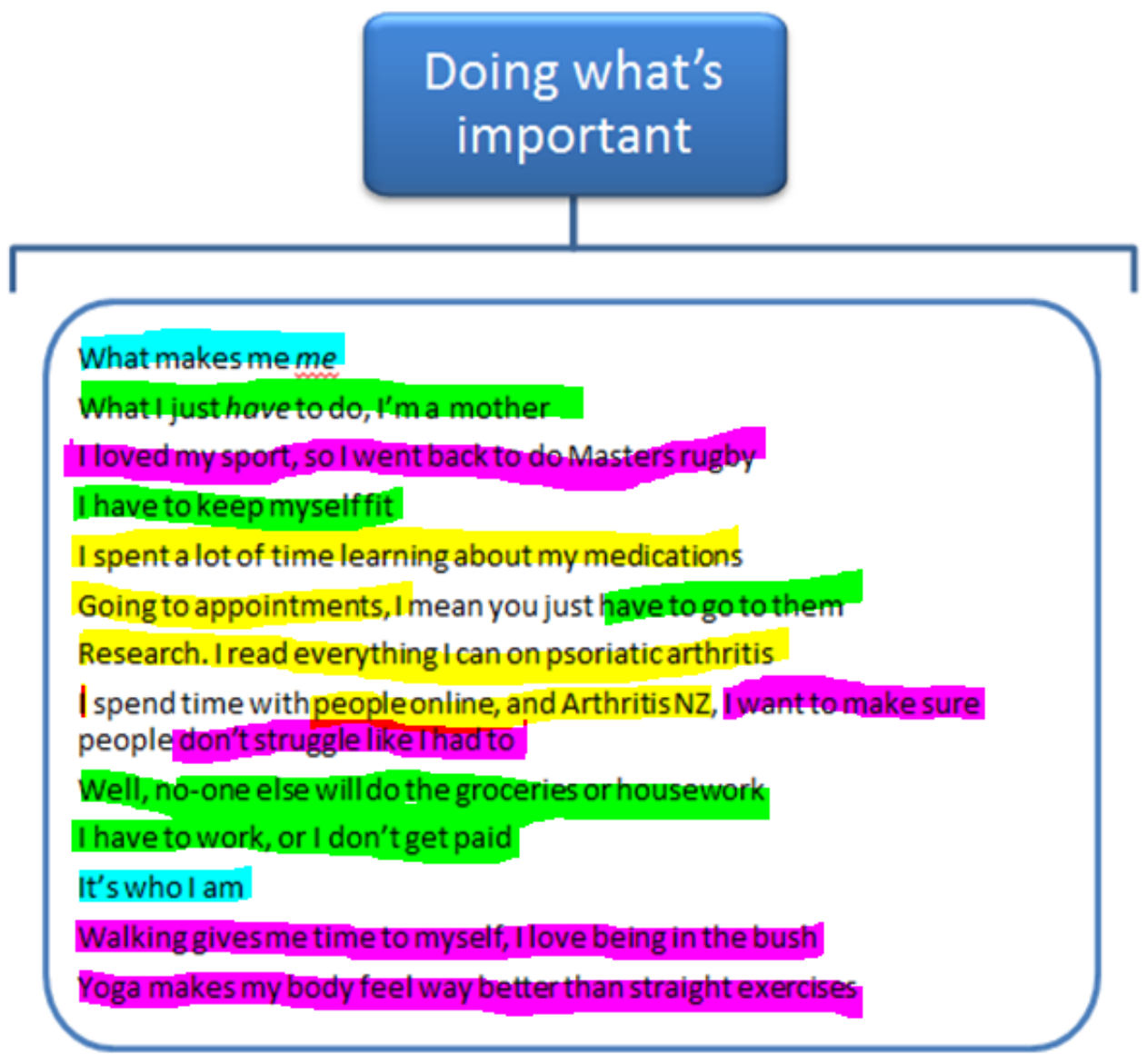

Figure 15. Incidents coded to "doing what's important", later relabelled "Important Occupations"

In the following pages, I provide copies of memos completed while developing the concept important occupations. Memo (a) shows how I considered aspects of "doing what's important", identifying that these activities are personally-relevant, connect with the individual's self-concept, and fulfil obligations. I then consider these as the outcome of having coped, or using coping strategies. Coping strategies and coping enable rather than being an end in themselves. At this point, I posed the question: are important activities, or "doing what's important" equivalent to occupations, as defined in occupational therapy and occupational science. 
Memo (b) is a clip from a table giving a range of definitions of occupation, and I then began writing memo (c). This memo shows my listing the qualities of occupation drawn from my reading of Wilcock (1993), Molineux (2009) and incidents and categories from the data I had collected. It shows how I compared the properties of "doing what's important" with qualities of occupation, and my eventual conclusion that this category represented "important occupations", with coping and coping strategies becoming the means to engage in this while feeling fulfilled.

Using the terminology of Pierce (2001), the category occupations captured the energising and forward momentum of the incidents as described by my participants. Pierce writes:

An occupation has a shape, a pace, a beginning and an ending, a shared or solitary aspect, a cultural meaning to the person, and an infinite number of other perceived contextual qualities. A person interprets his or her occupations before, during, and after they happen...An occupation is the experience of a person, who is the sole author of the occupation's meaning (Pierce, 2001, p. 139).

While I had captured the nature of this category by calling it occupations, I had yet to identify how occupations related to achieving self-coherence or re-occupying self. I needed to look more closely at these relationships, but firstly I wanted to understand more about coping and coping strategies. I discuss this in the next section. 
Figure 16. (Overleaf)Memos considering category "doing what's important" as it evolved into "Important occupations"(a) "Doing what's important" memo (b) Table 2.1 from Molineux (2009) (c) Occupations and doing what's important

Doing what's important - things that need to be done to keep a sense of self? MT "if I don't keep playing I lose something that's part of me" KK "what's the point if you can't do what makes you feel like yourself?"

And things that no-one else can|do (or will do) - CG "I'm the only one who knows how to do the training, so I keep going"

Responsibility-doing what's important

If no-one else is around, these individuals feel an obligation to do it despite the cost. BUT they don't experience it as a cost as much as something that's valuable.

Reviewing a couple of field notes, I was struck by MT's sense that these activities are necessary but also fulfilling, part of identity. They have to get on with it because they have these important things to do which are part of who they are and what they do in the world.

Not all of the activities they do are fulfilling: there's a range of activities they do just to stay well, but they have a reason to do them

SP - go to appts to stay in the good books of my rheumy... gets in the way of things I want to do but it's really important PG - I used to be really fit, now I can't keep up with my old sports so now I make a point of going out for a walk every day ... helps me cope... a bit boring compared with what I used to get up to

KC - you just have to play the game sometimes ... take your medicine and have the blood tests and do the exercises

\section{Parents}

Parents present a special case of important things to do - it's important, but it's not as much about identity, but about obligation and responsibility. Sense of "no-oneelseis around to do it so I must" Parenting necessitates getting on with it because of the sense of responsibility and identity as a parent.

\section{Doing what's important and coping}

Coping is about finding ways of doing what's important. Coping enables doing what's important rather than being separate entity. Coping is always seen in the light of successfully engaging in an activity that's valued. So that's why when ask them how they cope they always refer back to getting on with it, and specific and important/valued things they want or need to do. That last phrase sounds a lot like occupations - is doing what's important the same as occupation? Also coping becomes a facet of getting on with it, as are the important things that need doing. Without one, the other doesn't occur.

\section{Table 2.1 Sample definitions of occupation}

'behaviours whereby humans, collectively and individually, make their place in the physical, temporal and social world (Kielhofner 1992, p. 50)

'the ordinary and familiar things that people do everyday' (American Occupational Therapy Association 1995, p. 1015)

'the activities people engage in throughout their daily lives to fulfil their time and give their life meaning' (American Occupational Therapy Association 1997, p. 864)

'comprises all the ways in which we occupy ourselves individually and as societies' (Townsend 1997, p. 19)

'our occupations are all the active process of looking after ourselves and others, enjoying life, and being socially and economically productive over the lifespan and in various contexts' (Townsend 1997, p. 19)

'specific "chunks" of activity within the ongoing stream of human behaviours which are named in the lexicon of the culture' (Yerxa et al 1989, p. 5)

'an occupation is a specific individual's personally constructed, nonrepeatable experience' (Pierce 2001, p. 139) 
Occupations and doing what's important

Pierce defines occupation as: personal and meaningful experiences within the context of the individual's life

Molineux consider occupation to have five characteristics:

- Active engagement

- Purposeful

- Meaningful

- Contextualised

- Human

See snip (Table 2.1) definitions from other OT people.

So... they're meaningful and have a purpose - fit into life, are valued, require time and energy.

eg making lunch for kids (obliged to, parental responsibility, highly valued)

Playing rugby (chosen, part of identity, pushes self to do it despite pain, highly valued

Racing cars (chosen, part of identity, pushes self to do it despite pain, highly valued)

Walking, gym, exercises (chosen, to maintain wellbeing, important part of routine)

Can I find any activities people have mentioned that are NOT valued for some underlying reason? Any that don't involve active engagement? Or purposeful?

\section{Reviewed values memos and doing what's important - Why does this person undertake this?}

I can't identify any important activities that don't have some sort of value underpinning them. The reasons why people engage in these activities vary but always seem to have a strong sense of why they're doing them.ie they're purposeful and meaningful.

If they're occupations, this implies some of the occupationalscience literature/theory may be relevant?

The choice of words of the American Occupational Therapy Association (1997) question this assumption, as their definition suggests that time needs to be fulfilled, not merely filled. This suggests that it is not just a case of being occupied and busy,

but being engaged in something that contributes to happiness or satisfaction.

Satisfaction-yes! Appears as a quality of the category doing what's important

\section{Wilcock's hierarchy of occupations}

meet survival needs such as obtaining food and shelter

- Induces and enables development of skills necessary for successful living and provides for their maintenance

- Prompts and rewards the use of individualcapabilities to flourish and reach potential

- Expresses social nature

- Means of furthering survival

People are motivated to get on with it because they have occupations they want to/need to engage in, and in order to get on with these things, they use a range of coping strategies. Occupations provide the purpose for getting on with it, while coping strategies provide the means to get on with it. 


\section{COPING}

The next part of developing my theory involved trying to understand how people "just got on with it". At the time, I had started to understand that occupation was an essential feature of the process participants used to "live well", although I had not completely delineated the relationship between occupation and other concepts. "Getting on with it" was the language used by participants, but applied to both the end product (living well), and the strategies participants used to achieve this. I labelled the category "just getting on with it" and incorporated the range of coping strategies participants used when engaging in valued occupations.

Investigating what people do when they get on with it has been the most challenging aspect of this study. While people were able to say that they had got on with it, they found it far harder to elaborate on how they had done so, although why they had was captured in the category eventually called occupation.

I discussed the complexities in understanding coping in Chapter Two. In that section I showed that although there are many ways to classify coping strategies, these discussions assume that coping strategies can be viewed in isolation, can be dichotomised into "good" and "bad", and that people living with pain can remember what they use and when, and that these strategies are used consistently over time (Borkum, 2010; Brown \& Nicassio, 1987; Carroll et al., 2013; Dysvik et al., 2005; Edwards et al., 2009). In this study participants initially had trouble identifying the strategies they used on a daily basis. They often referred to "just getting on with it", so my approach was to ask participants to identify a particular day over the past few days and ask them "take me through your day". Alternatively, I asked how they went about undertaking an important event that had occurred over the past few weeks. This helped to anchor their recall to a particular time or event, ensured the events were recent and enabled me to explore situations in more detail. 
To analyse responses I highlighted each activity using a different colour, and eventually arrived at the three sub-categories below:

1. All participants described using Exercise to cope with their situation. Exercises ranged from traditional forms of exercise such as walking, physiotherapyprescribed exercises, attending the gym or fitness centre, and cycling as well as less traditional forms of exercise including rock and roll dancing, playing rugby, yoga, gardening and geocaching. The defining properties of exercise are intensity, duration, routine and variety and purpose.

2. All participants described using mindfulness or nonjudgemental awareness of pain, although only one person had learned any formal mindfulness techniques. Mindfulness can be conceptualised as a two-component process involving (a) selfregulating attention so that it focuses on what is happening in the present moment, and (b) having an open and nonjudgemental attitude towards what is experienced at that time (Bishop et al., 2004, p. 232). Participants described noticing or being aware of their pain, acknowledging its presence but being emotionally detached or undistressed by it. Instead, they focused on why they were engaging in their important occupations, or on the underlying purpose or values enacted through the occupations. Words used by participants included "noticing", "ignore", "know it's there but don't worry about it", "aware but not bothered";

3. Participants then described a broad collection of strategies that were best captured by the words whatever works. Whatever works was a phrase often used by participants, and referred to their attitude towards being open to strategies they may come across as well as their willingness to vary the strategies they employed at any time, provided that whatever they used enabled them to engage in their preferred occupations. What this meant was that participants did not discount any new approach they learned about, so they were happy to try out various ways of 
managing their pain including using unconventional means (sitting under coloured lights, rubbing creams on the skin, trying out different diets), and conventional means (new medications, new exercises, cognitive therapy, planning and delegating), but at the same time they were not overly invested in reduced pain as the outcome. Their eventual choice of strategy depended on the social context, pain intensity, occupation and future plans, but mostly it depended on their desire to prioritise their valued occupation at that time, even if this meant needing additional recovery time afterwards. Participants described being able to switch quickly from one strategy to another, shifting where they prioritised their energy over the course of a day or week depending on the current priority.

At the time, I thought the three sub-categories were properties of the category getting on with it. Later, these were included in the category coping, forming part of flexibly persisting. Each subcategory, while belonging to coping, also has properties peculiar to that concept. For example, exercise has the properties of intensity, duration, routine and variety and purpose. To illustrate: one participant used a number of different forms of exercise (double lines indicate intensity, dashes indicate duration, solid underlining indicates routine, a dotted line indicates purpose, and a wavy line indicates variety):

"I go for a walk most days. Sometimes l'm too sore so l'll only go for a șhort w wall $\underline{\text { p }}$ and stay close to home or go to my favourite place, a park I can drive to easily after

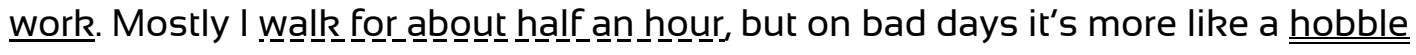

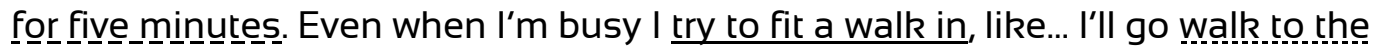
dairy for something... or l'll stroll around the mall. But I do get a bit bored walking, it's not like running used to be. I go on my bike in summer, and when I'm feeling really good I'll bike to work, just for something different. I've even tried aquajogging because no-one can see if you're_not keeping up." 
Mindfulness possesses the properties of noticing, not judging, and timing. Noticing is distinct from being hypervigilant or ignoring, and involves scanning or briefly recognising the location, quality and intensity of pain. I identify noticing in the examples below with solid underlining. Not judging involves being aware of pain but considering it no longer relevant nor eliciting an emotional response, and in the excerpts below it is indicated with dashed underlining. Timing refers to the frequency or duration of using this strategy, and it is indicated by wavy lines.

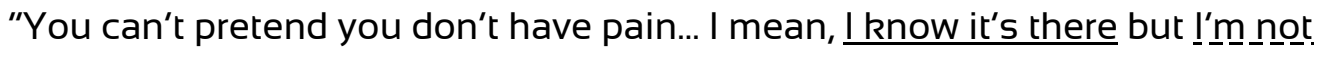
botheresed about it."

"It's always there, but I don't pay it any attention when I'm doing things. It's like, I

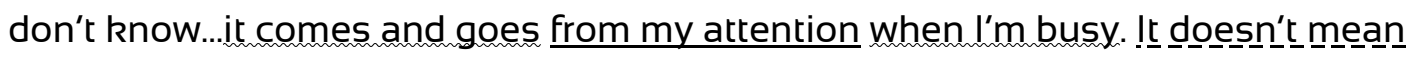

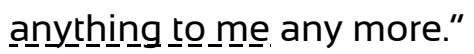

"I do a body scan quite often during the day, you know, go up and down my body in my mind and feel what's going on, but then I go back to my breathing. Pain 는 isn't

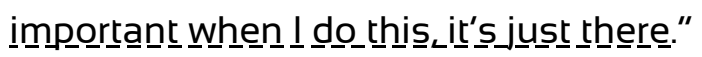

Whatever works possesses the properties of flexibility, open-mindedness, noninvestment, and context. To illustrate the properties of flexibility, I provide examples below:

"If one thing doesn't work, there's always something else you can do" "I see how I feel, sometimes I need to pace, sometimes I decide to ask someone to help, and sometimes I just let things go. It all depends."

"I've learned that you need to be flexible and do things differently just to get on with it."

The following examples illustrate open-mindedness: 
"Well there's more than one way to skin a cat.... might find out something new, something else I can use. Never say never."

"You never say no to learning new things"

"I'll always listen, and then I can decide what I'll take from everything I'm told." Noninvestment refers to a strategy of not anticipating success from engaging in any new approaches to managing pain:

"I'll give anything a go, but I don't want to wait around for anything to work, because you know, it probably won't. It hasn't so far."

"Some people promise you the earth, but I don't hold my breath any more."

Context refers to the range of factors people consider when choosing the strategy or strategies they use at a given time. Context includes balancing priorities, managing energy, time, and physical environment.

"I weigh things up. If I'm too tired I might decide to do less so I have enough oomph for what's really important, being with my kids."

"Well... you can't lie down and relax at work, so I do that at home. What I do at work is the body scan and breathing because no-one knows that's what I'm doing."

"I've raised my desk so I can stand to work. I work flexible hours so I take a bit longer at lunchtime and go somewhere quiet to relax...and I've got good at saying no to people at work.... not quite so good at saying no to family though."

Feedback is an implicit concept when people select coping strategies. Within each coping situation, participants were aware of the desired outcome or outcomes. The outcomes varied but included desired emotional states (for example, happiness, contentment, or joy), meeting an obligation (for example, completing a task, fulfilling a duty), enacting a value (for example, being a dutiful parent, achieving a standard, or being committed to 
community), and experiential avoidance (for example, engaging in an occupation to avoid disappointing another).

Participants monitored discrepancies between their current state and the desired outcome and employed different coping approaches to achieve their intention as conditions vary. Some of this occurs without conscious awareness, and participants found it difficult to describe how they chose strategies, and why they change them over the course of a day. Nevertheless, they were aware that they varied their approach in order to achieve the outcome they wanted. Most of the time the outcome was to remain engaged in their valued occupation despite their pain.

\section{A CLINICIAN WHO DOES MORE}

This third category has posed the most challenges in determining its relationship to the other parts of the model. All participants mentioned developing a collaborative working relationship with a health provider, whether this person was a doctor, physiotherapist, osteopath, nurse or some other provider. The relationship was present at any time since the pain began, but participants did not necessarily continue with this provider. Most participants had developed this relationship around the time or just after deciding to get on with it, but this was not always the case. Indicators for this category are shown in Figure 17. 
Indicators:

Contacted via email in between appointments to check how new medication was going Telephoned with blood test results, whether results were normal or not Looked up something on the internet to follow up on discussion during session Personalised exercises specific to goals

Recorded relaxation from session and gave it (unexpected) Conferred with another provider to check details/treatment Sent brief report direct to person rather than just to GP

Took phone call even though busy If couldn't see on that day, made appointment for later that week Showed resources and how to get them

Arranged a follow-up appointment

Made time

Figure 17. Indicators for category "Clinician who does more", later relabelled "Trustworthy clinician".

Eventually, after considering the outcome achieved from this kind of relationship, it seems to fit most appropriately as a catalyst. Although people do make a decision to get on with life without this type of relationship, the process occurs more quickly when it is present, and the health professional conveys that the patient/client is an individual with unique goals and needs; that his or her health management is a collaborative enterprise; and the health provider will remain available. After the turning point, this relationship supports selfefficacy and encourages function over pain reduction.

Selected memos relevant to this relationship and the thought processes associated with it are below. 


\author{
Health professional musings \\ KC - Dr phones between appointments, looks things up onlinewhile KC is at appointment, conveys \\ sense of personalising the help provided. \\ $\mathrm{S}$ - looks for a Rheumatologist who listens, acknowledges she is a teacher and needs to keep going, \\ hasn't time for infusions etc \\ - see the person as an individual, and does more as a result? Person gives more weight to \\ recommendations because it's aligned to goals/values?
}

\title{
Professional does more
}

KK - physio personalised exercises to fit with time available, looked up info on hypermobility, printed out \& handed to her - KK "meant physio wasn't just giving out the same thing he gave out to everyone... thought how it would fit in my life, said the gym was OK and looked at my programme....asked what I wanted from exercise" - didn't do ex's given by other therapists CG - listened, took time, contacted in between, wasn't satisfied with result - said he'd thought of her when reading a recent article - wasn't making progress until alliance formed with HP S - "I suppose I wanted more from my GP than a prescription. That's why I kept looking around for someone who took more time and came across [osteopath]. He spent longer with me than my doctor, wanted to know what I wanted. Gave me confidence it was OK to keep doing things even though I had pain. I don't see him now because I know what to do but then it was really what made me feel OK to get on with it."

PG - didn't get along with GP. "My doctor is too busy for me. I think she got fed up thinking I was just worrying. I eventually went to see a private rheumatologist, she was amazing. Wanted to know what I wanted and told me we'd work together to get me going again." NB - focused on what she wanted. Sense of collaboration.

\section{Timing}

This relationship occurs at any time - not associated with time of diagnosis, not current in every case, can be intermittent (eg specialist).

Not acted on immediately in terms of getting on with it, but when occupation/making sense of pain/hurt doesn't equal harm are all present, then unless relationship has been made (current or past), person doesn't get on with it.

Acts as a catalyst maybe?

Figure 18. Memo illustrating relationship between category "Trustworthy clinician" and when participants decided to "get on with life". 


\section{Integrating literature}

After I had completed open coding, and delimited and saturated relevant categories, I started to search the major databases (PsychInfo, Medline, Embase, CINAHL, The Joanna Briggs Institute EBP, Google Scholar) using keywords associated with concepts embodied within the category. I began with terms used by participants where possible and linked to the MeSH subject headings. I examined the database thesaurus to ensure the concept had mapped appropriately, and consider alternative terms. I initially used the Boolean operator AND to link the selected heading with the term "chronic pain". I then limited the search to English language and Human participants, and depending on the number of hits, limited the search to the past 15 years. In some instances, I did not link the term to "chronic pain", but used term "pain" or "healthcare", depending on the relevance of literature thus located. I chose to look only at studies written in English soon after beginning to search because of the overall number of articles, and there were few that were not in English.

I was able to identify at least three or four empirical studies for each of the emergent categories, usually more. I then read each article, while making memo notes on OneNote. Using constant comparison, as for any data collected for this study (Glaser, 1998, p.140), I incorporated information from the literature to extend the categories I had identified. I often returned to my interview data to clarify whether categories located in the literature were also in the data provided by my participants. This was particularly relevant when examining the relationship between health providers and people with chronic pain, the coping process overall, and when exploring acceptance and commitment to valued occupations. I had distinct difficulty locating literature on the processes people use to decide to get on with it, and research in people who cope well, with the exception of two studies relevant to understanding coping processes in people who were not seeking treatment (Large \& Strong, 1997; Strong \& Large, 1995). I can consequently claim that the 
data I have collected and the resultant theory contribute unique information about living well with chronic pain.

\section{Theoretical codes}

Theoretical codes explain relationships between the categories that have emerged from open, selected and coding. Many sources can provide theoretical codes, and because these ultimately describe the relationships between categories, need to be reviewed until the resultant model fits and works with the data. In addition to including theoretical codes from the extant literature as well as outside the immediate field of study (Glaser, 1978, 1998, 2005; Holton, 2010), integrative theoretical codes also emerge from the analysis itself, and does so through the process of sorting memos.

The relationships between the categories or variables I have identified have not previously been fully described in other work, although aspects of coping theory such as Lazarus' model (Lazarus \& Folkman, 1984a), Moos and Holahan's model (Moos \& Holahan, 2007) as well as acceptance and psychological flexibility (McCracken, 2010; McCracken \& Velleman, 2010; McCracken \& Vowles, 2007) are certainly relevant. I discuss other theories in Chapters Six and Seven, and point out both similarities and differences between their propositions and those arising from my research.

One model I found particularly helpful was Leventhal's Common Sense Model (Leventhal et al., 1992). While I saw aspects of my findings in the description given by Leventhal and colleagues, particularly the first phase of what I now called making sense, I felt that the Common Sense Model did not provide the reason for wanting to cope. My findings showed that the motivational drive from wanting to participate in important occupations was a critical part of their trajectory towards living well. The memo illustrated in Figure 19 demonstrates my thinking about this model as it relates to my data. I needed to read more. 


\section{Common Sense Model}

Leventhal, Howard, Diefenbach, Michael, \& Leventhal, ElaineA. (1992). Illness cognition: using common sense to understand treatment adherence and affect cognition interactions. Cognitive therapy and research, 16(2), 143-163.

- Self regulation model, developed from treatment adherence, initially focused on conceptualising adherence to treatments

- self-regulation is a function of the representation

of health threats and the targets for ongoing coping (symptom reduction, temporal expectancies for change) set by the representation, the procedures to regulate thesetargets, and the appraisal of coping outcomes.

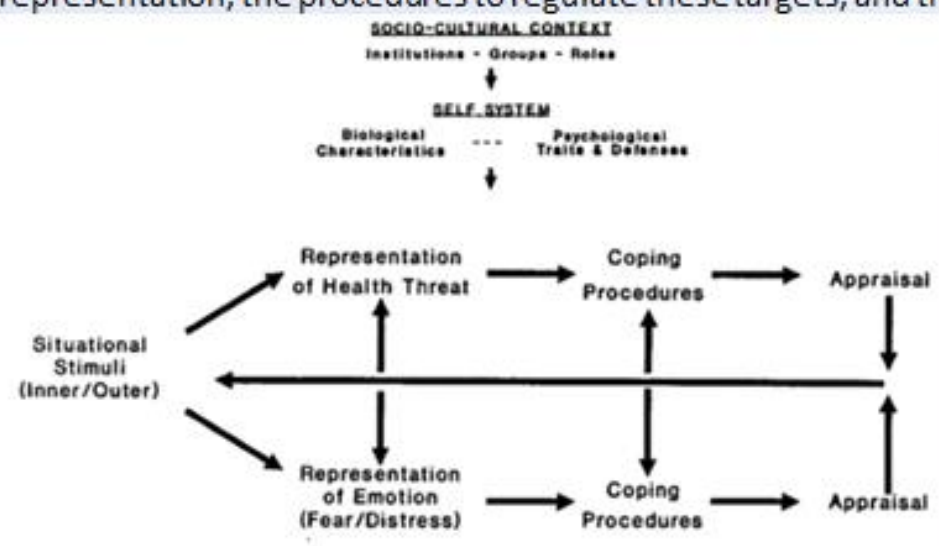

Fig. 1. Self-regulation model. External and internal stimuli generate a multiattribute representation (a subjective perception of a somatic problem) and emotion, leading to coping procedures and appraisals of outcomes. Representations, e.g., targets for self-regulation, coping procedures, and appraisal rules, are affected by self and social context.

Has similarities with categories "making sense of pain", and "deciding to get on with it"

- Situational stimuli are interpreted as a threat (generates need to seek information) and process (whereto go for information, who to see, who is authoritative)

- Was developed after observing that both the emotion-producing stimulus as well as the action stimulus were required for behaviour to occur

- Identified that both representation of the health problem AND the action plan determined coping responses

In reference to my findings, making sense of pain involves identifying that pain experienced is not the same as "normal pain", therefore it's mysterious (similar to illness representation representation). People interpret what's mysterious for them on the basis of experience, external sources and communal beliefs about illness.

Once identified as mysterious, people begin looking for verification/labeling: a process of seeking diagnoses that match with their personal experience AND expectations.

Diagnosis-seeking includes seeing health professionals who should, in order to facilitate coping, provide verification/labels concordant with the individual's beliefs/experience-cause, consequences, control/cure, identity, timeline-If the diagnosis/label is concordant with the individual's experience, diagnosis = relief, which equates to reduced emotionalillness representation.

Therefore: CSM supports the first phase of my model, in particular the need to identify a diagnosis that is aligned with their experience.

What's missing is WHY someone might want to cope - and the context of coping, although they incorporate the sociocultural context in the original model, while later discussion of the CSM does not include context.

Figure 19. Memo discussing Leventhal's model and my findings 


\section{Integrating}

It was while reviewing various references that I began to consider why engaging in occupation was such a motivating force. I reviewed some of the occupational therapy and occupational science references and discovered that some theorists believe that occupation is an expression and creator of self-identity (Christiansen, 2000; Christiansen, 1999). I was concurrently reading about "possible selves" as this idea was captured in research examining sense of time and future-orientation (Markus \& Nurius, 1986; Morley et al., 2005; Sutherland \& Morley, 2008). I also reflected on how experiencing chronic pain represented a loss of familiarity: familiar body, familiar healing process, familiar treatments and even familiar relationships with family, treatment providers and self. It was at this point I began to consider that the main concern of all participants, including those who did not currently believe they were "living well", was achieving self-coherence. How could people develop an understanding of their new world? One that made sense, along with a body that made sense? I recognised that occupation provided the means for people to move from being a patient to expressing their self-identity, and that those who were most successful in achieving this had integrated pain as part of their self-concept. By integrating aspects of "self-with-pain" into an overall self-concept, individuals had resolved their main concern so that the world and their identity made sense again (was coherent). The process of achieving this was through re-occupying self, as in "re-inhabiting self", or feeling at home in the new self-concept. I had my main concern, and the process of resolving it. By working with the remaining categories, I was able to explain how and why some people learned to "live well", and quite unexpectedly, also explained how and why some people did not. I now had a grounded theory of living well with chronic pain.

In Chapter Six I situate this theory within the existing body of knowledge as I demonstrate how I have woven concepts from extant theory into the findings from my study. 


\section{Theory structure}

A relatively straightforward theoretical model for the process of living well with chronic pain emerged from the data I had collected. The main concern of people who cope well with pain is achieving self-coherence after the onset of chronic or persistent pain. This concern is resolved by the process of re-occupying. Re-occupying occurs in two stages, the first involves making sense, and the second is flexibly persisting. Re-occupying means achieving a coherent self-concept in which important values are enacted by participating in important occupations, roles and the future self.

In the process of making sense, individuals seek diagnostic clarity, develop symptom understanding and engage in occupational existing. Diagnostic clarity gives individuals a label which provides social validation and helps indicate the long-term prognosis. Importantly, diagnostic clarity also informs individuals that their pain does not indicate a serious threat; that hurting does not mean bodily harm. Symptom understanding involves individuals developing a personalised model of their symptoms and the variations they can expect. Occupational existing means maintaining only those occupations that are necessary, while simultaneously learning about the relationships between pain and activity.

Individuals reach a turning point when they begin deciding before progressing towards flexibly persisting. Deciding is the time when individuals weigh the cost of focusing on their health alone against the benefits of engaging in valued occupations. For people who achieve self-coherence and re-occupy self, this is a positive step towards flexibly persisting. For some, however, the strength of occupational drive can prematurely turn them away from making sense, and in these circumstances although they may participate in occupations, they also feel distressed and perhaps fearful of the consequences. $A$ 
trustworthy clinician may be influential at this time by providing support and encouragement to pursue occupations, acting as a catalyst to deciding. Clinicians, who focus on investigating symptoms, or obtaining more treatments aimed at reducing pain or finding a possible cure, may delay or even prevent a person from deciding.

Individuals begin flexibly persisting after deciding. This process is an ongoing, fluid allocation of resources to ensure the person remains able to engage in valued occupations. Occupational engaging involves the person participating in occupations that fulfil important values and enable re-occupying. Coping involves using the resources to hand to achieve what is important. Coping may target pain reduction or task completion depending on the occupational focus at the time. Future planning begins again, having stopped while making sense. Future planning means having a focus beyond immediate symptom or health management, towards valued outcomes.

\section{Summary}

In this chapter, I have demonstrated how I have followed classical grounded theory methodology strictly, from my initial need to reformulate my research questions through to completing the analysis and writing up. I have established my reasons for choosing classical grounded theory over other versions of this methodology, and documented the process of collecting quality data. I have traced the process of analysing my data as I became more familiar with the methodology and included memos to demonstrate how I arrived at crucial analytic decisions. Finally, I have briefly summarised the grounded theory that emerged from my data. In the next chapter, I provide a comprehensive description and discussion of a grounded theory of living well with chronic pain. 


\section{Chapter Five - A model of living well with}

\section{chronic pain}

This chapter presents a classical grounded theory (model) of living well with chronic pain. The model has emerged from constant comparison data analysis of interviews with individuals with chronic pain. The chapter details the main contribution to knowledge drawn from the empirical study on which this thesis is based. Data collected over two years forms the primary basis for this model, rigorously obtained and analysed following the tenets of the classical grounded theory methodology. These findings will be compared and contrasted with relevant extant literature in Chapter Six when I situate and discuss the theory.

I begin this chapter by outlining the grounded theory and its important characteristics. I will then show how the process of re-occupying self provides a theoretical explanation for how people, who live well with chronic pain, resolve their main concern of achieving selfcoherence. I then detail the main contributions to knowledge given by this grounded theory.

\footnotetext{
A note about terminology: Words or phrases in italics indicate categories, codes or properties. Categories and codes are labels given to latent variables identified in the data and are equivalent to factors or variables in other methodologies. Subcategories may be identified within a category and can be properties or dimensions. Properties are characteristics of a category such as variability, rhythm, measurement while dimensions refer to scale or size.
} 


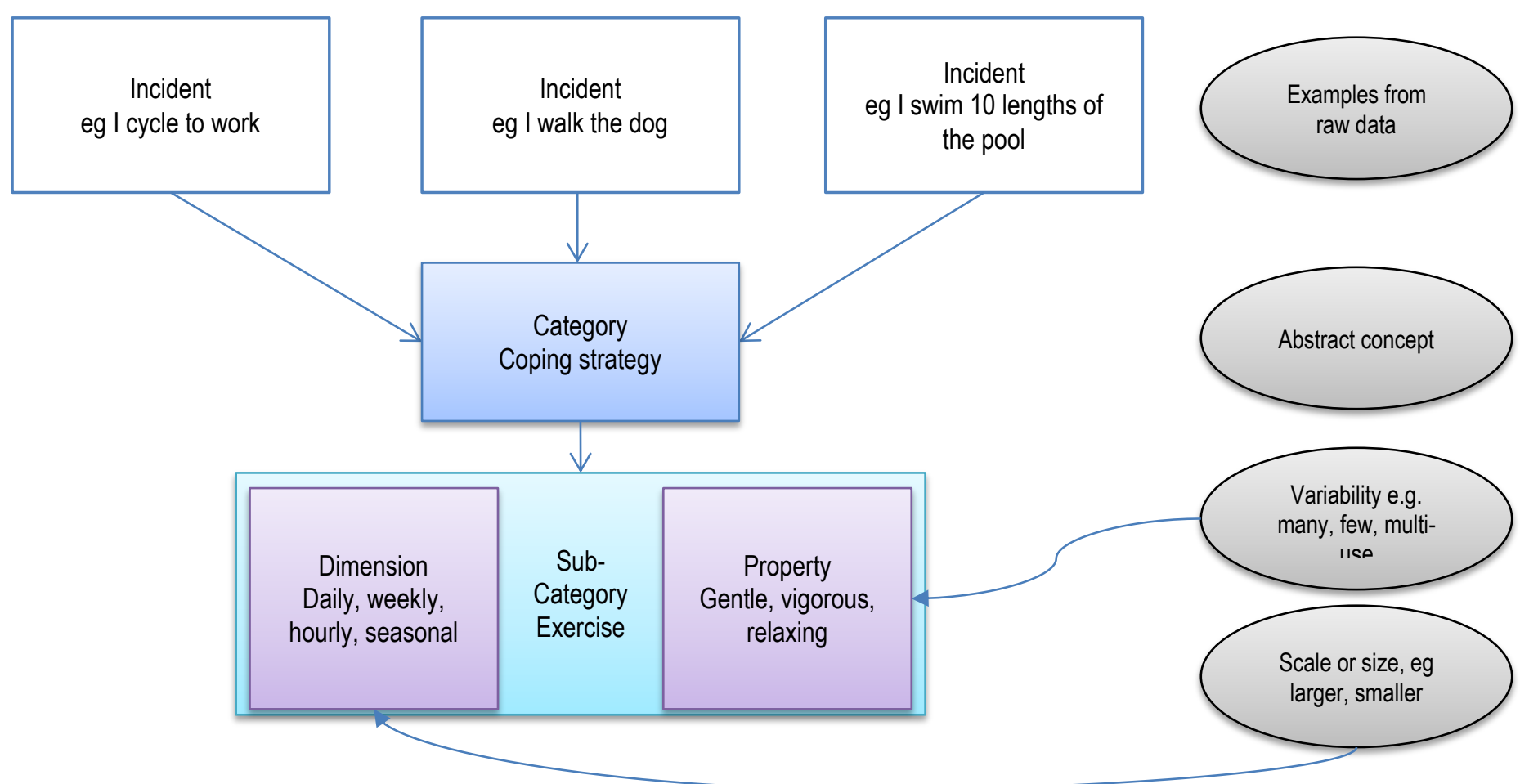

Figure 20. Illustration of relationships between data and conceptual analysis

\section{A mid-level substantive theory}

This is a mid-level substantive multivariate theory. It explains the two-phase process individuals follow as they move from pain onset, through to a relatively stable phase when they describe themselves as "living well". The theory contributes to a greater understanding of the ways people cope with chronic pain: in contrast to most research into chronic pain; it focuses on those who cope effectively. The processes involved in the trajectory towards living well with chronic pain involve reorganising the self-concept, so aspects of self that can no longer be achieved are relinquished, and new practices and values are adopted so that the individual can believe the world is an understandable place, and they occupy or live comfortably in the new self-concept.

In addition, the findings identify key factors that have influenced individuals' positive trajectory. The motivating power of occupation is a defining feature of the model, explaining why people develop ways of coping and how they use them. Occupations are 
"the everyday activities that people do as individuals, in families and with communities to occupy time and bring meaning and purpose to life. Occupations include things people need to, want to and are expected to do" (WFOT, 2010). Occupations are the unique and individualised way in which a person enacts a particular activity (Pierce, 2001), and are characterised by (1) active engagement; (2) being purposeful; (3) meaningful; (4) contextualised and (5) human (Molineux, 2009, p. 17).

Coping is reconceptualised from a taxonomy where strategies are often dichotomised, such as passive or active (Folkman \& Moskowitz, 2004), towards a functional and contextual interpretation (Paez-Blarrina et al., 2008; Van Damme et al., 2008). Coping strategies are viewed as more or less workable, depending on how well they support the individual in the context of his or her life.

\section{The main concern and its resolution}

\section{Achieving self-coherence}

The main concern of people experiencing chronic pain is finding ways of achieving selfcoherence. Chronic pain presents a threat to identity because it does not go away as individuals expect based on their past experiences with acute pain, and because it provokes negative emotions and interferes with normal function. The onset of pain disrupts the usual forward trajectory of life, causing a temporary stasis and generating uncertainty about plans for the future (Hellstrom, 2001). Pain interferes with the future or ideal self-concept by imposing a current self-concept complete with restrictions and uncertainty upon the individual's sense of self. Pain presents uncertainty for the future; uncertainty about life, work and relationships. The world that was, is disrupted. Individuals experience a reduced sense of coherence in the face of chronic pain. 
Sense of coherence is a concept of the world as generally comprehensible, manageable and meaningful, so that in the face of a stressor, people are motivated to cope (the world has meaning), believe that the challenge can be understood (comprehensible), and that they have the resources to do so (manageability) (Antonovsky, 1996, p. 15). Sense of coherence is thought to be a salutogenic orientation, or a view of the world that helps people remain healthy or resilient in the face of stress (Antonovsky, 1996; Eriksson \& Lindström, 2005, p. 460).

When circumstances change, when individuals cannot find meaning in what is happening or has happened, and/or when their usual coping resources no longer work, sense of coherence is reduced. Participants in this study identify this state as "being in limbo land". In particular, their sense of self-identity is challenged, along with the negative emotional impact of their pain. Self-identity is "the person we think we are", or "the self we know" (Christiansen, 1999, p. 577), and is linked to how well individuals know their own abilities and limitations; how well they accommodate limitations and enhance abilities when fulfilling social roles; how comfortable they are with their capacity to meet others' expectations, and how they construe the future. Chronic pain can constrain all these aspects of self.

\section{Re-occupying self}

To achieve self-coherence, or the sense that the world and self are understandable, manageable and meaningful, people living with pain begin the process of re-occupying self. Re-occupying self means making sense of the situation, deciding to get on with life again, and flexibly persisting in important occupations despite the ongoing effect of pain on function. Ultimately, re-occupying self means achieving self-coherence by reconstructing or developing a self-concept in which the things that give life value and meaning can be achieved and experienced, although perhaps in different ways. 
Self-identity incorporates self-schemas ${ }^{2}$ or specific concepts of various features of the self (Baumeister, 1997, p. 615). Chronic pain creates a discrepancy between what an individual expects to be able to do (aspects of the self-schema such as competence), and what he or she can do. The negative emotions arising from this discrepancy motivate action and, to the extent that functional limitations from pain interfere with being able to engage in valued occupations, they threaten those aspects of self-identity that are based on competence. Thus, the first phase of re-occupying self with chronic pain involves making sense; to acknowledge the discrepancy between the current and ideal self through occupational existing, or encountering what occurs while doing the occupations of daily life; developing symptom understanding, or an awareness of the meaning and fluctuations in symptoms; and to obtain diagnostic clarity, to clarify future expectations about the disease process and its management.

The second phase of re-occupying self with chronic pain involves flexibly persisting so that occupations are maintained, embodying valued aspects of self-identity. Flexibly persisting invokes the moment-by-moment flexible allocating of time, energy and resource used to remain engaged in valued occupations despite ongoing pain. Highest priority is given to occupational engaging, or doing the things that make for a good quality of life. Occupations differ from person to person and shift over the course of a lifetime, but underneath the superficial details lie essential values, habits, and aspects of self-concept that define self-identity. Flexibly persisting involves using coping. Coping refers to the range of strategies people employ to accommodate the limitations imposed by chronic pain and to engage in what is important to them. Coping may include decisions about the way the day is structured, the amount of energy allocated to a particular occupation, using

\footnotetext{
${ }^{2}$ Schema are "cognitive structures of organised prior knowledge, abstracted from experience with specific instances; schemas guide the processing of new information and the retrieval of stored information" (Fiske \& Linville, 1980, p. 543).
} 
an adaptive tool or process, or ways to increase emotional or mental capacity. Finally, flexibly persisting initiates future planning that has been on hold during the first phase of re-occupying self. Future planning is able to occur after making sense because individuals have a greater sense of self-coherence. Life becomes more predictable after making sense, enabling the person to begin the process of flexibly persisting. Flexibly persisting allows dreams and plans for the future to become a reality.

Flexibly persisting allows individuals to incorporate necessary changes into self-schema (ways of doing things, values, expectations and so on) so that self-identity is unified and feels comfortable. The actions may include adopting coping strategies, temporarily modifying the self-schema by making allowances for the current state, or redefining the self-identity to reduce this discrepancy. This process reduces negative emotions associated with the self-concept discrepancy and enables individuals to renegotiate and re-occupy self.

Between the two processes of making sense and flexibly persisting lies deciding, a turning point. Deciding occurs after making sense, when the self-concept is still fragile. It is then, while re-occupying self is incomplete that individuals begin to let go of aspects of the selfconcept that cannot be retained. Two main factors influence deciding: clinicians and occupational drive. Clinicians may influence individuals to either continue making sense, perhaps by suggesting more investigations or offering treatments that could promise a return to "normal" (thus removing the need to re-occupy self or achieve self-coherence). Alternatively, clinicians may reiterate the need to let go of the "old normal" and actively support occupational engaging, coping and future planning. Contributing to deciding is an individual's own occupational drive. Occupational drive generates forward momentum, propelling individuals to find ways of flexibly persisting. 
There are two essential features of the process of re-occupying self: the influence and lived experience of time and temporality, and movement within and between the processes is at times mutable and stable.

\section{Main concern $=$ achieving self-coherence}
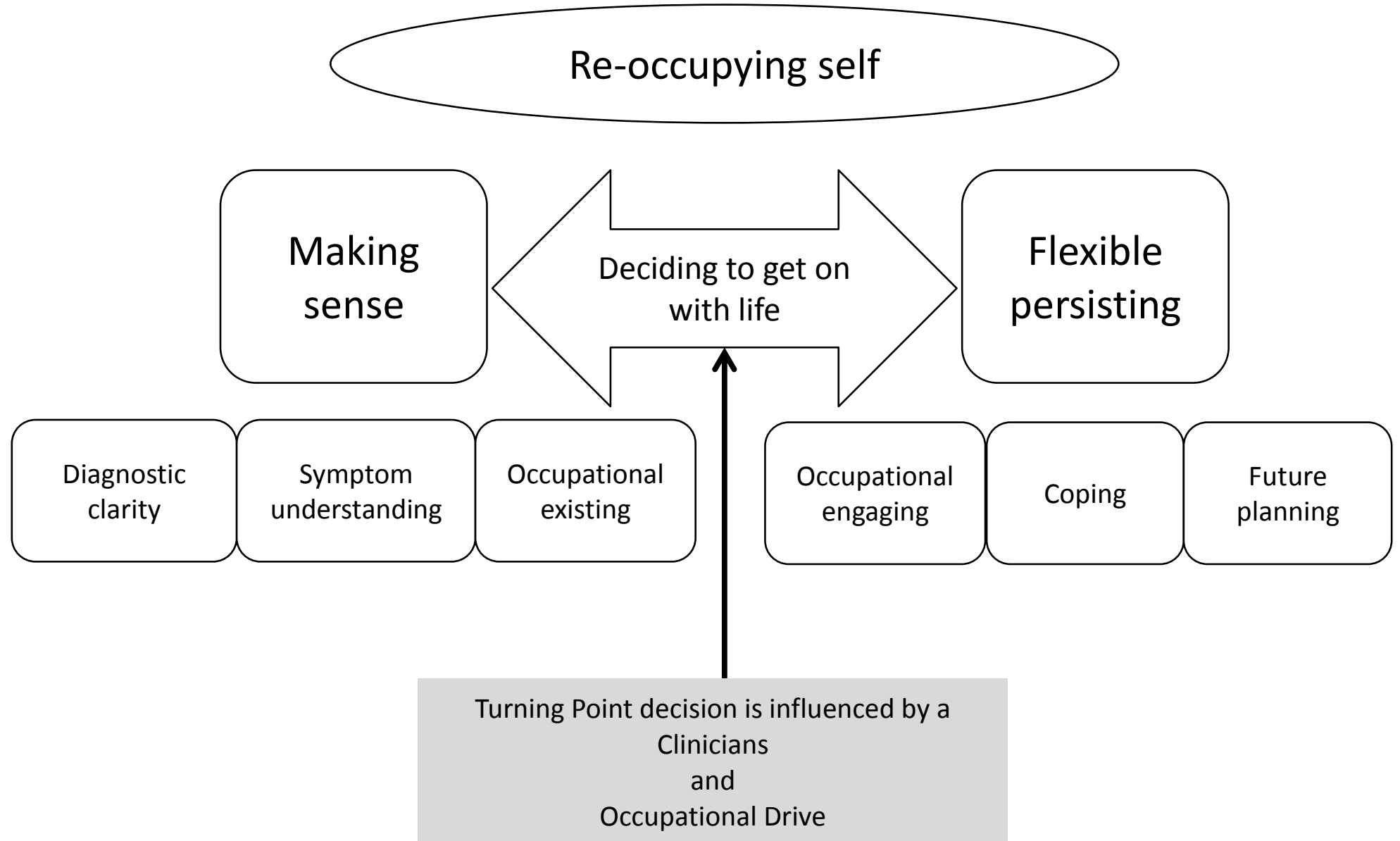

Figure 21. Visual representation: Achieving self-coherence by re-occupying self 


\section{Characteristics of the model}

\section{Influence of time}

There is a temporal dimension to the process of re-occupying self. Life prior to the onset of pain is a reasonably predictable trajectory that is aligned with an individual's sense of identity (roles, responsibilities, habits, plans, expectations, values). Pain disrupts this trajectory, and some of the individual's energy diverts to managing the problem of pain. Planning for the future slows or stops entirely, and the period may be described as "limbo land". Planning reduces because the sense of coherence, both of the world and selfconcept, is reduced, and the future no longer feels secure.

Distress relates at this time to the degree to which pain (and other symptoms) disrupts the possible, future or ideal self (Morley et al., 2005). Limbo land is what Hellstrom and Carlsson describe as "a viscous long-lasting now" (1996), in which the future seems murky, and the present stands still. It can be an emotionally demanding period because the outcome of future plans is now uncertain. The more time needed making sense; the longer life is in limbo while plans are placed on hold. Although many individuals remain involved in important occupations during this period, they must also allocate energy to the making sense process. The cost of allocating resources to occupational existing while making sense means less energy is available in reserve for activities or events that are out of the ordinary such as Christmas, travel or family tragedy. Occupational choices become habitual and routinised so that individuals need less thought and planning to maintain roles and responsibilities. Plans for the future are particularly affected and become less well-defined because the person's self-concept (prior to pain onset) is no longer completely valid, and the future is uncertain. 
To return to forward momentum involves two phases: making sense when pain disrupts the life trajectory and the present and ongoing process of flexibly persisting. Deciding to get on with life divides the two phases and marks a point when the forward trajectory begins to resume.

During the process of making sense individuals may spend a great deal of time and effort trying to match their experience with information both from within the community (for example family, friends, and support groups) and that provided by health professionals (for example medical practitioners, physiotherapists, chiropractors). This period can last years but may also be as short as several months. It terminates when the individual has made sense of his or her pain and weighs the cost of pursuing treatment against the rewards of engaging in valued occupations. Deciding to engage once again in valued occupations begins the forward trajectory of planning for the future. Once an individual makes this decision, even when new symptoms arise or pain fluctuates, individuals continue to focus on engaging with valued occupations, and rarely if ever, return to the static and disrupted phase of the onset of their problem. Flexibly persisting is identified by a return to focusing on the future, when life is not dominated by symptom management, and when plans can be made again.

\section{Mutability and stability}

The paths through the two phases of learning to live well with chronic pain are fluid and of no set duration. Instead, the phases are defined by characteristics such as future orientation, sense of coherence, and accepting that the self-concept has changed but still represents "who I am". At the same time, the generally stable phase of flexibly persisting remains dynamic because pain intensity and quality varies, the context in which people live influences their actions and priorities each day while new information about ways of coping emerges from time to time. This generates an on-going dynamic between 
managing pain and living life, with individuals continually redistributing energy across occupational domains. During my study, this was particularly relevant for the participants living in Christchurch, given the impact of the earthquakes between September 2010 and December 2012. Nonetheless, individuals reaching the second phase demonstrated a sense of identity, purpose and future-orientation once they decided to get on with life, contrasting sharply with the earlier period of making sense.

As Cassell (1999) says, individuals at this time no longer experience the "... specific state of distress that occurs when the intactness or integrity of the person is threatened or disrupted ... [that] ... lasts until the threat is gone or integrity restored" (p. 531). Importantly, entering the second phase of flexibly persisting does not indicate a return to normal. "The new normal" is a somewhat clichéd expression, but over time, individuals who cope well create a new self-identity that carries some of the past self through into the current self and future plans. It is only by successfully achieving the tasks involved in making sense and flexibly persisting that a positive new normal ensues.

NB: I have used the term "phase" as opposed to "stage" within this model because stages usually describe qualitatively distinct and sequentially ordered periods such as developmental stages while phases involve transitions that may overlap or be completed at different time points such as changing fashions.

I now discuss each of the phases and the sub-processes involved in achieving selfcoherence by re-occupying self. At this stage, it is important to note that individual participants in this study did not use the words "self-coherence" or "re-occupying self". These terms emerged as concepts derived from the examples provided by participants that have patterned out to indicate the underlying construct. Nevertheless people, who identify as "living well" with chronic pain, have strongly identified with the process as I 
describe it, supporting my contention that this theory fits, works and has relevance in the substantive area.

\section{The processes of making sense}

When the doctor explains to the patient what's wrong, he imagines that she is a blank slate - baffled by her pain and waiting for an explanation that she will wholeheartedly accept, since he, not she, has the correct information. If she had any interpretation of her pain before, she will naturally immediately relinquish it in the face of his overwhelming expertise. (Thernstrom, 2010, p. 217), p. 217.

The first phase of re-occupying self primarily involves making sense. While individuals are making sense they allocate personal resources to the process, and consequently need to modify temporarily, or even relinquish some occupations while continuing to do their most valued ones. They engage in three primary processes while making sense. On completion, these processes ensure the person is ready to consider moving forward again.

Until their pain became a problem, people who live well experience the usual ebb and flow of life. They have certain occupations they enjoy, envisage a future in which they have entertained "possible selves", or "cognitive representations of enduring goals, motives, aspirations, fears, and threats" (Hellstrom, 2001; Markus \& Nurius, 1986). Their pain may come on insidiously, and individuals may initially dismiss their pain as temporary and of little consequence. When it persists, changes in character in some way (increases intensity, changes quality, becomes associated with other symptoms), and no longer fits with a previously experienced pattern of musculoskeletal pain, people start to investigate their problem, and see the need for making sense.

There are many ways individuals begin making sense; one may be to see a healthcare provider, but individuals may also talk with family, friends and colleagues, conduct an online search ("Dr. Google"), contact organisations such as Arthritis NZ or read popular magazines. 


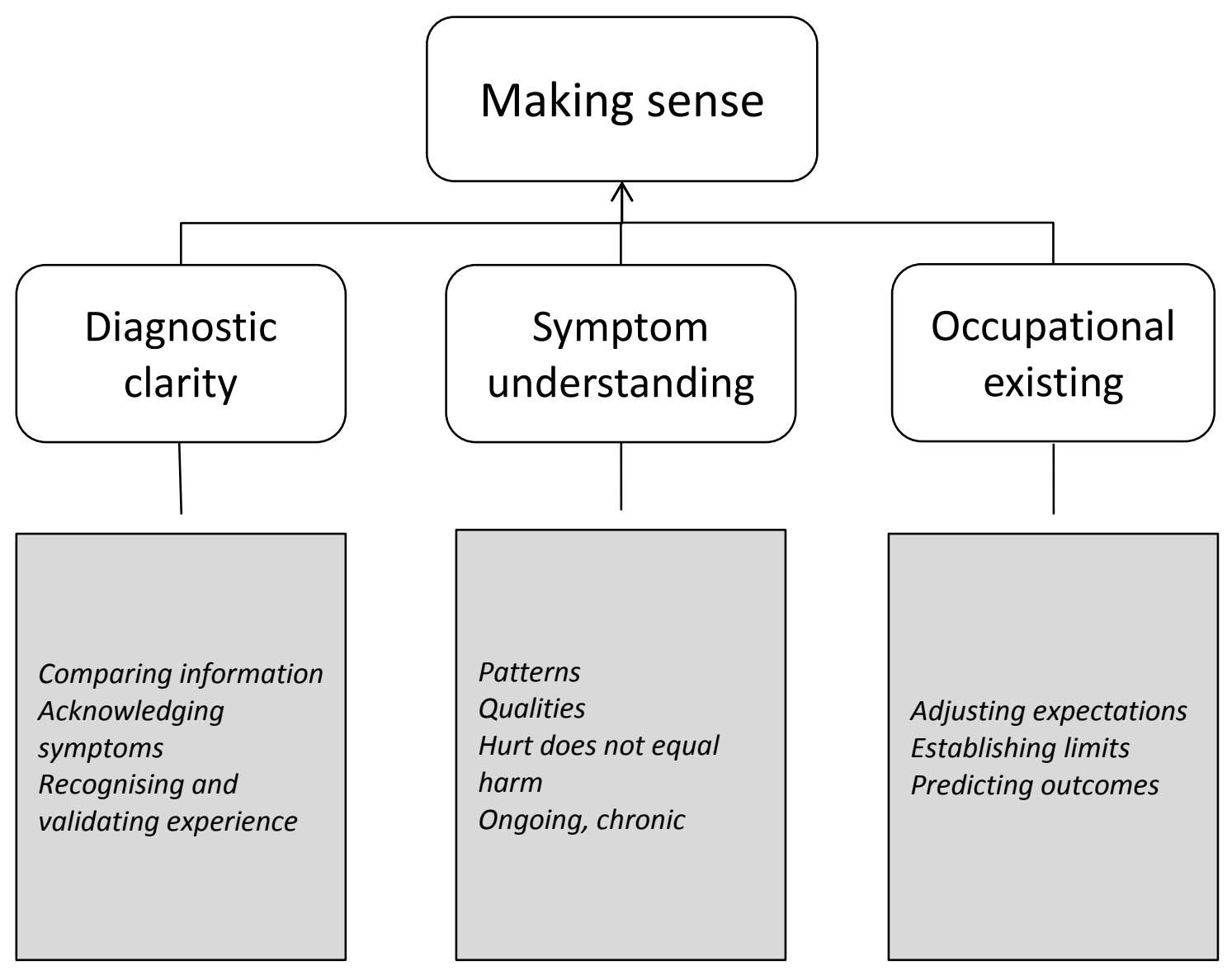

Figure 22. The process of making sense

To be able to devote time and energy to making sense, individuals divert energy from their usual forward life trajectory. This means they experience what participants called "being in limbo-land". The meaning of symptoms is initially unclear, the onset may be insidious, and for many individuals with rheumatological problems, the pain intensity can initially be relatively low. A good deal of time can pass before people decide they need to make sense of their symptoms (Salvadorini et al., 2012). The trigger for starting the process of making sense depends on several factors: the degree of interference with valued occupations and self-concept; pain intensity; experiencing symptoms that are not typical, 
or experiencing concern about the future. Making sense involves diagnostic clarity; symptom understanding and occupational existing.

Note: These three processes occur concurrently and iteratively throughout the period of Making sense of pain.

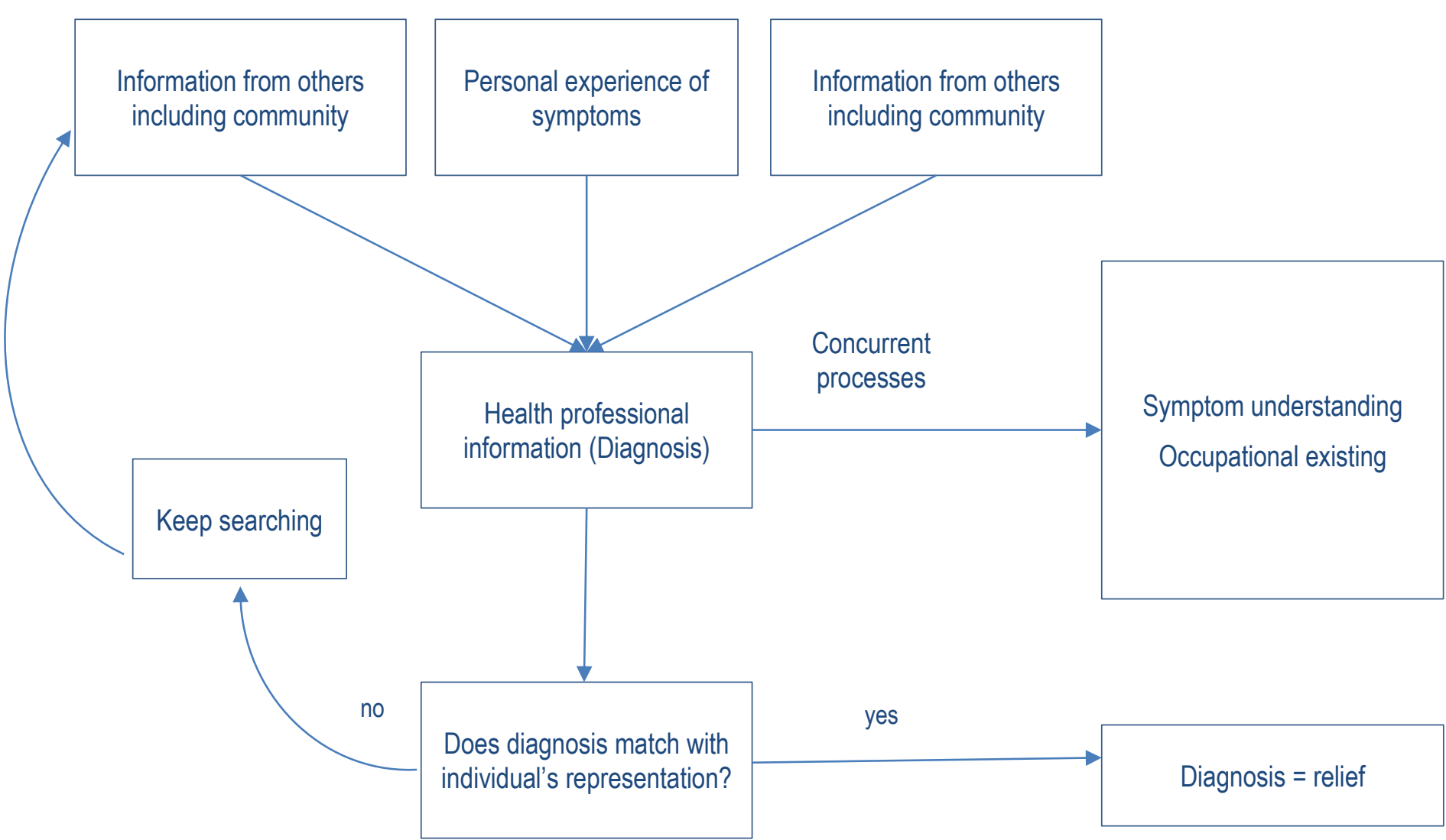

Figure 23. The process of diagnostic clarity.

\section{Diagnostic clarity}

Diagnostic clarity (Figure 23) involves matching information from two primary sources:

personal experiences of symptoms, and the diagnosis made by a health provider.

Information from important others such as family, friends, community, social media and that obtained from books or similar resources also contribute to how willingly an individual 
accepts a diagnosis. Until people receive a diagnosis matching with their personal representation of their problem (a synthesis of personal experience developed from information from other people, the internet, television or other popular media) they may continue to look for a label or pattern of symptoms. This period can be puzzling as they receive different names or diagnoses for their symptoms.

Once a diagnosis is obtained, individuals experience a sense of relief. They may not always understand or acknowledge the reality of living with long-term pain at the time of diagnosis, but believe their symptoms are now known or recognised, and that they no longer need to invest energy in searching for a diagnosis.

Diagnostic clarity involves comparing information from various sources (see Figure 21). It also means acknowledging symptoms. Understanding symptoms that are part of the diagnosis, and those that are not, involves searching for ways to compare internal subjective experiences with those that are vocalised. To acknowledge symptoms, individuals may share their experiences with others, seek verification from other people with the same problems (e.g. peer support groups, online networks), and obtain further information about the diagnosis in order to establish what is and is not expected. Symptoms, that are less frequently socially acceptable in discussion such as pain, low mood, anxiety and intimacy problems (including pain in genitals), may be less readily acknowledged. Clinicians, at this point, strongly influence an individual's response to their symptoms. Advice to stop activities or to use pain as a guide may increase the threat value of pain, and restrict activities. Information suggesting that pain is no longer an indication of harm and that activity is beneficial may support remaining engaged in occupations.

Diagnostic clarity aids in recognising and validating experience. Diagnoses have considerable social valence, that is, by obtaining a name for the problem, the otherwise invisible experience of symptoms is recognised as "real" and allows an individual to claim 
their experience as valid. There is a set of social sanctions and responsibilities that individuals can draw on that in turn provide socially acceptable self-schemas. For example, once a person is diagnosed with rheumatoid arthritis, pain and fatigue are recognised as common problems. As a result, people with rheumatoid arthritis can "give permission" to themselves to stop doing certain activities because the fatigue and pain they experience are now attributable to "rheumatoid arthritis". Instead of feeling guilty for not continuing with normal activities, the diagnosis provides a socially acceptable reason for stopping.

\section{Symptom understanding}

While diagnostic clarity allows for a broad acknowledgement of symptoms and facilitates social validation, the meaning and pattern of symptoms, particularly in chronic conditions must also be established. By developing symptom understanding, individuals begin to establish boundaries and expectations around the patterns of symptoms that they can consider "normal". Once this set of experiences is understood to be within the "normal" range for them, expectations about the effect of symptoms on occupational performance and engagement can develop. This latter process will be discussed under the next process, occupational existing.

In symptom understanding, individuals develop an awareness of the patterns, qualities and ongoing nature of their symptoms. Certain activities and emotions are associated with increases in pain while reductions in pain are associated with others. Becoming aware of these associations is an essential aspect of understanding expectations in different contexts. Factors associated with highly variable pain can be difficult to identify, and this type of pain poses a particular challenge for individuals. Developing a clear understanding of symptom patterns and the effects this has on mood, fatigue and motivation is personal and internal, although may be informed by information obtained from others, for example feedback from a health professional or family member. For people living with painful 
conditions, an especially important aspect of symptom understanding is that hurt (pain) does not equal harm (tissue damage or the threat of tissue damage). This is critical for reducing the threat value of pain as it is experienced in chronic pain (see the definition of chronic pain in Chapter One, p. 1).

While this process is described separately from diagnostic clarity and occupational existing, the three processes interact and influence one another. For example, to develop symptom understanding, individuals engage in occupation. By doing this, they develop an awareness of the pain intensity and quality they may experience, for example, in the morning while getting up and dressed. As symptoms vary from day to day, by engaging in the same set of occupations, individuals develop an internal awareness of typical pain intensity for them and "what it is like" to get up and dressed. Diagnostic clarity informs them of the range of symptoms to notice, and the "acknowledged" pattern that they may experience. Previously ignored symptoms may be identified as part of the disease process or diagnostic label they have received. Similarly, symptoms that may have been identified as highly threatening, for example indicating serious pathology or cancer, may now be disregarded because they can become reframed as a typical aspect of the diagnosis.

\section{Occupational existing}

Occupational existing provides the opportunity for predictive expectations to develop. It is also the way individuals maintain their lives during the time they are making sense. It is by engaging in occupation, however that people begin to understand the effect of symptoms on self-concept. The routines and habits carried out in daily life both provide a means of reinforcing self-concept ("who I am") and of obtaining feedback from others and directly from occupational performance about the effects of pain (and other symptoms) on "what I can do". 


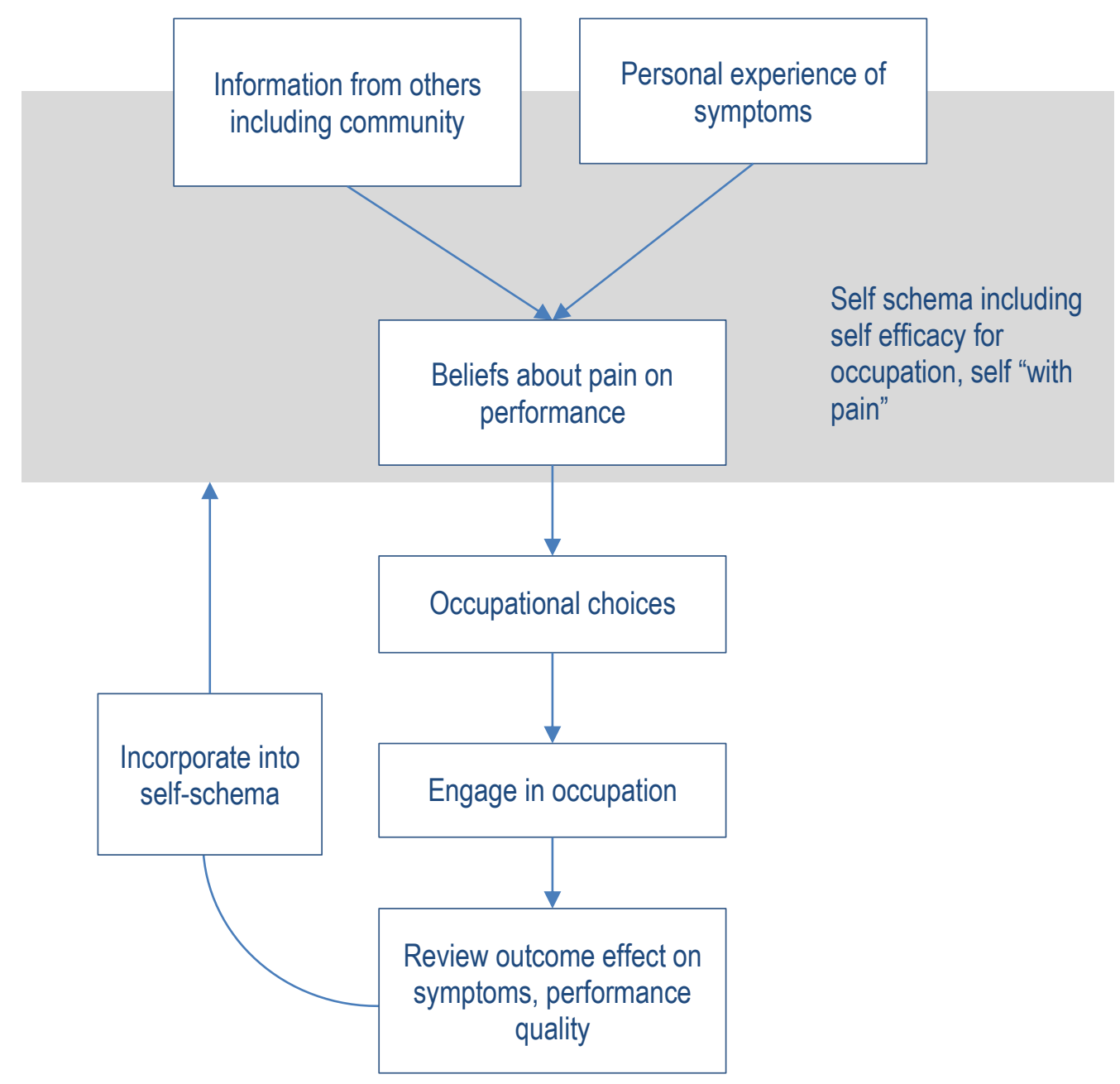

Figure 24. Process of occupational existing

Occupational existing (Figure 24) involves reducing the range of occupations to only those required to maintain status quo during the period of making sense. As noted before, individuals feel they are unable to plan for the future, and they often feel they have insufficient energy to deal with additional demands such as unexpected illness, job change or events. The remaining occupations then provide a way to maintain a positive self-concept "I'm the kind of person who does X", and to obtain information about the effects of symptoms. It is by doing the everyday occupations of getting up, getting dressed, preparing meals, interacting with others, working and engaging in leisure that individuals learn the real effects of symptoms in their lives. For example, 
although a high C-reactive protein is a marker for inflammation, it does not provide any information about "what it is like" to experience a flare-up. The "what it is like" to have a flare-up is learned only by doing the usually expected daily occupations and noticing variations from day-to-day. During a flare-up, many individuals experience increased pain, joint swelling and fatigue. What this means in occupational existing terms is being less able to complete tasks, taking longer to complete tasks, perhaps feeling less motivated to engage in discretionary occupations. Individuals learn to predict that they can do less during a flare-up after having several of these experiences. They may even be able to predict how much less of each task they can do. They begin to establish limits on what they will and will not do, developing prediction rules using symptom fluctuations to predict the effect on performance, for example, "pain of this quality and intensity will settle down overnight", "pain that increases this fast means I need to lie down, or l'll be sick". Occupational existing establishes prediction rules and is influenced by personal experience, beliefs and attitudes, as well as information obtained from other sources such as the internet, health providers, the media, and other people. Some individuals restrict their participation, in which case their occupational existence is narrow, and they and their community identifies them as being disabled. What emerges from this process is a developing, ideographic, and personalised representation of pain for an individual in the context of his or her life and occupations.

An indicative quotation from one participant illustrates this point:

"I know it's only pain, I'm not doing any harm to myself, but I know what my body will and won't do. I've been living with it for so long now, I know when

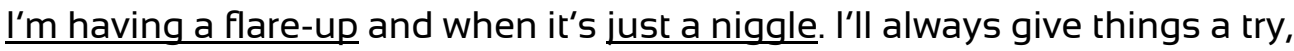
but in the end I'm going to do what I need to do to get things done. I mean, 
sometimes you just need to get on with it and decide for yourself how much you want to let it rule you, some of the things they (health professionals) want you to do for your pain get in the way more than the pain does."

Using the information drawn from diagnostic clarity, symptom understanding and occupational existing in an iterative process, individuals gradually adjust expectations, developing a "self-with-pain" self-concept. Several authors have described chronic pain as a "biographical disruption" that forces individuals to reconsider their past, present and future, often creating a discrepancy between the self-concept before pain and the current self (Asbring, 2001; Richardson, Ong, \& Sim, 2006; Wilson, 2007). The extent to which individuals accommodate chronic pain within the self-concept in a way that retains as much of the past self as possible, without placing unsustainable demands on the future self, influences the degree of acceptance an individual has for their pain. People who cope well with pain develop a self-concept in which the effect of pain is assimilated without compromising critical values in their lives, even though the pain may influence how these are enacted.

The three processes of diagnostic clarity, symptom understanding and occupational existing may be concurrent although it is common for a search for diagnostic clarity to be initiated if not completed before beginning to adjust expectations. This is because diagnostic clarity provides expectations for the future. Individuals who delay adjusting expectations may do so because they hold strong internal rules (for example "should" and "must" beliefs), may be influenced by being offered a complete resolution of their symptoms (so that it may be possible to "return to normal"), or from external influences such as family expectations. In those who cope well with pain, it is only once the prognosis is made clear, and the expectation that chronic pain will likely remain, that individuals can begin to relinquish aspects of their old self-concept. 


\section{Deciding}

This study identified three main factors that influence the process of deciding so that it becomes a positive process. Individuals must have completed the process of making sense. They have diagnostic clarity and know what they are dealing with. They have developed symptom understanding and know the patterns and variations of their symptoms, and also the changes signifying that something new is happening. They can predict and anticipate the effects of their symptoms on occupational performance while engaged in the routine occupations of occupational existing. Making sense as an overall process results in individuals developing an ideographic representation of pain and other symptoms. Individuals factor this representation into deciding because it influences their self-efficacy for engaging in important occupations. The personalised model of their pain

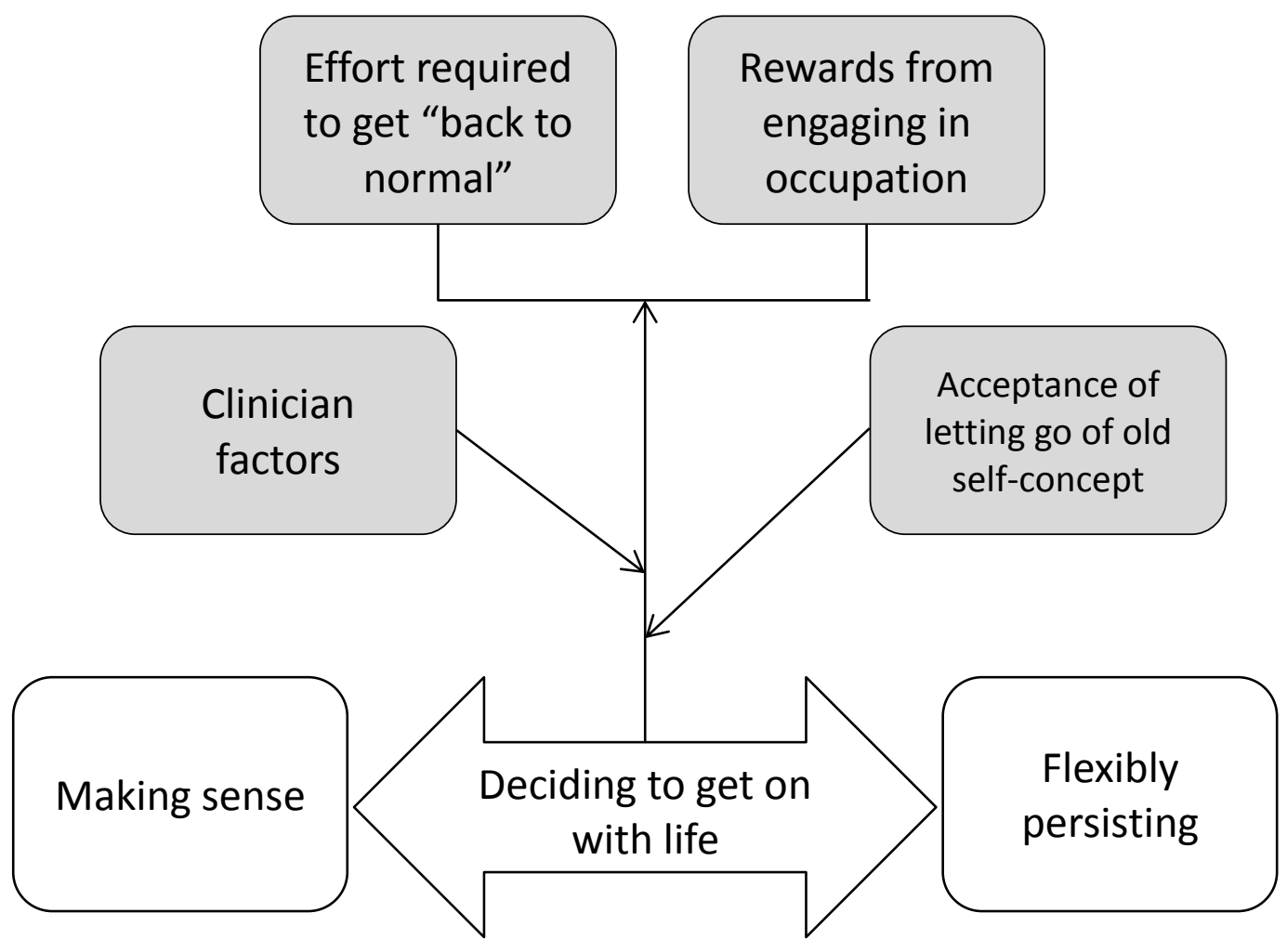

Figure 25. The process of deciding 
helps them predict the pattern of symptoms and plan accordingly. The ability to predict symptom patterns influences self-efficacy because it shows (a) how activities can be carried out irrespective of fluctuations in pain; (b) that flare-ups will eventually subside to baseline, and (c) that the problem is no longer mysterious but is understandable. Selfefficacy is also influenced by the range of coping strategies an individual possesses as I will discuss shortly.

Deciding is a turning point where, when sufficient factors weigh towards deciding to get on with life, the individual re-initiates a forward life trajectory. When insufficient factors are present, the trajectory remains static or continues to cycle through the process of making sense. The factors propelling an individual towards forward momentum are the rewards of (or relief of pressure to) pursue important occupations, and the degree to which individuals have been able to begin adjusting their expectations, and consequently adjust their selfconcept. This is an active decision at a particular time. Participants in my study identified that they were deciding to get on with life as it is now rather than an attempt to recreate their "past self" (Hellstrom, 2001),although the decision may be revisited from time-to-time in light of changes in symptoms or new treatments.

\section{Accepting}

Accepting is a quality of deciding and involves not only making sense, but also occupational drive. In other words, accepting involves weighing up personal information an individual has about his or her pain, comparing this with what he or she has found out from other sources, and balancing this with the drive to continue doing what is important. Accepting means individuals are willing to persist with valued occupations despite variations in their pain and other symptoms, and it also means individuals recognise their pain does not represent a threat. 
Accepting leads to individuals responding to their pain as "it's only pain". In other words, although the pain fluctuates, often depending on activity level, the threat it represents to the self-concept reduces from where it has been in the first phase of making sense. Accepting that hurt does not equal harm is not the same as hearing or being told the same thing.

Accepting represents a personal acknowledgement, often through trial and error, that fluctuations in pain do not mean it will always stay that way or that the underlying condition is changing, and that it is possible to engage in activity because pain no longer represents a sign of harm. This understanding may develop through learning that pain remains whether active or inactive; by experimenting with stopping or starting medication to determine the effect; by seeking opinions from a range of people, many of whom have the same pain problem, or by receiving an explanation that resonates with the individual's own representation of their pain problem. Accepting this view may be at odds with other advice or information given by health professionals. In these situations, the other factors involved in deciding may be more influential than the advice of a health professional. An excerpt from an interview illustrates this:

"When I first got sore knees, the doctor told me I had osteoarthritis. I was quite young...ah...maybe twenty? I was told I should stop playing rugby, stop everything. And I did for a while...ahm... well I kept on cycling because ... well... that didn't seem to bugger my knees as much. But I still had pain, you know. Then I just decided to ...ah... a friend suggested I join in a social rugby team and so I did. I really loved my rugby, and my knees were no worse when I played a game than when I didn't... well... I mean... they hurt more but it was worth it to get back out on the field. And it's only pain it does settle down I'm not doing any damage probably helping if anything because I'm fitter than I was." 
Accepting may be temporarily disturbed during exacerbations of pain, when new symptoms emerge, or in the light of new information that increases the threat value of pain again. If this occurs, the individual may return to the first phase of making sense and seeking to re-establish diagnostic clarity, and symptom understanding during which time energy is directed towards occupational existing. Over time, as the individual becomes more accepting, the frequency with which he or she returns to making sense reduces in frequency and duration. This enables greater engagement in the process of deciding

\section{A trustworthy clinician}

Deciding may also be influenced by the presence of a clinician. Clinicians may either facilitate or obstruct deciding. A trustworthy clinician facilitates by indicating that he or she will "walk alongside" the person with pain, allowing the person to make his or her own decisions, remaining willing to encourage and support the decisions, and by tailoring any treatments to the individual. A clinician who encourages ongoing investigations after the individual has diagnostic clarity, or who recommends further pain reducing treatments when complete pain relief is unlikely, may tip the individuals away from deciding, towards continuing to seek pain reduction or elimination.

Clinicians, who fulfil this role positively (ie trustworthy clinicians), may be present during the process of making sense and may provide diagnostic clarity, support accepting, and may also help to extend the range of coping strategies. Clinicians may be consulted for only short periods of time, and they may not be the primary care clinician (may be from any discipline), but always demonstrate their willingness to do something more. This elevates their input beyond routine practice, engenders their position as trustworthy while their interaction fulfils the role of professionally endorsing flexibly persisting.

Trustworthy clinicians carry out seemingly small actions such as making a telephone call to establish whether an intervention has been helpful; personalising a printed set of 
exercises; obtaining information to answer a question; recording a personalised relaxation CD; sending a brief report to the person as well as the GP. Health professionals, who communicate their willingness to continue to work in partnership with their patients, indicate that although the pain may remain, it is possible to live well and do what is important. They also convey their preparedness to stay involved even if they personally do not have answers. Although they may not agree with the choices the individual makes, they support him or her to be responsible for reviewing and choosing how to live with pain.

\section{Occupational drive}

If making sense is a handbrake on forward momentum, occupational drive is the engine. An individual's strength of occupational drive provides the motivation for deciding. Occupational drive may override any other consideration, including making sense. When this happens deciding may be accompanied by emotional distress, for example, a single parent may feel obliged to continue parenting while still believing that pain indicates harm, consequently experiencing distress during painful flare-ups. For others, however, it is occupational drive that tips the balance towards deciding to move towards the process of flexibly persisting.

Occupational drive represents a "degrees" family within theoretical coding, where the strength of drive exists along a continuum from low strength to high strength. The stronger an individual's drive, the more the individual allocates energy (resource) to pursuing valued occupations. Occupational drive, however, varies for different occupations. When deciding, there must be at least one occupation for which the drive is sufficient to tip individuals towards flexibly persisting.

Occupations come in many forms, and consist of activities that are "active, purposeful and meaningful, contextualized (temporarily, spatially, socially and culturally), and contribute[s] to the construction and expression of self-identity" (Kantartzis \& Molineux, 2011). In 
everyday language as used by the participants in my study, occupations are the "important things that need to be done" in daily life such as driving, being self-employed, caring for children, preparing meals, maintaining the gardens or house, meeting with friends, going to the movies, reading a book.

Occupations enable people to enact their values, fulfil roles, meet others expectations and live out their self-concepts in the context of daily life (Christiansen, 1999). Individuals with a relatively weak self-concept, or one in which their current actual and hoped-for self is enmeshed with their pain have reduced self-efficacy for engaging in activities despite pain (Morley et al., 2005) and, therefore, experience lower occupational drive. Individuals in this situation allocate relatively more energy into finding ways to reduce or eliminate the pain and in managing distress than they do in maintaining valued occupations.

Different occupations provide varying amounts of reward and satisfaction. Occupations particularly valued by individuals provide greater reward than those less valued and increase the drive to remain engaged in them in some way (Persson et al., 1999). Occupational drive provides motivation to find ways to engage in valued occupations. The strength of this motivation depends on the degree to which the various occupations enact personal values. As an example, one participant prioritised her paid employment over her household cleaning because working meant she could remain economically independent, and she could then pay for professional cleaning. Another person cycled or walked to work for fitness rather than driving to work and attending a gym because he could use what he saved on holidays with his family.

Occupational drive is influenced by self-efficacy and by the range of coping strategies and how flexibly these can be applied. I will discuss this relationship when describing flexibly persisting. 
Ultimately, deciding involves making a resolution to do something differently. For people who progress to flexibly persisting, deciding means taking steps to live life as it is, rather than as it might have been.

\section{The processes of flexibly persisting}

The second phase of re-occupying self is flexibly persisting. Flexibly persisting is a mutable state in which individuals allocate their resources to occupational engaging, coping and future planning. The reasons for occupational engaging and coping are to re-occupy self (Christiansen, 1999), thus achieving self-coherence.

Flexibly persisting implies two things: remaining committed to one or more ideal outcome, goal or value, while responding to challenges by finding alternative ways of achieving them. Persistence alone can lead to a restricted range of strategies and a rigid approach to solving problems (Schwenk, 1984). This can be a feature of those who continue searching for a cure for their pain or a return to "normal". Flexibility alone can lead to failing to achieve important outcomes. When both flexibility and persistence are aligned to valued outcomes, motivation and achievement are high (Hassin, Bargh, \& Zimerman, 2009).

\section{Occupational engaging}

Occupations are the stuff of everyday life, the activities that humans engage in as part of "being" and "doing" life (Hocking, 2009; Law, 2002; Pierce, 2001; Reed, Hocking, \& Smythe, 2010). Occupational engaging entails more than occupational existing, which occurs during making sense. Occupational engaging involves actively pursuing occupations of value to self-identity, but that also contribute to an ongoing sense of purpose and meaning.

Self-identity and occupations are inextricably linked. Occupations provide "purpose in the moment" (Christiansen, 1999). That is, the occupations of daily life give continuity for an 
individual over the course of a day. Occupations can be described as "goal-directed activity in the context of living" (Christiansen, 1999), thus providing motivating action as people imagine the effect of goal achievements on their "self". The following examples from participants demonstrate this relationship:

"I just have to do these things: I'm a mother, there's no-one else."

"If I don't keep playing I lose something that's part of me"

"What's the point if you can't do what makes you feel like yourself?"

"I'm the only one who knows how to do the training, so I keep going"

Individuals use occupational engaging to bridge between the current self and a future state. For example, one participant said: "I always get up and have a shower and put on my makeup, even on a bad day. I might feel like I can't handle the pain, but when I'm up and about I feel more myself." The outcome of completing the morning routine provides confirmation for this individual that, despite the current state being "a bad day", it is possible to be the "ideal" self.

To carry out this self-confirmation, individuals use what they know about their abilities and limitations (derived from making sense developed during the first phase of this process); accommodating their functional limitations and enhancing their abilities, so they can become their "ideal self". Occupational engaging both provides appropriate feedback and enacts self-identity. There is an interaction or feedback loop between occupations and self-identity. The feedback obtained from occupational engaging provides evidence of the current self; discrepancies between the current self and ideal self then guide "corrective action", while the ideal self provides motivation towards engaging in occupations that enact this self-image (Figure 23). 
There are three broad types of occupations, based on the purpose or function fulfilled by them.

1. Some are discretionary and are clear choices made by individuals as part of selfexpression, social identity, or for leisure and fun. Occupations such as these are choosing to cycle or walk to work, engaging in hobbies and meeting socially with friends.

2. Some occupations are obligations or activities individuals feel they must or should do, so they meet their own or others' expectations. These occupations include mowing the lawns, clearing the guttering, earning money through paid work, the range of occupations involved in being a parent or partner.

3. Finally, there are occupations to support health, such as injecting medication, meditating, obtaining information about their health condition, and actively developing networks and supporting others through online networks. A specific occupation to support health is "finding and using ways to get on with life". This can involve seeking pain reduction, but typically focuses on maintaining engagement in what is important to the individual. 


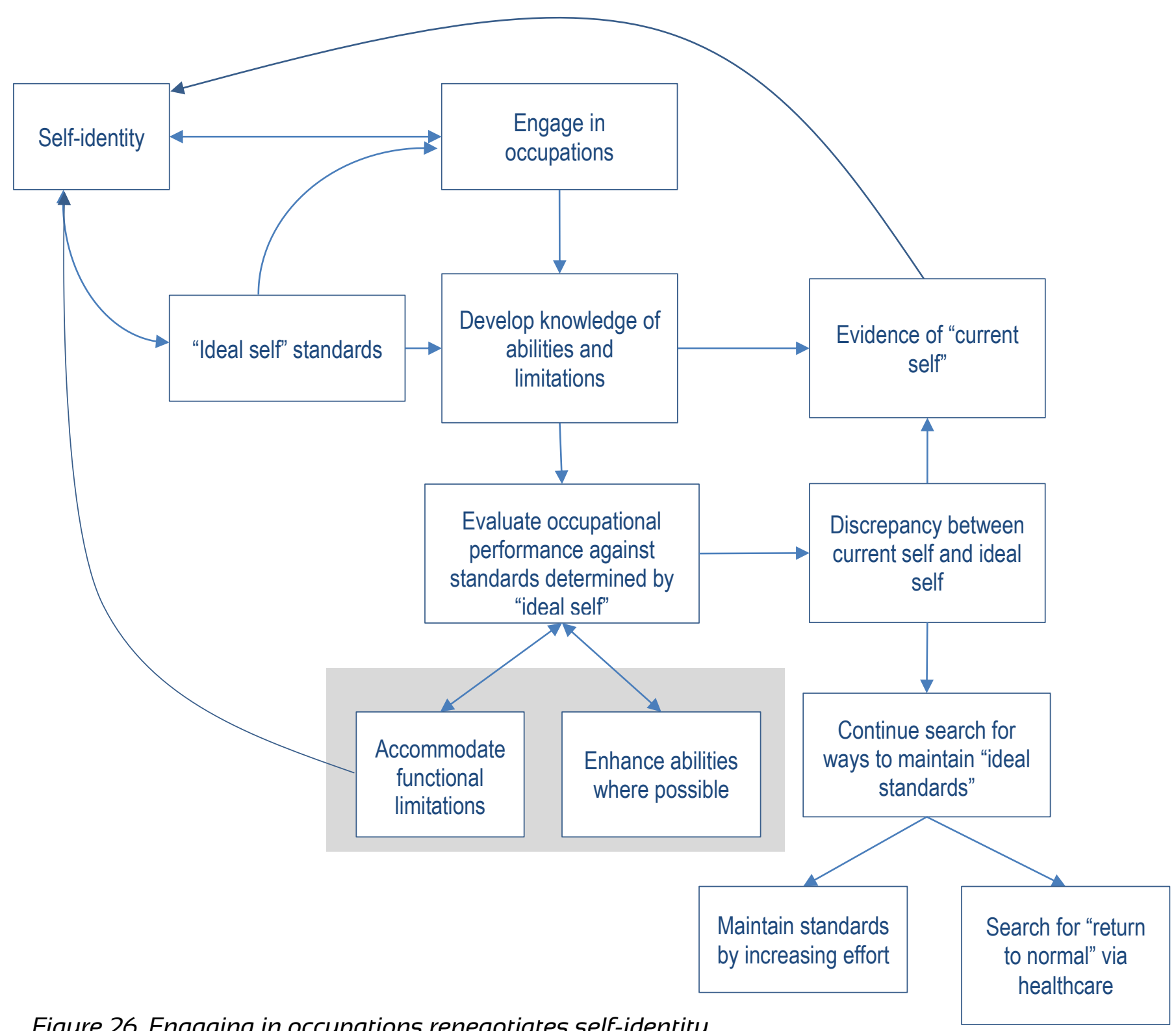

Figure 26. Engaging in occupations renegotiates self-identity

Together, all three forms of occupation enable individuals to express and create a selfidentity that provides continuity and a sense of coherence, and upon which future plans can be developed. They re-occupy a newly-revised self-concept, and in doing so achieve self-coherence. The major distinction between occupations engaged in prior to pain onset is an increased emphasis on occupations to support health. Health-related occupations become part of the individual's self-identity, but, importantly, are incorporated into selfidentity as "part of living well" rather than defining self-identity. People living well with pain 
do not identify primarily as "pain patients", but see themselves as people who happen to experience pain.

\section{Coping}

Coping enables individuals to engage in their occupations in a way that aligns with their self-identity. By this, I mean that individuals use "whatever helps" so they can participate in occupations that both express and inform their self-identity (Figure 25). Coping in this model is both a set of strategies and the process of using them. Coping involves momentby-moment decisions about what is important and how it can be achieved within the context of an individual's life.

The strategies employed by individuals are not easily dichotomized into "good" or "bad, "active" or "passive" (as in Brown and Nicassio (1987); Day et al. (2012); de Ridder and Schreurs (2001); Haythornthwaite and Heinberg (1999)). Individuals select different strategies depending on context, and they employ the strategies in a flexible way. Contexts vary by the time of day, day of the week, the people present, the physical environment, personal expectations and sociocultural values. For example, to maintain work, one participant described it thus:

"I plan my day. What does it matter if I ask for help, or take a nap, or rest during the day, if I can meet my client first thing in the morning because that's when I'm feeling better, then I can do that. That's why I decided to be self-employed, so I can. I will push myself to be there for a client, but crash later. Sometimes I'Il decide to phone them up and change our time because it works better for me on that day. I couldn't do that if I was employed by somebody."

This person uses a broad range of strategies (underlined) so she can maintain her employment. She selects different strategies depending on her priorities, and has reconceptualised what she believes is acceptable work behaviour so that she can accept 
the need to change an appointment to give her best to a client, when at other times she prefers to push herself to be there. By having many different coping strategies at her disposal, she can choose the approach that will ensure she fulfils her priorities in a range of contexts.

Coping is best conceptualised in functional and contextual terms. Accordingly, the outcome obtained by using them, in the occupational context of an individual's life, determines their utility. Coping enables occupation in people who cope well with chronic pain, and engaging in occupation generates the need to cope. When an individual possesses a large number of coping strategies, and can apply these in a flexible way, selfefficacy for engaging in occupation is high. Self-efficacy for coping depends on making sense, and in particular, on accepting that pain does not mean harm.

There is a great deal of "behind the scenes" work in coping. Coping involves modifying self-identity schema so that strategies can be employed without experiencing the distress that is usually associated with discrepancies between the current self and the ideal self (Figure 26). For example, when using pacing (that is, working for a pre-determined period of time or "chunk" of activity), an individual may initially violate his or her sense of self as a person who "never leaves a job unfinished" and experience frustration. If he or she can modify a self-schema to prioritise an alternative one such as "If I am a good parent, I will spend time with my child: the housework matters less than spending time with my child", he or she can alleviate negative emotions. The process of modifying self-identity schema is at the heart of cognitive behavioural therapies (Markus \& Wurf, 1987). Individuals who are flexibly persisting renegotiate their self-schema through coping and occupational engaging by finding ways to meet their underlying values. In ACT terms, this ability represents psychological flexibility (Hayes et al., 2006; McCracken \& Gutierrez-Martinez, 2011; McCracken \& Morley, 2014). 
It is unsurprising that people with chronic pain employ a wide range of strategies if the concept of coping is reconceptualised in functional and contextual terms. In functional and contextual terms, coping strategies are more or less useful depending on the context and purpose for employing them. No single approach provides an optimal outcome in every situation. Thus, strategies are used in a flexible way: if a particular strategy is unavailable, or the situation/context means (in the perception of the individual) that using

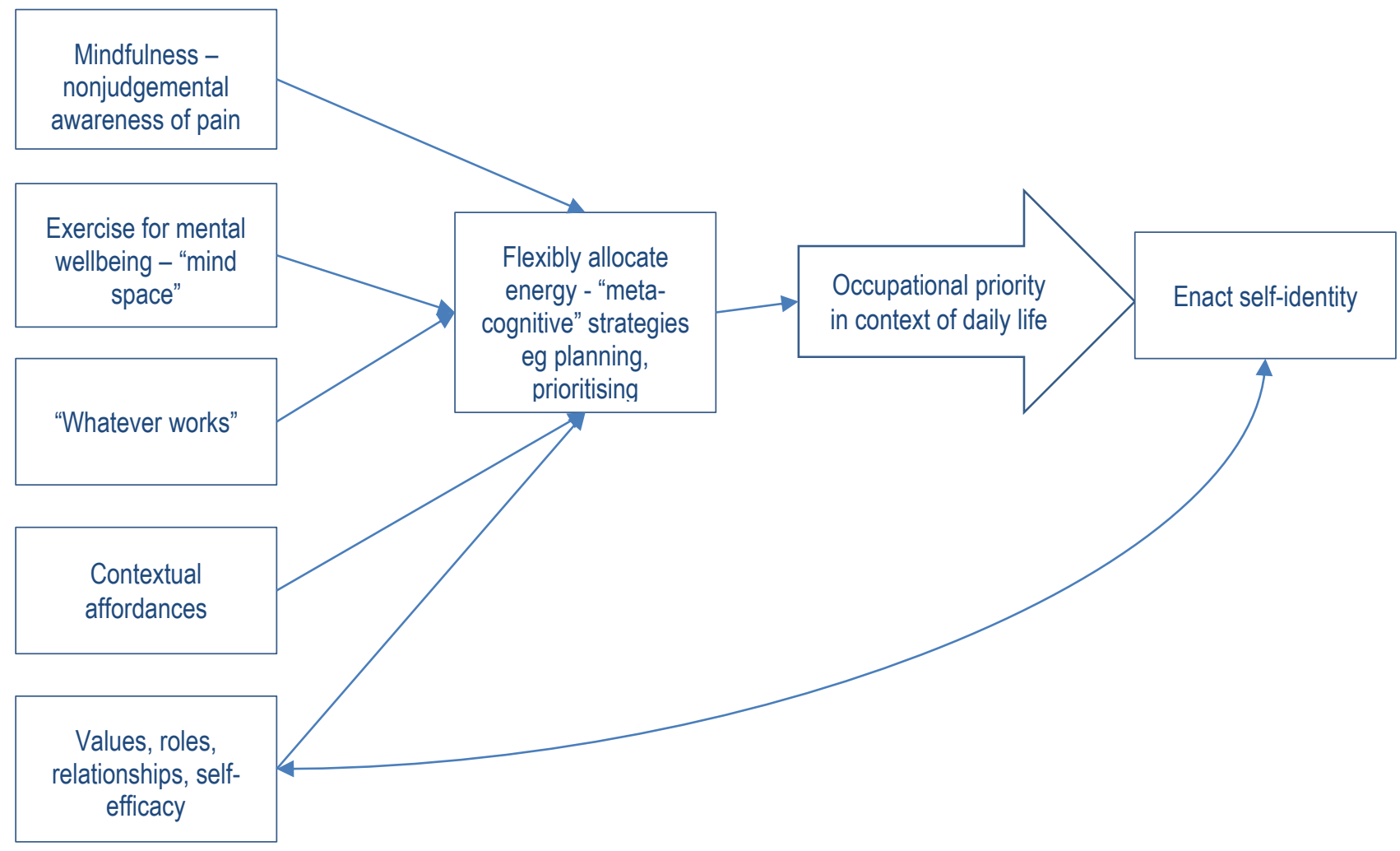

Figure 27. Flexibly persisting.

a strategy would be inappropriate, an alternative one can be employed. This also explains why individuals who cope well endorse many strategies considered by Folkman and Lazarus as passive and unhelpful (Folkman \& Lazarus, 1980). Resting, in the example above, enables the person to participate in work, although it is often considered passive and contributing to disability (Kraaimaat \& Evers, 2003). The boom and bust pattern of 
activity where increased activity is followed by periods of rest to recover is a common pattern amongst participants in my study. The boom and bust pattern has often been associated with gradually declining levels of activity although recent studies suggest it may be more adaptive than originally thought (Kindermans, Roelofs, et al., 2011;

McCracken \& Samuel, 2007b). Pacing is usually the technique recommended to counter a boom and bust pattern, but suffers from a lack of conceptual and theoretical clarity (Nielson et al., 2013). People who cope well with chronic pain regulate their activity and rest patterns so they can engage in what they perceive to be important at that time and in that context.

Despite finding that people use a range of coping strategies at different times and in different ways, there were some consistent approaches employed by all participants in this study. Three main groups of strategies emerged: Mindfulness, Exercise and Whatever works

1. Mindfulness describes nonjudgemental awareness of pain. Those coping well with pain are aware of their pain, but not bothered by it, as distinguished from ignoring pain or distracting away from pain. Mindfulness involves being aware of pain in various parts of the body, noting its quality, intensity or spread, yet remaining dispassionate about the experience. Mindfulness is used both deliberately and as a routine habit and emerges once individuals accept that hurt does not equal harm. Nonjudgemental awareness means individuals acknowledge the presence of pain, but in the context of personally-relevant occupations, pain lacks emotional power. One participant described the pain as "irrelevant", another called it "just noise", and another described being "not bothered about it, it's not important". Importantly, individuals do not attempt to ignore, distract themselves from or control their pain. 
Pain is present, but not highly prioritised when weighing up the factors relevant to engaging in occupation, except when planning an overall schedule.

2. Exercise is used in all its forms, including dance, walking, cycling, swimming, rugby, yoga. At times exercise is undoubtedly a valued occupation, usefully classified as supporting health or discretionary. However individuals use exercise as a particular form of coping that enables occupation. Occupations such as gardening or housework are not identified as coping strategies because although they can be vigorous, they do not enable occupation but are instead occupations in themselves. Underlying purposes of exercise are to maintain a "healthy body" (maintain strength, range of movement, cardiovascular fitness), and for mood/stress management. Exercise may incorporate specific exercises to prevent deformity, maintain or extend range of movement, develop or maintain strength or to relieve pain (mainly stretching), although adhering to a daily regimen of exercises is less frequently found than using them intermittently, most often during painful exacerbations. Routine exercise more often involves repetitive and rhythmic movement patterns such as walking, cycling, swimming or dance, but may include attending the gym or playing a sport. For some, exercise is a primary occupation in itself and is a reason to get on with life. One participant described it this way:

"I didn't want to stop my rugby, but I thought I should because the doctor said I should be careful not to overdo things and when you play rugby you do put yourself out there. After a few years of sitting on the side-lines I decided to get back into playing again because it's who I am. If I don't keep playing I lose something that's part of me. So I started going to the gym and running so I could play rugby again, and I made the Masters team." 
3. Whatever works incorporates an extensive number of strategies used to enable occupation. These include: cognitive strategies (e.g. positive self-statements, "gritting your teeth", reality testing); communication and establishing boundaries (e.g. assertive communication, asking for help, delegating); relaxation (e.g. breathing, rest, progressive muscle relaxation, differential muscle relaxation); activity management (e.g. planning, prioritizing, breaking a task into "chunks", evaluating the day/week/month's activities); passive modalities (e.g. coloured light machine, osteopathy/chiropractic/physiotherapy, TENS, heat); medications (e.g. prescribed, and taken mainly in a time contingent manner, but occasionally taken as needed); spirituality (e.g. prayer, meditation); gadgets and assistive equipment (e.g. appropriately adjusted office furniture, lumbar roll, spinner on the steering wheel).

These strategies do not pattern out into separate sub-categories, in part because their use is less regular than either mindfulness or exercise and because they may be used to achieve a range of different outcomes. For example, asking for help may be variously used to: ensure an individual can complete a valued activity; elicit caring from another; enable time out so another occupation can be carried out; foster interpersonal relationships; reduce fatigue or to increase confidence. The important characteristic of these strategies is the function they perform for the individual in the context of flexibly persisting. They are not, in isolation, adaptive or maladaptive, but rather must be viewed in light of the circumstances in which individuals use them.

Medications are also a form of coping used by people who are flexibly persisting. Medications are, however, used primarily to manage the disease process rather than to reduce pain, such as anti-inflammatories to reduce swelling and stiffness, or diseasemodifying drugs to reduce disease activity. Individuals hold conflicting views about taking 
medication, with many trying medications for a period of time but eventually stopping them. The reasons for stopping medications were because of the minimal effect they had on pain, not wanting to live with side effects, or because individuals preferred to manage without drugs. Some use analgesia only on a prn or "as needed" basis, despite acknowledging limited effectiveness. This is to support the individuals to engage in a particular occupation, for example, taking paracetamol or ibuprofen before embarking on unusually physically demanding occupations.

Individuals often use coping strategies to "recover" from their valued occupations, so that relaxation techniques, for example, might be used to help get off to sleep after being out at a function, or resting might be used the day after a busy day.

Coping has the dimensions of flexibility, open-mindedness, noninvestment, and context. Flexibility refers to the range of strategies and the ways in which individuals use them. Open-mindedness refers to the attitudes individuals have towards trying anything that may help. Noninvestment refers to the way individuals continue doing what is important without waiting for an approach to provide a complete cure (in other words, not holding out too much hope that any intervention will be "the cure"), and context refers to the time, place, and purpose in which individuals use coping strategies.

\section{Future planning}

During flexibly persisting, individuals have clarity about what is important in the context of their life, they are persistent in pursuing important occupations, and they are flexible when tackling the challenges they encounter. They accept the reality of their pain, but the pain no longer holds the threat value it had when it first began. They are willing to experience pain if the rewards arising from their important occupations are greater than any adverse effects of increased pain. Rather than managing pain, people who cope well with pain, manage their lives, goals and actions. Being in this phase means they can embark on 
future planning with pain rather than attempting to return to "normal". Future planning can only occur when the world and self are once again comprehensible, manageable and meaningful. When this happened, individuals have sufficient self-efficacy to have positive expectations for their success in occupations, they are motivated to cope (the world has meaning), believe that the challenge can be understood (comprehensible), and that they have the resources to do so (manageability).

Future planning in this context implies a future where pursuing pain relief or a cure for their health problem is no longer the main concern. Instead, the interest shifts to how to continue living a meaningful life despite the presence of a chronic disease.

\section{The model as a whole}

The grounded theory of living well with chronic pain explains why and how some people are able to remain resilient and continue with life goals despite having sustained a significant threat to their sense of self. I contend that the main concern of people experiencing chronic pain is to find a way to return their world to something that is understandable, meaningful and manageable, and to feel that they fit and can contribute positively. Although individuals may not recognise that they are re-occupying self during this process, in effect, this is what they achieve once they are flexibly persisting. Reoccupying self involves gathering information and skills, examining what is and is not important, applying knowledge and skills until the new self-concept feels comfortable.

A useful image is the metaphorical "black dog": initially the dog invades a person's home, dominating every part, wilfully destroying cherished items around the home, and even sitting on the person's lap so that all they can see is the dog. It is only once the person puts it into its place (perhaps the dog kennel outside?) and begins to re-occupy the home that they can feel comfortable and able to repair some of the damages done. Things will 
never be as they were before the dog arrived, but room can be made for it, and life adjusted so that it continues with meaning.

\section{Contributions to knowledge}

Despite the enormous investments individuals and organisations have made in investigating the problem of chronic pain, there are many aspects that have yet to be understood. This thesis makes several contributions to knowledge about chronic pain in the seldom-studied population of those who remain resilient and have learned to live well despite ongoing pain. The main contributions to knowledge are:

1. An explanation for the processes people who cope well with chronic pain follow as they resolve their main concern;

2. An explanation of the way self-identity is adjusted to accommodate chronic pain as a part of, but not defining, the self-concept;

3. The importance of occupation as a key factor in providing motivation for individuals to develop coping;

4. An understanding of the process followed by individuals when making sense;

5. The need for clinicians to individualise treatment and support people with pain as they develop their own ways of achieving self-coherence, and some ways to demonstrate support;

6. An understanding of the process of flexibly persisting as individuals are reoccupying self;

7. The shift in focus from "limbo land" and occupational existing to occupational engaging and future planning with implications for treatment goals;

8. The value of a functional contextual perspective on coping used in daily life.

While some points have been identified with respect to other populations of individuals with chronic pain, this study has shown the relationships between these factors in people 
who are naïve to cognitive behavioural approaches for managing pain. Furthermore, this study has shown that individuals who cope well routinely use both mindfulness and exercise as when coping, along with flexibly using a repertoire of strategies, some of which have previously been categorised as maladaptive or passive. This supports the value of a functional contextual perspective on coping: it is not just "what you do", but also "the way that you do it", and the "why you do it" to achieve a desired outcome (Van Damme et al., 2008).

People report living day-by-day when they replace their life goals with the objective of finding a cure or complete relief from pain (Hellstrom, 2001). They experience difficulty planning for the future because of their need to find ways to avoid exacerbating their pain, or being unable to participate in activities because of pain exacerbations (Morley et al., 2005; Sutherland \& Morley, 2008). Self-efficacy to re-engage in occupations and roles previously highly valued is considerably reduced, particularly in individuals with anxiety and depression (Affleck et al., 2001 ; Asante, Brintnell, \& Gross, 2007; Asghari \& Nicholas, 2001; Meredith, Strong, \& Feeney, 2006; Nicholas et al., 2009).

Low self-efficacy, competing goals with the aim of abolishing pain rather than accommodating it, and having difficulty making sense of pain or predicting its daily fluctuations make it difficult for some individuals with chronic pain to contemplate the future in case their pain interferes. Additionally as Kindermans, Huijnen and colleagues (2011) point out, self-regulation theory predicts that individuals who experience selfdiscrepancy (a distance between their current actual self and ideal self) because of anticipated pain exacerbation or current pain severity will demonstrate low mood and distress as well as reduced activity levels (Kindermans, Huijnen, et al., 2011; Nes, Roach, \& Segerstrom, 2009). Consequently, individuals may not actively engage in activities that may lead them towards their ideal self if those behaviours could potentially increase their 
pain (Sauer, Burris, \& Carlson, 2010), or if these behaviours conflict with deeply held beliefs about the self (Karoly, Okun, Enders, \& Tennen, 2014; Meyer, 2010). From my study, individuals who cope well with pain appear to retain, or develop fairly quickly, a sense of self that incorporates pain and the need to use different ways of achieving a goal: this may be an avenue to explore in the early phases of living with chronic pain.

The primary reason for individuals effectively coping is so they can achieve self-coherence and re-occupy self. The contribution of occupation to provide motivation for coping is critical. Goal-setting, problem-solving and many of the coping strategies endorsed in chronic pain management programmes are predicated on the idea that individuals already have identified occupations to which they wish to return. This may be a misplaced belief given that many individuals experience demoralisation and loss of future focus while searching for pain relief. By providing treatments tailored to making sense, deciding or flexibly persisting, interventions may be more readily accepted and clinicians may find their work more rewarding.

Within this chapter I have detailed the processes involved in resolving the main concern of people who experience chronic pain. The model has been derived from empirical research in which repeated incidents provide indicators of underlying concepts. The theory fits to explain the processes of re-occupying self in people who live well despite their ongoing pain, and it works to provide ways in which clinicians and people with pain can understand what is going on. It has immediate relevance to clinical practice, but also offers some intriguing new hypotheses for further research. Within the next chapter I examine literature relevant to my findings to position and inform the theoretical propositions I have made 


\section{Chapter Six - Integrating the literature}

The empirical findings and theoretical concepts identified in my study, as I have described in the previous chapter provide a greater understanding of how and why some people identify that they live well despite having chronic pain. Within the cognitive behavioural tradition in chronic pain management, "adaptive" coping strategies such as activity management are encouraged while "passive" strategies such as resting are discouraged (Haythornthwaite \& Heinberg, 1999; Snow-Turek et al., 1996). Participants in my study successfully employed strategies across the spectrum, yet identified themselves as well and were neither distressed nor disabled by their pain.

The current view of coping with chronic pain is incomplete because it ignores two important points. The first is that coping does not occur outside a context. Coping strategies cannot be dichotomised without simultaneously considering why and how they are used. The second is that there must be a reason for coping: a goal or rationale for an individual to decide to adopt new ways of doing things, especially if pain reduction is not the primary aim. The way coping is construed within current chronic pain management practice, may need revising. Coping with chronic pain is more than adopting a particular set of strategies.

I propose that the process of re-occupying self is a critical process for people adapting to chronic pain. By redeveloping a self-concept that incorporates pain rather than rejects it, individuals can understand their world and self so that life is meaningful, understandable and manageable. Individuals that achieve this successfully have achieved self-coherence. Coping is then repositioned as enabling individuals to occupationally engage, or do the things that express and reinforce self-identity. By making sense and flexibly persisting, 
chronic pain has less threat value and no longer defines who the person is, nor what they do. Coping is thus a process of finding and using strategies to enable a new identity to develop.

Within this chapter, I will position my findings and integrate existing research to show the unique contribution my research makes to understanding the ways individuals learn to live with chronic pain. I explained in Chapters One and Three that classical grounded theorists complete a comprehensive literature review only after empirical data collection and analysis. This minimises researcher preconceptions, thus supporting abstract conceptualisation from the data rather than inadvertently fitting the data to existing theory. Delaying the literature review also allows for unexpected findings from the data that may make any prior review redundant. The literature plays a greater role after data analysis by developing the emergent theory, providing context and extending the discussion. I have organised this chapter to firstly discuss several models of coping, before I examine identity and self-concepts, occupation and meaning, diagnosis and clinician-patient relationships, and the therapeutic approach that is most strongly aligned with a functional contextual perspective of coping. Within this chapter, my findings are integrated, and some of the implications considered.

\section{Coping and identity}

A rich body of literature exists to explain adaptation to, or coping with stress, and there is little doubt that having a painful health condition represents a powerful stressor. Livneh and Martz (2007), in a recent chapter introducing coping theory and research, identify five broad conclusions about coping, saying:

(1) Coping strategies differ between individuals and change over the course of a lifetime; 
(2) Coping strategies are more or less effective, depending on the stressor, and characteristics of the stressful situation;

(3) The effectiveness of coping strategies depends on good "fit" between the individual and his or her environment, and the strategies used to cope with the stressor;

(4) To be effective, individuals need to be able to draw on a flexible range of coping strategies, and

(5) Overall, coping strategies mediate between a stressful situation and the impact of that stressor on the individual's experience (Livneh \& Martz, 2007, p. 9-10).

There are various themes with respect to defining effective coping, but the general thrust is transactional and self-regulatory: individuals appraise their situation and experience an emotional response that subsequently engenders a behavioural response. A feedback process enables ongoing appraisal and subsequent response adjustment.

Coping is, therefore, about adjusting to a stressor, so the impact of the stressor is reduced. I propose that, when learning to live well with chronic pain, coping is about adjusting the self-concept so that the things that bring meaning to an individual remain despite the ongoing presence of a stressor. The reason for learning to cope and using a range of strategies is to regain a sense of coherence about self and the world.

Coping theories, however, rarely explicitly consider why individuals adopt a particular set of coping strategies. While models discuss approach and avoidance, and perhaps envisage a return to homeostasis as primary motivational drivers, these are seldom integrated with overall life meaning or the reason for coping. This grounded theory describes a self-regulatory process in which coping enables occupational engaging. Occupational engaging embodies and provides feedback on aspects of the self-concept which in turn allows the individual to modify self, and ultimately, to re-occupy self. The choice of occupation and coping strategy maintains the 
integrity of the self-concept by integrating a self-with-pain schema into the self-concept that allows valued aspects of self to be expressed.

I will outline several themes about coping, pointing out both similarities and differences between them and those obtained through my research. I begin with general theories of coping, and move into those specific for coping with pain.

A brief reminder of terminology: coping refers to the process of adjusting to a stressor, coping strategies refer to the techniques used by individuals within the process of adjustment, while in lay terms, coping can mean the outcome of having used strategies and achieved a positive end, particularly where the coping strategies become habitual. I use the term living well to indicate this latter meaning.

\section{Identity, self-concept and illness}

Identity and self-concept are present in the literature of coping (Irvine, Davidson, Hoy, \& Lowe-Strong, 2009). Baumeister (1997) writes extensively about self, self-concept and identity, and defines self as "the direct feeling each person has of privileged access to his or her own thoughts, feelings and sensations" (p. 681). He refers to self-concept as "the totality of inferences that a person has made about himself or herself" and incorporates an individual's own knowledge of social roles as well as personal schemas. Lastly, he defines identity as "the definitions that are created for and superimposed on the self" (p.682). He goes on to say that identity consists of an interpersonal aspect (roles and relationships), a potentiality aspect (a concept of who the person might become), and a values aspect (a set of values and priorities) (Baumeister, 1997, p. 682). Illness and disability impact upon all three aspects of self as I will show below. The strategies employed to cope with a threat to aspects of self are coping strategies, while successful coping enables continuity of the sense of self. 
To begin discussing how the coping, illness and identity are related, Charmaz (2002) believes that habits "of thinking, feeling and action that people invoke without reflection" (p. 315) enable people to respond efficiently and economically and represent a link between self-identity and the social world. She considers that an individual's self-identity, and the boundaries within which a person functions, develop over time through habitual preferences, emotions and thoughts towards themselves, others and the community. Charmaz (2002) points out that ill health requires changes to usual habits as individuals deal with ordinary life, such as how to clean the house, manage personal hygiene, and go to work. Understanding the implications of a diagnosis on the self-concept takes both time and opportunity to explore the "habitualised notions of self" (Charmaz, 2002, p. 316). Charmaz explains that when changes to everyday habits occur as the result of ill health, tensions arise between the past self-concept and new behaviours and self-appraisals (Charmaz, 2002; Charmaz, 1995). Over time, new habits and self-appraisals eventually develop into a new sense of self, in response to both feedback from other people within the social context, and personal judgements about the situation, experience and interactions (Charmaz, 1995). The self, as Charmaz argues, is continually changing but is in particular flux during the early stages of learning to live with chronic ill health (Charmaz, 2006c). She points out that when people remain resilient despite living with poor health, their bodies may change, but their "selves" remain (Charmaz, 2002; Charmaz, 1995, 2006c). She does not, however, examine the process of integrating new habits and selfappraisals in detail.

Processes involved in maintaining continuity of self are explored by other commentators. Faull and Hills (2006) elaborate on the concept of self and identity, saying that the individual must perceive their core self as constant and continuing, despite changes present in every other sphere of life. The work of adjusting to, or coping with ill health involves identifying those parts of the self that can and do continue despite these 
changes, thus allowing those aspects that change to be integrated without sustaining irrevocable blows to the sense of self (Faull \& Hills, 2006, p. 732). Harris, Morley, and Barton (2003) suggest that roles may change over the course of an illness, but attributes or "internalised cognitive representations of the self in relation to others" continue albeit in different forms (p. 363). The ways individuals maintain these representations are not clarified by these authors.

There are other formulations of the process used by those experiencing illness and/or disability to adjust to a new self. Oliver (1996) defines three characteristics that define an individual as "disabled": (1) the presence of an impairment; (2) the experience of externally imposed restrictions, and (3) self-identifying as a disabled person (1996, p.5). Points two and three are particularly relevant because they are self-representations while point one suggests some sort of social validation is required. Watson (2002) argues that people with disability "go through a process during which they negotiate their lives in such a way as to be as ordinary as possible, and so retain some contact with desired life-worlds" (p. 513). Salick and Auerbach (2006) studied individuals with a disability and found five stages involved in adjusting to disability, with the first two being Apprehension and Devastation. Apprehension characterized the initial stage, with participants becoming aware that their bodies were not functioning well; and a sense of unease, particularly with respect to "performing adequately in social settings" because of changes in physical abilities (p. 1026). Devastation and Diagnosis occur together. These stages refer to the participant's experience of receiving a diagnosis, recognizing the impact of this on their ability to participate in usual activities, and leading to what the authors describe as "psychic pain" and "hopelessness" (p. 1028). Individuals in Salick and Auerbach's (2006) study then identified a stage designated as Choosing to go on, when they decided not to miss out on connections with the world, found an inner strength and developed plans to find ways to get around their problems. This was followed by Rebuilding when individuals 
reclaimed the physical body, synthesised a support system, and found personal meaning in finding hope and using humour. The final stage in Salick and Auerbach's model was Integration, when people were moving forward, giving back, and experiencing a sense of purpose and meaning (Salick \& Auerbach, 2006).

If aspects of self are threatened by illnesses that will ultimately resolve, what happens when health problems are chronic and the "ideal" or past self-concept conflicts with the existing self and externalised self-identity? Self-discrepancy theory (Higgins, 1987; Higgins, Klein, \& Strauman, 1987) has been used to explain existential and emotional responses to stressors affecting self-concepts. The theory has been applied widely in mental health as well as physical health, and next I will discuss selected findings in chronic pain.

\section{Self-discrepancy theory applied to those with chronic pain}

In self-discrepancy theory, individuals are thought to hold beliefs about themselves based on what is (actual), beliefs about how one wants to be (ideal), and beliefs about what others think one should be like (ought) (Higgins, 1987). When there are discrepancies between these three sets of beliefs and an individual's understanding of what others believe about them, individuals experience distress, sadness, and motivation to reduce the discrepancies. The process of reducing self-discrepancy is part of coping. Hellstrom (2001) found that individuals experience the body as separated from the self, not belonging to the self in the way it had before the pain began, experiencing what Hellstrom calls the entrapped self, imprisoned by immobility, loneliness and difficulty communicating the experience of pain (Hellstrom, 2001). Participants in Hellstrom's study also described themselves in terms of definitions given to them by others, such as a hypochondriac, work-shy, a whiner. Finally, individuals described attempts to maintain consistency with the past self by affirming their previous competence and health, and 
grieving for losses of aspects of self that cannot be continued (Hellstrom, 2001). This process results in what Hellstrom calls "a viscous long-lasting now", or the reduced ability to consider or plan for the future.

Schemas are patterns of associated cognitive representations and also form patterns of beliefs about the self-concept. Pincus and Morley (2001) found that pain self-enmeshment contributes to both cognitive and information processing biases seen in individuals with chronic pain. Such processing biases occur as a result of repeated or ongoing pain that activates a number of schemas. Pincus and Morley propose that three schemas can become enmeshed or activated together when individuals experience prolonged or repeated pain: (1) the pain schema incorporates the sensory-intensity, spatial and temporal features of pain including the emotional state of unpleasantness (p. 607); (2) the illness schema incorporates concepts of identity, duration, causes, consequences and control of a particular illness (that is, the expectations about an illness), and (3) the self-schema, or representation of "who I am and what I can expect and want from life" (Pincus \& Morley, 2001).

Self-discrepancy theorists consider that any discrepancy between the actual self and a future self (hoped-for, feared or ought) generates emotions that in turn influence actions that enable goal achievement towards the hoped-for self. Some individuals with chronic pain, as Hellstrom (2001) found, have difficulty conceptualising a future different from the present. This means there is no discrepancy between the actual self and future selves (because a future self cannot be imagined) thus there is little or no motivation to change (Pincus \& Morley, 2001).

To support this theory, a study by Morley et al. (2005) found that enmeshment between the self-schema and pain explained depression. Enmeshment is more commonly known as hopelessness - that is, the belief that the future is likely to be negative and that there is little chance to influence the situation. Like Hellstrom (2001), Morley et al. (2005) views 
hopelessness as resulting in difficulty planning for or imagining the future. The findings from Morley's earlier study were confirmed by Sutherland and Morley (2008) again showing that self-pain enmeshment influences depression and conversely acceptance. The stronger an individual's self-pain enmeshment, the more he or she finds it difficult to conceive of a future in which positive aspects of self (the ideal self) can be achieved while pain persists. The weaker the pain-self enmeshment, the more readily an individual can imagine him or herself as a being able to achieve the ideal self despite the presence of pain (Sutherland \& Morley, 2008). Sutherland and Morley (2008) consider that the hopedfor self corresponds to the values concept described in Acceptance and Commitment Therapy (ACT), an approach to which I will return to later in this chapter. Kindermans et al. (2010) analysed responses to Hardin's Selves Questionnaire (Hardin \& Leong, 2005) in which participants with chronic pain identified the attributes of their ideal, ought and feared selves. Participants identified characteristics that fell mostly into the interpersonal domain, reflecting the importance of the social role to individuals: that is, the person they want to, feel compelled to, or fear to be within the social environment (Kindermans et al., 2010, p. 652). The second largest group of attributes were those relating to psychological, emotional and physical wellbeing. The authors concluded that, for people with low back pain, the challenges faced in social role functioning in terms of their sense of self are as influential as their sense of self in relation to their health condition. They considered that self-discrepancy theory explained the different emotional responses to chronic pain (Kindermans et al., 2010).

Engaging in occupations is one way individuals express and shape their sense of self (Christiansen, 1999; Harris et al., 2003; Huot \& Rudman, 2010; Phelan \& Kinsella, 2009; Reynolds, 2003; Shank \& Cutchin, 2010). Individuals with chronic pain experience constraints on their ability to engage in preferred occupations (Miles et al., 2005; Osborne, Jensen, Ehde, Hanley, \& Kraft, 2007; Thomas, Peat, Harris, Wilkie, \& Croft, 2004). Chronic 
pain interferes with how an individual can do what is usual and habitual, and it is through becoming aware of disability or functional limitations (rather than pain intensity) that individuals begin to search for treatment (Ferreira et al., 2010a). Participating in daily occupations highlights differences between the past self and the actual self. Differences are recognised because individuals monitor their self-identity in terms of how effectively they carry out everyday tasks, particularly habitual tasks (Charmaz, 2002; Charmaz, 2006c; Magnus, 2001). Goossens et al. (2010) suggested that self-discrepancies only give rise to negative emotions when people are inflexible to the consequences of the pain problem, implying that individuals use an accommodative coping style to manage negative emotions. In other words, that people become positive despite their pain when they disengage from blocked goals and unhelpful commitments, then reorient towards more achievable standards (Goossens et al., 2010).

My findings support and extend the self-discrepancy explanations for adapting to chronic pain. While participants in my study, like those in Hellstrom's (2001), described losing their future focus while making sense, unlike Oliver (1996), when I interviewed them they did not strongly identify with being disabled, or a "pain patient". This occurred after deciding and when flexibly persisting (Hellstrom, 2001; Hellstrom \& Carlsson, 1996). Participants began future planning once flexibly persisting, an indication that they now recognised the world and themselves as coherent. Participants also did not experience the "devastation" identified by Salick and Auerbach (2006) when obtaining diagnostic clarity. Instead, making sense provided them with a sense of relief, allowing participants to begin deciding. Individuals in my study did not appear to experience self-pain enmeshment (Morley et al., 2005; Pincus \& Morley, 2001; Sutherland \& Morley, 2008) once they had decided and began flexibly persisting. Rather, they developed a new self-identity in which pain was integrated and formed a small part. Prior to this, however, self-pain enmeshment was 
somewhat apparent, although participants maintained aspects of self-pain separation and continued with some of their usual occupations.

Similar to the findings of Kindermans et al. (2010), participants in my study acknowledged the influence of social expectations on their participation in occupations. Work (paid and unpaid employment) and parenting were prioritised over discretionary occupations while making sense, but once individuals had decided and were flexibly persisting, occupations reflected a greater embodiment of ideal-self occupational engagement. Finally, as described by Charmaz (2002); Charmaz (2006c); Magnus (2001) participants used occupational existing to discover the effects of pain, while later demonstrated the effect of the reduced threat value of pain on self as they began occupational engaging. Participants engaged in occupations that expressed self-identity and were able to choose new and varied pursuits as compared with when they were occupational existing. Occupational engaging, coping and future planning influence and are influenced by a self-concept that integrates pain as a small aspect of self rather than a dominant illness schema. This aspect of my findings extends the understanding of how and particularly when a new selfconcept integrates aspects of ought, feared and ideal selves as individuals move from making sense to flexibly persisting.

To summarise this section, aspects of self, self-concept and identity emerge and continue to develop over the course of a lifetime. Much of the self is enacted within habitual ways of responding within the world, particularly within the habits of daily life occupations. III health changes the way the body functions and how individuals experience the body, in turn altering the self-concept by creating discrepancies between what is usual (habitual) and what can be achieved now. In response to these changes, social expectations also change and contribute to challenges to the self-concept. When a person can carry through at least some aspects of the self (primarily cognitive representations of "what it means to 
be me") via habits of daily life over time, new self-concepts can also emerge, be integrated with existing representations and thus maintain a sense of continuity.

From my study, individuals who cope well appear to renegotiate their self-identity successfully. They do this by retaining aspects of their past self, particularly values, roles and occupations despite needing to make changes to how these self-concepts are enacted.

Individuals used the process of making sense and the motivation and importance of occupational engaging to decouple schemas of pain, illness and self (Pincus \& Morley, 2001). This occurs at the point of deciding, but continues thereafter.

One factor distinguishing those who cope well with those do not is the degree to which aspects of the past self are retained. When the new self-concept retains familiar aspects of self, and when illness-schema is no longer activated, individuals now identify themselves as "living well". They are re-occupying self. Their sense of self-identity is retained because they are enacting values associated with important occupations. Flexibly persisting prioritises occupational engagement over pain relief, thus maintaining positive emotions despite the substantial modifications to the ways occupations can be carried out. The new self-concept and identity integrates the changes imposed by pain, and once this has consolidated, future planning based on the new self-concept returns. While participants in my study modified their goals as Goossens and colleagues (2010) found, in addition, they modified their self-expectations and their approach to occupational engaging. People who are flexibly persisting look beyond the superficial features of occupation and the strategies they use, and instead consider the functional and contextual outcomes they wish to achieve. This ensures their sense of self is retained, achieving self-coherence, or as Antonovsky (1996) puts it, they "foster repeated life experiences which, to put it at its simplest, helped one to see the world as 'making sense', cognitively, instrumentally and emotionally" (p. 15). 


\section{Coping with chronic illness and disease}

The next two models are theoretical explanations for the ways individuals cope with chronic illness and disease. There is a large body of research supporting both of these models, including some exploring the ways individuals cope with chronic pain. I will briefly outline each model then discuss the findings from my study.

\section{Common Sense Model}

The Common Sense Model of Illness Representations is a social cognitive model developed to explain how cognitive factors influence the ways individuals deal with their health problems. Leventhal (1970) originally developed the model to explain adherence to medical regimens, then, in collaboration with Diefenbach, extended to explain health behaviour more broadly (Diefenbach \& Leventhal, 1996; Leventhal et al., 1992). The model incorporates a self-regulatory approach (that is, it incorporates a feedback loop from the results of actions to appraisals) to show that health behaviours emerge from cognitive appraisals of the threat to health. The model proposes that individuals develop mental representations of their illness based on the information available to them from sociocultural sources, significant others (including health professionals) and his or her own experience of their symptoms (Diefenbach \& Leventhal, 1996. Illness representations incorporate personal experiences of symptoms, and attributions made to a particular diagnosis (Broadbent, 2010). Depending on the attributions, individuals experience an emotional response and thus form actions (Hagger \& Orbell, 2003).

The model consists of five core components or illness perceptions that constitute cognitive representations of illnesses: identity - the name of the illness; timeline - how long it should continue; cause - what caused the illness; control - how well the illness can be controlled, and consequences - the effects of the illness on life domains (Broadbent, 2010, p. 257). Individuals not only hold illness representations, they also have treatment 
representations, or beliefs about the need for treatment, the type of treatment, how long it will take to work and the effects it should have on symptoms. IIIness and treatment representations integrate with beliefs about personal susceptibility to illnesses and ultimately influence the actions individuals take.

Substantial research across a range of health conditions including myocardial infarction, irritable bowel syndrome, diabetes, rheumatoid arthritis, and chronic pain Hagger and Orbell (2003) provide support for hypotheses derived from the model, and in particular between illness cognitions, coping and health outcomes. 


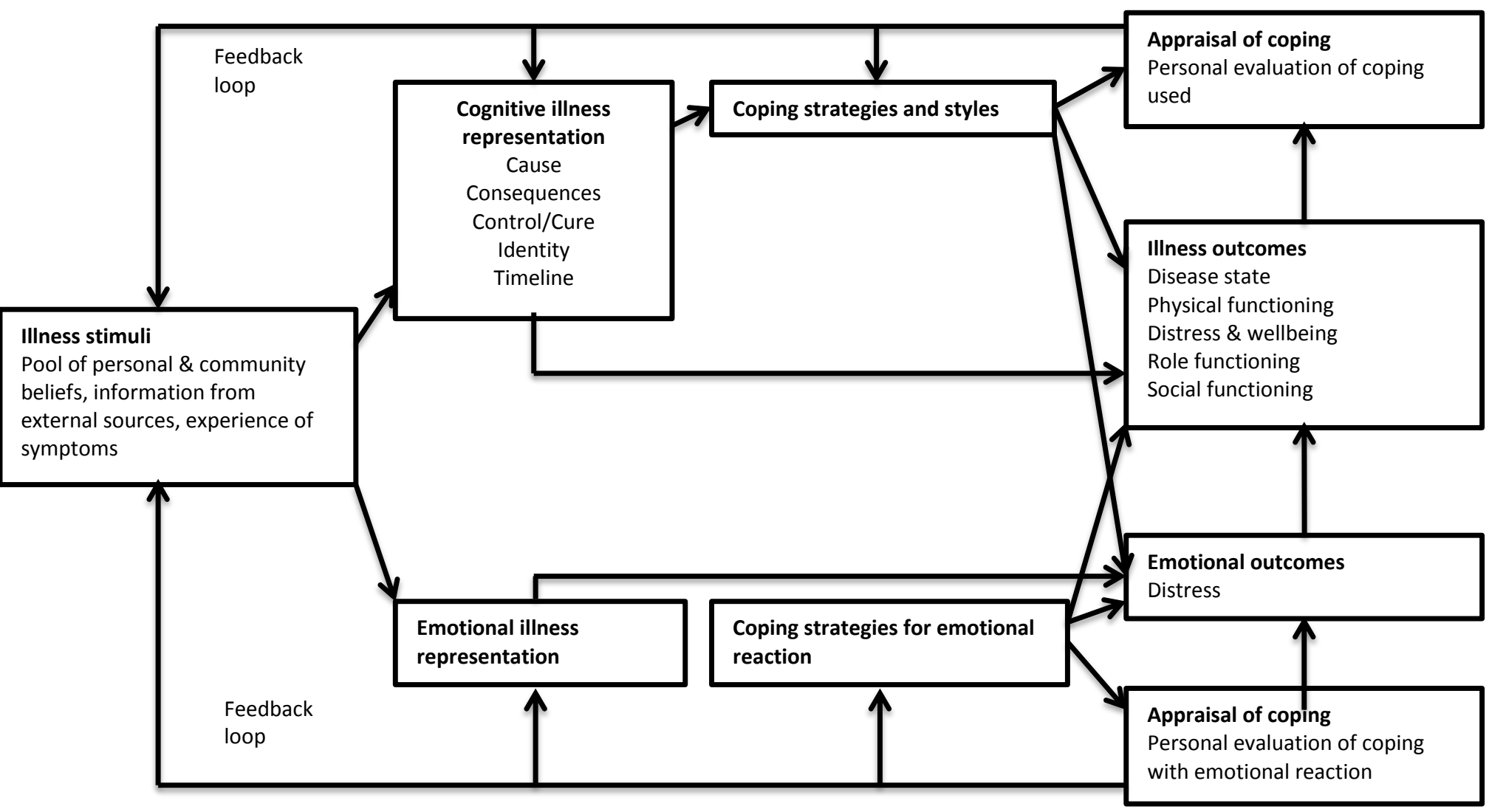

Figure 28. Common Sense Model (Leventhal, Diefenbach \& Leventhal, 1992)

There are similarities between parts of this model and my findings, particularly in terms of the initial phases. People with chronic pain engage in the process of making sense in which they use the knowledge they hold about pain, combined with information from both the community and health professionals to understand what their pain experience means in the context of their life. This process is similar to the first three components of the Common Sense Model: illness stimuli, cognitive illness representation and emotional illness representations, although my findings incorporate a broader consideration of the meaning of this information in terms of the self, self-concept and self-identity. In other words, individuals who live well with chronic pain use making sense not only to understand pain as a symptom or disease, but also in terms of the implications for their life trajectory and identity. Importantly individuals experience the time spent making sense as a period of stasis which disrupts plans for the future. The Common Sense Model does 
not incorporate contextual aspects such as identity or occupation but considers illness independent of life context.

The Common Sense Model fails to explain why an individual might decide to develop new coping strategies, beyond appealing to either human desire to return to homeostasis or the indirect feedback loop from illness outcomes, through coping appraisal to coping strategies and styles while my findings integrate occupation, as an enactment of selfidentity, as the driving force for an individual to cope well.

\section{Moos and Holahan's biopsychosocial framework}

Moos and Holahan developed a framework for conceptualising coping skills and incorporating these into a comprehensive predictive model (Moos \& Holahan, 2003; 2007, p. 109). The framework consists of five sets of factors that interact with one another and influence the coping strategies chosen by the individual. Like the Common Sense Model, it is a self-regulatory model in which feedback from health-related outcomes subsequently influences factors known to influence cognitive appraisal. The five factors, or panels, are (I) Personal resources; (II) Health-related factors; (III) Social and physical context; (IV) Cognitive appraisal; (V) Adaptive tasks; (VI) Coping skills and (VII) Health-related outcomes. 


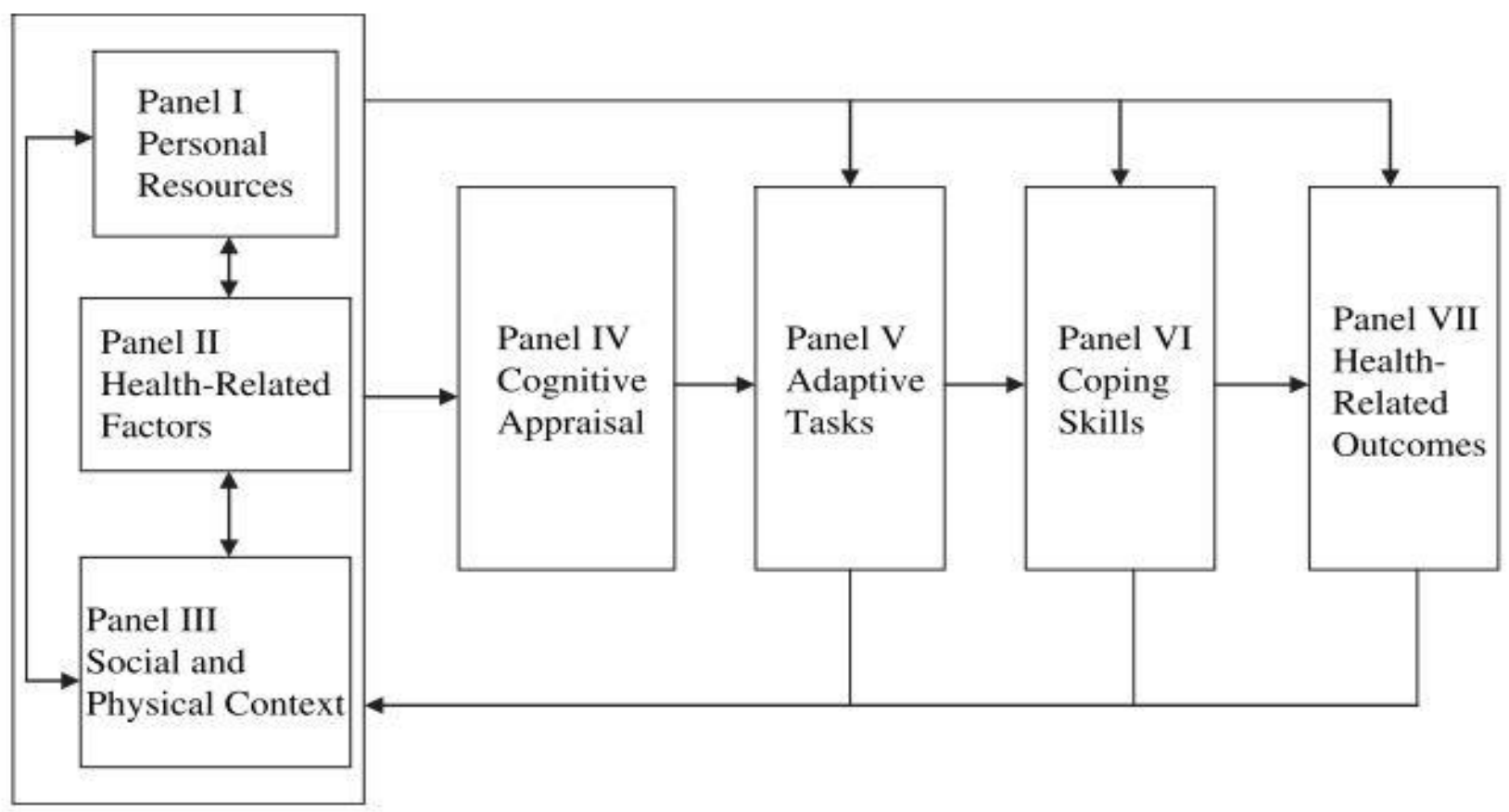

Figure 29. A biopsychosocial integrating framework for coping. Moos \& Holahan (2007).

Numerous factors are identified within each panel such as locus of control, sense of coherence and self-efficacy in Panel I, Personal resources; and relationship quality, relationship responses (punitive or solicitous), and physical features of the environment are associated with Panel III, Social and physical context (Moos \& Holahan, 2007). Panel V, Adaptive tasks, consists of seven groups of tasks, three of which relate to dealing with the specific health condition, while the remaining four apply both to health concerns, as well as general life situations. Moos and Holahan (2007) indicate that the relative importance of each of the seven tasks depends on the individual, their health problem and their unique circumstances. The task groups are: (1) managing symptoms; (2) managing treatment; (3) forming relationships with health-care providers; (4) managing emotions; (5) maintaining a positive self-image; (6) relating to family members and friends, and (7) preparing for an uncertain future (Moos \& Holahan, 2007, p. 112). 
As with the Common Sense Model, coping skills mediate between resources and demands; and consequent health outcomes. Moos and Holahan (2007) identify eight categories of coping skills, consisting of (1) logical analysis and searching for meaning; (2) positive reappraisal; (3) seeking guidance and support; (4) taking problem-solving action; (5) cognitive avoidance or denial; (6) acceptance and resignation; (7) seeking alternative rewards, and (8) emotional discharge (p. 115-117).

These skills may be employed at various times throughout the illness trajectory and be more or less effective, depending on personal and contextual factors. Examples include temporarily denying a diagnosis in the early stages of an illness as a way to cope with overwhelming distress; this approach may provide short-term benefit, but longer-term outcomes are more uncertain (Moos \& Holahan, 2007). A less positive example is attempting to exert control, especially in uncertain circumstances where these efforts may not change the course of the illness (Moos \& Holahan, 2007, p. 117). This style of coping can lead to feelings of guilt or anger, and eventually to demoralisation (McCracken, Vowles, et al., 2007).

Like the Common Sense Model, Moos and Holahan's model explains a good deal about how individuals cope with the emotional and cognitive demands of illness (Moos \& Holahan, 2007). Their model focuses primarily on coping with ill health and the effect of coping on health outcomes, rather than broader concepts such as maintaining or expressing self-identity through occupations (with the exception of those needed for managing health), life roles, or self-expression. Moos and Holahan's model, unlike the Common Sense Model, explicitly considers contextual aspects such as the physical and social environment, taking these into account when conceptualising the resources (and constraints) available to an individual when facing illness or disability (Moos \& Holahan, 2003). The model also incorporates concepts of self and identity as one of the adaptive 
tasks the individual must engage in, that of maintaining a positive self-image (Moos $\&$ Holahan, 2007, p. 115). In their view, individuals use coping skills to achieve adaptive tasks, and thus moderate the effect of ill health on both physical and psychological health (Moos \& Holahan, 2007).

Moos and Holahan believe the focus on coping skills and resources encourages a view of individuals as adaptive and competent despite their health challenges, and they consider the model can guide interventions to promote wellbeing despite illness (Moos \& Holahan, 2007).

In contrast with the theory emerging from my study, while Moos and Holahan's model encompasses more than the Common Sense Model, it does not have a contextual and functional view of coping. Instead, Moos and Holahan divide coping skills into four main groupings on the basis of the individual's orientation in response to a stressor (either approach or avoidance) and the method of coping (either cognitive or behavioural). Thus cognitive approach coping involves analysing, searching for meaning and reappraising the situation; behavioural approach coping includes asking for help and guidance, and taking action to achieve certain goals; cognitive avoidance coping consists of denying the problem, or resigning oneself to the situation, while behavioural avoidance coping includes distraction and emotional expression (Moos \& Holahan, 2007, p. 114). They consider that approach-coping is a more adaptive way of dealing with chronic ill health, and that avoidance-coping strategies are less helpful (p. 117). This is at odds with the findings of my study in which participants were less consistent in their adoption of either approach or avoidance coping, and instead used many different ways of coping while flexibly persisting, using different strategies depending on what they were trying to achieve in a given situation. 
Participants in my study, while acknowledging the demands of their health condition (for example, taking medication, attending appointments, having blood tests), considered health outcomes as part of broader life outcomes. This may reflect their relatively stable phase in the process of living with chronic pain where the underlying disease process is acknowledged as chronic and unlikely to change, or it could be that living with chronic pain involves a different process. Pain is an invasive experience, and individuals identify that almost every facet of life can be affected by it. Thus everything individuals do to manage their pain can influence both health outcomes and general life outcomes. Additionally, participants in my study accepted their pain was chronic, and did not represent an on-going threat in the way that acute pain does. This may mean pain intensity is less relevant to the overall quality of life for people with chronic pain than symptoms of other health problems.

Both Moos and Holahan's (2007) model and the Common Sense Model (Leventhal et al., 1992) have been applied to a wide range of health problems and general stressors. In contrast, my findings form a substantive theory; thus only applies to those living with chronic pain. It is a step less abstract or conceptual than either of these models, having been drawn directly from empirical data, hence is more tied to contextual features such as the diagnosis (conditions where persistent pain is a feature), geographical location (New Zealand), and population (individuals who believe they are living well despite their pain). The similarities between my findings and aspects of these models are unsurprising given the wealth of research contributing to and supporting their development.

A unique feature of my theory, however, is that coping is integrated with engaging in normal routines, habits and daily occupations, and with self-concept. This means the reason for coping is the desire to do what is important in life and to express self-identity, while successfully coping means this has occurred in what I have called re-occupying self. 
Occupational engaging forms a critical part of the motivation for coping in my model, because it supports re-occupying self and achieving self-coherence. Individuals who cope well with pain demonstrate they have successfully integrated self-with-pain into the new identity by and because of occupational engaging. Achieving self-coherence by reoccupying self enables them to begin future planning.

\section{Learning to live with chronic pain}

I now turn to examine some of the literature investigating the processes thought to be involved in learning to live with chronic pain. The process of adapting to a diagnosis has not been examined in as much detail as the factors associated with poor coping, but several qualitative studies identify themes relevant to those in my study.

\section{Gullacksen and Lidbeck (2004)}

Gullacksen and Lidbeck (2004) conducted a longitudinal phenomenological study of women with chronic musculoskeletal pain. They found there were three phases of adjustment to the diagnosis, culminating in a final period of long-term coping. The three phases are summarised in the table below:

\begin{tabular}{|l|l|l|l|}
\hline & Stage I & Stage II & Stage III \\
\hline Past & $\begin{array}{l}\text { "When I was feeling } \\
\text { well" }\end{array}$ & $\begin{array}{l}\text { "Picture of the past } \\
\text { fades" }\end{array}$ & $\begin{array}{l}\text { "New store of } \\
\text { experiences" }\end{array}$ \\
\hline Present & $\begin{array}{l}\text { "Feeling ill, struggle to } \\
\text { restore daily life, chaos" }\end{array}$ & $\begin{array}{l}\text { "Distress, understanding } \\
\text { pain, learning new } \\
\text { coping skills, must } \\
\text { manage" }\end{array}$ & $\begin{array}{l}\text { "Competence and } \\
\text { control of self and pain } \\
\text { increases" }\end{array}$ \\
\hline Future & $\begin{array}{l}\text { "Picture of the future still } \\
\text { intact" }\end{array}$ & $\begin{array}{l}\text { "Picture of future fades, } \\
\text { threatening future" }\end{array}$ & $\begin{array}{l}\text { "A new picture of the } \\
\text { future feels natural" }\end{array}$ \\
\hline
\end{tabular}

Table 1. Coping process developed by Gullacksen \& Lidbeck (2004).

The final stage of long-term coping (not illustrated) was identified as "Maintenance and Living with Pain", and found women referring to "before and after the pain"; the "new 
normal life" for the present, and "managing" for the future (p. 147). Gullacksen and Lidbeck (2004) identified concerns amongst this group of individuals similar to those identified in my study: for instance, they found that, over the course of adjustment "the focus eventually and increasingly was moved from the pain and the body to important parts of life such as family life, social fellowship, working life and leisure time. How could these areas of life be influenced?" (p. 149). Thus, in contrast with the focus on health outcomes prioritised within the models developed by Moos and Holahan (2003) and Leventhal et al. (1992), Gullacksen and Lidbeck's (2004) study reflects participant's concerns about the implications of their pain on their life and identity. They found the medical explanation of pain "dispelled the mystery surrounding the pain", suggesting that knowing this made it possible for individuals to understand their situation in the context of their life as a whole (p. 149).

This finding also emerged from my study, although I found diagnostic clarity and symptom understanding were critical for individuals to experience positive emotions when deciding. Gullacksen and Lidbeck's (2004) study articulates the early stages of adjusting to chronic pain, calling this period a "prelude", in which participants transitioned from temporary adjustments accommodating pain to recognising the problem was now chronic (p. 147). They did not, however, indicate the ways these women developed this understanding, nor the influence of occupation in this discovery.

My findings clearly demonstrate that individuals use three sources of information when making sense: diagnostic clarity, symptom understanding and occupational existing. These three combine to help individuals form their own pain model which later helps them understand that their world is more coherent than they thought, enabling them to define the areas in which they want to develop coping and negotiate occupations so they are more able to fulfil values. 
Gullacksen and Lidbeck (2004) found that the third and final stage of living with chronic pain involves adjusting the self-concept, integrating both expectations from others as well as recognising and implementing new attitudes and behaviour patterns. They did not identify the distinct point of deciding found in my model, and the motivational power of occupational engaging is not clearly articulated. This may reflect the characteristics of their participants who were people referred to participate in a tertiary pain management centre, or it may be that the grounded theory methodology is particularly attuned to identifying and explaining the way individuals' resolve their main concern.

Emotional support was identified by Gullacksen and Lidbeck (2004) as a vital part of adjusting to chronic pain from the onset, and this was when professional support was particularly influential. Emotional support was needed until the participants re-established routines (p. 149). In my study, emotional support and social relationships were acknowledged but were not particularly prominent. This could again reflect the different characteristics of the two groups of participants, but could also represent a different focus of the enquiry.

In my study I was especially focusing on how people resolved the main concern of achieving self-coherence, and while interactions with others were valued, participants' indicated that the process was personal and their responsibility rather than developed as a response to interactions with others. At the same time, Gullacksen and Lidbeck's (2004) study identified two aspects of social support I also found - obtaining a definitive diagnosis and explanation from a health professional, and the individual's desire to decide the level of practical help for themselves rather than, as one participant in my study described it, "being wrapped up in cotton wool". By stepping back to allow people to determine their own need for support, and responding when asked, participants in my study indicated that they felt other people were acknowledging their strengths and independence. 
Gullacksen and Lidbeck (2004) argue that their findings support the need for people with chronic pain to be given a clear diagnosis and explanation, and that until they do people will "regard the pain as mysterious" (p. 151). They suggest individuals receive an explanation based on a neurobiological framework rather than necessarily providing a diagnostic label, finding that their participants valued explanation more than a label. My study findings concur with the first part of their recommendation, with participants in my study also describing their pain as "mysterious" rather than frightening. In my study participants were more concerned with matching their own experience of symptoms with those associated with a diagnostic label. Importantly, my findings strongly suggest diagnostic clarity must convey that "hurt does not equal harm". The message may need to be received more than once, from more than one source and in more than one form before individuals accept it. This aspect of pain-related information is not mentioned by Gullacksen and Lidbeck (2004), although it may be implied within a "neurobiological framework".

\section{Howell (1994)}

Similar discoveries regarding the process of coping with chronic pain in people seeking treatment for their pain have been found by other qualitative researchers. A gendered account of the ways women respond to chronic pain was described by Howell (1994), in which four theoretical categories were identified: (1) The pain takes over (the initial phase of searching for a diagnosis, and losing many aspects of life; (2) Filling my life with pain and despair (responding negatively to chronic pain, other people responding negatively to the woman experiencing pain); (3) Filling my life with new hope (grieving for losses making way for a holistic view of pain as part of themselves); and finally (4) Fulfilling my life with pain (focusing on living well despite pain, which they did not want to "give it that much recognition" (Howell, 1994, p.111). 
Howell's research was carried out at a time when operant approaches to reducing pain behaviour were prominent, and Howell takes issue with advice given to nurses to "validate" the pain then "punish pain behaviour" (Howell, 1994). A key finding from her study is the need for women to have "an empathic bond" with healthcare providers, with her participants expressing the strong need for empathic responses to their expressions of distress (p. 119). As in my study, her participants were drawn from non-treatment-seeking women with chronic pain; thus her participants are comparable with mine and different from Gullacksen and Lidbeck's participants (2004). My findings support the need for a collaborative approach between at least one healthcare provider and the individual, although this does not need to be maintained throughout the lifetime. My findings did not indicate any additional need for empathic expression at times of pain exacerbation, and it is not clear why interpersonal responses feature less than in other studies. This aspect of my findings merits further examination.

\section{Asbring (2001)}

Asbring (2001) explicitly studied identity-transformation in relation to women with chronic fatigue and fibromyalgia, drawing on symbolic interactionism, and research from within medical sociology by Charmaz (2002); Corbin and Strauss (1991); Strauss et al. (1984). As in my study, Asbring (2001) also found that the illness, whether fatigue or fibromyalgia, represented biographical disruption, or an interruption in life trajectory which is further experienced as a partial or even complete loss of identity (p. 315). The other person, now burdened with fatigue or pain, is initially not me, but later becomes integrated within the self as the woman becomes more able to accept changes to activities (occupations), relationships with others, and approaches to carrying out daily tasks (p. 316). Additionally, Asbring (2001) found that some women experienced not only losses but also gains including recognising perspectives they had not considered prior to their illness such as 
the opportunity to review what they valued in life, learning to set limits on others, and greater appreciation for "trifling things in the world" (Asbring, 2001, p. 317). Asbring (2001) does not articulate the processes women used to integrate the new aspects of self, although it appears that learning to accept changes was a key factor. My study found that the process of integration begins as individuals are deciding; by flexibly persisting, they can maintain continuity between the past self, the actual self and the future self. As a result I believe my results provide an explanation as to how people manage the process of integrating self, and perhaps the process of acceptance.

\section{Monsivais (2005)}

Monsivais (2005) introduces a slightly different view of learning to live with chronic pain in her concept analysis of self-organisation in chronic pain. Self-organisation is a process within an illness theory developed by Mishel (1999) and represents "integrating continuous uncertainty into one's being so that it is accepted as the norm, with the result that the individual has a new sense of order" (Monsivais, 2005, p. 147). Monsivais (2005) suggests that moving towards integration is influenced by previous experiences in life, interactions with those around the individual including health professionals, physiological status and allowing time for self-reflection. In her analysis, Monsivais (2005) describes the process as involving the antecedents of "chaos and confusion", failing to be believed, and loss of identity.

Receiving a diagnosis, in Monsivais (2005) view, was experienced as a time of confusion rather than the sense of relief articulated by participants in my study. Self-organisation in chronic pain is thought to involve being believed, accessing resources, and taking action (p. 148). Interestingly, and in contrast to her finding above but in agreement with my findings, she describes obtaining a diagnosis as providing validation "that the pain is real", enabling people with pain to begin to understand their problem (p. 148). Resources 
supporting self-organisation included being able to talk to health professionals and others about issues outside primarily the biomedical, such as hopes, personal philosophy, and how the person and his or her social supports view the situation. These resources particularly influenced the view individuals have of their situation and the future, and through this, engaging individuals in their management. By developing a clearer view of their situation, individuals are more able to take action, or self-manage their chronic pain, and move toward "non-pain" aspects of life, which Monsivais (2005) considers to be a sign of positive adjustment (p. 150).

Finally, Monsivais (2005) discusses consequences of self-organisation in which the individual experiences a transformed identity, new insights and activly participates in their health care. The new identity incorporates a sense of meaning and coherence in which the whole person including life roles, emotions and relationships are considered so that the self is "connected to the past, present and future" (Monsivais, 2005, p. 149). She, like Asbring (2001) highlights the finding that individuals may have a new appreciation for aspects of life that previously were overlooked, and reprioritise various areas thus, in her opinion, demonstrating a greater flexibility within the worldview (p. 150). The final aspect of self-organisation is being an active participant in managing healthcare, and feeling in control and able to make choices about their health. She alludes to this stage incorporating the concept of acceptance, but that this is also a dynamic or settled experience rather than a static one.

Monsivais (2005) integrates the findings from many studies examining the processes involved in learning to live well with chronic pain, and several of her conclusions mirror those obtained in my study. In particular, she describes the final stage of self-organisation as illustrating the development of a new identity that integrates aspects of past, present and future selves. My study extends her work by demonstrating the central role of occupation in facilitating this process. She also found that individuals who are self- 
organised may not follow the advice of health providers as part of the individual's way of both maintaining control but also as part of living a life with perhaps different priorities than health providers (p. 150). My study similarly shows that individuals make flexible and constantly changing priorities in terms of the way they allocate energy, use coping strategies and adhere to treatment recommendations, and that this is an ongoing process into the future.

Taken together, these four studies support my contention that coping with chronic pain is a process over time in which an individual's self-identity is transformed to accommodate the effect of pain on values, priorities and function. Being given a diagnosis appears to play an important part of this process, legitimising the otherwise subjective and unobservable experience of pain, and enabling individuals to more readily accept that their lives must, can and do change. This reflects a social dimension within the process of coping that perhaps has not been as apparent in the pain research literature as have biological and psychological dimensions.

These studies also articulate the need for individuals to make sense of their situation before proceeding to integrate their altered health status into their self-identity. Relationships with health providers in particular are identified as an important part of accepting the reality of chronic pain, with mixed emotional responses to obtaining diagnostic clarity. In my study, the influence of diagnostic clarity and treatment provider behaviour was apparent, lending greater weight to the need to examine clinician behaviour as it affects an individual's progress towards living well. Clinicians' readiness to diagnose a pain problem as chronic, and to move away from pain reduction towards pain management and disability reduction is not as visible in the research literature as its importance in disability would suggest.

None of these studies identified the particular contribution that occupation makes to individuals' awareness of their capabilities and limitations through occupational existing. 
These studies also do not identify that occupational engaging is a powerful way to generate motivation for re-engaging with life and contributing to the new self-concept. Asbring (2001) and Monsivais (2005) allude to occupational engagement once individuals accept their pain and move forward with life, but do not consider the way occupational existing provides a means for symptom understanding, and neither do they consider the motivational aspects of occupational engaging. Occupation appears to be a particularly important aspect of the process of learning to live with pain as identified by my study, but has not been articulated before.

\section{Resilience studies}

\section{New Zealand studies}

To date, there has been little theoretical development in the chronic pain literature to explain why some individuals remain resilient despite pain. Two early studies conducted in New Zealand (Large \& Strong, 1997; Strong \& Large, 1995) examined coping in individuals who, similarly to participants in my study, were not actively seeking treatment for their chronic pain. These studies shed some light on how coping is appraised by those coping well with chronic pain, and what they do. When presenting these studies, Strong and Large pointed out that an idiographic research method can provide greater precision when describing individual's behaviour (Strong \& Large, 1995, p. 373), arguing as I have, that pain coping measures fail to capture much of what it means to use various coping strategies in differing contexts.

In the first study Strong and Large (1995), focus groups were used to explore the meaning of coping to those living with chronic pain. A rigorous thematic analysis was conducted. The findings showed that these participants used different strategies at different times, influenced by the type of pain experienced, the time of day, and where the individual was (p. 367). Associated with the selection of different strategies was a strong need to be 
aware of both bodies and the pain and to choose strategies accordingly. Strong and Large (1995) called this a "somatic focus", and gave examples of participants saying "learning to listen to my body", "my pain tells me different things about what's appropriate to do with it just now" (Strong \& Large, 1995, p. 376). The strategies included those not typically endorsed as "active" strategies such as ignoring pain, attending to pain and lying down, but they also endorsed using regular exercise in the same way as participants in my study did.

As in my study, participants in this study by Strong and Large (1995) described changes in the ways they managed with their pain over time. These changes reflected a growing understanding that pain was no longer acute, but was going to persist (p. 377). Strong and Large (1995) also found there was a shift requiring conscious effort towards what they call "a positive outlook" when individuals recognised the need to take responsibility for their lives rather than wait for a health professional to provide a cure (p. 377). After this shift had occurred, participants in this study recognised that some of their activities would increase their pain, but that this was acceptable to them because they made choices and accepted them rather than being surprised or overwhelmed by them (Strong \& Large, 1995). Actively choosing to engage in activities that increase pain is also a feature in my findings, indicating that the rewards from doing so outweigh the difficulties of dealing with pain. Finally, Strong and Large (1995) found that most participants viewed themselves as copers, able to put up with the pain, acknowledge it and get on with life. Critical factors involved in this appraisal were time and recognising that pain was not going to go away (p. 380). Again, these findings are confirmed in my study, but I have extended this by identifying the factors necessary for deciding, and the contribution of occupational drive to this decision. I have also identified the underlying process of re-occupying self as the process by which individuals integrate the presence of pain into their self-concept. 
Strong and Large (1995) point out that the somatic focus endorsed by participants in this study was not, at the time, endorsed within chronic pain management programmes. If somatic focus is reframed to include mindful awareness of pain, the first published mention of mindfulness for chronic pain was in 1982 (Kabat-Zinn, 1982), while a randomised controlled trial of ACT appeared in 2006 (Dahl \& Lundgren, 2006). The way in which mindfulness and ACT manage somatic awareness or pain awareness is to identify then "make room for" pain by being nonjudgemental of it. This is a slightly different strategy from that described by Strong and Large (1995) and the participants in my study. The use of "somatic focus" or making sense does not just mean noticing and continuing on without regard to pain intensity, instead it means identifying whatever the main priority might be at that time and context and making decisions about what to do and how to go about it as a result. Making these decisions implies invoking a sophisticated degree of meta-cognition, applying a flexible set of decision rules based on awareness of priorities, values and prior learning. Developing a greater understanding of how these decisions are made in the flow of daily life is a necessary next step towards understanding how people use coping strategies to achieve what is important to them.

The second study by these authors was a study using personal construct theory and repertory grid (Large \& Strong, 1997). Participants were again selected for their back pain experience, and were not seeking treatment. Repertory grid is used to identify the idiographic set of interconnected meanings that help define an individual's relationships to others, based on personal construct theory which people make sense of their world by forming constructs to predict what others around them will do (Kelly, 1955; Large \& Strong, 1997). The study aimed to understand how people would construe "coping" within their own construct system.

The findings of this study showed eight constructs of coping in this group of individuals: being genuine and emphasising the reality of their pain; coping but not through choice; 
being in control or having gained mastery over the situation; being stoic; being cheerful and positive and keeping pride; accepting rather than suffering; failing to accept physical fragility, working to gain acceptance from others; and hope (Large \& Strong, 1997, p. 247). Interestingly, participants rated the "ideal self" as being pain free, while ideally liking to be coping better. Large and Strong (1997) indicate that amongst this group, being a "coper" was a divided construct: half the group thought copers had little pain but coped, while the other half thought copers had a great deal of pain but coped (p. 250). They also suggest that this group of individuals thought that copers "had to be genuine and sincere, and have a real physical illness", and that coping was something that had to be done, with the alternative being dependency, which was seen as a luxury (p. 250). Large and Strong (1997) concluded that these participants were ambivalent towards coping: while coping was more positive than remaining dependent and unwell, it is not seen as ideal, leading to the conclusion that "the social role developed ... is seen to be closer to the ideal than their construction of coping" (p. 251). Coping is a way to maintain self-esteem and acceptance by others.

To a certain extent, my findings are similar to those obtained in this study. Participants in my study certainly used coping to support their social role, and experienced a sense of mastery when flexibly persisting. The ambivalence described by Large and Strong (1997) was not strongly present, however, and rather than seeing coping as a "necessary evil" participants thought of coping as a pragmatic "whatever works to get on with life" concept, with occupational engaging providing a positive reason to find and use strategies.

\section{Resilience and vulnerability}

A recent theoretical development of resilience in chronic pain has been initiated by Smith and Zautra (2008); Sturgeon and Zautra (2010) and later Yeung et al. (2012). These 
authors have developed a two-factor model of Vulnerability and Resilience Processes which has been supported by preliminary empirical studies.

Smith and Zautra (2008) suggest that individuals have two main tasks when encountering stress and/or chronic illness: (1) ways to reduce the stresses and conflicts that influence negative mood, and (2) ways to enhance personal features and increase social connections and activities that improve and maintain positive mood (Smith \& Zautra, 2008, p. 799). They consider that resilience resources are stable traits and situations that increase the probability an individual will recover or maintain wellbeing despite stress, while resilience mechanisms are those cognitions, emotions and behaviours the individual employs at the time of the stressor that support recovery or wellbeing (Sturgeon \& Zautra, 2010, p. 107). Sturgeon and Zautra (2010) point out that factors associated with resilience are often believed to be the opposites of factors associated with vulnerability. They believe they are separate constructs with unique contributions, and because there has been extensive coverage of risk factors, preferentially address resilience constructs. The model developed by Sturgeon and Zautra (2010) describes pathways to resilience in which resilience resources and vulnerability traits lead to both resilience mechanisms and vulnerability mechanisms that in turn shape the kinds of coping responses an individual selects. These responses lead to resilience outcomes such as recovery (return to homeostasis), sustainability (maintaining engagement in highly valued activities), and growth (such as new learning, coping efficacy, lower emotional reactivity to pain). Dispositional optimism, pain acceptance and purpose in life are resilience resources that enable helpful or workable coping strategies to be adopted.

Purpose in life is a construct referring to the degree to which individuals believe in their life directions, have goals and beliefs that give life meaning, and demonstrate a high level of intention toward pursuing their goals (p. 107). Smith and Zautra (2004) found that having a high purpose in life enhanced recovery from total knee replacement surgery, having a 
direct effect on physical and mental health. Purpose in life has some conceptual similarity to sense of coherence (Antonovsky, 1996), which I have discussed extensively. Individuals who have a purpose in life have goals, a sense of purpose, and believe that life has meaning (Smith \& Zautra, 2004). Purpose in life also has conceptual similarity to occupational engaging as described in my study findings.

Before discussing how occupational engaging works to generate motivation, it is valuable to conduct a brief review of pain acceptance because acceptance is closely aligned to the processes I have detailed in my model, and is an increasingly relevant concept in chronic pain management.

\section{Acceptance}

Acceptance has been the subject of considerable research within the chronic pain field, although it is a term that many individuals with chronic pain reject, preferring to describe it in terms of dealing with or coming to terms with their pain (Lachapelle, Lavoie, \& Boudreau, 2008, p. 203). Some individuals with pain feel that the term acceptance connotes resignation or giving up (Campbell \& Cramb, 2008; Lachapelle et al., 2008) while the definition of acceptance within ACT is: "the active and aware embrace of those private events occasioned by one's history without unnecessary attempts to change their frequency or form, especially when doing so would cause psychological harm" (Hayes et al., 2006, p. 8). McCracken and Vowles (2008) refer to acceptance as "engaging in activity despite pain, but without restrictions by pain, or attempts to avoid or control pain" (p. 216), and of persisting with valued life activities despite pain.

Various studies show that pain acceptance is an important process variable in cognitive behavioural treatments of both "conventional" and "third wave" varieties. Depending on the definition used, acceptance can be seen as similar to resignation (Lenhart $\&$ Ashby, 1996; Machin, 1998), but the more frequently-used definition by McCracken and Vowles 
(2008) has a neutral or positive tone and is associated with lower reports of pain intensity, less pain-related anxiety and avoidance, less depression, less disability (both physical and psychosocial), more activity and improved work status (Bailey, Carleton, Vlaeyen, 8 Asmundson, 2010; Baranoff, Hanrahan, Kapur, \& Connor, 2013; Branstetter-Rost, Cushing, \& Douleh, 2009; McCracken, 1998, 2010; McCracken, Spertus, Janeck, Sinclair, \& Wetzel, 1999; McCracken \& Velleman, 2010; McCracken \& Vowles, 2007; Thompson \& McCracken, 2011; Vowles, McCracken, \& O'Brien, 2011; Vowles \& Thompson, 2011; Wicksell, Ahlqvist, Bring, Melin, \& Olsson, 2008).

To understand acceptance from an individual's perspective, Risdon, Eccleston, Crombez, and McCracken (2003) in a qualitative analysis using Q-methodology sought the opinions of people from the general public. They found that to this diverse group of individuals acceptance meant (1) acknowledging that a cure of pain was unlikely, (2) a shift away from focusing on pain towards other aspects of life, (3) resisting suggestions that pain is a sign of personal weakness. These findings are comparable to those of McCracken et al. (1999). Risdon and colleagues identified that the critical issue in accepting pain is how pain affects an individual's self-concept (p. 379). Threaded through their eight versions of how people make sense of the idea of accepting chronic pain is the idea that pain does not define the person, but is instead a part of who they are, along with recognising that goals, the individual and how they go about life must adapt. Accepting chronic pain means chronic pain no longer overpowers other parts of life, but individuals need to work on developing the focus towards more rewarding aspects of life (p. 383). Risdon et al. (2003) points out that accepting pain does not appear to involve considering sensory or experiential aspects of pain, but instead is mainly concerned with social role function, and self and social value (p. 383). They conclude that accepting chronic pain means negotiating the process of becoming a different person, and point to work by Pincus and Morley (2001) identifying the need to separate the pain schema from self-schema (p. 384) 
but being unable to describe how this occur, while the final question they leave with readers is whether successfully accepting chronic pain inevitably means accepting a fundamentally changed self-identity (Risdon et al., 2003).

In a review of psychological factors associated with positively adjusting to chronic pain, Keefe et al. (2004) concluded that self-efficacy, or confidence to manage pain, pain coping strategies, readiness to change and acceptance are all associated with reduced disability and distress associated with chronic pain. They point out that using the term acceptance is not especially helpful for people with chronic pain who may interpret this as meaning resignation, and neither should it be used in relation to pain that can be reduced (p. 202). The authors also caution against assuming that acceptance is a stage process, preferring to describe it as a "dynamic, moment-to-moment process" (Keefe et al., 2004, p. 202). Attitudes towards pain have been shown to change with negative affect reduced, when individuals acknowledge pain, give up controlling it, begin behaving "as if" the pain does not inevitably mean disability, and commit to living a satisfying life despite pain (Kranz, Bollinger, \& Nilges, 2010). Kranz et al. (2010) suggest that individuals who can readily disengage from unachievable goals and shift towards setting and achieving goals that can be achieved (accommodative flexibility) are most likely to maintain positive affect and be more accepting of their pain. They consider that this is a source of resilience, and that acceptance as described by McCracken, Vowles, et al. (2007) is an "accommodative way of coping" (p. 1022).

Accommodation involves actively changing the situation and/or behaviour so that the individual can achieve personal goals (Brandtstädter, 2009). Assimilation involves adjusting goals to fit contextual constraints. My study found that people who cope well with pain often changed their goals and adjusted the ways they went about engaging in important occupations depending on their current resources. The same individuals, 
however, often remained committed to their existing goals and self-evaluative standards, commonly associated with assimilative coping; thus it is difficult to categorise the processes neatly into either assimilative or accommodative categories. At the same time, participants clearly demonstrated willingly "engaging in activity despite pain, but without restrictions by pain, or attempts to avoid or control pain" in order to persist in valued occupations (McCracken \& Vowles, 2008, p. 216).

Considerable research has found that greater levels of acceptance are associated with lower levels of distress, depression, disability and even less use of pain relief (Baranoff et al., 2013; Buhrman et al., 2013; Henwood, Ellis, Logan, Dubouloz, \& D'Eon, 2012; Huggins, Bonn-Miller, Oser, Sorrell, \& Trafton, 2012; Kratz, Hirsh, Ehde, \& Jensen, 2013; Lauwerier et al., 2012; Mo'tamedi, Rezaiemaram, \& Tavallaie, 2012; Thompson \& McCracken, 2011; Vowles et al., 2011; Vowles \& Thompson, 2011; Weiss et al., 2013). A recent systematic review of acceptance-based interventions found no difference between traditional cognitive behavioural therapy and acceptance-based approaches, but the authors concluded that it provides a good alternative (Veehof et al., 2011). Similarly, a recent systematic review of mindfulness, one of the common components of acceptance-based interventions, found that results were promising although small study sizes and other methodological problems limit the conclusions that can be drawn (Chiesa \& Serretti, 2011). Including a values element into treatment has been shown to increase tolerance to pain in both experimental (Branstetter-Rost et al., 2009) and clinical settings with the response maintained for up to three years (Vowles \& McCracken, 2008; Vowles et al., 2011)

Despite the considerable work that has been undertaken into pain acceptance, the mechanisms and processes involved in developing acceptance are yet to be fully determined. Using the definition of acceptance by McCracken and Vowles (2008), acceptance indicators would seem to be willingness to experience pain without 
simultaneously being distressed by it, and willingness to engage in activities despite pain. These are the dimensions of the Chronic Pain Acceptance Questionnaire (McCracken et al., 2004). Mindfulness as a practice is increasingly being used as a means to develop chronic pain tolerance (McCracken \& Vowles, 2014; Reiner, Tibi, \& Lipsitz, 2013; Zeidan et al., 2011).

There are situations in which people choose to go through pain - examples discussed in Chapter Two, for example, such as childbirth without analgesia and marathon training. People are willing to endure pain when the outcome is more important than the pain that is experienced during goal pursuit, which now raises the question of how and why occupations support this kind of attitude. Before addressing this, however, it is important to review how occupational engagement helps individuals express themselves and develop their self-concept.

\section{Occupation and meaning}

Making meaning of the world, and events that occur has been the object of study in philosophy, religion, psychology and education. Social cognitive theorists have explored not only differences in temperament or personality, but how these vary from moment-tomoment and therefore influence the ways individuals interpret their experiences (Molden 8 Dweck, 2006). Occupational scientists have drawn on Viktor Frankl's $(1959,1962,1984)$ writings, while Rowles (2008) suggests four domains that inform an individual's search for meaning: (1) achieving a sense of worth through occupation; (2) experiencing fulfillment through interpersonal relationship; (3) exercising the ability to choose one's course of action; (4) and, through the process of simply being, developing understanding and acceptance of one's place in the cosmos (Rowles, 2008, p. 127). Ikiugu et al. (2012) state that "For something to be considered meaningful, it must have personal significance, be important to the individual and be based on the individual's perceptions of his or her 
experiences" (p. 290). This suggests that by engaging in important occupations, individuals both express their identity and obtain feedback from other people and by the degree to which the outcome of their action meets their criteria for success.

I found this was the case in my study where individuals reported remaining involved in occupations to express their identity (for example, a rugby player, a nurse, playing music), and to meet obligations (for example, to be an effective parent, a good employee). Participants also modified the tasks and the way the occupations were undertaken to fulfil the underlying values expressed within the occupations.

Reed et al. (2010) consider that being involved in occupation is a central feature of meaning-making in people's lives. Identity, links to the past (tradition), use of time and even the economic value of having engaged in occupation are features of occupation that contribute to how individuals and their family/whanau and community find purpose and a future focus (Reed et al., 2010, p. 146). Alsaker and Josephsson (2010) found that women with rheumatic diseases used occupations to demonstrate normalcy, or ordinariness of their lives despite the limitations imposed on them by their disease. The women gave examples of being a mother, a partner, a friend of others with the same disease, and the occupations they engaged in and modified to suit their needs in many complex ways provided this sense of ordinariness (p. 66).

Other qualitative studies show how occupation, which by definition is purposeful and has meaning to the individual engaged in it (Christiansen, 1999; Fisher, 2013; Kuo, 2011), provides purpose or motivation for coping with or adapting to health challenges. Kelly, Lamont, and Brunero (2010) compare participating in occupation and the mental health recovery model, finding that individuals who participate in GROW (an international mental health movement in which members run groups to encourage healthy thinking, behaviour and relationships) consider that their recovery from mental ill health involved re-engaging in occupations, where occupations both facilitated recovery and were also a measure of 
recovery. By engaging in normal everyday activities that had previously been abandoned because of mental ill health, individuals developed a sense of purpose, competence, productivity, routine and skills (Kelly et al., 2010, p. 133).

Similarly, Smith, Ludwig, Andersen, and Copolillo (2009) found that women adapting to low vision experienced fulfillment as a part of engaging in occupations, and that doing so enabled them to integrate their losses and abilities. They used alternative methods to achieve occupations, or obtained help; at the same time, they abandoned those occupations they believed they could no longer carry out. By remaining involved in occupations they could still do, the women were able to progress towards accepting that there were some occupations they could no longer do (Smith, Ludwig, et al., 2009, p. 127). Vik, Nygard, Borwell and Josephsson (2008) suggest that participating in occupations has positive effects in part because it enables individuals to "continue to be an agent in daily life" (p. 265) while also experiencing the bidirectional nature of "life itself [as] the agent" (Vik, Nygard, Borell, \& Josephsson, 2008, p. 265). These authors found that three principal aspects of participation have appeared in the rehabilitation literature, mainly directed to research addressing the aging process: (1) studies using the ICF definition and examining social participation; (2) participation as engagement, or how individuals direct their efforts towards participating and engaging in daily life rather than focusing on their ill health, and (3) participation as part of being autonomous, in control of one's own destiny and daily life decisions (Vik et al., 2008, p. 263). Engaging in occupations, therefore, enables social participation, engaging in life and remaining autonomous.

Turning to studies examining occupation and chronic pain, numerous studies have shown that chronic pain can disrupt participation in functional and social occupations (Alsaker \& Josephsson, 2010; Arnold et al., 2008; Borell et al., 2006; Fisher et al., 2007; Harris et al., 2003; Walker, Sofaer, \& Holloway, 2006) and that this is one of the major reasons individuals seek care (Ferreira et al., 2010a; Mortimer et al., 2003). Andersson (2009) 
argues that not only does reduced functioning impact upon quality of life, but it also contributes to increased mortality because individuals do less, have poorer sleep, and engage in risky health behaviours such as tobacco use (Andersson, 2009, p. 1986). Arnold et al. (2008) found that women with fibromyalgia reported difficulty engaging in activities of daily living, leisure activities and paid employment along with social isolation and disrupted relationships due to their health problem. Walker et al. (2006) describe "a catalogue of socio-economic and other material losses including loss of physical and mental abilities, occupational and social activities, job or role" (p. 199) and note that these losses ultimately led to loss of self-worth, future and hope. Participation in paid employment has been the focus of considerable research given its economic significance. Numerous papers describe the impact of pain on both productivity and costs to the organisation and healthcare system (Baldwin \& Butler, 2006; Corbiere, Sullivan, Stanish, \& Adams, 2007; MacKenzie et al., 2006; Marhold, Linton, \& Melin, 2002).

Leisure occupations are thought to help people adjust to and cope with challenges in life by offering diversion, producing optimism, providing an outlet for self-expression, and by giving a context for developing new ways of viewing their situation (Kleiber, Reel, \& Hutchinson, 2008). There is scant information on the impact of pain on leisure or recreation, although Arnold et al. (2008), Harris et al. (2003) and (Ashby, Fitzgerald, \& Raine, 2012) found that people with chronic pain experienced restrictions on the form and frequency of leisure activities, while Arnold et al. (2008) noted that measures of function rarely capture the effort required to engage in, or complete an occupation which may lead to abandoning discretionary occupations such as leisure activities (p. 119).

Another form of occupation in which individuals express their sense of self is in personal care such as preferences in meals that are prepared and eaten, choices in personal hygiene (showering, grooming), the process of selecting and wearing clothing and footwear, and looking after physical and mental health. By successfully engaging in these 
occupations, individuals express their sense of self to the social world, and as Lachapelle et al. (2008) found, help individuals retain their identity and promote acceptance by redefining "normal" (p. 205).

There are substantial grounds, drawing from these studies, for considering occupation as an essential part of self-identity and meaning-making. It is through participating in occupations that individuals express who they are, and receive feedback about their agency in the world. The ability to engage in daily routines and habits can be markedly altered in the face of chronic pain, leading to disrupted self-identity and loss of meaning in life, yet by successfully using occupational drive, those who cope well with chronic pain retain aspects of their self-identity, and continue to express and pursue meaning in life.

\section{Occupation as motivation}

Sturgeon and Zautra (2010) consider that recovery (return to homeostasis), sustainability (uninterrupted engagement in highly valued activities) and growth (coping efficacy, identifying benefits, new learning and less emotional reactivity to pain) are outcomes that indicate resiliency success. From this study, however, I found that engaging in highly valued activities is a source of motivation for developing positive coping responses rather than simply a by-product of coping successfully.

Individuals who live well modify their activities, the way they go about their activities by flexibly persisting so that they achieve what they believe is important at that time and place, rather than maintain their focus on influencing their pain intensity. Occupational existing provides opportunities to recognise and predict the effect of pain and other symptoms on occupation and thus the self-concept. Occupational engaging provides opportunities for individuals to acknowledge the need for new skills, practice using these skills, and via feedback from the results of their actions, establish what is and is not achievable within their current resources. Occupational engaging also provides the means 
through which individuals can experience a sense of purpose and have meaning in life, so that their lives have a direction and a reason to develop coping and persistence. When individuals achieve self-coherence, they seem to have accepted that chronic pain will be present at least in the near future. Although acceptance is an ongoing process revisited by people numerous times (Lachapelle et al., 2008), engaging in valued occupations provides a way to progress the process more quickly.

\section{A functional and contextual perspective}

At the beginning of this chapter, I argued that many views of coping with chronic pain are incomplete because they ignore the nature of coping which must occur both within a context and for one or more purposes. There is always an outcome in mind when an individual chooses a coping strategy, whether it be reduced emotional distress, increased occupational engagement, or to avoid an unpleasant experience. To understand whether a coping strategy works for an individual depends on when, how and why the person is employing that strategy. I provide a brief summary of functional contextualism and the therapeutic application of this philosophy, Acceptance and Commitment Therapy (ACT) to understand the way in which I propose flexibly persisting enables people to live well with chronic pain.

Functional contextualism is a philosophy of science based in pragmatism and contextualism. The philosophy emerged in the mid-1990s during discussions about psychopathology classification when the biomedical model of disease taxonomy was thought inadequate for psychological and behavioural problems. Hayes and colleagues (Hayes, Wilson, Gifford, Follette, \& Strosahl, 1996) proposed a functional classification approach in which the signs and symptoms are organised in terms of the functional and contextual processes that are thought to either produce or maintain them (Hayes et al., 1996, p. 1154). 
Two primary concepts important in functional contextualism are: (1) the unit of analysis, which is the whole event (rather than components of an event); and (2) its truth criterion, which is effective action, or workability (Twohig, 2012). In other words behaviour is evaluated in terms of whether it helps the person to do what is valued, or important within the context of his or her own life and values (Hayes et al., 2006), rather than considering it in isolation. Pathology, therefore, is rejected and replaced with the notion of how well an individual is able to meet his or her own criteria for success.

Hayes argues that many of the efforts individuals use to control or manage events or experiences that cannot be controlled or managed contribute to or generate distress (Hayes et al., 2006). The same argument has been applied to the efforts individuals use to control or manage pain (McCracken \& Vowles, 2008).

Much pain management focuses on reducing pain intensity through surgery, pharmacology, procedures, exercise, relaxation, distraction, activity pacing and much more, including cognitive therapy which aims to change thought content about pain and thus reduce distress and pain exacerbation (Day et al., 2012; Main et al., 2008; Turk, 2002; Turk et al., 2011). Effect sizes and numbers needed to treat have, irrespective of the approach used, been modest (Williams et al., 2012; Woolf, 2010). Many individuals continue to experience lifelong chronic pain over which they have little influence (Ohayon \& Stingl, 2012; Raftery et al., 2012; Reitsma, Tranmer, Buchanan, \& VanDenKerkhof, 2012; Smith \& Torrance, 2012). Paradoxically, in order to change thoughts about pain, to relax the body to reduce pain, or to pace activities to avoid exacerbating pain, as taught within cognitive behaviour pain management programmes, the individual must become more aware of his or her thoughts about pain, must often focus attention on pain to complete thought records or activity diaries, and pain can continue to be the ongoing focus of life while 
remaining distressing to the individual (Crombez, Viane, Eccleston, Devulder, \& Goubert, 2013; Goubert, Crombez, Eccleston, \& Devulder, 2004).

Psychological inflexibility, or experiential avoidance is the term used to describe the efforts individuals use to control their experience with unpleasant emotions, events or actions (Costa \& Pinto-Gouveia, 2013; Esteve, Ramirez-Maestre, \& Lopez-Martinez, 2012). When this concept is applied to chronic pain, efforts to reduce or remove pain such as taking medication, avoiding certain movements and occupations, or reluctantly engaging in them with high levels of distress represent experiential avoidance. While these approaches are workable in the context of acute pain, many of these are ineffective with chronic pain and contribute to distress and disability.

When psychological flexibility replaces experiential avoidance, individuals are described as accepting their thoughts and emotions, and taking action in a way that aligns with their values. Participants in my study described the process of engaging in valued occupations as motivating itself, and the outcomes more meaningful than changes to pain intensity. They set about engaging in occupations to maintain and enhance their quality of life, to remain true to their self-concept, and as a result, the qualities and value of occupation were the focus rather than pain. These individuals employed similar approaches to those described in ACT. They used both occupational engaging and coping to enable them to live the life they want to lead.

While participants in my study reject the term acceptance because of the connotations of giving up hope, at the same time they demonstrate many of the characteristics that researchers argue are important markers of psychological flexibility. That is: participants chose to persist with occupations that were important in their lives and that enabled them to maintain a positive self-identity and sense of coherence. While doing so, they were aware of their pain but considered it largely irrelevant in terms of what they choose to do. 
They had developed the capacity to be non-judgementally aware of their pain when it suits their current intentions. Thus, as one participant stated:

"I can feel my pain, I know it's there, and I know it's going to be really bad after I've gone for a walk, and I can even think I don't want to go for a walk, but I just put it to one side because it's worth it just to get out and be active."

At odds with McCracken and Vowle's (2008) definition of acceptance (p. 216) participants in this study did not completely stop searching for ways to reduce their pain. Instead, and similar to other qualitative studies such as Campbell and Cramb (2008); Lachapelle et al. (2008); Sofaer et al. (2005), depending on a balance of costs and benefits, and current and future goals, individuals evaluated treatments offered and chose whether to engage in them, and/or how much to invest in them. As one participant said "I can take something useful from anything I'm offered" and another "I don't want to give up hope of something that might help reduce the pain, but I'm not going to stop my life just on the off-chance."

Flexibly persisting invokes the concept of committing to what is important but not rigidly so. It incorporates the notion of choosing to change goals, use different coping strategies and prioritise different values depending on the context. In my study, participants' contexts changed frequently: individuals employ a range of strategies in the context of prioritising multiple values, thus flexibly persisting involves having an extensive repertoire of strategies to draw on, and willingness to prioritise different aspects of life at various times. Coping, it seems from my study, involves complex, moment-by-moment decisions about what is relevant at that time and in that context. It implies being comfortable with both accommodating goals and assimilating them, being aware of values and attitudes but using them to guide action in a flexible way. This means sometimes an individual might choose to rest, for example, because fatigue or pain are interfering with engaging in valued occupations, but at other times, elect to persist despite exacerbating their pain and fatigue 
because the end justifies doing so. The stance adopted by participants was one of predominantly choosing to control their actions rather than invariably attempting to control pain. The "workability" of flexibly persisting is, therefore, high within the context of each individual's life, although to an onlooker may not always fit with a traditional coping taxonomy. Flexibly persisting is an ongoing approach to acceptance which is, according to McCracken (2005), not a decision or belief about pain but a process by which patients begin to make daily lifestyle choices that maximize their quality of life (p. 168).

\section{Metacognitive skills}

The contextual nature of flexibly persisting is crucial when attempting to understand how people get on with their lives. Functional contextualism means taking into account the underlying intentions of the individual within that context: that is, considering what the person aims to achieve at this time, in this place, within this set of circumstances. As my findings show, this is an on-going process where individuals identify different priorities at various times, finding ways of achieving those priorities by using any available coping resources. Individuals flexibly persisting can evaluate situations quickly to rapidly recognise and interpret interoceptive and exteroceptive stimuli against actions they may need to take to achieve future outcomes. They, therefore, appear to have high metacognitive abilities.

Metacognitions are commonly described as "thinking about thinking", or, as Delahaij, van Dam, Gaillard, and Soeters (2011) put it, metacognition is "an individual characteristic that encompasses insight into one's emotional and physiological reactions and coping behaviours during stressful situations as well as the conscious regulation of these reactions and behaviours" (p. 52). These authors found that, in acute stress situations, metacognitive awareness mediated coping effectiveness through task-focused coping style and coping self-efficacy (Delahaij et al., 2011). In other words, being aware of one's 
own reactions and coping approaches within stressful situations, and being able to regulate these responses directly, influences the level of confidence an individual has in their ability to cope successfully. In turn, this enables achievement by remaining focused on what is needed to complete the task as opposed to focusing on mitigating negative emotions.

In another study of metacognition and stress, Beer and Moneta (2012) obtained findings that also support the positive contribution of positive metacognitive awareness on coping. In this study, they found that confidence to stop unhelpful emotions and perseverative thoughts was negatively correlated with perceived stress and unhelpful coping, while confidence in using own emotions as a guide to action, avoiding immediate reactions, problem solving and confidence in setting flexible goals predicted adaptive coping. They put forward the idea that by viewing thoughts as cues rather than facts, worry and unhelpfully rigid thought processes are circumvented, enabling creative and flexible approaches to be brought into play (Beer \& Moneta, 2012). This is a process very similar to cognitive defusion in ACT. There are also parallels with the positive emotions generated when engaging in occupations that produce flow (Csikszentmihalyi \& LeFevre, 1989; Reid, 2011).

Neither Delahaij et al. (2011) nor Beer and Moneta (2012) conducted in populations with chronic pain, and I was able to identify only one study in which metacognitions and chronic pain are discussed. A study by Yoshida et al. (2012) looked at the relationship between metacognitions and beliefs about pain. The findings support the idea that particular metacognitions, notably reappraisal and distraction, were associated with greater perceived control over pain, while those involving worry and punishment were associated with greater catastrophising. Taken together with the two studies I presented above, the ability to stand back from the immediacy of judgements about the present, 
along with confidence in one's ability to regulate cognitions and emotions, and make effective choices about the best actions to take appears to be an important aspect of coping that has yet to be thoroughly examined.

Individuals reaching flexibly persisting appear to have developed effective metacognitive awareness and are therefore able to distance themselves from the content of their thoughts and emotions. They can regulate their thoughts, and refocus on priorities. They demonstrate confidence in their ability to do what is needed to achieve whatever their priorities are at that time. Understanding metacognition in chronic pain seems to be a fruitful area for more research to understand flexibly persisting.

\section{To summarise}

Within this chapter I have integrated the literature on four main topics that are relevant to my findings. The model is a mid-level, substantive model of living with chronic pain, and selected models of coping are used to inform and situate the theory. When coping with a major stressor such as chronic pain, identity is initially threatened as the symptoms and signs of chronic pain challenge assumptions about what can and cannot be carried out in daily life. The overall process or main concern of individuals who cope well with chronic pain is achieving self-coherence, and by doing so re-occupying self. By successfully engaging in this process, individuals describe themselves as living well, remaining involved in valued occupations, renegotiating an identity that accommodates the effects of chronic pain.

Similarities exist between Leventhal's (1992) Common Sense Model (CSM), and the process of re-occupying self, particularly the initial steps illustrated in the CSM of developing cognitive and emotions illness representations. Individuals draw on a wide range of sources when making sense, similar to the Common Sense Model, but unlike this 
model, the information used by people who cope well with chronic pain is used not only in coping, but also to inform and adjust the self-concept.

Moos and Holahan (2003) integrate several additional aspects within their biopsychosocial model but like Leventhal's (1992) model, their model omits individual values and occupations. They also dichotomise coping into four types rather than considering them within a functional and contextual framework. Within the model of living well with chronic pain in contrast, coping emerges in the context of occupational engagement as occupations inform and express the self-concept. Coping strategies are neither good nor bad, but rather are more or less useful in the context of the time, place and purpose for which they are being used. This means judgements about the utility of any coping strategy must be made in the context of an individual's occupations and values rather than a strategy being deemed helpful or unhelpful in isolation.

The first phase of re-occupying self involves making sense, consisting of three important processes. Diagnostic clarity equates to parts of Leventhal's (1992) model, but extends this model by including processes of symptom understanding and occupational existing used by individuals produce an idiographic model of pain. Diagnostic clarity features in qualitative studies discussed above (Gullacksen \& Lidbeck, 2004; Monsivais, 2005), and fulfils an important social role in validating and reassuring individuals that their problem is recognised and understood. Symptom understanding is also found in qualitative studies such as Strong and Large's (1995) study where "somatic awareness" formed an integral part of the way non-treatment-seeking individuals managed their involvement in occupation. Finally, occupational existing is the process used to predict the effect of pain on occupation. This finding has also been identified in studies by (Charmaz, 2002; Charmaz, 1995, 2006c), confirming its importance in understanding the processes people use to understand the impact of disease on the self and self-concept. 
Occupational engagement enacts personal values, thus individuals both express their identity and generate feedback through the degree to which the outcome of their action meets their criteria for success. Feedback from others in response to individual's occupational engagement also provides information about self-concept. Occupational drive provides the reason for coping, and coping is how individuals remain engaged in occupation. People who cope well with pain retain a strong drive to remain occupationally engaged, in part because it enables them to maintain valued aspects of their self-identity, but also because it enables them to develop new aspects of identity that can be projected into the future.

People who cope well with pain can be said to have learned to accept their pain. While this is a term many people with chronic pain do not endorse (Lachapelle et al., 2008), participants in this study demonstrate high levels of activity engagement with low levels of distress, despite acknowledging moderate levels of pain. Additionally, individuals who cope well with pain demonstrate effective nonjudgemental awareness of their pain: that is, they do not avoid experiencing their pain, but they do not place importance on it nor use it as an exclusive guide to activity. Unlike McCracken and Vowles' (2008) views of acceptance, participants in this study did not stop looking for ways to reduce their pain however they were not wedded to pain reduction as an outcome. Instead, they were flexibly persisting in the context of their life, evaluating the worth of any intervention against other contextual factors.

For an individual to identify as living well with chronic pain, he or she will have progressed through the two phases of the grounded theory of Living Well, adopted Flexible Persistence, and be both self-managing his or her pain, and fully engaged in valued occupations. This model explains how and why people achieve Flexible Persistence as they experience it. 


\title{
Chapter Seven - Contributions, Limitations,
}

\section{Opportunities and Conclusions}

\author{
"Albert grunted. "Do you know what happens to lads who ask too many questions?" \\ Mort thought for a moment. \\ "No," he said eventually, "what?" \\ There was silence. \\ Then Albert straightened up and said, "Damned if I know. Probably they get answers, and \\ serve 'em right." \\ Terry Pratchett. (1987). Mort, Random House, p. 52
}

At the beginning of this research, I posed questions about how some people appear to live well despite experiencing chronic pain. I wanted to understand how they managed their pain, and what could be learned from them so that clinicians might be able to help those who cope less effectively. In grounded theory terms, I wanted to identify the main concern of people living well with chronic pain, and understand how they resolved this.

This chapter will bring together the findings obtained from undertaking the classical grounded theory of living well with chronic pain, material from relevant literature, and issues discussed within this thesis to provide some tentative answers to these questions. The conclusions developed from these findings may provide clinicians with ideas that can be integrated into clinical practice, thus demonstrating that the classical grounded theory fits, works and has relevance in the field of chronic pain self-management. 


\section{Answering the research questions}

The main concern of people living with chronic pain is how to achieve self-coherence. When the world and self-identity lose comprehensibility, manageability and meaningfulness because pain intrudes, it is hard to rely on what has been learned from the past. The self becomes hard to recognise. To reduce the distress associated with discrepancies between the ideal self and the current self, individuals with pain embark on a process of discovery. This process, when successfully completed, allows them to reoccupy self in a way that feels comfortable and in which they feel "at home", enabling individuals to express important values as they look to the future.

Re-occupying self involves two important phases divided by a process of deciding. In the first phase, energy is spent making sense of the implications of chronic pain on the self. Diagnostic clarity, symptom understanding (notably that pain does not necessarily mean damage is occurring), and occupational existing contribute to developing an idiographic representation of pain.

Deciding to turn from patient to person is facilitated or hindered by interactions with clinicians and occupational drive. This process begins once individuals have completed making sense and possess a strong occupational drive. Occupational drive provides motivation to become an active agent in life again, to express important aspects of the self-concept. Clinicians influence this process positively by supporting occupational engaging and by individualising treatments, whereas they can negatively influence deciding if they fail to provide diagnostic clarity, prolong efforts to abolish pain, or fail to indicate that pain does not necessarily mean harm.

The second phase of re-occupying self is the process of flexibly persisting where occupational engaging and coping allow individuals to begin future planning. Individuals 
form a coherent self-concept in which they re-occupy the important or valued aspects of themselves by completing this process.

\section{Contributions to knowledge}

Despite the enormous investments individuals and organisations have made in investigating the problem of chronic pain, there are many aspects that have yet to be understood. This thesis as a whole makes several contributions to knowledge of chronic pain in a population seldom studied: the experience of those who remain resilient and have learned to live well despite ongoing pain.

In Chapter Five, I identified what I believe are the main contributions of this thesis. I consider the process of re-occupying self explains how people move from merely "coping" to instead consider themselves to be "living well" despite continuing to have chronic pain. In essence, this process explains how individuals re-develop a sense of self that expresses important values and provides meaning and continuity to life. Given the characteristics of those who describe themselves as "living well", the process of re-occupying self also gives an understanding of how individuals develop acceptance of their pain. Acceptance is emerging as an essential concept in coping positively with chronic pain. Knowing that individuals can achieve acceptance by making sense and flexibly persisting, and particularly by recognising the influential role of occupation in this process provides researchers and clinicians with some powerful ways to facilitate this transition.

Making sense involves processes that have been recognised in other accounts of coping with illness. Individuals, by obtaining diagnostic clarity, receive social validation that their internal, subjective experience is "real', or at least has recognisable features. Diagnoses enable individuals to justify their behaviour and alter roles in line with social expectations. The diagnostic process is an important contribution made by clinicians because it invokes social roles and responsibilities. A critical feature of diagnostic clarity in my findings is the 
need to convey the permanence or chronicity of chronic pain. Individuals can stop their search for ways to return to "normal" and begin to contemplate the need for re-occupying self by learning that their pain will remain. Understanding that chronic pain is permanent as requirement for learning to accept pain is not a novel finding: it has been identified in many studies cited in this thesis. What is surprising is how little research has been carried out to ascertain the best ways to convey this news, although perhaps less surprising is the reluctance clinicians express towards conveying this information to patients. Again, this is an aspect of clinical practice, particularly within a primary care setting, that merits further attention. There must be a balance between conveying the long-term nature of chronic pain, and contributing to catastrophising, or raising the threat value of pain.

While clinicians also provide information to inform an individual's symptom understanding, this process is a personal and individual one. Symptom understanding is rarely featured in a structured way within chronic pain management programmes (Large \& Strong, 1997; Strong \& Large, 1995), perhaps because of lingering beliefs from the operant conditioning paradigm that individuals will avoid engaging in activities by attending to pain, or risk becoming hypervigilant. While avoidance may be one outcome of attending to pain, somatic awareness or symptom understanding allows individuals to develop an understanding of "normal" or typical levels, qualities and patterns of pain. By doing so, they become less concerned with daily fluctuations which are common in chronically painful disease. Recognising that symptom understanding is an important part of making sense but can also risk hypervigilance and avoidance raises an important research question: how can clinicians effectively guide people to develop symptom understanding? Associated with symptom understanding, individuals also use occupational existing to help them when making sense. At least some of the qualities associated with occupational existing (such as reducing engagement in leisure, focusing on what must be done rather 
than discretionary occupations) occurs because having a future focus depends on being able to make predictions from reliable evaluations of self. When the self-concept is disrupted and capabilities uncertain, it is difficult to make plans. A certain amount of energy is also expended while making sense to attend treatments, deal with the disruption to ordinary life occupations and in obtaining diagnostic clarity and symptom understanding. Consequently, occupational existing maintains functional status quo. More importantly, however, the process provides information on how symptoms affect selfconcept and occupational engagement.

Occupational existing, like symptom understanding is not often featured in chronic pain management programmes. Occupational existing, and the subsequent lack of motivation displayed by those with chronic pain, is seen as a negative element in clinical work (Allegretti et al., 2010; Parry, 2004). By repositioning this lack of future focus as an understandable response to uncertainty, and by using existing occupations to aid in making sense, some of the resistance to therapeutic efforts may be reduced. Moreover, when occupational drive is used to support deciding, or the transition from patient to person, motivation for occupational engagement is harnessed.

Clinicians may be able to develop structured techniques for individuals who are making sense. They may be able to support occupational existing as a method for developing predictive rules on the effect of pain on occupational performance by working with individuals to generate graded hierarchies of occupations that, in effect, become behavioural approach tests (BAT). BAT are used in behavioural pain management, particularly during exposure therapy, to enable individuals to experiment and reality test assumptions about consequences (Vlaeyen, Morley, Linton, Boersma, \& de Jong, 2012b). Deciding has emerged as a pivotal point in the process of re-occupying self. Deciding involves occupational drive but is strongly influenced by clinicians. I have described the 
critical influence of clinicians who may inadvertently delay an individual deciding by maintaining the hope that life can return to "normal". Particular actions carried out by clinicians such as suggesting new investigations, recommending pain reducing procedures, failing to provide diagnostic clarity, and continuing to reinforce pain as a guide or an indication of ongoing tissue damage can all obstruct progressing towards deciding. Acceptance is an important concept in pain management today. Without accepting the presence of pain, individuals are inclined to reject or defend against the experience, and expend effort doing so. Acceptance implies that pain becomes integrated into how life is lived. I contend that integration involves re-occupying self. Flexibly persisting as a process explains how re-occupying occurs. Deciding is the point when individuals weigh the cost of rejecting pain against pursuing a life with pain. It is at this point individuals are beginning to develop a self-identity that retains past aspects of self-concept into the present, incorporates pain schema, and projects capabilities into the future. Pain schema are present: this is evident from the many incidents provided by participants in my study when they describe being aware of pain, but some of the associations between pain and emotions and predictions about capabilities are loosened. Participants described deciding as a precise point in time. My study asked individuals to recall this process and it is now essential to study the process prospectively and to establish ways to present the necessary information in a way to facilitate deciding at an earlier stage.

Deciding can be facilitated by assisting individuals with making sense. By ensuring diagnostic clarity, symptom understanding and occupational existing furnish people with a personalised model of their pain, they may be ready to consider a future with pain as part of their self-concept. Motivation to begin deciding is, however, initiated when individuals identify an occupation or occupations that are (or could become) integral to their selfidentity. Exactly how individuals resonate with certain occupations is not yet clear, but it is 
apparent that social obligations, feedback from others, and self-efficacy as well as interest and pleasure and environmental affordances contribute (Abrahams, 2008; Christiansen, 2000; Christiansen, 1999). My study identified that occupations embodying important values form a strong link between past self-concept and the new self-concept. Values are an integral part of ACT and including values-based action has demonstrated positive outcomes after pain management programmes (Hayes et al., 2006; McCracken \& Vowles, 2008; McCracken \& Yang, 2006; Vowles \& McCracken, 2008; Vowles et al., 2011). Guiding individuals at the point of deciding towards clarifying values and identifying occupations in which values are expressed may contribute to deciding at an earlier stage than if individuals must find these themselves.

Once deciding is complete, flexibly persisting becomes the focus of those who are reoccupying self. Occupational engaging is the process in which individuals begin doing again. Coping emerges as individuals begin occupational engaging. By implication, attempting to develop coping in isolation from occupational engaging is unlikely to be successful. Perhaps an explanation for the relatively small effect sizes from chronic pain management programmes is that coping is not embedded in occupational engaging. Skills developed away from the contexts in which they need to be used may be difficult to retrieve when needed. Occupational engaging may involve individuals using a graded exposure approach to develop coping at the same time. Studies examining this process in daily life contexts would provide insights into whether this is the process used by individuals who do not seek treatment. Clinically, people who seek treatment for their chronic pain may benefit from engaging in this process in a structured way, where learning experiences can be graded to increase self efficacy.

Reconceptualising coping strategies in a functional and contextual way removes the need for clinicians to judge whether groups of strategies are "adaptive" or "maladaptive", but 
does increase the need for clinicians to become confident with the notion of "workability". My research supports the need to consider how well a strategy helps individuals achieve important goals when they use a strategy, and to consider how many ways a person can identify to achieve what is important to them. It is flexibility, choice and options in coping that enable rather than their classification into some nomothetic scheme.

Flexibly persisting, and particularly the functional and contextual nature of coping as identified In my study, aligns well with ACT as a therapeutic approach. ACT encourages psychological flexibility, a concept which, in practical terms, is thought to lead individuals to use a wider range of ways to live full and productive lives (Fish, Hogan, Morrison, Stewart, \& McGuire, 2013; Hayes et al., 2006; McCracken, 2010; McCracken \& GauntlettGilbert, 2011; McCracken \& Morley, 2014). ACT, while no more effective than cognitive behavioural approaches to chronic pain (Williams et al., 2012), aligns readily within many health discipline's philosophies and particularly within occupational therapy's philosophies. The findings from my study suggest occupational therapists may provide a unique contribution to ACT as a clinical treatment for pain by extending the understanding of occupation within individual's lives.

\section{Recommendations for improving chronic pain management}

Drawing from the information obtained in this study, tentative recommendations for helping people who cope less well with chronic pain may be made. These are (1) to provide individuals with a clear understanding of the likelihood their pain will persist; but importantly (2) for individuals to be informed that it is possible to live well despite experiencing moderate levels of pain, provided that (3) individuals are supported to accept that pain is largely irrelevant in terms of its threat value; (4) individuals are helped to identify what is important and valued in their life so they may use this as a positive focus for the future and for developing self-identity with chronic pain integrated as a small 
component; and (5) that individuals become proficient at using nonjudgemental awareness of pain, exercise for mental wellbeing and can use "whatever works" in a flexible way in the context of their daily life.

These recommendations point to several positive actions for a public health approach to mitigating disability associated with chronic pain. Firstly, endeavours similar to those used in the Mental Health Foundations "Like Minds, Like Mine", and the Arthritis New Zealand Ankylosing Spondylitis media campaign, positive role models demonstrating living well with chronic pain could help bring about a change in community expectations for living with chronic pain. Secondly, media campaigns and internet-based approaches to living well with chronic pain similar to those used in the Health Promotion Agency campaign for depression, which use Sir John Kirwan as spokesperson for normalising depression, and promoting exercise and self-care, could provide practical assistance and acknowledgement to those living in the community with chronic pain. Finally, education, training and funding support to organisations in the community who provide occupationfocused groups for individuals who are not currently in paid employment, such as the Men's Sheds movement. This could enable these organisations to extend their reach to individuals who experience chronic pain, encouraging these individuals to participate in occupations with support, enabling them to develop coping strategies as a result of this involvement.

Alongside these approaches, several clinical recommendations may be made:

- As discussed above, treatment approaches integral to Acceptance and Commitment Therapy are supported by this study's findings. People who are naïve to chronic pain management and ultimately live well with their pain develop these skills naturally; therefore ACT would appear to be a good fit. 
- Individuals who cope less effectively with pain may benefit from being helped to identify those aspects of self-identity they can retain despite their chronic pain. This may involve clarifying values and establishing how these are still enacted in daily life, identifying and acknowledging continuing occupations that provide positive feedback about self-identity, and identifying those values, occupations and aspects of self that can be set as therapeutic goals or outcomes for interventions.

- Those people who are unwilling, or not strongly motivated, to engage in learning self-management strategies may benefit from being helped to first return to valued occupations. By doing this, the need for developing effective coping strategies may be generated. At the same time, health professionals may become aware of ways of coping they had not considered as useful or positive, as they observe how individuals find ways of achieving what is important to them in the context of their lives.

- The "small acts" that helped define trustworthy clinicians can be readily implemented, and, from this study, clearly indicated to people with chronic pain that they were being taken seriously, and were acknowledged as individuals. Although it may seem counterintuitive, using technology to support individualised information may be useful, such as sending text messages between appointments, personalising an exercise sheet with the person's details, and emailing test results. Interactive web-based or electronic device-based applications may also help, allowing two-way communication between clinicians and people with pain.

- More controversially, assisting clinicians to gently provide clear prognostic information about chronic pain may be an important step. This is controversial because anecdotally clinicians may consider this to remove hope for the patient; some clinicians believe that chronic pain can always be eliminated if the "source of the nociception is found", particularly those with an interventional procedures- 
based orientation (Roth, Geisser, \& Williams, 2012), and because of the tendency for clinicians and patients to hold very different models of the relevance of biomedical versus psychosocial variables (Allegretti et al., 2010; Crowley-Matoka et al., 2009). Clinician attitudes towards breaking bad news, the time required to do so with appropriate empathy, and knowledge of where to refer a person in this situation are all aspects of clinical practice that warrant being further examined so that practical solutions can be developed. At least part of the difficulty implementing this behaviour change is the need for adequate time to talk with patients, along with ensuring clinicians, particularly in primary care, have somewhere to refer those individuals for adequate and appropriate self-management support.

\section{Limitations of this study}

Every research study has limits on how broadly the findings can be generalised. In this section, I will discuss the limitations of this study particularly relevant to researching pain, as well as limitations that apply specifically to this study. I have already discussed the limitations relevant to classical grounded theory as a methodology in Chapters Three and Five.

\section{Contextual limitations}

Social research is bound, at least to a certain extent, by contextual factors. This study has been conducted in New Zealand, between 2011 and 2013, by a woman in her mid-40s with an occupational therapy background, and participants are all English speaking. These factors will have influenced the findings in such a way as to limit generalisation beyond New Zealand, and relevance to people who do not speak English. Additionally, beliefs and attitudes towards pain and coping are context-bound. In New Zealand there has been some attention in the media to remaining active despite pain, but surveys about pain beliefs in New Zealand suggest that many people believe that pain indicates tissue 
damage (Brown, Dean, Hay-Smith, Taylor, \& Baxter, 2010), complicated by misinterpreting what clinicians have said to them about their pain (Darlow et al., 2013). This has a direct impact on the importance participants placed on learning that their pain was unrelated to harm. Over time these beliefs may change and the relevance of this factor may reduce. A caveat with respect to how well findings apply in alternative contexts applies to all research whether quantitative or qualitative, and individuals reading this work will need to be aware of shifts in community attitudes and beliefs before applying the findings to other settings or populations.

\section{Recalling coping strategies}

I asked participants to recall the ways they coped with pain both during the interview and when completing standardised questionnaires, but there is no way to verify the answers. It is difficult to think of ways to mitigate this problem in my study. Participants found it hard to identify exactly how they coped with their pain, often saying things like "I just do it", "I just get on with it", "It just had to be done so I did it".

While a prospective study would counter this in some ways, it also requires a longitudinal cohort study and both the sample size and time needed would make this a complex undertaking. Only a small number of people who experience acute pain go on to develop chronic pain; even if a study is limited to individuals with a diagnosis of ankylosing spondylitis and rheumatoid arthritis, the total number of people with these diagnoses is relatively small compared to the number of people presenting to primary care with similar symptoms; additionally, a minority of those developing chronic pain demonstrate the resilience that is the characteristic under scrutiny in this study.

A second approach to mitigate the loss of recall accuracy particularly for coping strategies could be to ask individuals to participate in a daily process study (Schwarz, 2007; Sorbi et al., 2006a, 2006b). This approach involves using either pen and paper diary, or electronic 
prompts to record the coping strategy used at that time. There are advantages to this methodology, but it requires commitment by participants, and in the case of electronic prompts, equipment that is costly. There is also some evidence that coping strategies identified through daily process studies may provide different information about coping than that obtained via recall (Folkman \& Moskowitz, 2004).

Daily process methodology does not, however, deal with the problem of how to identify changes in perceptions or attitudes towards pain. An aim of cognitive behavioural approaches to chronic pain helping people reconceptualise their pain as less threatening. This approach also helps individuals develop a more flexible approach to managing their pain than avoiding it (Day et al., 2012). The participants in my study had not attended pain management; thus their interpretations and cognitive coping strategies have developed independently of healthcare providers, meaning the participants rarely had names for the ways they coped. As noted above, cognitive coping tends to be recalled less frequently than behavioural coping approaches (Stone et al., 1998).

\section{Recalling diagnosis}

There is also the possibility of recall bias relating to participant's recollections of their diagnosis. As mentioned above, a prospective study design is the only alternative to asking participants to remember what occurred. As a result, there is a risk of recall bias such as remembering only salient events rather than typical care, being influenced by current mood state or even responding to cues from contextual factors such as the questions asked just prior to a question. It is hypothesised that people make an estimate based on what they can remember and their overall beliefs or attitudes towards what they are being asked about (Smith, Brown, et al., 2008). This suggests some caution should be applied when generalising findings from this study and further research to corroborate 
these findings is warranted. Despite advising caution, there are features of this study that suggest I have identified a consistent finding:

- I asked participants similar questions about the onset of their symptoms and their diagnosis. This maintained consistency of contextual cues.

- The time since diagnosis and becoming a participant in this study varied, with participants having between two years and 26 years since diagnosis.

- There was considerable consistency between the participant's descriptions of events despite individuals being different in terms of occupation, age, socioeconomic background, diagnosis and ethnicity.

- The last point supports my contention that I have saturated the categories, obtaining multiple indicators of the underlying variable: this is a key tenet of grounded theory methodology (Glaser, 1998, p. 141).

\section{Interviewer bias}

Human cognitive biases are always present, but not always apparent, especially not to the individual involved (Ruscio, 2010; Schwab, 2008). Researchers analysing qualitative data must consider how much the researcher's own perspective might have biased the findings. I have discussed the way in which classical grounded theory views prior knowledge and integrates literature that already exists. The methodology relies on constant comparison and patterning out (saturation) to ensure categories are not based on a limited number of incidents. I believe the categories and relationships that have emerged from the data represent stable phenomena, and that the resultant model fits and works to explain the participant's main concern. 


\section{Opportunities for further research}

A man may fulfil the object of his existence by asking a question he cannot answer, and attempting a task he cannot achieve.

- Oliver Wendell Holmes b. 1809; d. 1894.

It may be a truism that Doctoral candidates hope to change the world, but instead change only the world of their family; nevertheless there are some questions posed by the findings of this thesis that merit further exploration and I will discuss them in this section of the thesis.

Grounded theory's main methodological contribution is that by obtaining grounded data from their participants and integrating this with existing theory new explanations for social phenomenon are generated. I have identified that people who identify as living well with chronic pain do so because they have successfully resolved their main concern. While this theoretical explanation fits, works and has relevance within the substantive area of living well with chronic pain, there remain unanswered questions, and the model poses new hypotheses for testing.

The first question is whether the model in toto applies to other groups of people who have successfully adapted to chronic pain. Substantive theory refers to a proscribed domain while formal theory applies across different contexts. A formal theory means looking for new conceptual comparisons (Andrews, 2012), and extending the core category beyond the original substantive area. In my model, it may mean looking at those who do not identify that they live well with chronic pain, to determine how well the model explains their situation. It may also mean exploring the ways people who live well with other painful conditions manage this transition, such as those with spinal cord injury, osteoarthritis, low back pain or peripheral neuropathy. The model could also be applied to 
groups of individuals living well with other chronic health conditions, particularly to establish the role of engaging in valued occupations, and would begin to extend the substantive theory towards formal theory.

The next area to study in more depth is the nature of interactions between clinicians and patients at the point where people need diagnostic clarity and symptom understanding. The critical point is how clinicians can convey that pain is likely to remain. The literature examining clinician attitudes, effective approaches for conveying "bad news" and ways to teach clinicians how to give this news effectively is slim and appears to be an area in which further research is essential. This is particularly noteworthy given the increasing number of people predicted to need to learn to live with chronic health conditions.

Clinicians do not appear to have been given explicit training on the best way to broach the subject of how to learn to live with a chronic condition, and as this thesis points out, patients complain it is not handled well.

I discussed the limitations of asking participants to recall their coping strategies in the section above, and mentioned the use of ecological momentary assessment as a method for obtaining data in real time. This method may offer some complementary information about the ways people who live well with pain use coping strategies in daily life. In particular, it could provide a window into the contexts in which strategies are employed, answering "how" questions about coping rather than "what" questions. For this reason, although I chose not to use this approach in this study, it would be useful in future studies exploring daily life coping in people with chronic pain.

Two final aspects of coping with chronic pain should be considered in detail. While mindfulness has received research attention recently, the focus has been on sedentary, and fairly cognitively sophisticated methods of helping people learn the skills. Participants in this study did not use these approaches and instead took a pragmatic approach. They 
did not rely on meditation or sedentary ways of dealing with their thoughts about pain. Much of the clinical application of mindfulness has been derived from Kabat-Zinn's methodology (Kabat-Zinn, 1982), and it would be helpful to develop active, movementoriented approaches that enabled individuals with different learning styles to become skilled in the nonjudgemental awareness that characterises people who cope well. This may involve understanding flow (Csikszentmihalyi \& LeFevre, 1989), or immersion in activities or occupation (Elliot, 2011; Larson \& von Eye, 2010; Rebeiro \& Polgar, 1999; Reid, 2011), and developing therapeutic opportunities for this experience within occupation.

Secondly, it would be useful to determine whether individuals who cope well have an innate skill to be mindful in the context of valued occupations. Do they differ from individuals who cope less well in their ability to be mindful when engaging in valued occupations? Is the process of engaging in valued occupations a mechanism by which individuals can decouple pain-related appraisal and affect?

Several additional points should be examined in more detail. Interpersonal relationships, both in terms of emotional support and instrumental support, were not a strong feature of this study, and this is at odds with many other studies of coping with chronic health conditions including chronic pain. This finding may be an idiosyncratic characteristic of participants in this study, their place in the trajectory of coping with chronic pain, my questions, or it may be unique and novel to individuals who cope well with chronic pain. It would be helpful to understand more clearly how relevant interpersonal relationships are for people coping with chronic pain, particularly in light of the finding that relationships with trustworthy health professionals were influential in deciding.

I chose classical grounded theory because it is not aligned to any particular philosophical school yet is broadly compatible with a scientific realist perspective. This means the relationships between variables identified in the theory can be tested without violating 
philosophical assumptions of the methodology (unlike a constructivist approach), yet the grounded theory as a whole can stand on its empirical base. There are several predictions that can be derived from the resultant theory, however, that could be studied in greater detail to enable the concepts to be refined, and thus enable better use of the findings in a clinical setting. These relationships are:

- The relationship between occupational drive, and an individual's ideographic representation of pain, mediated by self-efficacy for occupational engagement;

- The relationship between distress, occupational drive, and accepting that hurt does not equal harm. Some participants described beginning to participate in valued occupations, but doing so with high levels of distress because they were not convinced that their pain did not indicate ongoing damage. This may explain why some people use a "boom and bust" approach to daily life, yet continue to be distressed, while people who cope well with pain use the same activity pattern but remain undistressed.

- The relationship between remaining in the first phase of making sense and the offer of temporary pain reduction via interventional procedures such as medial branch blocks and radiofrequency neurotomy. While my study demonstrated that individuals needed to know that their pain was unlikely to resolve completely, it did not examine the effect of ongoing interventions requiring repeated attendance at a clinical setting. This is an especially important question given that these methods have become common (Loeser, 2005b; Manchikanti, Pampati, Singh, \& Falco, 2013; Perret \& Rosen, 2011; Roth et al., 2012).

\section{Concluding remarks}

Chronic pain self-management is likely to be needed for many years to come because progress towards finding an effective pharmacological or medical approach is extremely 
slow. Greater numbers of people will develop chronic health conditions with painful sequelae as baby boomers in New Zealand reach old age. Although perhaps $30 \%$ of those with chronic pain manage their pain without help, $60 \%$ of people look to professionals for guidance. Health professionals feel an ethical obligation to offer relief to distressed individuals, but their knowledge of what does and does not work in daily life may be limited by the prevailing biomedical treatment model (Crawford, Ryan, \& Shipton, 2007), patient defensiveness about the relevance of psychosocial factors and limited research on how individuals use coping strategies within their own life context.

By studying people who identify as living well despite their pain, and in particular, studying those who are naïve to cognitive behavioural approaches to self-management, clinicians and researchers can begin to understand existing strategies and factors they might be able to influence or support when people first attend for treatment.

This study has identified that people develop effective coping as a way to remain engaged in occupations they value. They engage in valued occupations because this enables them to redevelop their positive self-concept, integrating chronic pain as just one of many facets of their identity, rather than being the defining feature. Furthermore, this study shows that the specific coping strategies individuals use matters less than the purpose for which they are being used, the functional outcome of using them, and that individuals have a range of approaches that they can draw on in a flexible manner.

This finding has many implications both for clinicians and researchers, providing both groups with support for adopting a functional contextual view of coping. Clinically, this suggests the ACT approach offers a great deal as a therapeutic approach, particularly for improving long-term function. If individuals can use many coping strategies rather than being limited to those deemed active or adaptive, they may then be able to draw on welllearned and familiar approaches rather than having to learn new skills. Well-learned and 
rehearsed skills are more easily recalled in stressful situations, and thus may require less effort to use. For research, this finding leads to many new research questions about metacognition, values-aligned activity, and coping with identity change.

The study explains how individuals accept chronic pain as part of their life. The process of learning to accept is important given the increasing acknowledgement of acceptance as a critical factor influencing both distress and disability associated with pain. The factors identified in this study are modifiable rather than being trait characteristics, and so provide options for therapeutic intervention. Attention can be paid to not only individuals with chronic pain, but also to the contextual factors that influence the trajectory. Clinician's behaviours are shown to have a strong influence on how quickly an individual can progress through the phase of making sense of pain towards flexibly persisting. Taking the time to provide explanations of diagnoses, the meaning of symptoms, prognosis and giving a sense of collaboration may provide individuals with the information they need before deciding. Even more importantly, advising patients that their problem is manageable rather than curable, and that seeking complete pain relief is unlikely to be successful has emerged as a critical factor.

Finally, the positive motivation derived from the desire to participate in important occupations is a factor that all clinicians, but particularly occupational therapists, must recognise. Occupations allow individuals to enact the values that are important to them thus being able re-occupy self. 


\section{References}

Abrahams, T. (2008). Occupation, identity and choice: a dynamic interaction. Journal of Occupational Science, 15(3), 186-189.

Affleck, G., Tennen, H., Zautra, A., Urrows, S., Abeles, M., \& Karoly, P. (2001 ). Women's pursuit of personal goals in daily life with fibromyalgia: a value-expectancy analysis. Journal of Consulting and Clinical Psychology, 69(4), 587-596. doi: dx.doi.org/10.1037//0022-006X.69.4.587

Affleck, Glenn, Urrows, Susan, Tennen, Howard, \& Higgins, Pamela. (1992). Daily coping with pain from rheumatoid arthritis: patterns and correlates. Pain, 51(2), 221-229. doi: 10.1016/0304-3959(92)90263-b

Akitsuki, Y., \& Decety, J. (2009). Social context and perceived agency affects empathy for pain: an event-related fMRI investigation. Neuroimage, 47(2), 722-734. doi: dx.doi.org/10.1016/j.neuroimage.2009.04.091

Allegretti, Andrew, Borkan, Jeffrey, Reis, Shmuel, \& Griffiths, Frances. (2010). Paired interviews of shared experiences around chronic low back pain: Classic mismatch between patients and their doctors. Family Practice, 27(6), 676-683. doi: http://dx.doi.org/10.1093/fampra/cmq063

Alsaker, S, \& Josephsson, S. (2010). Occupation and meaning: Narrative in everyday activities of women with chronic rheumatic conditions. OTJR Occupation Participation and Health, 30(2), 58-67. doi: dx.doi.org/10.3928/15394492-2010031201

Altschuler, Eric Lewin. (2006). Using ice cream headache to help physicians experience the pain of and empathize with cluster headache patients. Medical Hypotheses, 66(3), 685. doi: 10.1016/j.mehy.2005.09.039

Alvesson, M., \& Karreman, D. (2007). Constructing Mystery: Empirical matters in theory development. Academy of Management Review, 32(4), 1265-1281. doi: 10.5465/amr.2007.26586822

Anand, K. J. S., Rovnaghi, Cynthia, Walden, Marlene, \& Churchill, John. (1999). Consciousness, behavior, and clinical impact of the definition of pain. Pain Forum, 8(2), 64-73. doi: http://dx.doi.org/10.1016/S1082-3174(99)70029-2

Anand, K.J.S., \& Craig, K. D. (1996). Editorial. Pain, 67(1), 3-6. doi: http://dx.doi.org/10.1016/0304-3959(96)03135-1

Anderson, R., \& Hanrahan, S. J. (2008). Dancing in pain: pain appraisal and coping in dancers. Journal of Dance Medicine \& Science, 12(1), 9-16.

Andersson, H., Ejlertsson, Goran, Leden, Ido, \& Schersten, Bengt. (1999). Impact of chronic pain on health care seeking, self care, and medication. Results from a populationbased Swedish study. Journal of Epidemiology and Community Health, 53(8), 503509. doi: http://dx.doi.org/10.1136/jech.53.8.503 
Andersson, H. I. (2009). Increased mortality among individuals with chronic widespread pain relates to lifestyle factors: a prospective population-based study. Disability \& Rehabilitation, 31(24), 1980-1987. doi: 10.3109/09638280902874154

Andrews, Nicole E., Strong, Jenny, \& Meredith, Pamela J. (2012). Activity Pacing, Avoidance, Endurance, and Associations With Patient Functioning in Chronic Pain: A Systematic Review and Meta-Analysis. Archives of Physical Medicine and Rehabilitation, 93(11), 2109-2121.e2107. doi: dx.doi.org/10.1016/j.apmr.2012.05.029

Andrews, T. (2012). Reflections on generating a formal grounded theory. In V. B. Martin \& A. Gynnild (Eds.), Grounded Theory: The philosophy, method, and work of Barney Glaser. Boca Raton, Florida, USA: BrownWalker.

Ansary, Maged El, Steigerwald, Ilona, \& Esser, Stefan. (2003). Egypt: Over 5000 Years of Pain Management - Cultural and Historic Aspects. Pain Practice, 3(1), 84-87. doi: 10.1046/j.1533-2500.2003.00010.x

Antonovsky, Aaron. (1996). The salutogenic model as a theory to guide health promotion. Health Promotion International, 11(1), 11-18. doi: 10.1093/heapro/11.1.11

Arnold, Lesley M., Crofford, Leslie J., Mease, Philip J., Burgess, Somali Misra, Palmer, Susan C., Abetz, Linda, \& Martin, Susan A. (2008). Patient perspectives on the impact of fibromyalgia. Patient Education \& Counseling, 73(1), 114-120. doi: dx.doi.org/10.1016/j.pec.2008.06.005

Asante, A. K., Brintnell, E. S., \& Gross, D. P. (2007). Functional self-efficacy beliefs influence functional capacity evaluation. Journal of Occupational Rehabilitation, 17(1), 73-82. doi: 10.1007/s10926-007-9068-1

Asbring, Pia. (2001). Chronic illness - a disruption in life: identity-transformation among women with chronic fatigue syndrome and fibromyalgia. Journal of Advanced Nursing, 34(3), 312-319. doi: 10.1046/j.1365-2648.2001.01767.x

Asghari, A., \& Nicholas, M. K. (2001). Pain self-efficacy beliefs and pain behaviour. A prospective study. Pain, 94(1), 85-100. doi: dx.doi.org/10.1016/S03043959(01)00344-X

Ashby, Samantha, Fitzgerald, Maureen, \& Raine, Simon. (2012). The impact of chronic low back pain on leisure participation: implications for occupational therapy. British Journal of Occupational Therapy, 75(11), 503-508. doi: 10.4276/030802212X13522194759897

Asmundson, Gordon J. G. (2012). Do attentional biases for pain depend on threat value of pain and competing motivation toward non-pain goals? Pain, 153(6), 1140-1141. doi: http://dx.doi.org/10.1016/j.pain.2012.03.002

Asmundson, Gordon J. G., \& Katz, Joel. (2009). Understanding the co-occurrence of anxiety disorders and chronic pain: state-of-the-art. Depression and Anxiety, 26(10), 888-901. doi: 10.1002/da.20600

Asmundson, Gordon J., \& Hadjistavropoulos, Heather D. (2007). Is High Fear of Pain Associated With Attentional Biases for Pain-Related or General Threat? A Categorical Reanalysis. The Journal of Pain, 8(1), 11-18. doi: dx.doi.org/10.1016/j.jpain.2006.05.008 
Atkinson, P., Martin, C., \& Rankin, J. (2009). Resilience revisited. Journal of Psychiatric and Mental Health Nursing, 16(2), 137-145. doi: dx.doi.org/10.1111/j.13652850.2008.01341.x

Austin, James T., \& Vancouver, Jeffrey B. (1996). Goal constructs in psychology: Structure, process, and content. Psychological Bulletin 120(3), 338-375. doi: dx.doi.org/10.1037//0033-2909.120.3.338

Backman, K., \& Kyngäs, H.A. (1999). Challenges of the grounded theory approach to a novice researcher. Nursing \& Health Sciences, 1(3), 147-153. doi: dx.doi.org/10.1046/j.1442-2018.1999.00019.x

Bagraith, Karl S., Strong, Jenny, \& Sussex, Roland. (2012). Disentangling disability in the fear avoidance model: More than pain interference alone. The Clinical Journal of Pain, 28(3), 273-274. doi: dx.doi.org/10.1097/AJP.0b013e3182273e47

Bailey, Kristen M., Carleton, R., Vlaeyen, Johan W., \& Asmundson, Gordon J. (2010). Treatments addressing pain-related fear and anxiety in patients with chronic musculoskeletal pain: A preliminary review. Cognitive Behaviour Therapy, 39(1), 4663. doi: http://dx.doi.org/10.1080/16506070902980711

Baker, Susan C., Gallois, Cindy, Driedger, S., \& Santesso, Nancy. (2011). Communication accommodation and managing musculoskeletal disorders: Doctors' and patients' perspectives. Health Communication, 26(4), 379-388. doi: http://dx.doi.org/10.1080/10410236.2010.551583

Baldwin, M. L., \& Butler, R. J. (2006). Upper extremity disorders in the workplace: costs and outcomes beyond the first return to work. Journal of Occupational Rehabilitation, 16(3), 303-323. doi: dx.doi.org/10.1007/s10926-006-9043-2

Baliki, Marwan N., Geha, Paul Y., Apkarian, A., \& Chialvo, Dante R. (2008). Beyond feeling: Chronic pain hurts the brain, disrupting the default-mode network dynamics. The Journal of Neuroscience, 28(6), 1398-1403. doi: http://dx.doi.org/10.1523/JNEUROSCI.4123-07.2008

Ballinger, C. (2004). Writing Up Rigour: Representing and Evaluating Good Scholarship in Qualitative Research. British Journal of Occupational Therapy, 67(12), 540-546.

Baranoff, J., Hanrahan, S., Kapur, D., \& Connor, J. (2013). Acceptance as a process variable in relation to catastrophizing in multidisciplinary pain treatment. European Journal of Pain, 17(1), 101-110. doi: dx.doi.org/10.1002/j.1532-2149.2012.00165.x

Bargiel-Matusiewicz, K., \& Krzyszkowska, A. (2009). Dispositional optimism and coping with pain. European Journal of Medical Research, 14 Supplement 4, 271-274. doi: dx.doi.org/10.1186/2047-783X-14-S4-271

Barker, Karen L., Reid, Margaret, \& Lowe, Catherine J. (2014). What does the language we use about arthritis mean to people who have osteoarthritis? A qualitative study. Disability and Rehabilitation: An International, Multidisciplinary Journal, 36(5), 367-372.

Batavia, A. I., DeJong, G., \& McKnew, L. B. (1991). Toward a national personal assistance program: the independent living model of long-term care for persons with disabilities. 
Journal of Health Politics, Policy \& Law, 16(3), 523-545. doi: dx.doi.org/10.1215/03616878-16-3-523

Baumeister, Roy F. (1997). Identity, self-concept, and self-esteem: The self lost and found. In R. Hogan, J. A. Johnson \& S. R. Briggs (Eds.), Handbook of personality psychology (pp. 681-710). San Diego, CA, US: Academic Press.

Beer, Nils, \& Moneta, Giovanni B. (2012). Coping and perceived stress as a function of positive metacognitions and positive meta-emotions. Individual Differences Research, $10(2), 105-116$.

Bensing, Jozien M., Deveugele, Myriam, Moretti, Francesca, Fletcher, Ian, van Vliet, Liesbeth, Van Bogaert, Marjolein, \& Rimondini, Michela. (2011). How to make the medical consultation more successful from a patient's perspective? Tips for doctors and patients from lay people in the United Kingdom, Italy, Belgium and the Netherlands. Patient Education and Counseling, 84(3), 287-293. doi: http://dx.doi.org/10.1016/j.pec.2011.06.008

Benuzzi, Francesca, Lui, Fausta, Duzzi, Davide, Nichelli, Paolo F., \& Porro, Carlo A. (2008). Does it look painful or disgusting? Ask your parietal and cingulate cortex. The Journal of Neuroscience, 28(4), 923-931. doi: http://dx.doi.org/10.1523/JNEUROSCI.4012-07.2008

Berry, Donna L., Wilkie, Diana J., Thomas, Charles R., Jr., \& Fortner, Paige. (2003). Clinicians communicating with patients experiencing cancer pain. Cancer Investigation, 21(3), 374-381. doi: dx.doi.org/10.1081/CNV-120018228

Bertakis, K, Azari, R, \& Callahan, E. (2003). Patient Pain: Its Influence on Primary Care Physician-Patient Interaction. Family Medicine Journal, 35(2), 119-123.

Betti, V., Zappasodi, F., Rossini, P. M., Aglioti, S. M., Tecchio, F., Betti, Viviana, . . . Tecchio, Franca. (2009). Synchronous with your feelings: sensorimotor \{gamma\} band and empathy for pain. Journal of Neuroscience, 29(40), 12384-12392.

Bickerton, D. (2003). Symbol and structure: a comprehensive framework for language evolution. Studies in the evolution of language, 3, 77-93. doi: dx.doi.org/10.1093/acprof:oso/9780199244843.003.0005

Birkholtz, M., Aylwin, L., \& Harman, R. M. (2004 ). Activity Pacing in Chronic Pain Management: One Aim, but Which Method? Part Two: National Activity Pacing Survey. British Journal of Occupational Therapy, 67(11), 481-487.

Bishop, Scott R., Lau, Mark, Shapiro, Shauna, Carlson, Linda, Anderson, Nicole D., Carmody, James, . . . Devins, Gerald. (2004). Mindfulness: A proposed operational definition. Clinical Psychology: Science and Practice, 11(3), 230-241. doi: dx.doi.org/10.1093/clipsy.bph077

Block, C. K., \& Brock, J. (2008). The relationship of pain catastrophizing to heightened feelings of distress. Pain Management Nursing, 9(2), 73-80. doi: dx.doi.org/10.1016/j.pmn.2008.01.004

Blumer, H. (1980). Mead and Blumer: The convergent methodological perspectives of social behaviorism and symbolic interactionism. American Sociological Review, 409-419. doi: dx.doi.org/10.2307/2095174 
Blyth, Fiona M., Macfarlane, Gary J., \& Nicholas, Michael K. (2007). The contribution of psychosocial factors to the development of chronic pain: The key to better outcomes for patients? Pain, 129(1-2), 8-11. doi: http://dx.doi.org/10.1016/j.pain.2007.03.009

Bonica, John Joseph, \& Loeser, John D. (2001). History of Pain Concepts and Therapies. In J. D. Loeser (Ed.), Bonica's Management of Pain (Third ed., Vol. 1, pp. 3-16). Philadelphia: Lippincott, Williams and Wilkins.

Bonvicini, K.A., Perlin, M.J., Bylund, C.L., Carroll, G., Rouse, R.A., \& Goldstein, M.G. (2009). Impact of communication training on physician expression of empathy in patient encounters. Patient Education and Counseling, 75(1), 3-10. doi: dx.doi.org/10.1016/j.pec.2008.09.007

Borell, L., Asaba, E., Rosenberg, L., Schult, M. L., \& Townsend, E. (2006). Exploring experiences of "participation" among individuals living with chronic pain. Scandinavian Journal of Occupational Therapy, 13(2), 76-85. doi: 10.1080/11038120600673023

Borkan, Jeffrey , Van Tulder, Maurits , Reis, Shmuel, Schoene, Mark L. , Croft, Peter , \& Hermoni, Doron (2002). Advances in the Field of Low Back Pain in Primary Care: A Report from the Fourth International Forum. Spine, 27(5), E128-E132. doi: dx.doi.org/10.1097/00007632-200203010-00019

Borkum, Jonathan M. (2010). Maladaptive cognitions and chronic pain: Epidemiology, neurobiology, and treatment. Journal of Rational-Emotive \& Cognitive Behavior Therapy, 28(1), 4-24. doi: http://dx.doi.org/10.1007/s10942-010-0109-x

Borsbo, B., Peolsson, M., \& Gerdle, B. (2009). The complex interplay between pain intensity, depression, anxiety and catastrophising with respect to quality of life and disability. Disability \& Rehabilitation, 31(19), 1605-1613. doi: dx.doi.org/10.1080/09638280903110079

Bourne, R. B., Chesworth, B. M., Davis, A. M., Mahomed, N. N., \& Charron, K. D. (2010). Patient satisfaction after total knee arthroplasty: who is satisfied and who is not? Clinical Orthopaedics \& Related Research, 468(1), 57-63. doi: dx.doi.org/10.1007/s11999-009-1119-9

Bowey-Morris, Julia, Davis, Sarah, Purcell-Jones, Gari, \& Watson, Paul J. (2011). Beliefs about back pain: Results of a population survey of working age adults. The Clinical Journal of Pain, 27(3), 214-224.

Bozic, Kevin J, Kurtz, Steven M, Lau, Edmund, Ong, Kevin, Chiu, Vanessa, Vail, Thomas P, ... Berry, Daniel J. (2010). The Epidemiology of Revision Total Knee Arthroplasty in the United States. Clinical Orthopaedics and Related Research, 468(1), 45-51. doi: 10.1007/s11999-009-0945-0

Branch, William T., Jr., Davis, Kelli, \& Weng, Mark. (2012). Teaching compassion. Patient Education \& Counseling, 89(1), 3-4. doi: dx.doi.org/10.1016/j.pec.2012.03.013

Brandtstädter, Jochen. (2009). Goal pursuit and goal adjustment: Self-regulation and intentional self-development in changing developmental contexts. Advances in Life Course Research, 14(1-2), 52-62. doi: http://dx.doi.org/10.1016/j.alcr.2009.03.002 
Branstetter-Rost, Ann, Cushing, Christopher, \& Douleh, Tanya. (2009). Personal values and pain tolerance: Does a values intervention add to acceptance? The Journal of Pain, 10(8), 887-892. doi: 10.1016/j.jpain.2009.01.001

Breen, A., Austin, H., Campion-Smith, C., Carr, E., \& Mann, E. (2007). "You feel so hopeless": a qualitative study of GP management of acute back pain. European Journal of Pain: Ejp, 11(1), 21-29. doi: dx.doi.org/10.1016/j.acpain.2007.02.021

Breivik, Harald, Collett, Beverly, Ventafridda, Vittorio, Cohen, Rob, \& Gallacher, Derek. (2006). Survey of chronic pain in Europe: prevalence, impact on daily life, and treatment. European Journal of Pain, 10(4), 287-287. doi: dx.doi.org/10.1016/j.ejpain.2005.06.009

Brekke, Mette, Hjortdahl, Per, \& Kvien, Tore K. (2002). Severity of musculoskeletal pain: Relations to socioeconomic inequality. Social Science \& Medicine, 54(2), 221-228. doi: http://dx.doi.org/10.1016/S0277-9536\%2801\%2900018-1

Brennan, Frank, Carr, Daniel B, \& Cousins, Michael. (2007). Pain management: a fundamental human right. Anesthesia \& Analgesia, 105(1), 205-221. doi: dx.doi.org/10.1213/01.ane.0000268145.52345.55

Bringer, Joy D., Johnston, Lynne Halley, \& Brackenridge, Celia H. (2006). Using ComputerAssisted Qualitative Data Analysis Software to Develop a Grounded Theory Project. Field Methods, 18(3), 245-266. doi: http://dx.doi.org/10.1177/1525822X06287602

Bringer, Joy D., Johnstone, Lynne, \& Brackenridge, C.H. (2004). Maximizing transparency in a doctoral thesis: The complexities of writing about the use of QSR* NVIVO within a grounded theory study. Qualitative Research, 4(2), 247-265.

Britt, Thomas W., Dickinson, James M., Moore, DeWayne, Castro, Carl A., \& Adler, Amy B. (2007). Correlates and Consequences of Morale Versus Depression Under Stressful Conditions. Journal of Occupational Health Psychology, 12(1), 34-47. doi: dx.doi.org/10.1037/1076-8998.12.1.34

Broadbent, Elizabeth. (2010). Illness perceptions and health: Innovations and clinical applications. Social and Personality Psychology Compass, 4(4), 256-266. doi: dx.doi.org/10.1111/j.1751-9004.2010.00260.x

Brown, Gregory K., \& Nicassio, Perry M. (1987). Development of a questionnaire for the assessment of active and passive coping strategies in chronic pain patients. Pain, 31(1), 53-64. doi: http://dx.doi.org/10.1016/0304-3959\%2887\%2990006-6

Brown, M., Dean, S., Hay-Smith, E. J. C., Taylor, W., \& Baxter, G. D. (2010). Musculoskeletal pain and treatment choice: an exploration of illness perceptions and choices of conventional or complementary therapies. Disability \& Rehabilitation, 32(20), 1645-1657. doi: 10.3109/09638281003649896

Bryman, A., \& Bell, E. (2011). Business research methods (3rd ed.): Oxford University Press, USA.

Buchbinder, R., \& Jolley, D. (2005). Effects of a media campaign on back beliefs is sustained 3 years after its cessation. Spine, 30(11), 1323-1330. doi: dx.doi.org/10.1097/01.brs.0000164121.77862.4b 
Buchbinder, R., Jolley, D., \& Wyatt, M. (2001). 2001 Volvo Award Winner in Clinical Studies: Effects of a media campaign on back pain beliefs and its potential influence on management of low back pain in general practice. Spine, 26(23), 2535-2542. doi: dx.doi.org/10.1097/00007632-200112010-00005

Buenaver, L.F., McGuire, L., \& Haythornthwaite, J.A. (2006). Cognitive-Behavioral selfhelp for chronic pain. Journal of Clinical Psychology, 62(11), 1389-1396. doi: dx.doi.org/10.1002/jclp.20318

Buhrman, Monica, Skoglund, Astrid, Husell, Josefin, Bergström, Kristina, Gordh, Torsten, Hursti, Timo, ... Andersson, Gerhard. (2013). Guided internet-delivered acceptance and commitment therapy for chronic pain patients: A randomized controlled trial. Behaviour Research and Therapy, 51(6), 307-315. doi: http://dx.doi.org/10.1016/j.brat.2013.02.010

Burnett, Angus, Sze, Ching Ching, Tam, Suet May, Yeung, Ka Man, Leong, Michelle, Wang, Wendy T., ... O'Sullivan, Peter. (2009). A cross-cultural study of the back pain beliefs of female undergraduate healthcare students. The Clinical Journal of Pain, 25(1), 20-28. doi: http://dx.doi.org/10.1097/AJP.0b013e3181805a1e

Burns, John W. (2006). Arousal of negative emotions and symptom-specific reactivity in chronic low back pain patients. Emotion, 6(2), 309-319. doi: http://dx.doi.org/10.1037/1528-3542.6.2.309

Cai, C., Pua, Y., \& Kian, C. (2008). Psychosocial differences between high-risk acute vs. chronic low back pain patients. Pain Practice, 8(2), 91-97.

Callister, Lynn Clark, Khalaf, Inaam, Semenic, Sonia, Kartchner, Robin, \& VehvilainenJulkunen, Katri. (2003). The pain of childbirth: perceptions of culturally diverse women. Pain Management Nursing, 4(4), 145-154. doi: 10.1016/s15249042(03)00028-6

Campbell, Carol, \& Cramb, Georgia. (2008). 'Nobody likes a back bore'--Exploring lay perspectives of chronic pain: Revealing the hidden voices of nonservice users. Scandinavian Journal of Caring Sciences, 22(3), 383-390. doi: dx.doi.org/10.1111/j.1471-6712.2007.00541.x

Cano, Annmarie, Miller, Lisa Renee, \& Loree, Amy. (2009). Spouse beliefs about partner chronic pain. The Journal of Pain, 10(5), 486-492. doi: http://dx.doi.org/10.1016/j.jpain.2008.11.005

Car, J., \& Sheikh, A. (2004). An Evidence-Based Approach to Low Back Pain in Primary Care. Advanced Studies in Medicine 4(3), 135-148 + 160.

Cardol, Mieke, van den Bosch, Wil J., Spreeuwenberg, Peter, Groenewegen, Peter P., van Dijk, Liset, \& de Bakker, Dinny H. (2006). All in the Family: Headaches and Abdominal Pain as Indicators for Consultation Patterns in Families. Annals of Family Medicine, 4(6), 506-511. doi: http://dx.doi.org/10.1370/afm.584

Carr, Daniel B., \& Goudas, Leonidas C. (1999). Acute pain. The Lancet, 353(9169), 20512058. doi: http://dx.doi.org/10.1016/S0140-6736(99)03313-9 
Carroll, Linda J., Rothe, J. Peter, \& Ozegovic, Dejan. (2013). What does coping mean to the worker with pain-related disability? A qualitative study. Disability \& Rehabilitation, 35(14), 1182-1190. doi: 10.3109/09638288.2012.723791

Cassell, Eric J. (1999). Diagnosing Suffering: A Perspective. Annals of Internal Medicine, 131(7), 531-534. doi: 10.7326/0003-4819-131-7-199910050-00009

Cassell, Eric J. (2011). Suffering, whole person care, and the goals of medicine. In T. A. E. Hutchinson (Ed.), Whole person care: A new paradigm for the 21st century (pp. 922). New York, NY: Springer. Retrieved from http://ovidsp.ovid.com/ovidweb.cgi? T=JS\&CSC=Y\&NEWS=N\&PAGE=fulltext\&D= psyc7\&AN=2011-24010-002. doi: 10.1007/978-1-4419-9440-0

Cedraschi, C., \& Allaz, A. F. (2005). How to identify patients with a poor prognosis in daily clinical practice. Best Practice \& Research in Clinical Rheumatology, 19(4), 577-591. doi: dx.doi.org/10.1016/j.berh.2005.03.002

Chalk, H. M. (2007). Mind over matter: cognitive-behavioral determinants of emotional distress in multiple sclerosis patients. Psychology, Health \& Medicine, 12(5), 556566. doi: dx.doi.org/10.1080/13548500701244965

Chapman, C. Richard, \& Gavrin, Jonathan. (1999). Suffering: the contributions of persistent pain. The Lancet, 353(9171), 2233-2237. doi: http://dx.doi.org/10.1016/S01406736(99)01308-2

Charmaz, K. (2006a). Constructing Grounded Theory: A practical guide through qualitative analysis. London: Sage Publications.

Charmaz, K. (2002). The self as habit: The reconstruction of self in chronic illness. Occupational Therapy Journal of Research, 22(Suppl1), 31S-41S. doi: dx.doi.org/10.1177/15394492020220S105

Charmaz, K. (2006b). Constructing grounded theory: A practical guide through qualitative analysis: Sage Publications Limited.

Charmaz, Kathy. (1995). The Body, Identity, and Self: Adapting to Impairment. The Sociological Quarterly, 36(4), 657-680. doi: 10.2307/4121346

Charmaz, Kathy. (2006c). Measuring pursuits, marking self: Meaning construction in chronic illness. International Journal of Qualitative Studies on Health and Well-being, 1(1), 27-37. doi: dx.doi.org/10.1080/17482620500534488

Chibnall, J. T., \& Tait, R. C. (2009). Long-term adjustment to work-related low back pain: associations with socio-demographics, claim processes, and post-settlement adjustment. Pain Medicine, 10(8), 1378-1388. doi: 10.1111/j.15264637.2009.00738.x

Chiesa, Alberto, \& Serretti, Alessandro. (2011). Mindfulness-based interventions for chronic pain: A systematic review of the evidence. The Journal of Alternative and Complementary Medicine, 17(1), 83-93. doi: dx.doi.org/10.1089/acm.2009.0546

Chiros, Christine, \& O'Brien, William H. (2011). Acceptance, appraisals, and coping in relation to migraine headache: An evaluation of interrelationships using daily diary 
methods. Journal of Behavioral Medicine, 34(4), 307-320. doi: dx.doi.org/10.1007/s10865-011-9313-0

Christiansen, C. (2000). Identity, personal projects and happiness: self construction in everyday action. Journal of Occupational Science, 7(3), 98-107. doi: dx.doi.org/10.1080/14427591.2000.9686472

Christiansen, C. H. (1999). Defining lives: occupation as identity: an essay on competence, coherence, and the creation of meaning. British Journal of Occupational Therapy, 53(6), 547-558.

Chronister, J., \& Chan, F. (2007). Hierarchical Coping: A Conceptual Framework for Understanding Coping Within the Context of Chronic Illness and Disability. In E. Martz, H. Livneh \& B. Wright (Eds.), Coping with Chronic Illness and Disability: Theoretical, Empirical, and Clinical Aspects (pp. 49-71). New York: Springer Science + Business Media.

Ciechanowski, P., Sullivan, M., Jensen, M., Romano, J., \& Summers, H. (2003). The relationship of attachment style to depression, catastrophizing and health care utilization in patients with chronic pain. Pain, 104(3), 627-637. doi: dx.doi.org/10.1016/S0304-3959(03)00120-9

Clarke, Kathryn A, \& Iphofen, Ron. (2007). Accepting pain management or seeking pain cure: An exploration of patients' attitudes to chronic pain. Pain Management Nursing, 8(2), 102-110. doi: dx.doi.org/10.1016/j.pmn.2007.03.006

Cohen, L., Manion, L., \& Morrison, K. (2011). Research Methods in Education L. Cohen, L. Manion \& K. Morrison (Eds.),

Committee, National Ethics Advisory. (2012). Ethical Guidelines for Observational Studies: Observational research, audits and related activities. Revised edition. Wellington: Ministry of Health Retrieved from http://neac.health.govt.nz/sites/neac.health.govt.nz/files/documents/publications/ethic al-guidelines-for-intervention-studies-2012v2_0.doc.

Corbiere, Marc, Sullivan, Michael J., Stanish, William D., \& Adams, Heather. (2007). Pain and depression in injured workers and their return to work: A longitudinal study. Canadian Journal of Behavioural Science/Revue canadienne des Sciences du comportement 39(1), 23-31. doi: dx.doi.org/10.1037/cjbs2007002

Corbin, J.M., \& Strauss, A. (1990a). Grounded theory research: Procedures, canons, and evaluative criteria. Qualitative sociology, 13(1), 3-21.

Corbin, J.M., \& Strauss, A. (1991). A nursing model for chronic illness management based upon the trajectory framework. Research and Theory for Nursing Practice, 5(3), 155174.

Corbin, Juliet , \& Strauss, Anselm (2008a). Introduction Basics of Qualitative Research (3rd ed.): Techniques and Procedures for Developing Grounded Theory. (pp. 1-19). Thousand Oaks, CA: SAGE Publications, Inc.

Corbin, Juliet, \& Strauss, Anselm. (1990b). Grounded Theory Research: Procedures, Canons, and Evaluative Criteria. Qualitative Sociology, 13(1), 3. 
Corbin, Juliet, \& Strauss, Anselm. (2008b). Basics of qualitative research: Techniques and procedures for developing grounded theory (3 ed.). Thousand Oaks: Sage.

Costa, Joana, \& Pinto-Gouveia, Jose. (2013). Experiential avoidance and self-compassion in chronic pain. Journal of Applied Social Psychology, 43(8), 1578-1591. doi: dx.doi.org/10.1111/jasp.12107

Cote, James E. (1997). A Social History of Youth in Samoa. International Journal of Comparative Sociology, 38(3-4), 3-4.

Cote, Pierre, Cassidy, J., \& Carroll, Linda. (2001). The treatment of neck and low back pain: Who seeks care? Who goes where? Medical Care, 39(9), 956-967. doi: http://dx.doi.org/10.1097/00005650-200109000-00006

Covington, Edward C. (2000). Psychogenic Pain-What It Means, Why It Does Not Exist, and How to Diagnose It. Pain Medicine, 1(4), 287-294. doi: 10.1046/j.15264637.2000.00049.x

Crawford, C., Ryan, K., \& Shipton, E. (2007). Exploring general practitioner identification and management of psychosocial Yellow Flags in acute low back pain. New Zealand Medical Journal, 120(1254), U2536.

Creswell, J.W., Hanson, W.E., Plano, V.L.C., \& Morales, A. (2007). Qualitative Research Designs Selection and Implementation. The Counseling Psychologist, 35(2), 236-264. doi: dx.doi.org/10.1177/0011000006287390

Crombez, G., Vlaeyen, J. W., Heuts, P. H., \& Lysens, R. (1999). Pain-related fear is more disabling than pain itself: evidence on the role of pain-related fear in chronic back pain disability. Pain., 80(1-2), 329-339. doi: dx.doi.org/10.1016/S03043959(98)00229-2

Crombez, Geert, Eccleston, Christopher, Van Damme, Stefaan, Vlaeyen, Johan W., \& Karoly, Paul. (2012). Fear-avoidance model of chronic pain: The next generation. The Clinical Journal of Pain, 28(6), 475-483.

Crombez, Geert, Viane, Ilse, Eccleston, Christopher, Devulder, Jacques, \& Goubert, Liesbet. (2013). Attention to pain and fear of pain in patients with chronic pain. Journal of Behavioral Medicine, 36(4), 371-378. doi: dx.doi.org/10.1007/s10865-012-9433-1

Cronje, Ruth J., \& Williamson, Owen D. (2006). Is Pain Ever "Normal?". The Clinical Journal of Pain, 22(8), 692-699.

Crowley-Matoka, M., Saha, S., Dobscha, S. K., \& Burgess, D. J. (2009). Problems of quality and equity in pain management: exploring the role of biomedical culture. Pain Medicine, 10(7), 1312-1324. doi: 10.1111/j.1526-4637.2009.00716.x

Csikszentmihalyi, Mihaly, \& LeFevre, Judith. (1989). Optimal experience in work and leisure. Journal of personality and social psychology, 56(5), 815. doi: dx.doi.org/10.1037//0022-3514.56.5.815

Curran, Charlotte, Williams, Amanda C., \& Potts, Henry W. (2009). Cognitive-behavioral therapy for persistent pain: Does adherence after treatment affect outcome? European Journal of Pain, 13(2), 178-188. doi: http://dx.doi.org/10.1016/j.ejpain.2008.06.009 
Curry, T. J., \& Strauss, R. H. (1994). A little pain never hurt anybody; a photo-essay on the normalization of sport injuries. / Une petite douleur ne fait de mal a personne: etude visuelle sur la normalisation des blessures en sport. Sociology of Sport Journal, 11(2), 195-208.

Dahl, JoAnne, \& Lundgren, Tobias. (2006). Acceptance and Commitment Therapy (ACT) in the Treatment of Chronic Pain. . In R. A. Baer (Ed.), Mindfulness-based treatment approaches: Clinician's guide to evidence base and applications (pp. 285-306). San Diego, CA: Elsevier Academic Press.

Damasio, Antonio R., Everitt, B. J., \& Bishop, D. (1996). The Somatic Marker Hypothesis and the Possible Functions of the Prefrontal Cortex [and Discussion]. Philosophical Transactions of the Royal Society of London. Series B: Biological Sciences, 351(1346), 1413-1420. doi: 10.1098/rstb.1996.0125

Damsgard, E., Dewar, A., Roe, C., \& Hamran, T. (2011). Staying active despite pain: Pain beliefs and experiences with activity-related pain in patients with chronic musculoskeletal pain. Scandinavian Journal of Caring Sciences, 25(1), 108-116. doi: 10.1111/j.1471-6712.2010.00798.x

Darlow, Ben, Dowell, Anthony, Baxter, G. David, Mathieson, Fiona, Perry, Meredith, \& Dean, Sarah. (2013). The Enduring Impact of What Clinicians Say to People With Low Back Pain. Annals of Family Medicine, 11(6), 527-534. doi: 10.1370/afm.1518

Dasch, Burkhard, Endres, Heinz G., Maier, Christoph, Lungenhausen, Margitta, Smektala, Rudiger, Trampisch, Hans J., \& Pientka, Ludger. (2008). Fracture-related hip pain in elderly patients with proximal femoral fracture after discharge from stationary treatment. European Journal of Pain, 12(2), 149-156. doi: dx.doi.org/10.1016/j.ejpain.2007.03.012

Davis, D., Baddock, S., Pairman, S., Hunter, M., Benn, C., Wilson, D., . . Herbison, P. (2011). Planned place of birth in New Zealand: does it affect mode of birth and intervention rates among low-risk women? Birth, 38(2), 111-119. doi: http://dx.doi.org/10.1111/j.1523-536X.2010.00458.x

Day, Melissa A. , Thorn, Beverly E. , \& Burns, John W. . (2012). The Continuing Evolution of Biopsychosocial Interventions for Chronic Pain. Journal of Cognitive Psychotherapy, 26(2), 114-129. doi: 10.1126/science.847460.1978-0142300110.1126/

de Jong, J. R., Vlaeyen, J. W., Onghena, P., Goossens, M. E., Geilen, M., \& Mulder, H. (2005). Fear of movement/(re)injury in chronic low back pain: education or exposure in vivo as mediator to fear reduction? Clinical Journal of Pain, 21(1), 9-17. doi: dx.doi.org/10.1097/00002508-200501000-00002

de Ridder, D., \& Schreurs, K. (2001). Developing interventions for chronically ill patients: is coping a helpful concept? Clinical Psychology Review, 21(2), 205-240.

DeJong, G. (1979). Independent living: from social movement to analytic paradigm. Archives of Physical Medicine \& Rehabilitation, 60(10), 435-446.

Delahaij, Roos, van Dam, Karen, Gaillard, Anthony W., \& Soeters, Joseph. (2011). Predicting performance under acute stress: The role of individual characteristics. 
International Journal of Stress Management, 18(1), 49-66. doi: dx.doi.org/10.1037/a0020891

Denscombe, M. (2007). The good research guide for small-scale social research projects (4 ed.). Berkshire, England: Open University Press.

Diefenbach, Michael A, \& Leventhal, Howard. (1996). The common-sense model of illness representation: Theoretical and practical considerations. Journal of Social Distress and the Homeless, 5(1), 11-38.

Dixon, Anna, Hibbard, Judith, \& Tusler, Martin. (2009). How do people with different levels of activation self-manage their chronic conditions? The Patient: Patient-Centered Outcomes Research, 2(4), 257-268.

Dolin, Simon J., de, C. Williams, A, Ashford, Nicholas, George, Julie, Pereira, Liz, \& Perello, Antonio. (2003). Factors affecting medical decision-making in patients with osteoarthritis of the hip: Allocation of surgical priority. Disability and Rehabilitation: An International, Multidisciplinary Journal, 25(14), 771-777. doi: dx.doi.org/10.1080/0963828031000090551

Dominick, C., Blyth, F., \& Nicholas, M. (2011). Patterns of chronic pain in the New Zealand population. New Zealand Medical Journal, 124(1337), 63-76.

Douglas, Heather, \& Magnus, PD. (2013). State of the Field: Why novel prediction matters. Studies in History and Philosophy of Science Part A, 44(4), 580-589. doi: dx.doi.org/10.1016/j.shpsa.2013.04.001

Downey, G. (2007). Producing Pain Techniques and Technologies in No-Holds-Barred Fighting. Social Studies of Science, 37(2), 201-226. doi: 10.1177/0306312706072174

Drapeau, Aline, Marchand, Alain, \& Beaulieu-Prévost, Dominic. (2011). Epidemiology of Psychological Distress. In P. L. LAbate (Ed.), Mental Illnesses - Understanding, prediction and control (pp. 105-134): InTech. Retrieved from http://www.intechopen.com/books/mental-illnesses-understanding-predictionandcontrol/epidemiology-of-psychological-distress.

Dunkel Schetter, Christine, \& Dolbier, Christyn. (2011). Resilience in the context of chronic stress and health in adults. Social and Personality Psychology Compass, 5(9), 634652. doi: http://dx.doi.org/10.1111/j.1751-9004.2011.00379.x

Dunn, Dana S., \& Dougherty, Sarah B. (2005). Prospects for a Positive Psychology of Rehabilitation. Rehabilitation Psychology, 50(3), 305-311. doi: dx.doi.org/10.1037/0090-5550.50.3.305

Dysvik, Elin, Natvig, Gerd Karin, Eikeland, Ole-Johan, \& Lindstrom, Torill Christine. (2005). Coping with chronic pain. International Journal of Nursing Studies, 42(3), 297-305. doi: dx.doi.org/10.1016/j.ijnurstu.2004.06.009

Eakin, E., Reeves, M., Winkler, E., Healy, G., Dunstan, D., Owen, N., . . Wilkie, K. (2013). Six-month outcomes from living well with diabetes: A randomized trial of a telephone-delivered weight loss and physical activity intervention to improve glycemic control. Annals of Behavioral Medicine, 46(2), 193-203. doi: dx.doi.org/10.1007/s12160-013-9498-2 
Eccleston, Christopher, \& Crombez, Geert. (2007). Worry and chronic pain: A misdirected problem solving model. Pain, 132(3), 233-236. doi:

http://dx.doi.org/10.1016/j.pain.2007.09.014

. The economic cost of arthritis in New Zealand in 2010. (2010) (pp. 79). Canberra: Report for Arthritis New Zealand.

Edwards, R. R., Kronfli, T., Haythornthwaite, J. A., Smith, M. T., McGuire, L., \& Page, G. G. (2008). Association of catastrophizing with interleukin-6 responses to acute pain. Pain, 140(1), 135-144. doi: dx.doi.org/10.1016/j.pain.2008.07.024

Edwards, Robert R., Campbell, Claudia, Jamison, Robert N., \& Wiech, Katja. (2009). The neurobiological underpinnings of coping with pain. Current Directions in Psychological Science, 18(4), 237-241. doi: http://dx.doi.org/10.1111/j.1467$\underline{8721.2009 .01643 . \mathrm{x}}$

Edwards, Robert R., Smith, Michael T., Kudel, Ian, \& Haythornthwaite, Jennifer. (2006). Pain-related catastrophizing as a risk factor for suicidal ideation in chronic pain. Pain, 126(1-3), 272-279. doi: dx.doi.org/10.1016/j.pain.2006.07.004

Egan, T. Marshall. (2002). Grounded Theory Research and Theory Building. Advances in Developing Human Resources, 4(3), 277-295. doi: 10.1177/1523422302043004

Eifert, Georg H., Thompson, Risa N., Zvolensky, Michael J., Edwards, Kimberly, Frazer, Nicole L., Haddad, John W., \& Davig, James. (2000). The Cardiac Anxiety Questionnaire: development and preliminary validity. Behaviour Research and Therapy, 38(10), 1039-1053. doi: dx.doi.org/10.1016/S0005-7967(99)00132-1

Elliot, Michelle L. (2011). Being Mindful about Mindfulness: An Invitation to Extend Occupational Engagement into the Growing Mindfulness Discourse. Journal of Occupational Science, 18(4), 366-376. doi: 10.1080/14427591.2011.610777

Elliott, N, \& Lazenbatt, A. (2005). How to recognise a 'quality' grounded theory research study. Australian Journal of Advanced Nursing, 22(3), 48-52.

Engel, George L. (1977). The need for a new medical model: A challenge for biomedicine. Science, 196(4286), 129-136. doi: http://dx.doi.org/10.1126/science.847460

Engel, George L. (1994). Psychogenic Pain and the Pain-Prone Patient. Psychological vulnerability to chronic pain (pp. 179-221). New York, NY: Springer Publishing Co; US.

Epker, J., \& Gatchel, R. J. (2000). Prediction of treatment-seeking behavior in acute TMD patients: practical application in clinical settings. Journal of Orofacial Pain, 14(4), 303-309.

Eriksen, Jorgen, Ekholm, Ola, Sjogren, Per, \& Rasmussen, Niels K. (2004). Development of and recovery from long-term pain. A 6-year follow-up study of a cross-section of the adult Danish population. Pain, 108(1-2), 154-162. doi:

dx.doi.org/10.1016/j.pain.2003.12.018

Eriksson, Monica, \& Lindström, Bengt. (2005). Validity of Antonovsky's sense of coherence scale: a systematic review. Journal of Epidemiology and Community Health, 59(6), 460-466. doi: 10.1136/jech.2003.018085 
Esteve, R., Ramirez-Maestre, C., \& Lopez-Martinez, A. (2012). Experiential avoidance and anxiety sensitivity as dispositional variables and their relationship to the adjustment to chronic pain. European Journal of Pain, 16(5), 718-726. doi:

dx.doi.org/10.1002/j.1532-2149.2011.00035.x

Esteve, Rosa, Ramirez-Maestre, Carmen, \& Lopez-Martinez, Alicia E. (2007). Adjustment to chronic pain: The role of pain acceptance, coping strategies, and pain-related cognitions. Annals of Behavioral Medicine, 33(2), 179-188. doi: http://dx.doi.org/10.1007/BF02879899

Evers, Andrea W., Kraaimaat, Floris W., Geene, Rinie, Jacobs, Johannes W., \& Bijlsma, Johannes W. (2003). Pain coping and social support as predictors of long-term functional disability and pain in early rheumatoid arthritis. Behaviour Research and Therapy, 41(11), 1295-1310. doi: dx.doi.org/10.1016/S0005-7967(03)00036-6

Evers, Andrea WM, Kraaimaat, Floris W, van Lankveld, Wim, Jongen, Peter JH, Jacobs, Johannes WG, \& Bijlsma, Johannes WJ. (2001). Beyond unfavorable thinking: the illness cognition questionnaire for chronic diseases. Journal of Consulting and Clinical Psychology, 69(6), 1026. doi: 10.1037//0022-106X.69.6.1026

Evers, Jeanine C. (2010). From the Past into the Future. How Technological Developments Change Our Ways of Data Collection, Transcription and Analysis. Forum: Qualitative Social Research Sozialforschung, 12(1), 1-31.

Farmer, Steven A., Roter, Debra L., \& Higginson, Irene J. (2006). Chest pain: Communication of symptoms and history in a London emergency department. Patient Education and Counseling, 63(1-2), 138-144. doi: http://dx.doi.org/10.1016/j.pec.2005.09.009

Faull, K., \& Hills, M. (2006). The role of the spiritual dimension of the self as the prime determinant of health. Disability and Rehabilitation: An International, Multidisciplinary Journal, 28(11), 729-740. doi: 10.1080/09638280500265946

Ferreira, Manuela L., Machado, Gustavo, Latimer, Jane, Maher, Christopher, Ferreira, Paulo H., \& Smeets, Rob J. (2010a). Factors defining care-seeking in low back pain--A meta-analysis of population based surveys. European Journal of Pain, 14(7), e1-e7. doi: http://dx.doi.org/10.1016/j.ejpain.2009.11.005

Ferreira, Manuela L., Machado, Gustavo, Latimer, Jane, Maher, Christopher, Ferreira, Paulo H., \& Smeets, Rob J. (2010b). Factors defining care-seeking in low back pain-A meta-analysis of population based surveys. European Journal of Pain, 14(7), e1-e7. doi: http://dx.doi.org/10.1016/j.ejpain.2009.11.005

Feuerstein, Michael, Harrington, Cherise Baldwin, Lopez, Mary, \& Haufler, Amy. (2006). How Do Job Stress and Ergonomic Factors Impact Clinic Visits in Acute Low Back Pain? A Prospective Study. Journal of Occupational and Environmental Medicine, 48(6), 607-614. doi: http://dx.doi.org/10.1097/01.jom.0000205836.48069.aa

Fish, Rosemary A., Hogan, Michael J., Morrison, Todd G., Stewart, Ian, \& McGuire, Brian E. (2013). Willing and able: A closer look at Pain Willingness and Activity Engagement on the Chronic Pain Acceptance Questionnaire (CPAQ-8). The Journal of Pain, 14(3), 233-245. doi: dx.doi.org/10.1016/j.jpain.2012.11.004 
Fisher, Anne G. (2013). Occupation-centred, occupation-based, occupation-focused: Same, same or different? Scandinavian Journal of Occupational Therapy, 20(3), 162-173. doi: 10.3109/11038128.2012.754492

Fisher, Grace S., Emerson, Linda, Firpo, Camille, Ptak, Jan, Wonn, Jennifer, \& Bartolacci, Gwen. (2007). Chronic pain and occupation: An exploration of the lived experience. American Journal of Occupational Therapy, 61(3), 290-302. doi: http://dx.doi.org/10.5014/ajot.61.3.290

Flor, H., \& Turk, D. C. (2011). Chronic Pain: An integrated biobehavioral approach. Seattle: IASP Press.

Fluharty, G., Sellon, C., \& Glassman, N. (1994). Optimizing outcome through cognitive therapy and advocacy: a case study. Brain Injury, 8(8), 729-734. doi: dx.doi.org/10.3109/02699059409151027

Folkman, Susan, \& Lazarus, Richard S. (1980). An Analysis of Coping in a Middle-Aged Community Sample. Journal of Health and Social Behavior, 21(3), 219-239. doi: $10.2307 / 2136617$

Folkman, Susan, \& Moskowitz, Judith Tedlie. (2004). Coping: Pitfalls and Promise. Annual Review of Psychology, 55(1), 745-774. doi: doi:10.1146/annurev.psych.55.090902.141456

Fontanarosa, Phil B, \& Lundberg, George D. (1998). Alternative medicine meets science. Jama, 280(18), 1618-1619. doi: dx.doi.org/10.1001/jama.280.18.1618

Fordyce, W. E. (1974). Behavioral science and rehabilitation. Rehabilitation Psychology, 21(3), 82-85. doi: dx.doi.org/10.1037//0090-5550.21.3.82

Fordyce, W. E. (1976). Behavioral methods for chronic pain and illness. St. Louis: Mosby

Fordyce, W. E. (1981). Behavioral methods in medical rehabilitation. Neuroscience \& Biobehavioral Reviews, 5(3), 391-396. doi: dx.doi.org/10.1016/0149-7634(81)900336

Fordyce, W. E. (1982a). A behavioural perspective on chronic pain. British Journal of Clinical Psychology, 21(Pt 4), 313-320.

Fordyce, W. E. (1982b). A behavioural perspective on chronic pain. British Journal of Clinical Psychology, 21(4), 313-320. doi: dx.doi.org/10.1111/j.20448260.1982.tb00569.x

Fordyce, W. E. (1988). Pain and suffering: A reappraisal. American Psychologist, 43(4), 276283. doi: dx.doi.org/10.1037//0003-066X.43.4.276

Fordyce, W. E., Roberts, A. H., \& Sternbach, R. A. (1985). The behavioral management of chronic pain: a response to critics. Pain, 22(2), 113-125. doi: 10.1016/03043959(85)90172-1

Fordyce, Wilbert E., Fowler, Roy S., \& Delateur, Barbara. (1968). An Application of Behavior Modification Technique to a Problem of Chronic Pain. Behaviour Research and Therapy, 6(1), 105-107. doi: dx.doi.org/10.1016/0005-7967(68)90048-X 
Fordyce, Wilbert E., Shelton, John L., \& Dundore, Diana E. (1982). The modification of avoidance learning pain behaviors. Journal of Behavioral Medicine, 5(4), 405-414. doi: dx.doi.org/10.1007/BF00845370

Forsyth, Craig J., \& Simpson, Jessica. (2008). Everything Changes Once you Hang: Flesh Hook Suspension. Deviant Behavior, 29(4), 367-387. doi: 10.1080/01639620701588305

Fox, Eric J. (2006). Constructing a Pragmatic Science of Learning and Instruction with Functional Contextualism. Educational Technology, Research and Development, 54(1), 5-36. doi: 10.1002/pfi.4170280205

Frank, G., \& Polkinghorn, D. (2010). Qualitative research in occupational therapy: From the first to the second generation. Occupational Therapy Journal of Research: Occupation, Participation and Health, 30(2), 51 - 57. doi: 10.3928/1539449220100325-02

Frankl, Viktor E. (1959, 1962, 1984). Man's search for meaning. Boston, MA: Simon and Schuster.

Freitas, Antonio L., \& Higgins, E. (2002). Enjoying goal-directed action: The role of regulatory fit. Psychological Science, 13(1), 1-6. doi: http://dx.doi.org/10.1111/1467$\underline{9280.00401}$

Freitas, Antonio L., Liberman, Nira, \& Higgins, E. (2002). Regulatory fit and resisting temptation during goal pursuit. Journal of Experimental Social Psychology, 38(3), 291-298. doi: http://dx.doi.org/10.1006/jesp.2001.1504

Friedman, Elliot M., \& Ryff, Carol D. (2012). Living well with medical comorbidities: A biopsychosocial perspective. The Journals of Gerontology: Series B: Psychological Sciences and Social Sciences, 67B(5), 535-544. doi: dx.doi.org/10.1093/geronb/gbr152

Gatchel, Robert J. (2004). Comorbidity of chronic pain and mental health disorders: the biopsychosocial perspective. American Psychologist, 59(8), 795. doi: dx.doi.org/10.1037/0003-066X.59.8.795

Gatchel, Robert J. (2012). Editorial: New beginnings and the Institute of Medicine's Report on Living well with chronic illness: A call for public health action. Journal of Applied Biobehavioral Research, 17(1), 1-3. doi: dx.doi.org/10.1111/j.17519861.2012.00078.x

Gauthier, N., Thibault, P., \& Sullivan, M. J. (2008). Individual and relational correlates of pain-related empathic accuracy in spouses of chronic pain patients. Clinical Journal of Pain, 24(8), 669-677. doi: 10.1097/AJP.0b013e318173c28f

Giardino, N. D., Jensen, M. P., Turner, J. A., Ehde, D. M., \& Cardenas, D. D. (2003). Social environment moderates the association between catastrophizing and pain among persons with a spinal cord injury. Pain, 106(1-2), 19-25. doi: dx.doi.org/10.1016/S0304-3959(03)00226-4

Gilibert, Daniel, \& Banovic, Ingrid. (2009). Effect of training in psychology on the causal interpretation of a clinical case. European Journal of Psychology of Education, 24(3), 373-385. doi: http://dx.doi.org/10.1007/BF03174767 
Gillham, B. (2000). Case study methods. London/New York, NY: Bloomsbury Academic.

Gillon, R (1994). Medical ethics: four principles plus attention to scope. BMJ, 309(6948), 184. doi: $10.1136 / \mathrm{bmj} .309 .6948 .184$

Glaser, B. (1965). The constant comparative method of qualitative analysis. Social problems, $12(4), 436-445$.

Glaser, B. (1978). Theoretical sensitivity: Advances in the methodology of grounded theory (Vol. 2). Mill Valley, CA: Sociology Press

Glaser, B. (1998). Doing grounded theory: Issues and discussions. Mill Valley, CA: Sociology Press

Glaser, B. (2003). The grounded theory perspective II: Descriptions remodeling of grounded theory methodology. Mill Valley, CA.: Sociology Press.

Glaser, B. (2005). The grounded theory perspective III: Theoretical coding. Mill Valley, CA.: Sociology Press.

Glaser, B. (2011). Getting out of the Data: Grounded theory conceptualization. Mill Valley, California: Sociology Press.

Glaser, B G. (2008). Conceptualization: On theory and theorizing using grounded theory. International Journal of Qualitative Methods, 1(2), 23-38.

Glaser, B, \& Holton, J. (2007a). Remodeling grounded theory. Historical Social Research/Historische Sozialforschung. Supplement, 47-68.

Glaser, B, \& Strauss, A. (1967). The discovery of grounded theory: Strategies for qualitative research. New Jersey: AldineTransaction, A Division of Transaction Publishers, Rutgers.

Glaser, B., \& Holton, J. (2004). Remodeling grounded theory. Forum: Qualitative Social Research Sozialforschung, 5(2), 1-17.

Glaser, B., \& Strauss, A. (1965). Discovery of substantive theory: A basic strategy underlying qualitative research. American Behavioral Scientist, 8(6), 5-12. doi: dx.doi.org/10.1177/000276426500800602

Glaser, B.G. (1992). Emergence vs forcing: Basics of grounded theory analysis. Mill Valley, CA.: Sociology Press.

Glaser, B.G. (1995). Grounded Theory: 1984-1994. Mill Valley, CA.: Sociology Press.

Glaser, B.G. (2002). Constructivist grounded theory? Paper presented at the Forum Qualitative Sozialforschung/Forum: Qualitative Social Research.

Glaser, B.G., \& Holton, J. (2007b). Remodeling grounded theory. Historical Social Research/Historische Sozialforschung. Supplement, 47-68.

Glaser, Barney G. (1999). The Future of Grounded Theory. Qualitative Health Research, 9(6), 836-845. doi: 10.1177/104973299129122199

Glaser, Barney G. (2007). Constructivist grounded theory? . Forum: Qualitative Social Research Sozialforschung, 3(3), 66-75. 
Glucklich, Ariel. (1999). Self and sacrifice: A phenomenological psychology of sacred pain. Harvard Theological Review, 92(04), 479-506.

Goldberg, D. S., \& McGee, S. J. (2011). Pain as a global public health priority. BMC Public Health, 11, 770. doi: doi:10.1186/1471-2458-11-770

Goldberg, Richard W., Dickerson, Faith, Lucksted, Alicia, Brown, Clayton H., Weber, Elyssa, Tenhula, Wendy N., . . . Dixon, Lisa B. (2013). Living well: An intervention to improve self-management of medical illness for individuals with serious mental illness. Psychiatric Services, 64(1), 51-57. doi: dx.doi.org/10.1176/appi.ps.201200034

Goossens, Cees. (2000). Tourism information and pleasure motivation. Annals of Tourism Research, 27(2), 301-321. doi: dx.doi.org/10.1016/S0160-7383(99)00067-5

Goossens, M. E., Kindermans, H. P., Morley, S. J., Roelofs, J., Verbunt, J., \& Vlaeyen, J. W. (2010). Self-discrepancies in work-related upper extremity pain: relation to emotions and flexible-goal adjustment. European Journal of Pain, 14(7), 764-770. doi: dx.doi.org/10.1016/j.ejpain.2009.11.012

Goubert, L., Crombez, G., Eccleston, C., \& Devulder, J. (2004). Distraction from chronic pain during a pain-inducing activity is associated with greater post-activity pain. Pain, 110(1-2), 220-227. doi: dx.doi.org/10.1016/j.pain.2004.03.034

Goubert, Liesbet, Craig, K., Vervoort, Tine, Morley, S., Sullivan, M., Williams, A., . . . Crombez, G. (2005). Facing others in pain: The effects of empathy. Pain, 118(3), 285-288. doi: dx.doi.org/10.1016/j.pain.2005.10.025

Goubert, Liesbet, Crombez, Geert, \& De Bourdeaudhuij, Ilse. (2004). Low back pain, disability and back pain myths in a community sample: prevalence and interrelationships. European Journal of Pain, 8(4), 385-394. doi: 10.1016/j.ejpain.2003.11.004

Goubert, Liesbet, Vlaeyen, Johan W. S., Crombez, Geert, \& Craig, Kenneth D. (2011). Learning about pain from others: an observational learning account. Journal of Pain, 12(2), 167-174.

Grahn, B., Ekdahl, C., \& Borgquist, L. (2000). Motivation as a predictor of changes in quality of life and working ability in multidisciplinary rehabilitation: A two-year follow-up of a prospective controlled study in patients with prolonged musculoskeletal disorders. Disability \& Rehabilitation: An International Multidisciplinary Journal, 22(15), 639654.

Green, Carmen R., Baker, Tamara A., \& Ndao-Brumblay, S. (2004). Patient Attitudes Regarding Healthcare Utilization and Referral: A Descriptive Comparison in Africanand Caucasian Americans With Chronic Pain. Journal of the National Medical Association, 96(1), 31-42.

Greenwald, Anthony G, \& Krieger, Linda Hamilton. (2006). Implicit bias: Scientific foundations. California Law Review, 94(4), 945-967.

Grooten, Wilhelmus Johannes Andreas. (2007). Predictors for persistent neck/shoulder pain, medical care-seeking due to neck/shoulder pain and sickness absence. Clinical Rehabilitation, 21(7), 648-659. doi: http://dx.doi.org/10.1177/0269215507077263 
Gross, Douglas P. , Ferrari, Robert, Russell, Anthony S. , Battie, Michele C. , Schopflocher, Donald , Hu, Richard W. , . . Buchbinder, Rachelle (2006). A Population-Based Survey of Back Pain Beliefs in Canada. Spine, 31(8), 2142-2145. doi: 10.1097/01.brs.0000231771.14965.e4

Grotle, M., Brox, J., Glomsrod, B., Lonn, J., \& Vollestad, N. (2007). The association of socioeconomic status and psychosocial and physical workplace factors with musculoskeletal injury in hospital workers. American Journal of Industrial Medicine, 50(4), 245-260.

Guba, E.G., \& Lincoln, Y.S. (1994). Competing paradigms in qualitative research. In N. K. Denzin \& Y. S. Lincoln (Eds.), Handbook of qualitative research (pp. 105-118). Thousand Oaks, CA.: Sage.

Guite, Jessica W., Logan, Deirdre E., McCue, Rebecca, Sherry, David D., \& Rose, John B. (2009). Parental beliefs and worries regarding adolescent chronic pain. Clinical Journal of Pain, 25(3), 223-232. doi: dx.doi.org/10.1097/AJP.0b013e31818a7467

Gulbrandsen, Pal, Madsen, Henrik Bjarke, Benth, Jurate Saltyte, \& Laerum, Even. (2010). Health care providers communicate less well with patients with chronic low back pain-A study of encounters at a back pain clinic in Denmark. Pain, 150(3), 458-461. doi: http://dx.doi.org/10.1016/j.pain.2010.05.024

Gullacksen, A. C., \& Lidbeck, J. (2004). The life adjustment process in chronic pain: psychosocial assessment and clinical implications. Pain Research \& Management, 9(3), 145-153.

Gureje, Oye. (2007). Psychiatric aspects of pain. Current Opinion in Psychiatry, 20(1), 4246. doi: http://dx.doi.org/10.1097/YCO.0b013e328010ddf4

Hadjistavropoulos, H. D., MacLeod, F. K., \& Asmundson, G. J. (1999). Validation of the Chronic Pain Coping Inventory. Pain, 80(3), 471-481. doi: dx.doi.org/10.1016/S03043959(98)00224-3

Hadjistavropoulos, T., Craig, K. D., \& Fuchs-Lacelle, S. (2004). Social Influences and the Communication of Pain. In T. Hadjistavropoulos \& K. D. Craig (Eds.), Pain: Psychological perspectives (pp. 87-112). New Jersey, USA: Lawrence Erlbaum Associates, Inc.

Hadjistavropoulos, Thomas, Craig, Kenneth D., Duck, Steve, Cano, Annmarie, Goubert, Liesbet, Jackson, Philip L., . . Fitzgerald, Theresa Dever. (2011). A Biopsychosocial Formulation of Pain Communication. Psychological Bulletin, 137(6), 910-939. doi: dx.doi.org/10.1037/a0023876

Hadler, Nortin M, \& Carey, Timothy S. (1998). Low back pain: an intermittent and remittent predicament of life. Annals of the Rheumatic Diseases, 57(1), 1-2. doi: 10.1136/ard.57.1.1

Hagger, Martin S., \& Orbell, Sheina. (2003). A Meta-Analytic Review of the Common-Sense Model of Illness Representations. Psychology \& Health, 18(2), 141-184. doi: 10.1080/088704403100081321

Haig, B.D. (2008a). Scientific method, abduction, and clinical reasoning. Journal of Clinical Psychology, 64(9), 1013-1018. doi: dx.doi.org/10.1002/jclp.20505 
Haig, Brian D. (2008b). On the permissiveness of the abductive theory of method. Journal of Clinical Psychology, 64(9), 1037-1045. doi: 10.1002/jclp.20507

Haig, Brian D. (2008c). Scientific method, abduction, and clinical reasoning. doi: http://dx.doi.org/10.1002/jclp.20505

Hamilton, Nancy A., Karoly, Paul, \& Kitzman, Heather. (2004). Self-regulation and chronic pain: The role of emotion. Cognitive Therapy and Research, 28(5), 559-576. doi: http://dx.doi.org/10.1023/B:COTR.0000045565.88145.76

Han, S., Fan, Y., Xu, X., Qin, J., Wu, B., Wang, X., . . Mao, L. (2009). Empathic neural responses to others' pain are modulated by emotional contexts. Human Brain Mapping, 30(10), 3227-3237. doi: dx.doi.org/10.1002/jclp.20505

Handfield, Robert B., \& Melnyk, Steven A. (1998). The scientific theory-building process: a primer using the case of TQM. Journal of Operations Management, 16(4), 321-339. doi: http://dx.doi.org/10.1016/S0272-6963(98)00017-5

Hanley, E. N., Jr., Herkowitz, H. N., Kirkpatrick, J. S., Wang, J. C., Chen, M. N., \& Kang, J. D. (2010). Debating the value of spine surgery. Journal of Bone \& Joint Surgery American Volume, 92(5), 1293-1304.

Hanlon, R., Turk, D.., \& Rudy, T. E. (1987). A collaborative approach in the treatment of chronic pain. British Journal of Guidance \& Counselling, 15(1), 37-49. doi: http://dx.doi.org/10.1080/03069888700760051

Hansson, T., Hansson, E., \& Malchau, H. (2008). Utility of spine surgery: a comparison of common elective orthopaedic surgical procedures. Spine, 33(25), 2819-2830. doi: dx.doi.org/10.1097/BRS.0b013e31818e2914

Hardin, Erin E., \& Leong, Frederick T. L. (2005). Optimism and Pessimism as Mediators of the Relations Between Self-Discrepancies and Distress Among Asian and European Americans. Journal of Counseling Psychology, 52(1), 25-35. doi: dx.doi.org/10.1037/0022-0167.52.1.25

Harman, G.H. (1965). The inference to the best explanation. The Philosophical Review, 74(1), 88-95. doi: dx.doi.org/10.2307/2183532

Harper, Phil. (2006). No pain, no gain: pain behaviour in the armed forces. British Journal of Nursing, 15(10), 548-551. doi: dx.doi.org/10.12968/bjon.2006.15.10.21130

Harris, Samantha, Morley, Stephen, \& Barton, Stephen B. (2003). Role loss and emotional adjustment in chronic pain. Pain, 105(1-2), 363-370. doi: http://dx.doi.org/10.1016/S0304-3959\%2803\%2900251-3

Hartman, S. E., \& Norton, J. M. (2002). Craniosacral therapy is not medicine. Physical therapy, 82(11), 1146-1147.

Hasenbring, Monika I., Hallner, Dirk, \& Rusu, Adina C. (2009). Fear-avoidance- and endurance-related responses to pain: Development and validation of the AvoidanceEndurance Questionnaire (AEQ). doi: http://dx.doi.org/10.1016/j.ejpain.2008.11.001

Hassin, Ran R., Bargh, John A., \& Zimerman, Shira. (2009). Automatic and Flexible: The Case of Non-conscious Goal Pursuit. Social cognition, 27(1), 20-36. 
Hayes, S. C. , Luoma, J. B., Bond, F. W. , Masuda, A., \& Lillis, J. (2006). Acceptance and commitment therapy: Model, processes and outcomes. Behaviour Research \& Therapy, 44(1), 1-25. doi: dx.doi.org/10.1016/j.brat.2005.06.006

Hayes, S. C., Wilson, K. G., Gifford, E. V., Follette, V. M., \& Strosahl, K. D. (1996). Experiential Avoidance and Behavioral Disorders: A Functional Dimensional Approach to Diagnosis and Treatment. Journal of Consulting and Clinical Psychology, 64(6), 1152-1168. doi: dx.doi.org/10.1037//0022-006X.64.6.1152

Hayes, Steven C., \& Duckworth, Melanie P. (2006). Acceptance and Commitment Therapy and Traditional Cognitive Behavior Therapy Approaches to Pain. Cognitive and Behavioral Practice, 13(3), 185-187. doi: http://dx.doi.org/10.1016/j.cbpra.2006.04.002

Haythornthwaite, Jennifer A., \& Heinberg, Leslie J. (1999). Coping with pain: What works, under what circumstances, and in what ways? Pain Forum, 8(4), 172-175. doi: http://dx.doi.org/10.1016/S1082-3174(99)70003-6

Heath, Helen. (2006). Exploring the influences and use of the literature during a grounded theory study. Journal of Research in Nursing, 11(6), 519-528. doi: $10.1177 / 1744987106069338$

Heath, Helen, \& Cowley, Sarah. (2004). Developing a grounded theory approach: A comparison of Glaser and Strauss. International Journal of Nursing Studies, 41(2), 141-150. doi: http://dx.doi.org/10.1016/S0020-7489\%2803\%2900113-5

Hegelund, A. (2005). Objectivity and Subjectivity in the Ethnographic Method. Qualitative Health Research, 15(15), 647-668. doi: 10.1177/1049732304273933

Heim, E, Valach, L, \& Schaffner, L. (1997). Coping and psychosocial adaptation: longitudinal effects over time and stages in breast cancer. Psychosomatic Medicine, 59(4), 408-418. doi: dx.doi.org/10.1097/00006842-199707000-00011

Hellstrom, Christina. (2001). Temporal dimensions of the self-concept: Entrapped and possible selves in chronic pain. Psychology \& Health, 16(1), 111-124. doi: http://dx.doi.org/10.1080/08870440108405493

Hellstrom, Christina, \& Carlsson, Sven G. (1996). The long-lasting now: Disorganization in subjective time in long-standing pain. Scandinavian Journal of Psychology, 37(4), 416-423. doi: 10.1111/j.1467-9450.1996.tb00673.x

Henwood, K, \& Pidgeon, N. (2003). Grounded theory in psychological research. In P. M. R. Camic, Jean E; Yardley, Lucy (Ed.), Qualitative research in psychology: Expanding perspectives in methodology and design (pp. 131-155). Washington, DC: American Psychological Association.

Henwood, K, \& Pidgeon, N. (2006). Grounded theory. In G. M. Breakwell, S. Hammond, C. Fife-Shaw \& J. A. Smith (Eds.), Research methods in psychology (3 ed.). Thousand Oaks, CA: Sage.

Henwood, K.L., \& Pidgeon, N.F. (1992). Qualitative research and psychological theorizing. British Journal of Psychology, 83(1), 97-111. doi: dx.doi.org/10.1111/j.20448295.1992.tb02426.x 
Henwood, Penelope, Ellis, Jacqueline, Logan, Jo, Dubouloz, Claire-Jehanne, \& D'Eon, Joyce. (2012). Acceptance of Chronic Neuropathic Pain in Spinal Cord Injured Persons: A Qualitative Approach. Pain management nursing : official journal of the American Society of Pain Management Nurses, 13(4), 215-222. doi: dx.doi.org/10.1016/j.pmn.2010.05.005

Herrman, Helen, Stewart, Donna E., Diaz-Granados, Natalia, Berger, Elena L., Jackson, Beth, \& Yuen, Tracy. (2011). What is resilience? The Canadian Journal of Psychiatry / La Revue canadienne de psychiatrie, 56(5), 258-265.

Higgins, E Tory. (1987). Self-discrepancy: a theory relating self and affect. Psychological review, 94(3), 319. doi: dx.doi.org/10.1037//0033-295X.94.3.319

Higgins, E. (1997). Beyond pleasure and pain. American Psychologist, 52(12), 1280-1300. doi: http://dx.doi.org/10.1037/0003-066X.52.12.1280

Higgins, E., Klein, Ruth L., \& Strauman, Timothy J. (1987). Self-discrepancies: Distinguishing among self-states, self-state conflicts, and emotional vulnerabilities. Yardley, Krysia [Ed]; Honess, Terry [Ed] (1987) Self and identity: Psychosocial perspectives (pp 173-186) xvii, 332 pp Oxford, England: John Wiley \& Sons; England, 173-186.

Hinrichs-Rocker, Anke, Schulz, Kerstin, Jarvinen, Imke, Lefering, Rolf, Simanski, Christian, \& Neugebauer, Edmund A. (2009). Psychosocial predictors and correlates for chronic post-surgical pain (CPSP) - A systematic review. European Journal of Pain, 13(7), 719-730. doi: dx.doi.org/10.1016/j.ejpain.2008.07.015

Hitchcock, C., \& Sober, E. (2004). Prediction versus accommodation and the risk of overfitting. The British journal for the philosophy of science, 55(1), 1-34. doi: dx.doi.org/10.1093/bjps/55.1.1

Hoare, Karen J., Mills, Jane, \& Francis, Karen. (2012). Dancing with data: An example of acquiring theoretical sensitivity in a grounded theory study. International Journal of Nursing Practice, 18(3), 240-245. doi: 10.1111/j.1440-172X.2012.02038.x

Hobara, Mieko. (2005). Beliefs about appropriate pain behavior: Cross-cultural and sex differences between Japanese and Euro-Americans. European Journal of Pain, 9(4), 389-393. doi: http://dx.doi.org/10.1016/j.ejpain.2004.09.006

Hocking, C. (2009). The challenge of occupation: describing the things people do. Journal of Occupational Science, 16(3), 140-150. doi:

dx.doi.org/10.1080/14427591.2009.9686655

Holt, Nicholas L., \& Tamminen, Katherine A. (2010). Moving forward with grounded theory in sport and exercise psychology. Psychology of Sport and Exercise, 11(6), 419-422. doi: 10.1016/j.psychsport.2010.07.009

Holton, Judith. (2010). The Coding Process and Its Challenges | Grounded Theory. Grounded Theory Review: An international journal, 9(1), 21-40.

Holzl, Rupert, Kleinbohl, Dieter, \& Huse, Ellena. (2005). Implicit operant learning of pain sensitization. Pain, 115(1-2), 12-20. doi: dx.doi.org/10.1016/j.pain.2005.01.026 
Horton, Alicia D. (2012). Flesh Hook Pulling: Motivations and Meaning-Making From the "Body Side" of Life. Deviant Behavior, 34(2), 115-134. doi: 10.1080/01639625.2012.707547

House, Ernest R. (1991). Realism in Research. Educational Researcher, 20(6), 2-25. doi: $10.2307 / 1176898$

Howell, Suzanne L. (1994). A theoretical model for caring for women with chronic nonmalignant pain. Qualitative Health Research, 4(1), 94-122. doi: http://dx.doi.org/10.1177/104973239400400107

Hsieh, A. Y., Tripp, D. A., \& Ji, L. J. (2011). The influence of ethnic concordance and discordance on verbal reports and nonverbal behaviours of pain. Pain, 152(9), 20162022. doi: dx.doi.org/10.1016/j.pain.2011.04.023

Huehls, F. (2005). An Evening of Grounded Theory: Teaching Process through Demonstration and Simulation. The Qualitative Report, 10(2), 328-338.

Huggins, Jennifer L., Bonn-Miller, Marcel O., Oser, Megan L., Sorrell, John T., \& Trafton, Jodie A. (2012). Pain anxiety, acceptance, and outcomes among individuals with HIV and chronic pain: A preliminary investigation. Behaviour Research and Therapy, 50(1), 72-78. doi: dx.doi.org/10.1016/j.brat.2011.10.008

Huijnen, Ivan P., Verbunt, Jeanine A., Peters, Madelon L., Delespaul, Philippe, Kindermans, Hanne P., Roelofs, Jeffrey, . . . Seelen, Henk A. (2010). Do depression and pain intensity interfere with physical activity in daily life in patients with chronic low back pain? Pain, 150(1), 161-166. doi: http://dx.doi.org/10.1016/j.pain.2010.04.021

Hunt, M, Chan, L., \& Mehta, A. (2011). Transitioning from Clinical to Qualitative Research Interviewing. International Journal of Qualitative Methods, 10(3), 191-201.

Huot, S., \& Rudman, D. L. (2010). The performances and places of identity: conceptualizing intersections of occupation, identity and place in the process of migration. Journal of Occupational Science, 17(2), 68-77. doi: dx.doi.org/10.1080/14427591.2010.9686677

Ikiugu, M, Pollard, N, Cross, A, Willer, M, Everson, J, \& Stockland, J. (2012). Meaning making through occupations and occupational roles: A heuristic study of workerwriter histories. British Journal of Occupational Therapy, 75(6), 289-295. doi: dx.doi.org/10.1080/14427591.2010.9686677

Irvine, H., Davidson, C., Hoy, K., \& Lowe-Strong, A. (2009). Psychosocial adjustment to multiple sclerosis: exploration of identity redefinition. Disability \& Rehabilitation, 31(8), 599-606. doi: 10.1080/09638280802243286

Jacob, Gary. (2003). Biopsychosocial perspective on low back pain: patient provider communications. Journal of Minimally Invasive Spinal Techniques, 3, 27 - 35.

Jacob, T., Zeev, A., \& Epstein, L. (2003). Low back pain--a community-based study of careseeking and therapeutic effectiveness. Disability \& Rehabilitation, 25(2), 67-76. doi: dx.doi.org/10.1080/0963828021000007905

Jeffe, Molly Moore, Butler, Mary, Stark, Alice, \& Kane, Robert L. (2011). Multidisciplinary Pain Programs for Chronic Noncancer Pain. Rockville, MD.: Agency for Healthcare Research and Quality. 
Jensen, Jens Christian, Haahr, Jens Peder, Frost, Poul, \& Andersen, Johan Hviid. (2012). The significance of health anxiety and somatization in care-seeking for back and upper extremity pain. Family Practice, 29(1), 86-95. doi: http://dx.doi.org/10.1093/fampra/cmr046

Jensen, M. P., Turner, J. A., Romano, J. M., \& Strom, S. E. (1995). The Chronic Pain Coping Inventory: development and preliminary validation. Pain, 60(2), 203-216. doi: dx.doi.org/10.1016/0304-3959(94)00118-X

Jensen, Mark P., Turner, Judith A., Romano, Joan M., \& Karoly, Paul. (1991). Coping with chronic pain: A critical review of the literature. Pain, 47(3), 249-283. doi: http://dx.doi.org/10.1016/0304-3959\%2891\%2990216-K

Jeon, Yun-Hee. (2004). The application of grounded theory and symbolic interactionism. Scandinavian Journal of Caring Sciences, 18(3), 249-256. doi: 10.1111/j.14716712.2004.00287.x

Johnson Wright, Lisa, Afari, Niloofar, \& Zautra, Alex. (2009). The illness uncertainty concept: a review. Current Pain \& Headache Reports, 13(2), 133-138. doi: dx.doi.org/10.1007/s11916-009-0023-z

Jones, J. (2009). Selection of Grounded Theory as an Appropriate Research Methodology for a Dissertation: One Student's Perspective. . The Grounded Theory Review, 8(2), 23 34.

Josephson, John R, \& Josephson, Susan G. (1996). Abductive inference: Computation, philosophy, technology. Cambridge: Cambridge University Press.

Jull, G. A., Soderlund, A., Stemper, B. D., Kenardy, J., Gross, A. R., Cote, P., . . Curatolo, M. (2011). Toward optimal early management after whiplash injury to lessen the rate of transition to chronicity: discussion paper 5. Spine, 36(25 Suppl), S335-342. doi: dx.doi.org/10.1097/BRS.0b013e3182388449

Kabat-Zinn, Jon. (1982). An outpatient program in behavioral medicine for chronic pain patients based on the practice of mindfulness meditation: Theoretical considerations and preliminary results. General hospital psychiatry, 4(1), 33-47. doi: http://dx.doi.org/10.1016/0163-8343(82)90026-3

Kantartzis, Sarah, \& Molineux, Matthew. (2011). The Influence of Western Society's Construction of a Healthy Daily Life on the Conceptualisation of Occupation. Journal of Occupational Science, 18(1), 62-80. doi: http://dx.doi.org/10.1080/14427591.2011.566917

Karenberg, A., \& Leitz, C. (2001). Headache in magical and medical papyri of Ancient Egypt. Cephalalgia, 21(9), 911-916. doi: 10.1046/j.1468-2982.2001.00274.x

Karoly, P., \& Ruehlman, L. S. (2006). Psychological "resilience" and its correlates in chronic pain: findings from a national community sample. Pain, 123(1-2), 90-97. doi: dx.doi.org/10.1016/j.pain.2006.02.014

Karoly, Paul, Okun, Morris A., Enders, Craig, \& Tennen, Howard. (2014). Effects of Pain Intensity on Goal Schemas and Goal Pursuit: A Daily Diary Study. Health Psychology, 33(9), 968-976. doi: dx.doi.org/10.1037/hea0000093 
Karoly, Paul, Ruehlman, Linda S., Aiken, Leona S., Todd, Michael, \& Newton, Craig. (2006). Evaluating Chronic Pain Impact among Patients in Primary Care: Further Validation of a Brief Assessment Instrument. Pain Medicine, 7(4), 289-298. doi: http://dx.doi.org/10.1111/j.1526-4637.2006.00182.x

Karsdorp, P. A., \& Vlaeyen, J. W. (2009). Active avoidance but not activity pacing is associated with disability in fibromyalgia. Pain, 147(1-3), 29-35. doi: dx.doi.org/10.1016/j.pain.2009.07.019

Kearney, Margaret H. (2009). Taking grounded theory beyond psychological process. Research in Nursing \& Health, 32(6), 567-568. doi: 10.1002/nur.20345

Keefe, F. J., Rumble, M. E., Scipio, C. D., Giordano, L. A., \& Perri, L. M. (2004). Psychological aspects of persistent pain: current state of the science. Journal of Pain, 5(4), 195-211. doi: dx.doi.org/10.1016/j.jpain.2004.02.576

Keefe, Francis J., Dunsmore, Julie, \& Burnett, Rachel. (1992). Behavioral and CognitiveBehavioral Approaches to Chronic Pain: Recent Advances and Future Directions. Journal of Consulting \& Clinical Psychology, 60(4), 528-536. doi: dx.doi.org/10.1037//0022-006X.60.4.528

Keefe, Francis J., Salley, Alfred N., \& Lefebvre, John C. (1992). Coping with pain: Conceptual concerns and future directions. Pain, 51(2), 131-134. doi: http://dx.doi.org/10.1016/0304-3959\%2892\%2990253-8

Kelle, Udo. (2007a). The Development of Categories: Different Approaches in Grounded Theory. The SAGE Handbook of Grounded Theory. SAGE Publications Ltd. In A. Bryant \& K. Charmaz (Eds.), The SAGE Handbook of Grounded Theory (pp. 191214). London, England: SAGE Publications Ltd.

Kelle, Udo. (2007b). " Emergence" vs." Forcing" of Empirical Data? A Crucial Problem of" Grounded Theory" Reconsidered. Paper presented at the Historical Social Research/Historische Sozialforschung. Supplement.

Kelly, George. (1955). The psychology of personal constructs (1 ed.). New York: Norton.

Kelly, M, Lamont, S, \& Brunero, S. (2010). An occupational perspective of the recovery journey in mental health. British Journal of Occupational Therapy, 73(3), 129-135.

Kikuchi, H., Yoshiuchi, K., Miyasaka, N., Ohashi, K., Yamamoto, Y., Kumano, H., . . Akabayashi, A. (2006). Reliability of recalled self-report on headache intensity: Investigation using ecological momentary assessment technique. Cephalalgia, 26(11), 1335-1343. doi: dx.doi.org/10.1111/j.1468-2982.2006.01221.x

Kindermans, Hanne P., Goossens, Marielle E., Roelofs, Jeffrey, Huijnen, Ivan P., Verbunt, Jeanine A., Morley, Stephen, \& Vlaeyen, Johan W. (2010). A content analysis of ideal, ought, and feared selves in patients with chronic low back pain. European Journal of Pain, 14(6), 648-653. doi: http://dx.doi.org/10.1016/j.ejpain.2009.10.012

Kindermans, Hanne P., Huijnen, Ivan P., Goossens, Marielle E., Roelofs, Jeffrey, Verbunt, Jeanine A., \& Vlaeyen, Johan W. (2011). "Being" in pain: The role of selfdiscrepancies in the emotional experience and activity patterns of patients with chronic low back pain. Pain, 152(2), 403-409. doi: http://dx.doi.org/10.1016/j.pain.2010.11.009 
Kindermans, Hanne P., Roelofs, Jeffrey, Goossens, Marielle E., Huijnen, Ivan P., Verbunt, Jeanine A., \& Vlaeyen, Johan W. (2011). Activity patterns in chronic pain:

Underlying dimensions and associations with disability and depressed mood. The Journal of Pain, 12(10), 1049-1058. doi: http://dx.doi.org/10.1016/j.jpain.2011.04.009

Kirmayer, Laurence J. (2008). Culture and the metaphoric mediation of pain. Transcultural Psychiatry, 45(2), 318-338. doi: http://dx.doi.org/10.1177/1363461508089769

Kleiber, D. A., Reel, H. A., \& Hutchinson, S. L. (2008). When distress gives way to possibility: the relevance of leisure in adjustment to disability. NeuroRehabilitation, 23(4), 321-328.

Koch, Christof. (2004). The quest for consciousness. Englewood, Colorado, USA: New York.

Kraaimaat, Floris W., \& Evers, Andrea W. (2003). Pain-coping strategies in chronic pain patients: Psychometric characteristics of the pain-coping inventory (PCI). International Journal of Behavioral Medicine, 10(4), 343-363. doi: dx.doi.org/10.1207/S15327558IJBM1004_5

Kranz, Dirk, Bollinger, Annicka, \& Nilges, Paul. (2010). Chronic pain acceptance and affective well-being: A coping perspective. European Journal of Pain, 14(10), 10211025. doi: dx.doi.org/10.1016/j.ejpain.2010.03.010

Kratz, Anna L., Hirsh, Adam T., Ehde, Dawn M., \& Jensen, Mark P. (2013). Acceptance of pain in neurological disorders: Associations with functioning and psychosocial wellbeing. Rehabilitation Psychology, 58(1), 1-9. doi: dx.doi.org/10.1037/a0031727

Kress, Jeffrey L., \& Statler, Traci. (2007). A naturalistic investigation of former Olympic cyclists' cognitive strategies for coping with exertion pain during performance. Journal of Sport Behavior, 30(4), 428+.

Krohne, H.W. (1996). Individual differences in coping. In M. Zeidner \& N. S. Endler (Eds.), Handbook of Coping: Theory, research, applications (pp. 381-409). New York: John Wiley \& Sons.

Kuhn, T.S., \& Hacking, I. (2012). The Structure of Scientific Revolutions: 50th Anniversary Edition: University of Chicago Press.

Kuhn, Thomas S. (2012). The structure of scientific revolutions (4 ed.). Chicago: University of Chicago press.

Kulich, R., \& Loeser, J. D. (2011). The business of pain medicine: the present mirrors antiquity. Pain Medicine, 12(7), 1063-1075. doi: 10.1111/j.1526-4637.2011.01176.x

Kuo, A. (2011). A transactional view: Occupation as a means to create experiences that matter. Journal of Occupational Science: Australia, 18(2), 131-138. doi: $10.1080 / 14427591.2011 .575759$

Labour, Department of. (2008). Workforce 2020 Forces for Change in the Future Labour Market of New Zealand. Retrieved from Ministry of Business, Innovation \& Employment website: http://www.dol.govt.nz/publications/research/forces-forchange/forces-for-change-06.asp 
Labus, Jennifer S., Keefe, Francis J., \& Jensen, Mark P. (2003). Self-reports of pain intensity and direct observations of pain behavior: when are they correlated? Pain, 102(1-2), 109-124. doi: http://dx.doi.org/10.1016/s0304-3959(02)00354-8

Lachapelle, D. L., Lavoie, S., \& Boudreau, A. (2008). The meaning and process of pain acceptance. Perceptions of women living with arthritis and fibromyalgia. Pain Research \& Management, 13(3), 201-210.

Lackner, J. M., \& Gurtman, M. B. (2004). Pain catastrophizing and interpersonal problems: a circumplex analysis of the communal coping model. Pain, 110(3), 597-604. doi: 10.1016/j.pain.2004.04.011

Large, Robert, \& Strong, Jenny. (1997). The personal constructs of coping with chronic low back pain: is coping a necessary evil? Pain, 73(2), 245-252. doi: http://dx.doi.org/10.1016/S0304-3959(97)00100-0

Larsen, D. K., Taylor, S., \& Asmundson, G. J. (1997). Exploratory factor analysis of the Pain Anxiety Symptoms Scale in patients with chronic pain complaints. Pain, 69(1-2), 2734. doi: dx.doi.org/10.1016/S0304-3959(96)03232-0

Larson, E., \& von Eye, A. (2010). Beyond flow: temporality and participation in everyday activities. American Journal of Occupational Therapy, 64(1), 152-163. doi: dx.doi.org/10.5014/ajot.64.1.152

Latham, P. M. (1862). General Remarks on the Practice of Medicine (Vol. 1).

Latremoliere, A., \& Woolf, C. J. (2009). Central sensitization: a generator of pain hypersensitivity by central neural plasticity. Journal of Pain, 10(9), 895-926.

Lauwerier, Emelien, Damme, Stefaan, Goubert, Liesbet, Paemeleire, Koen, Devulder, Jacques, \& Crombez, Geert. (2012). To control or not? A motivational perspective on coping with pain. Acta Neurologica Belgica, 112(1), 3-7. doi: 10.1007/s13760-0120020-6

Law, M. (2002). Participation in the occupations of everyday life. American Journal of Occupational Therapy, 56(6), 640-649. doi: dx.doi.org/10.5014/ajot.56.6.640

Law, Mary, Baptiste, Sue, McColl, M, Opzoomer, Anne, Polatajko, Helene, \& Pollock, Nancy. (1990). The Canadian occupational performance measure: an outcome measure for occupational therapy. Canadian Journal of Occupational Therapy Revue Canadienne d Ergotherapie, 57(2), 82-87.

Lazarus, R. , \& Folkman, S. . (1984a). Stress, Appraisal, and Coping. New York: Springer.

Lazarus, R.S., \& Folkman, S. (1984b). Stress, appraisal, and coping. New York, NY: Springer Publishing Company.

Leadley, R. M., Armstrong, N., Lee, Y. C., Allen, A., \& Kleijnen, J. (2012). Chronic diseases in the European Union: the prevalence and health cost implications of chronic pain. Journal of Pain \& Palliative Care Pharmacotherapy, 26(4), 310-325. doi: dx.doi.org/10.3109/15360288.2012.736933

Leap, Nicky, \& Anderson, T. (2004). The role of pain in normal birth and the empowerment of women. In S. Downe (Ed.), Normal Childbirth: Evidence and debate (Vol. 1, pp. 25-39). London, UK: Churchill Livingstone. 
Lee Davis, D., \& Walker, K. (2011). Case-loading midwifery in New Zealand: bridging the normal/abnormal divide 'with woman'. Midwifery, 27(1), 46-52. doi: dx.doi.org/10.1016/j.midw.2009.09.007

Legrain, V., Damme, S. V., Eccleston, C., Davis, K. D., Seminowicz, D. A., \& Crombez, G. (2009). A neurocognitive model of attention to pain: behavioral and neuroimaging evidence. Pain, 144(3), 230-232. doi: dx.doi.org/10.1016/j.pain.2009.03.020

Lenhart, R., \& Ashby, Jeffrey S. (1996). Cognitive coping strategies and coping modes in relation to chronic pain disability. Journal of Applied Rehabilitation Counseling, 27(4), 15-18.

Lethem, J., Slade, P. D., Troup, J. D. G., \& Bentley, G. (1983). Outline of a fear-avoidance model of exaggerated pain perception. Behaviour Research and Therapy, 21(4), 401408. doi: http://dx.doi.org/10.1016/0005-7967(83)90009-8

Leventhal, Howard. (1970). Findings and theory in the study of fear communications. Advances in experimental social psychology, 5, 119-186. doi: dx.doi.org/10.1016/S0065-2601(08)60091-X

Leventhal, Howard, Diefenbach, Michael, \& Leventhal, Elaine A. (1992). Illness cognition: using common sense to understand treatment adherence and affect cognition interactions. Cognitive therapy and research, 16(2), 143-163. doi: dx.doi.org/10.1007/BF01173486

Lierh, P. (2005). Looking at symptoms with a middle-range theory lens. Advanced Studies in Nursing, 3(5), 152-157.

Livneh, Hanoch, \& Martz, Erin. (2007). An introduction to coping theory and research. In E. Martz \& H. Livneh (Eds.), Coping with chronic illness and disability: Theoretical, empirical, and clinical aspects (pp. 3-27). New York: Springer Science + Business Media.

Locke, Edwin A. (2007). The case for inductive theory building. Journal of Management, 33(6), 867-890. doi: http://dx.doi.org/10.1177/0149206307307636

Loeser, J. D., \& Melzack, R. (1999). Pain: an overview. Lancet, 353(9164), 1607-1609.

Loeser, JD. (1982). Concepts of pain. In M. Stanton-Hicks \& R. Boas (Eds.), Chronic low back pain (pp. 145-148). New York: Raven Press.

Loeser, JD. (2012). Five crises in pain management. Revista de la Sociedad Española del Dolor, 19(3), 111-116.

Loeser, John D. (2005a). Pain: Disease or Dis-ease? The John Bonica Lecture: Presented at the Third World Congress of World Institute of Pain, Barcelona 2004. Pain Practice, 5(2), 77-84. doi: 10.1111/j.1533-2500.2005.05204.x

Loeser, John D. (2005b). Quo Vadis, Poena. Journal of Musculoskeletal Pain, 13(3), 3-9. doi: dx.doi.org/10.1300/J094v13n03_02

Loeser, John D. (2006). Comprehensive Pain Programs Versus Other Treatments for Chronic Pain. The Journal of Pain 7(11), 800-801. doi: dx.doi.org/10.1016/j.jpain.2006.09.008 
Lomborg, Kirsten, \& Kirkevold, Marit. (2003). Truth and validity in grounded theory - a reconsidered realist interpretation of the criteria: fit, work, relevance and modifiability. Nursing Philosophy, 4(3), 189-200. doi: 10.1046/j.1466769X.2003.00139.x

Lunt, Alan. (2004). The implications for the clinician of adopting a recovery model: The role of choice in assertive treatment. Psychiatric Rehabilitation Journal, 28(1), 93-97. doi: dx.doi.org/10.2975/28.2004.93.97

Lynham, Susan A. (2002). The General Method of Theory-Building Research in Applied Disciplines. Advances in Developing Human Resources, 4(3), 221-241. doi: $10.1177 / 1523422302043002$

Macfarlane, G., Jones, G., \& Hannaford, P. (2006). How do job stress and ergonomic factors impact clinic visits in acute low back pain? A prospective study. Journal of Occupational and Environmental Medicine 48(6), 607-614.

Machin, Peggy. (1998). Stiff upper lip: Coping strategies of World War II veterans with phantom limb pain. The Clinical Journal of Pain, 14(4), 290-294. doi: dx.doi.org/10.1097/00002508-199812000-00004

MacKenzie, E. J., Bosse, M. J., Kellam, J. F., Pollak, A. N., Webb, L. X., Swiontkowski, M. F., . . Castillo, R. C. (2006). Early predictors of long-term work disability after major limb trauma. Journal of Trauma Injury Infection \& Critical Care, 61(3), 688-694. doi: dx.doi.org/10.1097/01.ta.0000195985.56153.68

MacLure, Maggie. (2010). The offence of theory. Journal of Education Policy, 25(2), 277286. doi: 10.1080/02680930903462316

Magnus, E. (2001). Everyday occupations and the process of redefinition: a study of how meaning in occupation influences redefinition of identity in women with a disability. Scandinavian Journal of Occupational Therapy, 8, 115-124. doi: dx.doi.org/10.1080/110381201750464467

Mailis-Cagnon, Angela, Yegneswaran, Balaji, Nicholson, Keith, Lakha, S., Papagapiou, Marios, Steiman, Amanda J., . . . Zurowski, Mateusz. (2007). Ethnocultural and sex characteristics of patients attending a tertiary care pain clinic in Toronto, Ontario. Pain Research \& Management, 12(2), 100-106.

Main, C J, Sullivan, M J L, \& Watson, P. J. (2008). Pain Management: practical applications of the biopsychosocial perspective (2 ed.). Edinburgh: Churchill Livingstone.

Main, C. J., Foster, N., \& Buchbinder, R. (2010). How important are back pain beliefs and expectations for satisfactory recovery from back pain? Best Practice \& Research Clinical Rheumatology, 24(2), 205-217. doi: doi:10.1016/j.berh.2009.12.012

Maly, Monica R., \& Krupa, Terry. (2007). Personal experience of living with knee osteoarthritis among older adults. Disability and Rehabilitation: An International, Multidisciplinary Journal, 29(18), 1423-1433. doi: 10.1080/09638280601029985

Manchikanti, L., Pampati, V., Singh, V., \& Falco, F. J. (2013). Assessment of the escalating growth of facet joint interventions in the medicare population in the United States from 2000 to 2011. Pain Physician, 16(4), E365-378. 
Manchikanti, Laxmaiah, Singh, Vijay, Falco, Frank J. E., Cash, Kimberly A., \& Pampati, Vidyasagar. (2008). Lumbar facet joint nerve blocks in managing chronic facet joint pain: one-year follow-up of a randomized, double-blind controlled trial: Clinical Trial NCT00355914. Pain Physician, 11(2), 121-132.

Manicas, Peter T., \& Secord, Paul F. (1983). Implications for psychology of the new philosophy of science. American Psychologist, 38(4), 399-413. doi: dx.doi.org/10.1037//0003-066X.38.4.399

Marhold, Charlotta, Linton, Steven J., \& Melin, Lennart. (2002). Identification of obstacles for chronic pain patients to return to work: Evaluation of a questionnaire. Journal of Occupational Rehabilitation, 12(2), 65-75.

Markus, H, \& Wurf, E. (1987). The Dynamic Self-Concept: A Social Psychological Perspective. Annual Review of Psychology, 38(1), 299-337. doi: doi:10.1146/annurev.ps.38.020187.001503

Markus, Hazel, \& Nurius, Paula. (1986). Possible Selves. American Psychologist, 41(9), $954-$ 969. doi: dx.doi.org/10.1037//0003-066X.41.9.954

Martel, M., Thibault, P., \& Sullivan, M. (2010). The persistence of pain behaviors in patients with chronic back pain is independent of pain and psychological factors. Pain, 151(2), 330-336. doi: http://dx.doi.org/10.1016/j.pain.2010.07.004

Mattson-Prince, J. (1997). A rational approach to long-term care: comparing the independent living model with agency-based care for persons with high spinal cord injuries. Spinal Cord, 35(5), 326-331. doi: dx.doi.org/10.1038/sj.sc.3100453

McCracken, L. M., \& Gutierrez-Martinez, O. (2011). Processes of change in psychological flexibility in an interdisciplinary group-based treatment for chronic pain based on Acceptance and Commitment Therapy. Behaviour Research \& Therapy, 49(4), 267274. doi: dx.doi.org/10.1016/j.brat.2011.02.004

McCracken, L. M., Vowles, K. E., \& Gauntlett-Gilbert, J. (2007). A prospective investigation of acceptance and control-oriented coping with chronic pain. Journal of Behavioral Medicine, 30(4), 339-349. doi: dx.doi.org/10.1007/s10865-007-9104-9

McCracken, L. M., \& Zhao-O'Brien, J. (2010). General psychological acceptance and chronic pain: there is more to accept than the pain itself. European Journal of Pain, 14(2), 170-175. doi: dx.doi.org/10.1016/j.ejpain.2009.03.004

McCracken, Lance M. (1998). Learning to live with the pain: acceptance of pain predicts adjustment in persons with chronic pain. Pain, 74(1), 21-27. doi: 10.1016/s03043959(97)00146-2

McCracken, Lance M. (2005). Social context and acceptance of chronic pain: The role of solicitous and punishing responses. Pain, 113(1-2), 155-159. doi: dx.doi.org/10.1016/j.pain.2004.10.004

McCracken, Lance M. (2010). Toward understanding acceptance and psychological flexibility in chronic pain. Pain, 149(3), 420-421. doi: http://dx.doi.org/10.1016/j.pain.2010.02.036 
McCracken, Lance M., \& Eccleston, Christopher. (2005). A prospective study of acceptance of pain and patient functioning with chronic pain. Pain, 118(1-2), 164-169. doi: dx.doi.org/10.1016/j.pain.2005.08.015

McCracken, Lance M., \& Eccleston, Christopher. (2006). A comparison of the relative utility of coping and acceptance-based measures in a sample of chronic pain sufferers. European Journal of Pain, 10(1), 23-29. doi: http://dx.doi.org/10.1016/j.ejpain.2005.01.004

McCracken, Lance M., \& Gauntlett-Gilbert, Jeremy. (2011). Role of psychological flexibility in parents of adolescents with chronic pain: Development of a measure and preliminary correlation analyses. Pain, 152(4), 780-785. doi: dx.doi.org/10.1016/j.pain.2010.12.001

McCracken, Lance M., Gauntlett-Gilbert, Jeremy, \& Vowles, Kevin E. (2007). The role of mindfulness in a contextual cognitive-behavioral analysis of chronic pain-related suffering and disability. Pain, 131(1-2), 63-69. doi: http://dx.doi.org/10.1016/j.pain.2006.12.013

McCracken, Lance M., Hoskins, Julian, \& Eccleston, Christopher. (2006). Concerns About Medication and Medication Use in Chronic Pain. Journal of Pain, 7(10), 726-734. doi: dx.doi.org/10.1016/j.jpain.2006.02.014

McCracken, Lance M., \& Jones, Rosie. (2012). Treatment for chronic pain for adults in the seventh and eighth decades of life: A preliminary study of acceptance and commitment therapy (ACT). Pain Medicine, 13(7), 860-867. doi: dx.doi.org/10.1111/j.1526-4637.2012.01407.x

McCracken, Lance M., MacKichan, Fiona, \& Eccleston, Christopher. (2007). Contextual cognitive-behavioral therapy for severely disabled chronic pain sufferers: Effectiveness and clinically significant change. European Journal of Pain, 11(3), 314322. doi: dx.doi.org/10.1016/j.ejpain.2006.05.004

McCracken, Lance M., \& Morley, Stephen. (2014). The Psychological Flexibility Model: A Basis for Integration and Progress in Psychological Approaches to Chronic Pain Management. The Journal of Pain, 15(3), 221-234. doi: http://dx.doi.org/10.1016/j.jpain.2013.10.014

McCracken, Lance M., \& Samuel, Victoria M. (2007a). The role of avoidance, pacing, and other activity patterns in chronic pain. Pain Vol 130(1-2) Jul 2007, 119-125.

McCracken, Lance M., Spertus, Ilyse L., Janeck, Amy S., Sinclair, Donald, \& Wetzel, F. (1999). Behavioral dimensions of adjustment in persons with chronic pain: Painrelated anxiety and acceptance. Pain, 80(1-2), 283-289. doi: dx.doi.org/10.1016/S0304-3959(98)00219-X

McCracken, Lance M., \& Velleman, Sophie C. (2010). Psychological flexibility in adults with chronic pain: A study of acceptance, mindfulness, and values-based action in primary care. Pain, 148(1), 141-147. doi: http://dx.doi.org/10.1016/j.pain.2009.10.034

McCracken, Lance M., \& Vowles, Kevin E. (2007). Psychological flexibility and traditional pain management strategies in relation to patient functioning with chronic pain: An 
examination of a revised instrument. The Journal of Pain, 8(9), 700-707. doi: http://dx.doi.org/10.1016/j.jpain.2007.04.008

McCracken, Lance M., \& Vowles, Kevin E. (2008). A prospective analysis of acceptance of pain and values-based action in patients with chronic pain. Health Psychology, 27(2), 215-220. doi: http://dx.doi.org/10.1037/0278-6133.27.2.215

McCracken, Lance M., \& Vowles, Kevin E. (2014). Acceptance and Commitment Therapy and Mindfulness for Chronic Pain: Model, Process, and Progress. American Psychologist, 69(2), 178-187. doi: dx.doi.org/10.1037/a0035623

McCracken, Lance M., Vowles, Kevin E., \& Eccleston, Christopher. (2004). Acceptance of chronic pain: Component analysis and a revised assessment method. Pain, 107(1-2), 159-166. doi: http://dx.doi.org/10.1016/j.pain.2003.10.012

McCracken, Lance M., \& Yang, Su-Yin. (2006). The role of values in a contextual cognitivebehavioral approach to chronic pain. Pain, 123(1-2), 137-145. doi: http://dx.doi.org/10.1016/j.pain.2006.02.021

McCracken, Lance, \& Samuel, Victoria. (2007b). The role of avoidance, pacing, and other activity patterns in chronic pain. Pain, 130(1), 119 - 125. doi: dx.doi.org/10.1016/j.pain.2006.11.016

McDermott, Anne M., Toelle, Thomas R., Rowbotham, David J., Schaefer, Caroline P., \& Dukes, Ellen M. (2006). The burden of neuropathic pain: Results from a crosssectional survey. European Journal of Pain, 10(2), 127-135. doi: http://dx.doi.org/10.1016/j.ejpain.2005.01.014

McGrath, Joseph E., \& Johnson, Bettina A. (2003). Methodology makes meaning: How both qualitative and quantitative paradigms shape evidence and its interpretation. In P. M. R. Camic, Jean E; Yardley, Lucy (Ed.), Qualitative research in psychology: Expanding perspectives in methodology and design (pp. 31-48). Washington, DC: American Psychological Association.

McGuire, W.J. (1997). Creative hypothesis generating in psychology: Some useful heuristics. Annual Review of Psychology, 48(1), 1-30. doi: dx.doi.org/10.1146/annurev.psych.48.1.1

McParland, Joanna L., Eccleston, Christopher, Osborn, Mike, \& Hezseltine, Louisa. (2011). It's not fair: An Interpretative Phenomenological Analysis of discourses of justice and fairness in chronic pain. Health: An Interdisciplinary Journal for the Social Study of Health, Illness \& Medicine, 15(5), 459-474. doi: 10.1177/1363459310383593

Mehling, Wolf E., Gopisetty, Viranjini, Bartmess, Elizabeth, Acree, Mike, Pressman, Alice, Goldberg, Harley, ... Avins, Andrew L. (2012). The prognosis of acute low back pain in primary care in the United States: a 2-year prospective cohort study. Spine, 37(8), 678-684. doi: dx.doi.org/10.1097/BRS.0b013e318230ab20

Melzack, R., Coderre, T. J., Katz, J., \& Vaccarino, A. L. (2001). Central neuroplasticity and pathological pain. Annals of the New York Academy of Sciences, 933, 157-174. doi: dx.doi.org/10.1111/j.1749-6632.2001.tb05822.x

Melzack, R., \& Wall, P. D. (1988). The challenge of pain. London: Penguin. 
Melzack, R., \& Wall, P.D. (1967). Pain mechanisms: a new theory. Survey of Anesthesiology, 11(2), 89. doi: dx.doi.org/10.1097/00132586-197212000-00046

Melzack, Ronald, \& Katz, Joel. (2013). Pain. Wiley Interdisciplinary Reviews: Cognitive Science, 4(1), 1-15. doi: 10.1002/wcs.1201

Melzack, Ronald, \& Wall, Patrick D. (1965). Pain mechanisms: a new theory. Science, 150(3699), 971-979. doi: http://dx.doi.org/10.1126/science.150.3699.971

Mercadante, Sebastiano, Villari, Patrizia, Ferrera, Patrizia, \& Arcuri, Edoardo. (2004). Prolonged Uncontrolled Pain, Psychological Distress, and Opioid Escalation. Journal of Pain and Symptom Management 28(1), 1-3. doi: dx.doi.org/10.1016/j.jpainsymman.2004.04.001

Meredith, Pamela, Strong, Jenny, \& Feeney, Judith A. (2006). Adult attachment, anxiety, and pain self-efficacy as predictors of pain intensity and disability. Pain, 123(1), 146-154. doi: http://dx.doi.org/10.1016/j.pain.2006.02.025

Merskey, H, \& Bogduk, N. (1994). Classification of Chronic Pain: descriptions of chronic pain syndromes and definitions of pain terms. Seattle: International Association for the Study of Pain.

Meyer, Jeffrey A. (2010). Integration of the avoidance cycle with the schema enmeshment model of pain: Relationships with quality of life and disability in chronic, nonmalignant pain. Dissertation Abstracts International: Section B: The Sciences and Engineering, 70(10-B), 6559.

Michael, E. S., \& Burns, J. W. (2004). Catastrophizing and pain sensitivity among chronic pain patients: moderating effects of sensory and affect focus. Annals of Behavioral Medicine, 27(3), 185-194. doi: dx.doi.org/10.1207/s15324796abm2703_6

Miles, A., Curran, H. V., Pearce, S., \& Allan, L. (2005). Managing constraint: the experience of people with chronic pain. Social Science \& Medicine, 61(2), 431-441. doi: 10.1016/j.socscimed.2004.11.065

Miller, Steven I., \& Fredericks, Marcel. (1999). How Does Grounded Theory Explain? Qualitative Health Research, 9(4), 538-551. doi: 10.1177/104973299129122054

Mintzberg, H. (2005). Developing theory about the development of theory. Great minds in management: The process of theory development, 355-372.

Miro, Elena, Martinez, Maria Pilar, Sanchez, Ana Isabel, Prados, German, \& Medina, Ana. (2011). When is pain related to emotional distress and daily functioning in fibromyalgia syndrome? The mediating roles of self-efficacy and sleep quality. British Journal of Health Psychology, 16(4), 799-814. doi: dx.doi.org/10.1111/j.20448287.2011.02016.x

Mishel, Merle H. (1999). Uncertainty in Chronic Illness. Annual Review of Nursing Research, 17(1), 269-294.

Mo'tamedi, Hadi, Rezaiemaram, Payman, \& Tavallaie, Abaas. (2012). The effectiveness of a group-based acceptance and commitment additive therapy on rehabilitation of female outpatients with chronic headache: Preliminary findings reducing 3 dimensions of headache impact. Headache: The Journal of Head and Face Pain, 52(7), 1106-1119. 
Molden, Daniel C., \& Dweck, Carol S. (2006). Finding "Meaning" in Psychology: A Lay Theories Approach to Self-Regulation, Social Perception, and Social Development. American Psychologist, 61(3), 192. doi: 10.1037/0003-066X.61.3.192

Moldovan, Adela R., Onac, Ioana A., Vantu, Marian, Szentagotai, Aurora, \& Onac, Ioan. (2009). Emotional distress, pain catastrophizing and expectancies in patients with low back pain. Journal of Cognitive and Behavioral Psychotherapies, 9(1), 83-93.

Molineux, M. L. (2009). The nature of occupation. In M. Curtin, M. Molineux \& J. Supyk (Eds.), Occupational Therapy and Physical Dysfunction: Enabling Occupation (6th ed., pp. 17-26). Edinburgh: Elsevier.

Molton, I. R., Stoelb, B. L., Jensen, M. P., Ehde, D. M., Raichle, K. A., \& Cardenas, D. D. (2009). Psychosocial factors and adjustment to chronic pain in spinal cord injury: replication and cross-validation. Journal of Rehabilitation Research \& Development, 46(1), 31-42.

Monsivais, D. (2005). Self-organization in chronic pain: a concept analysis. Rehabilitation Nursing, 30(4), 147-151. doi: dx.doi.org/10.1002/j.2048-7940.2005.tb00099.x

Montali, Lorenzo, Monica, Colombo, Riva, Paolo, \& Cipriani, Roberto. (2011). Conflicting representations of pain: A qualitative analysis of health care professionals' discourse. Pain Medicine, 12(11), 1585-1593. doi: http://dx.doi.org/10.1111/j.15264637.2011.01252.x

Moos, Rudolf H., \& Holahan, Charles J. (2003). Dispositional and Contextual Perspectives on Coping: Toward an Integrative Framework. Journal of Clinical Psychology, 59(12), 1387-1403. doi: dx.doi.org/10.1002/jclp.10229

Moos, Rudolf H., \& Holahan, Charles J. (2007). Adaptive tasks and methods of coping with illness and disability. In E. Martz (Ed.), Coping with Chronic Illness and Disability: Theoretical, Empirical, and Clinical Aspects (pp. 107-126). New York. NY: Springer Science+Business Media, LLC.

Morden, Andrew, Jinks, Clare, \& Ong, Bie Nio. (2011). Lay models of self-management: How do people manage knee osteoarthritis in context? Chronic Illness, 7(3), 185-200.

Morgan, Christopher Ll, Conway, Pete, \& Currie, Craig J. (2011). The relationship between self-reported severe pain and measures of socio-economic disadvantage. European Journal of Pain, 15(10), 1107-1111. doi: dx.doi.org/10.1016/j.ejpain.2011.04.010

Morinis, Alan. (1985). The Ritual Experience: Pain and the Transformation of Consciousness in Ordeals of Initiation. Ethos, 13(2), 150-174. doi: 10.1525/eth.1985.13.2.02a00040

Morley, S., Shapiro, D. A., \& Biggs, J. (2004). Developing a treatment manual for attention management in chronic pain. Cognitive Behaviour Therapy, 33(1), 1-11. doi: 10.1080/16506070310001794

Morley, S., Williams, A., \& Hussain, S. (2008). Estimating the clinical effectiveness of cognitive behavioural therapy in the clinic: evaluation of a CBT informed pain management programme. Pain, 137(3), 670-680. doi: dx.doi.org/10.1016/j.pain.2008.02.025 
Morley, Stephen, Davies, Caitlin, \& Barton, Stephen. (2005). Possible selves in chronic pain: Self-pain enmeshment, adjustment and acceptance. Pain, 115(1-2), 84-94. doi: http://dx.doi.org/10.1016/j.pain.2005.02.021

Mortimer, Monica, Ahlberg, Gunnel, \& Group, M. USIC-Norrtalje Study. (2003). To seek or not to seek? Care-seeking behaviour among people with low-back pain. Scandinavian Journal of Public Health, 31(3), 194-203. doi: dx.doi.org/10.1080/14034940210134086

Moscucci, O. (2003). Holistic obstetrics: the origins of "natural childbirth" in Britain. Postgraduate Medical Journal, 79(929), 168-173. doi: 10.1136/pmj.79.929.168

Moskovitz, Peter A. (2011). Understanding suffering: The phenomenology and neurobiology of the experience of illness and pain. In J. E. Giordano (Ed.), Maldynia:

Multidisciplinary Perspectives on the Illness of Chronic Pain (pp. 33-64). Bosa Roca, USA: Taylor \& Francis Inc.

Moss-Morris, Rona, Petrie, Keith J., \& Weinman, John. (1996). Functioning in chronic fatigue syndrome: Do illness perceptions play a regulatory role? British Journal of Health Psychology, 1(1), 15-25. doi: 10.1111/j.2044-8287.1996.tb00488.x

Mudge, Suzie, Kayes, Nicola M., Stavric, Verna A., Channon, Alexis S., Kersten, Paula, \& McPherson, Kathryn M. (2013). Living well with disability: Needs, values and competing factors. The International Journal of Behavioral Nutrition and Physical Activity Vol 10 Aug 2013, ArtID 100, 10.

Mullady, Daniel K, Yadav, Dhiraj, Amann, Stephen T, O'Connell, Michael R, Barmada, Michael M, Elta, Grace H, . . Korneffel, Meredith L. (2011). Type of pain, painassociated complications, quality of life, disability and resource utilisation in chronic pancreatitis: a prospective cohort study. Gut, 60(1), 77-84. doi: dx.doi.org/10.1136/gut.2010.213835

Murinson, B. B., Agarwal, A. K., \& Haythornthwaite, J. A. (2008). Cognitive expertise, emotional development, and reflective capacity: clinical skills for improved pain care. Journal of Pain, 9(11), 975-983. doi: dx.doi.org/10.1016/j.jpain.2008.07.010

Nakamura, Masaya, Nishiwaki, Yuji, Ushida, Takahiro, \& Toyama, Yoshiaki. (2011). Prevalence and characteristics of chronic musculoskeletal pain in Japan. Journal of Orthopaedic Science, 16(4), 424-432. doi: 10.1007/s00776-011-0102-y

Nathaniel, A. (2011). An integrated philosophical framework that fits grounded theory. In V. B. Martin \& A. Gynnild (Eds.), Grounded Theory: The philosophy, method and work of Barney Glaser (pp. 187-200). Boca Raton, Florida: Brown Walker Press.

Nes, Lise Solberg, Roach, Abbey R., \& Segerstrom, Suzanne C. (2009). Executive functions, self-regulation, and chronic pain: A review. Annals of Behavioral Medicine, 37(2), 173-183. doi: http://dx.doi.org/10.1007/s12160-009-9096-5

Ness, Sheryl M. (2009). Pain expression in the perioperative period: insights from a focus group of Somali women. Pain Management Nursing, 10(2), 65-75. doi: http://dx.doi.org/10.1016/j.pmn.2008.05.001 
Neugebauer, V., Galhardo, V., Maione, S., \& Mackey, S. C. (2009). Forebrain pain mechanisms. Brain Research Reviews, 60(1), 226-242. doi:

dx.doi.org/10.1016/j.brainresrev.2008.12.014

Nguyen, Marisa, Ugarte, Carlos, Fuller, Ivonne, Haas, Gregory, \& Portenoy, Russell K. (2005). Access to Care for Chronic Pain: Racial and Ethnic Differences. The Journal of Pain, 6(5), 301-314. doi: http://dx.doi.org/10.1016/j.jpain.2004.12.008

Nicholas, M. K., Coulston, C. M., Asghari, A., \& Malhi, G. S. (2009). Depressive symptoms in patients with chronic pain. Medical Journal of Australia, 190(7 Suppl), S66-70.

Nicholas, Michael, \& Asghari, Ali. (2007). "Investigating acceptance in adjustment to chronic pain: Is acceptance broader than we thought?": Response to letter by McCracken et al. (2006). Pain, 128(3), 284-285. doi: http://dx.doi.org/10.1016/j.pain.2006.10.003

Nicholas, Michael K., Linton, Steven J., Watson, Paul J., \& Main, Chris J. (2011). Early identification and management of psychological risk factors ("yellow flags") in patients with low back pain: a reappraisal. Physical Therapy, 91(5), 737-753.

Nicholson Perry, K., Nicholas, M. K., Middleton, J., \& Siddall, P. (2009). Psychological characteristics of people with spinal cord injury-related persisting pain referred to a tertiary pain management center. Journal of Rehabilitation Research \& Development, 46(1), 57-67.

Nielson, Warren R. PhD, Jensen, Mark P. PhD, Karsdorp, Petra A. PhD, \& Vlaeyen, Johannes W. S. PhD. (2013). Activity Pacing in Chronic Pain: Concepts, Evidence, and Future Directions. Clinical Journal of Pain, 29(5), 461-468. doi: dx.doi.org/10.1097/AJP.0b013e3182608561

Noel, Melanie, Petter, Mark, \& Chambers, Christine T. PhD. (2012). Cognitive Behavioral Therapy for Pediatric Chronic Pain: The Problem, Research, and Practice. Journal of Cognitive Psychotherapy, 26(2), 143-156. doi: 10.1016/j.pain.2005.10.027.

Oades, Lindsay, Deane, Frank, Crowe, Trevor, Lambert, W., Kavanagh, David, \& Lloyd, Chris. (2005). Collaborative recovery: An integrative model for working with individuals who experience chronic and recurring mental illness. Australasian Psychiatry, 13(3), 279-284. doi: dx.doi.org/10.1111/j.1440-1665.2005.02202.x

Occam's razor - Wikipedia, the free encyclopedia. (2014).

Ohayon, Maurice M., \& Stingl, Julia C. (2012). Prevalence and comorbidity of chronic pain in the German general population. Journal of Psychiatric Research, 46(4), 444-450. doi: dx.doi.org/10.1016/j.jpsychires.2012.01.001

Oliveira, Daniela R., Leite, Alice A., \& Rocha-Filho, Pedro A. (2011). Which patients with headache do not seek medical attention? Headache: The Journal of Head and Face Pain, 51(8), 1279-1284. doi: http://dx.doi.org/10.1111/j.1526-4610.2011.01977.x

Oliver, Carolyn. (2012). Critical Realist Grounded Theory: A New Approach for Social Work Research. British Journal of Social Work, 42(2), 371-387. doi: 10.1093/bjsw/bcr064 
Oliver, M. (1996). Understanding disability: From theory to practice. New York, NY: St Martin's Press.

Ong, Beng Kok. (2011). Grounded Theory Method (GTM) and the Abductive Research Strategy (ARS): a critical analysis of their differences. International Journal of Social Research Methodology, 15(5), 417-432. doi: 10.1080/13645579.2011.607003

Osborne, T. L., Jensen, M. P., Ehde, D. M., Hanley, M. A., \& Kraft, G. (2007). Psychosocial factors associated with pain intensity, pain-related interference, and psychological functioning in persons with multiple sclerosis and pain. Pain, 127(1-2), 52-62. doi: dx.doi.org/10.1016/j.pain.2006.07.017

Ownsworth, T., Hawkes, A., Steginga, S., Walker, D., \& Shum, D. (2009). A biopsychosocial perspective on adjustment and quality of life following brain tumor: a systematic evaluation of the literature. Disability \& Rehabilitation, 31(13), 1038-1055. doi: $10.1080 / 09638280802509538$

Paananen, Markus V., Taimela, Simo P., Tammelin, Tuija H., Kantomaa, Marko T., Ebeling, Hanna E., Taanila, Anja M., . . . Karppinen, Jaro I. (2011). Factors related to seeking health care among adolescents with musculoskeletal pain. Pain, 152(4), 896-903. doi: http://dx.doi.org/10.1016/j.pain.2011.01.002

Paez-Blarrina, Marisa, Luciano, Carmen, Gutierrez-Martinez, Olga, Valdivia, Sonsoles, Rodriguez-Valverde, Miguel, \& Ortega, Jose. (2008). Coping with pain in the motivational context of values: Comparison between an acceptance-based and a cognitive control--based protocol. Behavior Modification 32(3), 403-422. doi: $10.1177 / 0145445507309029$

Paley, J. (2011). The fictionalist paradigm. Nursing Philosophy, 12(1), 53-66.

Parry, R. (2004). Communication during goal-setting in physiotherapy treatment sessions. Clinical Rehabilitation, 18(6), 668-682. doi: dx.doi.org/10.1191/0269215504cr745oa

Party, IASP Taxonomy Working. (2014, May 22, 2012). IASP Taxonomy - IASP. Retrieved 25 November, 2014, from http://www.iasppain.org/Education/Content.aspx?ItemNumber=1698\&navItemNumber=576

Patterson, David R. (2005). Behavioral Methods for Chronic Pain and Illness: A Reconsideration and Appreciation. Rehabilitation Psychology, 50(3), 312-315. doi: 10.1037/0090-5550.50.3.312

Patton, M.Q. (1980). Qualitative evaluation methods. Beverly Hills, CA: Sage publications

Pearce, D. (2008, 2008). Utopian Surgery: Early arguments against anaesthesia in surgery, dentistry and childbirth. Retrieved 03 October 2012, 2012, from http://www.generalanaesthesia.com/

Peirce, C. S. (1867). On a New List of Categories. Proceedings of the American Academy of Arts and Sciences, 7, 287-298. doi: 10.2307/20179567

Pellegrino, E.D. (2011). A clinical ethics of chronic pain management: Basis, reason, and responsibilities. In J. Giordano (Ed.), Maldynia: Multidisciplinary perspectives on the illness of chronic pain (pp. 213-219). Boca Raton, Florida: CRC Press, Taylor \& Francis Group. 
Pentland, W., Walker, J., Minnes, P., Tremblay, M., Brouwer, B., \& Gould, M. (2003). Occupational responses to mid-life and aging in women with disabilities. Journal of Occupational Science, 10(1), 21-30. doi: dx.doi.org/10.1080/14427591.2003.9686507

Perreault, Kadija, \& Dionne, Clermont E. (2005). Patient-physiotherapist agreement in low back pain. Journal of Pain, 6(12), 817-828. doi: dx.doi.org/10.1016/j.jpain.2005.07.008

Perret, Danielle, \& Rosen, Charles. (2011). A Physician-Driven Solution-The Association for Medical Ethics, The Physician Payment Sunshine Act, and Ethical Challenges in Pain Medicine. Pain Medicine, 12(9), 1361-1375. doi: 10.1111/j.15264637.2011.01217.x

Persson, D., Eklund, M., \& Isacsson, A. (1999). The experience of everyday occupations and its relation to sense of coherence -- a methodological study. Journal of Occupational Science, 6(1), 13-26. doi: dx.doi.org/10.1080/14427591.1999.9686447

Peters, Sarah, Rogers, Anne, Salmon, Peter, Gask, Linda, Dowrick, Chris, Towey, Maria, .. . Morriss, Richard. (2009). What do patients choose to tell their doctors? Qualitative analysis of potential barriers to reattributing medically unexplained symptoms. Journal of General Internal Medicine, 24(4), 443-449. doi: dx.doi.org/10.1007/s11606-008-0872-x

Pfizer. (2012). Health Report: Chronic Pain (pp. 20). New Zealand: Pfizer New Zealand Ltd.

Phelan, S., \& Kinsella, E. A. (2009). Occupational identity: engaging socio-cultural perspectives. Journal of Occupational Science, 16(2), 85-91. doi: dx.doi.org/10.1080/14427591.2009.9686647

Philips, C, \& Hunter, M. (1981). Pain behavior in headache sufferers. Behavioural Analysis and Modification, 4(4), 257-266.

Philips, H. (1987). Avoidance behaviour and its role in sustaining chronic pain. Behaviour Research and Therapy, 25(4), 273-279. doi: http://dx.doi.org/10.1016/00057967(87)90005-2

Phillips, Catherine. (2007). Pain(ful) subjects: Regulated bodies in medicine and social work. Qualitative Social Work: Research and Practice, 6(2), 197-212. doi: dx.doi.org/10.1177/1473325007077253

Pierce, D. (2001). Untangling occupation and activity. American Journal of Occupational Therapy, 55(2), 138-146. doi: dx.doi.org/10.5014/ajot.55.2.138

Pincus, Tamar, Smeets, Rob J., Simmonds, Maureen J. , \& Sullivan, Michael J. L. (2010). The Fear Avoidance Model Disentangled: Improving the Clinical Utility of the Fear Avoidance Model. Clinical Journal of Pain November/December, 26(9), 739-746. doi: dx.doi.org/10.1097/AJP.0b013e3181f15d45

Pincus, Tamar, \& Morley, Stephen. (2001). Cognitive-Processing Bias in Chronic Pain: A Review and Integration. Psychological Bulletin, 127(5), 599-617. doi: dx.doi.org/10.1037//0033-2909.127.5.599 
Ploner, Markus, Lee, Michael C., Wiech, Katja, Bingel, Ulrike, \& Tracey, Irene. (2011). Flexible Cerebral Connectivity Patterns Subserve Contextual Modulations of Pain. Cerebral Cortex, 21(3), 719-726. doi: 10.1093/cercor/bhq146

Polkinghorne, D.E. (2005). Language and meaning: Data collection in qualitative research. Journal of counseling psychology, 52(2), 137. doi: dx.doi.org/10.1037/00220167.52.2.137

Prkachin, K. M., Schultz, I. Z., \& Hughes, E. (2007). Pain behavior and the development of pain-related disability: the importance of guarding. Clinical Journal of Pain, 23(3), 270-277. doi: dx.doi.org/10.1097/AJP.0b013e3180308d28

Pullman, Daryl. (2002). Human dignity and the ethics and aesthetics of pain and suffering. Theoretical Medicine \& Bioethics, 23(1), 75-94.

Quartana, Phillip J., Campbell, Claudia M., \& Edwards, Robert R. (2009). Pain catastrophizing: a critical review. Expert Review of Neurotherapeutics, 9(5), 745-758. doi: 10.1586/ern.09.34

Rabin, D. L., \& Schach, E. (1975). Medicaid, morbidity, and physician use. Medical Care, 13(1), 68-78. doi: dx.doi.org/10.1097/00005650-197501000-00007

Raftery, Miriam N., Ryan, Padhraig, Normand, Charles, Murphy, Andrew W., de la Harpe, Davida, \& McGuire, Brian E. (2012). The economic cost of chronic noncancer pain in Ireland: results from the PRIME study, part 2. Journal of Pain, 13(2), 139-145. doi: dx.doi.org/10.1016/j.jpain.2011.10.004

Raheim, Malfrid, \& Haland, Wenche. (2006). Lived Experience of Chronic Pain and Fibromyalgia: Women's Stories From Daily Life. Qualitative Health Research, 16(6), 741-761. doi: dx.doi.org/10.1177/1049732306288521

Ramirez-Maestre, C., Esteve, R., \& Lopez, A. E. (2012). The role of optimism and pessimism in chronic pain patients adjustment. Spanish Journal of Psychology, 15(1), 286-294. doi: dx.doi.org/10.5209/rev_SJOP.2012.v15.n1.37335

Ramirez-Maestre, Carmen, Esteve, Rosa, \& Lopez, Alicia E. (2008). Cognitive appraisal and coping in chronic pain patients. European Journal of Pain, 12(6), 749-756. doi: http://dx.doi.org/10.1016/j.ejpain.2007.11.004

Ramon, Shulamit, Healy, Bill, \& Renouf, Noel. (2007). Recovery from Mental Illness as an Emergent Concept and Practice in Australia and the UK. International Journal of Social Psychiatry, 53(2), 108-122. doi: dx.doi.org/10.1177/0020764006075018

Rebeiro, K. L., \& Polgar, J. M. (1999). Enabling occupational performance: optimal experiences in therapy. Canadian Journal of Occupational Therapy, 66(1), 14-22. doi: dx.doi.org/10.1177/000841749906600102

Reed, K., Hocking, C., \& Smythe, L. (2010). The Interconnected Meanings of Occupation: The Call, Being-With, Possibilities. Journal of Occupational Science, 17(3), 140-149. doi: dx.doi.org/10.1080/14427591.2010.9686688

Reid, Denise. (2011). Mindfulness and flow in occupational engagement: Presence in doing. The Canadian Journal of Occupational Therapy, 78(1), 50-56. doi: 10.1093/clipsy/bpg015 
Reiner, Keren, Tibi, Lee, \& Lipsitz, Joshua D. (2013). Do Mindfulness-Based Interventions Reduce Pain Intensity? A Critical Review of the Literature. Pain Medicine, 14(2), 230-242. doi: 10.1111/pme.12006

Reinseth, Lillian, Kjeken, Ingvild, Uhlig, Till, \& Espnes, Geir. (2012). Participation in committed and discretionary activities and quality of life in women with rheumatoid arthritis. British Journal of Occupational Therapy, 75(7), 313-320. doi: http://dx.doi.org/10.4276/030802212X13418284515794

Reis, Shmuel, Hermoni, Doron, Van-Raalte, Riki, Dahan, Rachel, \& Borkan, Jeffrey M. (2007). Aggregation of qualitative studies--From theory to practice: Patient priorities and family medicine/general practice evaluations. Patient Education and Counseling, 65(2), 214-222. doi: dx.doi.org/10.1016/j.pec.2006.07.011

Reisner, Andrew D. (2005). The Common Factors, Empirically Validated Treatments, and Recovery Models of Therapeutic Change. Psychological Record, 55(3), 377-399.

Reitsma, Michelle, Tranmer, Joan E., Buchanan, Diane M., \& VanDenKerkhof, Elizabeth G. (2012). The epidemiology of chronic pain in Canadian men and women between 1994 and 2007: longitudinal results of the National Population Health Survey. Pain Research \& Management, 17(3), 166-172.

Resnick, Sandra G., \& Rosenheck, Robert A. (2006). Recovery and positive psychology: parallel themes and potential synergies. Psychiatric Services, 57(1), 120-122. doi: dx.doi.org/10.1176/appi.ps.57.1.120

Reynolds, F. (2003). Reclaiming a positive identity in chronic illness through artistic occupation. OTJR: Occup Particip Health, 23, 118-127.

Richardson, Jane C., Ong, Bie Nio, \& Sim, Julius. (2006). Is chronic widespread pain biographically disruptive? Social Science \& Medicine, 63(6), 1573-1585. doi: http://dx.doi.org/10.1016/j.socscimed.2006.03.040

Richardson, Rudy, \& Kramer, Eric Hans. (2006). Abduction as the type of inference that characterizes the development of a grounded theory. Qualitative Research, 6(4), 497513. doi: http://dx.doi.org/10.1177/1468794106068019

Riess, Helen. (2010). Empathy in medicine--a neurobiological perspective. JAMA, 304(14), 1604-1605. doi: dx.doi.org/10.1001/jama.2010.1455

Risdon, Andrea, Eccleston, Chris, Crombez, Geert, \& McCracken, Lance. (2003). How can we learn to live with pain?: A Q-methodological analysis of the diverse understandings of acceptance of chronic pain. Social Science \& Medicine, 56(2), 375386. doi: dx.doi.org/10.1016/S0277-9536(02)00043-6

Roelofs, J., Peters, M. L., \& Vlaeyen, J. W. (2002). Selective attention for pain-related information in healthy individuals: the role of pain and fear. European Journal of Pain: Ejp, 6(5), 331-339. doi: dx.doi.org/10.1016/S1090-3801(02)00021-6

Rollin, Bernard E. (1999). Some conceptual and ethical concerns about current views of pain. Pain Forum, 8(2), 78-83. doi: http://dx.doi.org/10.1016/S1082-3174(99)70050-4 
Rollman, Gary B. (2004). Ethnocultural Variations in the Experience of Pain. Pain: Psychological perspectives (pp. 155-178). Mahwah, NJ: Lawrence Erlbaum Associates Publishers; US.

Romano, Joan M., Syrjala, Karen L., Levy, Ronal L., Turner, Judith A., Evans, Patricia, \& Keefe, Francis J. (1988). Overt pain behaviors: Relationship to patient functioning and treatment outcome. Behavior Therapy, 19(2), 191-201. doi: http://dx.doi.org/10.1016/S0005-7894(88)80042-X

Rosenbaum, M.S. (2011). From theoretical generation to verification using structural equation modeling. In V. B. Martin \& A. Gynnild (Eds.), Grounded theory: The philosophy, method and work of Barney Glaser (pp. 283-296). Boca Raton, Florida: Brown Walker Press.

Roth, M. L., Tripp, D. A., Harrison, M. H., Sullivan, M., \& Carson, P. (2007). Demographic and psychosocial predictors of acute perioperative pain for total knee arthroplasty. Pain Research \& Management, 12(3), 185-194.

Roth, R. S., Geisser, M E, \& Williams, D. A. (2012). Interventional pain medicine: retreat from the biopsychosocial model of pain. Translational Behavioral Medicine, 2(1), 106-116. doi: 10.1007/s13142-011-0090-7

Rowles, G D. (2008). Place in occupational science: a life course perspective on the role of environmental context in the quest for meaning. Journal of Occupational Science: Australia, 15(3), 127-135. doi: dx.doi.org/10.1080/14427591.2008.9686622

Ruehlman, Linda S., Karoly, P., Newton, C., \& Aitken, Leona S. (2005). The development and preliminary validation of a brief measure of chronic pain impact for use in the general population. Pain, 113(1-2), 82-89. doi: dx.doi.org/10.1016/j.pain.2004.09.037

Ruscio, John. (2010). Irrational beliefs stemming from judgment errors: Cognitive limitations, biases, and experiential learning. [References]. In D. David, S. J. Lynn \& A. Ellis (Eds.), Rational and irrational beliefs: Research, theory, and clinical practice (pp. 291-312). New York, NY: Oxford University Press; US.

Rusk, Reuben D., \& Waters, Lea E. (2013). Tracing the size, reach, impact, and breadth of positive psychology. The Journal of Positive Psychology, 8(3), 207-221. doi: 10.1080/17439760.2013.777766

Ryan, Richard M., Huta, Veronika, \& Deci, Edward L. (2013). Living well: A selfdetermination theory perspective on eudaimonia. Delle Fave, Antonella [Ed] (2013) The exploration of happiness: Present and future perspectives (pp 117-139) ix, 340 pp New York, NY, US: Springer Science + Business Media; US, 117-139.

Saldaña, J. (2013). The coding manual for qualitative researchers. London: Sage Publications Limited.

Salick, Elizabeth C., \& Auerbach, Carl F. (2006). From Devastation to Integration: Adjusting to and Growing From Medical Trauma. Qualitative Health Research, 16(8), 10211037. doi: http://dx.doi.org/10.1177/1049732306292166

Salpakoski, Anu, Portegijs, Erja, Kallinen, Mauri, Sihvonen, Sanna, Kiviranta, Ilkka, Alen, Markku, ... Sipila, Sarianna. (2010). Physical inactivity and pain in older men and 
women with hip fracture history. Gerontology, 57(1), 19-27. doi:

dx.doi.org/10.1159/000315490

Salvadorini, G., Bandinelli, F., Delle Sedie, A., Riente, L., Candelieri, A., Generini, S., . . . Matucci-Cerinic, M. (2012). Ankylosing spondylitis: how diagnostic and therapeutic delay have changed over the last six decades. Clinical \& Experimental Rheumatology, $30(4), 561-565$.

Samwel, Han JA, Kraaimaat, Floris W, Evers, Andrea WM, \& Crul, Ben JP. (2007). The role of fear-avoidance and helplessness in explaining functional disability in chronic pain: a prospective study. International journal of behavioral medicine, 14(4), 237-241. doi: dx.doi.org/10.1007/BF03002998

Sandgren, Anna, Thulesius, Hans, Petersson, Kerstin, \& Fridlund, Bengt. (2010). Living on hold in palliative cancer care. The Grounded Theory Review, 9(1), 79-100.

Sauer, Shannon E., Burris, Jessica L., \& Carlson, Charles R. (2010). New directions in the management of chronic pain: Self-regulation theory as a model for integrative clinical psychology practice. Clinical Psychology Review, 30(6), 805-814. doi: http://dx.doi.org/10.1016/i.cpr.2010.06.008

Schmitt, M. A., van Meeteren, N. L., de Wijer, A., van Genderen, F. R., van der Graaf, Y., \& Helders, P. J. (2009). Patients with chronic whiplash-associated disorders: relationship between clinical and psychological factors and functional health status. American Journal of Physical Medicine \& Rehabilitation, 88(3), 231-238. doi: dx.doi.org/10.1097/PHM.0b013e318198b684

Schrooten, Martien G. S., Van Damme, Stefaan, Crombez, Geert, Peters, Madelon L., Vogt, Julia, \& Vlaeyen, Johan W. S. (2012). Nonpain goal pursuit inhibits attentional bias to pain. Pain, 153(6), 1180-1186. doi: http://dx.doi.org/10.1016/j.pain.2012.01.025

Schwab, Abraham P. (2008). Putting cognitive psychology to work: Improving decisionmaking in the medical encounter. Social Science \& Medicine, 67(11), 1861-1869. doi: dx.doi.org/10.1016/j.socscimed.2008.09.005

Schwarz, Norbert. (2007). Retrospective and concurrent self-reports: The rationale for realtime data capture. In A. A. Stone, S. Shiffman, A. A. Atienza \& L. Nebeling (Eds.), The science of real-time data capture: Self-reports in health research (pp. 11-26). New York: Oxford.

Schwenk, Charles R. (1984). Cognitive simplification processes in strategic decision-making. Strategic Management Journal, 5(2), 111-128. doi: 10.1002/smj.4250050203

Scott, Helen MP. (2007). The temporal integration of connected study into a structured life: a grounded theory. University of Portsmouth.

Seery, Mark D. (2011). Resilience: A Silver Lining to Experiencing Adverse Life Events? Current Directions in Psychological Science, 20(6), 390-394. doi: $10.1177 / 0963721411424740$

Seligman, M.E.P., \& Csikszentmihalyi, M. (2000). Positive psychology: an introduction. American Psychologist, 55(1), 5. doi: dx.doi.org/10.1037//0003-066X.55.1.5 
Seligman, Martin E., \& Csikszentmihalyi, Mihaly. (2001). "Positive psychology: An introduction": Reply. American Psychologist 56(1), 89-90.

Seligman, Martin E., Rashid, Tayyab, \& Parks, Acacia C. (2006). Positive Psychotherapy. American Psychologist 61(8), 774-788. doi: dx.doi.org/10.1037/0003-066X.61.8.774

Severeijns, R., Vlaeyen, J. W., van den Hout, M. A., \& Weber, W. E. (2001). Pain catastrophizing predicts pain intensity, disability, and psychological distress independent of the level of physical impairment. Clinical Journal of Pain., 17(2), 165-172. doi: dx.doi.org/10.1097/00002508-200106000-00009

Shah, S.K., \& Corley, K.G. (2006a). Building Better Theory by Bridging the QuantitativeQualitative Divide*. Journal of Management Studies, 43(8), 1821-1835.

Shah, Sonali K., \& Corley, Kevin G. (2006b). Building Better Theory by Bridging the Quantitative-Qualitative Divide. Journal of Management Studies, 43(8), 1821-1835. doi: http://dx.doi.org/10.1111/j.1467-6486.2006.00662.x

Shank, K, \& Cutchin, M. (2010). Transactional occupations of older women aging-in-place: Negotiating change and meaning. Journal of Occupational Science: Australia, 17, 413. doi: dx.doi.org/10.1080/14427591.2010.9686666

Shapiro, Barbara S. (1999). Implications for our definitions of pain. Pain Forum, 8(2), 100102. doi: http://dx.doi.org/10.1016/S1082-3174(99)70034-6

Siddall, Philip J., \& Cousins, Michael J. (2004). Persistent Pain as a Disease Entity: Implications for Clinical Management. Anesthesia \& Analgesia, 99(2), 510-520. doi: 10.1213/01.ane.0000133383.17666.3a

Silver, Christina, \& Patashnick, Jennifer. (2011). Finding Fidelity: Advancing Audiovisual Analysis Using Software. Forum Qualitative Sozialforschung / Forum: Qualitative Social Research, 12(1). http://www.qualitativeresearch.net/index.php/fqs/article/view/1629

Sim, Julius, \& Madden, Sue. (2008). Illness experience in fibromyalgia syndrome: a metasynthesis of qualitative studies. Social Science \& Medicine, 67(1), 57-67. doi: dx.doi.org/10.1016/j.socscimed.2008.03.003

Skeat, J., \& Perry, A. (2008). Grounded theory as a method for research in speech and language therapy. International Journal of Language \& Communication Disorders, 43(2), 95-109. doi: dx.doi.org/10.1080/13682820701437245

Skillgate, Eva, Vingard, Eva, Josephson, Malin, Theorell, Tores, \& Alfredsson, Lars. (2007). The role of coping style in the onset of a new episode of low back and neck/shoulder pain. Psychotherapy and Psychosomatics, 76(4), 253-255.

Skomo, Monica L., Desselle, Shane P., \& Berdine, Hildegarde J. (2006). Factors Influencing Migraineur-Consulting Behavior in a University Population. Headache: The Journal of Head and Face Pain, 46(5), 742-749. doi: http://dx.doi.org/10.1111/j.1526$\underline{4610.2006 .00431 . x}$

Skuladottir, Hafdis, \& Halldorsdottir, Sigridur. (2011). The quest for well-being: Selfidentified needs of women in chronic pain. Scandinavian Journal of Caring Sciences, 25(1), 81-91. doi: http://dx.doi.org/10.1111/j.1471-6712.2010.00793.x 
Slade, Susan Carolyn, Molloy, Elizabeth, \& Keating, Jennifer Lyn. (2009). Stigma experienced by people with nonspecific chronic low back pain: A qualitative study. Pain Medicine, 10(1), 143-154. doi: http://dx.doi.org/10.1111/j.15264637.2008.00540.x

Smajdor, Anna, Stockl, Andrea, \& Salter, Charlotte. (2011). The limits of empathy: problems in medical education and practice. Journal of Medical Ethics, 37(6), 380-383. doi: 10.1136/jme.2010.039628

Smith, Blair H., \& Torrance, Nicola. (2012). Epidemiology of neuropathic pain and its impact on quality of life. Current Pain \& Headache Reports, 16(3), 191-198. doi: dx.doi.org/10.1007/s11916-012-0256-0

Smith, Bruce W., Dalen, Jeanne, Wiggins, Kathryn, Tooley, Erin, Christopher, Paulette, \& Bernard, Jennifer. (2008). The Brief Resilience Scale: Assessing the ability to bounce back. International Journal of Behavioral Medicine, 15(3), 194-200. doi: http://dx.doi.org/10.1080/10705500802222972

Smith, Bruce W., Tooley, Erin M., Montague, Erica Q., Robinson, Amanda E., Cosper, Cynthia J., \& Mullins, Paul G. (2009). The role of resilience and purpose in life in habituation to heat and cold pain. The Journal of Pain, 10(5), 493-500. doi: http://dx.doi.org/10.1016/j.jpain.2008.11.007

Smith, Bruce W., \& Zautra, Alex J. (2004). The Role of Purpose in Life in Recovery from Knee Surgery. International Journal of Behavioral Medicine, 11(4), 197-202. doi: 10.1207/s15327558ijbm1104_2

Smith, Bruce W., \& Zautra, Alex J. (2008). Vulnerability and resilience in women with arthritis: Test of a two-factor model. Journal of Consulting and Clinical Psychology, 76(5), 799-810. doi: dx.doi.org/10.1037/0022-006X.76.5.799

Smith, D. M., Brown, S. L., \& Ubel, P. A. (2008). Mispredictions and misrecollections: challenges for subjective outcome measurement. Disability \& Rehabilitation, 30(6), 418-424. doi: dx.doi.org/10.1080/09638280701625237

Smith, Russell C., \& Bartholomew, Thomas. (2006). Will Hospitals Recover?: The Implications of a Recovery-Orientation. American Journal of Psychiatric Rehabilitation, 9(2), 85-100. doi: dx.doi.org/10.1080/15487760600875982

Smith, T, Ludwig, F, Andersen, L, \& Copolillo, A. (2009). Engagement in occupation and adaptation to low vision. Occupational Therapy in Health Care, 23(2), 119-133. doi: dx.doi.org/10.1080/07380570902788782

Snelgrove, Sherrill, \& Liossi, Christina. (2009). An interpretative phenomenological analysis of living with chronic low back pain. British Journal of Health Psychology, 14(4), 735-749. doi: 10.1348/135910709X402612

Snow-Turek, A. Lynn, Norris, Margaret P., \& Tan, Gabriel. (1996). Active and passive coping strategies in chronic pain patients. Pain, 64(3), 455-462. doi: 10.1016/03043959(95)00190-5

Sofaer, B., Moore, A. P., Holloway, I., Lamberty, J. M., Thorp, T. A. S., \& O'Dwyer, J. (2005). Chronic pain as perceived by older people: a qualitative study. Age and Ageing 34(5), 462-466. doi: dx.doi.org/10.1093/ageing/afi139 
Sorbi, M. J., Peters, M. L., Kruise, D. A., Maas, C. J., Kerssens, J. J., Verhaak, P. F., \& Bensing, J. M. (2006a). Electronic momentary assessment in chronic pain I: psychological pain responses as predictors of pain intensity. Clinical Journal of Pain, 22(1), 55-66. doi: dx.doi.org/10.1097/01.ajp.0000148624.46756.fa

Sorbi, M. J., Peters, M. L., Kruise, D. A., Maas, C. J., Kerssens, J. J., Verhaak, P. F., \& Bensing, J. M. (2006b). Electronic momentary assessment in chronic pain II: pain and psychological pain responses as predictors of pain disability. Clinical Journal of Pain, 22(1), 67-81. doi: dx.doi.org/10.1097/01.ajp.0000148625.84874.48

Stanley, M., \& Cheek, J. (2003). Grounded theory: Exploiting the potential for occupational therapy. The British Journal of Occupational Therapy, 66(4), 143-150.

Stanos, Steven. (2012). Focused review of interdisciplinary pain rehabilitation programs for chronic pain management. Current Pain \& Headache Reports, 16(2), 147-152. doi: http://dx.doi.org/10.1007/s11916-012-0252-4

Steblay, N.M. (1997). Social influence in eyewitness recall: A meta-analytic review of lineup instruction effects. Law and Human Behavior; Law and Human Behavior, 21(3), 283. doi: dx.doi.org/10.1023/A:1024890732059

Stewart, Donna E., \& Yuen, Tracy. (2011). A systematic review of resilience in the physically ill. Psychosomatics, 52(3), 199-209. doi: dx.doi.org/10.1016/j.psym.2011.01.036

Stojanovic, Jane. (2008). Midwifery in New Zealand 1904-1971. Contemporary Nurse: A Journal for the Australian Nursing Profession, 30(2), 156-167. doi: dx.doi.org/10.5172/conu.673.30.2.156

Stone, Arthur A., Broderick, Joan E., Shiffman, Saul S., \& Schwartz, Joseph E. (2004). Understanding recall of weekly pain from a momentary assessment perspective: absolute agreement, between- and within-person consistency, and judged change in weekly pain. Pain, 107(1-2), 61-69. doi: http://dx.doi.org/10.1016/j.pain.2003.09.020

Stone, Arthur A. PhD, Broderick, Joan E. PhD, Schneider, Stefan PhD, \& Schwartz, Joseph E. PhD. (2012). Expanding Options for Developing Outcome Measures From Momentary Assessment Data. Psychosomatic Medicine, 74(4), 387-397. doi: dx.doi.org/10.1097/PSY.0b013e3182571faa

Stone, Arthur A., Schwartz, Joseph E., Neale, John M., Shiffman, Saul, Marco, Christine A., Hickcox, Mary, . . . Cruise, Laura J. (1998). A Comparison of Coping Assessed by Ecological Momentary Assessment and Retrospective Recall. Journal of Personality \& Social Psychology, 74(6), 1670-1680. doi: dx.doi.org/10.1037//00223514.74.6.1670

Strauss, A, \& Corbin, J. (1994). Grounded theory methodology Handbook of qualitative research (pp. 273-285).

Strauss, A., \& Corbin, J.M. (1997). Grounded theory in practice: Sage Publications, Incorporated.

Strauss, A.L. (1987). Qualitative analysis for social scientists: Cambridge University Press. 
Strauss, A.L., Corbin, J., Fagerhaugh, S., Glaser, B.G., Maines, D., Suczek, B., \& Wiener, CL. (1984). Chronic illness and the quality of life. St. Louis: Mosby

Strauss, Anselm L. (1998). Basics of qualitative research : techniques and procedures for developing grounded theory (2nd ed.. ed.). Thousand Oaks: Sage Publications.

Strong, J., \& Large, R. (1995). Coping with Chronic Low Back Pain: An Idiographic Exploration Through Focus Groups. The International Journal of Psychiatry in Medicine, 25(4), 371-387. doi: 10.2190/H4P9-U5NB-2KJU-4TBN

Sturgeon, J, \& Zautra, A. (2010). Resilience: A New Paradigm for Adaptation to Chronic Pain. Current Pain and Headache Reports, 14(2), 105-112. doi: dx.doi.org/10.1007/s11916-010-0095-9

Sullivan, M. J., Martel, M. O., Tripp, D. A., Savard, A., Crombez, G., \& Sullivan, M. J. L. (2006). Catastrophic thinking and heightened perception of pain in others. Pain, 123(1-2), 37-44. doi: doi:10.1016/j.pain.2006.02.007

Sullivan, M. J., Thorn, B., Haythornthwaite, J. A., Keefe, F., Martin, M., Bradley, L. A., \& Lefebvre, J. C. (2001). Theoretical perspectives on the relation between catastrophizing and pain. Clinical Journal of Pain, 17(1), 52-64. doi: dx.doi.org/10.1097/00002508-200103000-00008

Sullivan, Mark. (2004). Exaggerated pain behavior: by what standard? Clinical Journal of Pain, 20(6), 433-439. doi: dx.doi.org/10.1097/00002508-200411000-00008

Sullivan, Michael J. (2008). Toward a Biopsychomotor Conceptualization of Pain: Implications for Research and Intervention. Clinical Journal of Pain, 24(4), 281-290

doi: 10.1097/AJP.0b013e318164bb15

Sullivan, Michael J. L. PhD, Adams, Heather, \& Ellis, Tamra. (2012). Targeting Catastrophic Thinking to Promote Return to Work in Individuals With Fibromyalgia. Journal of Cognitive Psychotherapy, 26(2), 130-142. doi: Doi: 10.1016/0272-7358(88)90050-5

10.1146/annurev.psych.55.090902.142015

Sullivan, Michael JL, Bishop, Scott R, \& Pivik, Jayne. (1995). The pain catastrophizing scale: Development and validation. Psychological Assessment, 7(4), 524. doi: dx.doi.org/10.1037//1040-3590.7.4.524

Sullivan, Michael, Tanzer, Michael, Stanish, William, Fallaha, Michel, Keefe, Francis J., Simmonds, Maureen, \& Dunbar, Michael. (2009). Psychological determinants of problematic outcomes following total knee arthroplasty. Pain, 143(1-2), 123-129. doi: doi:10.1016/j.pain.2009.02.011

Sutherland, Ruth, \& Morley, Stephen. (2008). Self-pain enmeshment: Future possible selves, sociotropy, autonomy and adjustment to chronic pain. Pain, 137(2), 366-377. doi: http://dx.doi.org/10.1016/j.pain.2007.09.023

Sutton, R.I., \& Staw, B.M. (1995). What theory is not. Administrative science quarterly, 40(3), 371-384. doi: dx.doi.org/10.2307/2393788 
Tan, G., Jensen, M. P., Robinson-Whelen, S., Thornby, J. I., \& Monga, T. N. (2001). Coping with chronic pain: a comparison of two measures. Pain, 90(1-2), 127-133. doi: dx.doi.org/10.1016/S0304-3959(00)00395-X

Tang, Nicole K., Goodchild, Claire E., Hester, Joan, \& Salkovskis, Paul M. (2010). Mental defeat is linked to interference, distress and disability in chronic pain. Pain, 149(3), 547-554. doi: http://dx.doi.org/10.1016/j.pain.2010.03.028

Tennen, Howard, Affleck, Glenn, Armeli, Stephen, \& Carney, Margaret Anne. (2000). A daily process approach to coping: Linking theory, research, and practice. American Psychologist, 55(6), 626-636. doi: http://dx.doi.org/10.1037/0003-066X.55.6.626

Thagard, Paul R. (1978). The best explanation: Criteria for theory choice. The Journal of Philosophy, 75(2), 76-92. doi: dx.doi.org/10.2307/2025686

Thernstrom, M. (2010). The Pain Chronicles: Cures, myths, mysteries, prayers, diaries, brain scans, healing, and the science of suffering. New York: Farrar, Straus and Giraux.

Thomas, Elaine, Peat, George, Harris, Lindsey, Wilkie, Ross, \& Croft, Peter R. (2004). The prevalence of pain and pain interference in a general population of older adults: crosssectional findings from the North Staffordshire Osteoarthritis Project (NorStOP). Pain, 110(1-2), 361-368. doi: dx.doi.org/10.1016/S0304-3959(04)00207-6

Thomas, G., \& James, D. (2006). Reinventing grounded theory: Some questions about theory, ground and discovery. British Educational Research Journal, 32(6), 767-795. doi: dx.doi.org/10.1080/01411920600989412

Thompson, B. (2012). Are you ready? Readiness to return to work for people living with chronic pain. OT Now, 14(5), 13-15.

Thompson, Miles, \& McCracken, Lance M. (2011). Acceptance and related processes in adjustment to chronic pain. Current Pain \& Headache Reports, 15(2), 144-151. doi: dx.doi.org/10.1007/s11916-010-0170-2

Throop, C. J. (2008). From pain to virtue: dysphoric sensations and moral sensibilities in Yap (Waqab), Federated States of Micronesia. Transcultural Psychiatry, 45(2), 253-286. doi: dx.doi.org/10.1177/1363461508089767

Thulesius, Hans. (2011). A Commentary on Ekins (2011). The Grounded Theory Review: An international journal, 10(3), 55.

Tracey, I., \& Bushnell, M. C. (2009). How neuroimaging studies have challenged us to rethink: is chronic pain a disease? Journal of Pain, 10(11), 1113-1120. doi: dx.doi.org/10.1016/j.jpain.2009.09.001

Tracey, Irene, \& Mantyh, Patrick W. (2007). The cerebral signature for pain perception and its modulation. Neuron, 55(3), 377-392. doi: DOI 10.1016/j.neuron.2007.07.012

Trudeau, J., Turk, D., Dworkin, R., Benson, C., Biondi, D., Kim, M., . . Katz, N. (2012). Validation of the revised short form McGill Pain Questionnaire (SF-MPQ-2) for selfreport of pain qualities in patients with acute low back pain. The Journal of Pain, 13(4, Supplement), S4. doi: http://dx.doi.org/10.1016/j.jpain.2012.01.022 
Turk, D C, \& Monarch, E S. (2002). Biopsychosocial perspective on chronic pain. In D. C. Turk \& R. J. Gatchel (Eds.), Psychological approaches to pain management (2 ed., pp. 3 - 29). New York: The Guilford Press.

Turk, D. C., Dworkin, R. H., Allen, R. R., Bellamy, N., Brandenburg, N., Carr, D. B., . . . Turk, Dennis C. (2003). Core outcome domains for chronic pain clinical trials: IMMPACT recommendations. Pain, 106(3), 337-345. doi: dx.doi.org/10.1016/j.pain.2003.08.001

Turk, D. C., Dworkin, R. H., Burke, L. B., Gershon, R., Rothman, M., Scott, J., .. . Wyrwich, K. W. (2006). Developing patient-reported outcome measures for pain clinical trials: IMMPACT recommendations. Pain, 125(3), 208-215. doi: dx.doi.org/10.1016/j.pain.2006.09.028

Turk, D. C., \& Okifuji, A. (2002). Psychological factors in chronic pain: evolution and revolution. Journal of Consulting \& Clinical Psychology, 70(3), 678-690. doi: dx.doi.org/10.1037//0022-006X.70.3.678

Turk, D. C., \& Rudy, T. E. (1990). Neglected factors in chronic pain treatment outcome studies--referral patterns, failure to enter treatment, and attrition. Pain, 43(1), 7-25. doi: dx.doi.org/10.1016/0304-3959(90)90046-G

Turk, D. C., \& Rudy, T. E. (1991). Neglected topics in the treatment of chronic pain patients-relapse, noncompliance, and adherence enhancement. Pain, 44(1), 5-28. doi: dx.doi.org/10.1016/0304-3959(91)90142-K

Turk, D. C., Rudy, T. E., \& Sorkin, B. A. (1993). Neglected topics in chronic pain treatment outcome studies: determination of success. Pain, 53(1), 3-16. doi: dx.doi.org/10.1016/0304-3959(93)90049-U

Turk, D.C. (2002). A cognitive-behavioral perspective on treatment of chronic pain patients. In D. C. Turk \& D. C. Gatchel (Eds.), Psychological approaches to pain management: A practitioner's handbook (2 ed., pp. 138-158). New York, NY: Guilford Publications.

Turk, Dennis C, \& Wilson, Hilary D. (2010). Fear of pain as a prognostic factor in chronic pain: conceptual models, assessment, and treatment implications. Current Pain and Headache Reports, 14(2), 88-95. doi: doi:10.1007/s11916-010-0094-x.

Turk, Dennis C., Wilson, Hilary D., \& Cahana, Alex. (2011). Pain 2: Treatment of chronic non-cancer pain. The Lancet, 377(9784), 2226-2235. doi: 10.111/j.14681331.2010.02999.x

Turnbull, Sharon. (2002). Social Construction Research and Theory Building. Advances in Developing Human Resources, 4(3), 317-334. doi: 10.1177/1523422302043006

Turner, Judith A., Clancy, Steve, \& Vitaliano, Peter P. (1987). Relationships of stress, appraisal and coping, to chronic low back pain. Behaviour Research and Therapy, 25(4), 281-288. doi: http://dx.doi.org/10.1016/0005-7967\%2887\%2990006-4

Twohig, Michael P. (2012). Acceptance and commitment therapy: Introduction. Cognitive and Behavioral Practice, 19(4), 499-507. doi: 1077-7229/12/499-507\$1.00/0 
Vaillant, G. E. (2000). Adaptive mental mechanisms. Their role in a positive psychology. American Psychologist, 55(1), 89-98. doi: dx.doi.org/10.1037//0003-066X.55.1.89

Van Damme, S, Crombez, G, \& Eccleston, C. (2008). Coping with pain: A motivational perspective. Pain, 139(1), 1-4. doi: http://dx.doi.org/10.1016/j.pain.2008.07.022

Van Damme, S., Legrain, V., Vogt, J., \& Crombez, G. (2010). Keeping pain in mind: a motivational account of attention to pain. Neuroscience \& Biobehavioral Reviews, 34(2), 204-213. doi: dx.doi.org/10.1016/j.neubiorev.2009.01.005

Van Damme, Stefaan, Van Ryckeghem, Dimitri M. L., Wyffels, Fran, Van Hulle, Lore, \& Crombez, Geert. (2012). No pain no gain? Pursuing a competing goal inhibits avoidance behavior. Pain, 153(4), 800-804. doi: 10.1016/j.pain.2011.12.015

Vancleef, L. M., Peters, M. L., De Jong, P. J., Vancleef, Linda M. G., Peters, Madelon L., \& De Jong, Peter J. (2009). Interpreting ambiguous health and bodily threat: are individual differences in pain-related vulnerability constructs associated with an online negative interpretation bias? Journal of Behavior Therapy \& Experimental Psychiatry, 40(1), 59-69. doi: dx.doi.org/10.1016/j.jbtep.2008.03.004

Veehof, Martine M., Oskam, Maarten-Jan, Schreurs, Karlein M., \& Bohlmeijer, Ernst T. (2011). Acceptance-based interventions for the treatment of chronic pain: A systematic review and meta-analysis. Pain, 152(3), 533-542. doi: doi:10.1016/j.pain.2010.11.002

Vertue, F.M., \& Haig, B.D. (2008). An abductive perspective on clinical reasoning and case formulation. Journal of Clinical Psychology, 64(9), 1046-1068.

Vik, K, Nygard, L, Borell, L, \& Josephsson, S. (2008). Agency and engagement: Older adults' experiences of participation in occupation during home-based rehabilitation. Canadian Journal of Occupational Therapy, 75(5), 262-271.

Villemure, C., \& Schweinhardt, P. (2010). Supraspinal pain processing: distinct roles of emotion and attention. Neuroscientist, 16(3), 276-284. doi: DOI: $10.1177 / 1073858409359200$

Vlaeyen, J. W., De Jong, J. R., Onghena, P., Kerckhoffs-Hanssen, M., \& Kole-Snijders, A. M. (2002). Can pain-related fear be reduced? The application of cognitivebehavioural exposure in vivo. Pain Research \& Management, 7(3), 144-153.

Vlaeyen, J. W., \& Linton, S. J. (2000). Fear-avoidance and its consequences in chronic musculoskeletal pain: a state of the art. Pain., 85(3), 317-332. doi: dx.doi.org/10.1016/S0304-3959(99)00242-0

Vlaeyen, J. W., \& Linton, S. J. (2006). Are we "fear-avoidant?". Pain 124(3), 240-241. doi: dx.doi.org/10.1016/j.pain.2006.06.031

Vlaeyen, J. W., \& Linton, S. J. (2012). Fear-avoidance model of chronic musculoskeletal pain: 12 years on. Pain, 153(6), 1144-1147. doi: dx.doi.org/10.1016/j.pain.2011.12.009

Vlaeyen, J. W., Seelen, H. A., Peters, M., de Jong, P., Aretz, E., Beisiegel, E., \& Weber, W. E. (1999). Fear of movement/(re)injury and muscular reactivity in chronic low back 
pain patients: an experimental investigation. Pain., 82(3), 297-304. doi: dx.doi.org/10.1016/S0304-3959(99)00054-8

Vlaeyen, Johan, Morley, Stephen, Linton, Steven, Boersma, Katja, \& de Jong, Jeroen. (2012a). Pain-related Fear. Seattle: IASP Press.

Vlaeyen, Johan, Morley, Stephen, Linton, Steven J, Boersma, Katja, \& de Jong, Jeroen. (2012b). Pain-related fear: exposure based treatment for chronic pain.

Vlaeyen, Johan W., Kole-Snijders, Ank M., Boeren, Ruben G., \& van Eek, H. (1995). Fear of movement/(re)injury in chronic low back pain and its relation to behavioral performance. Pain, 62(3), 363-372. doi: dx.doi.org/10.1016/0304-3959(94)00279-N

Vlaeyen, Johan W., Kole-Snijders, Ank M., Rotteveel, Annemarie M., Ruesink, Renske, \& Heuts, Peter H. (1995). The role of fear of movement/(re)injury in pain disability. Journal of Occupational Rehabilitation, 5(4), 235-252. doi: dx.doi.org/10.1007/BF02109988

Vlaeyen, Johan W. S., \& Morley, Stephen. (2005). Cognitive-Behavioral Treatments for Chronic Pain: What Works for Whom? The Clinical Journal of Pain, 21(1), 1-8. doi: dx.doi.org/10.1097/00002508-200501000-00001

von Humboldt, Sofia, Leal, Isabel, \& Pimenta, Filipa. (2014). Living well in later life: The influence of sense of coherence, and socio-demographic, lifestyle and health-related factors on older adults' satisfaction with life. Applied Research in Quality of Life, 9(3), 631-642. doi: dx.doi.org/10.1007/s11482-013-9262-6

Vowles, K. E., McCracken, L. M., \& Eccleston, C. (2007a). Processes of change in treatment for chronic pain: the contributions of pain, acceptance, and catastrophizing. European Journal of Pain: Ejp, 11(7), 779-787. doi: dx.doi.org/10.1016/j.ejpain.2006.12.007

Vowles, Kevin E., \& McCracken, Lance M. (2008). Acceptance and values-based action in chronic pain: A study of treatment effectiveness and process. Journal of Consulting and Clinical Psychology, 76(3), 397-407. doi: http://dx.doi.org/10.1037/0022$\underline{006 X .76 .3 .397}$

Vowles, Kevin E., McCracken, Lance M., \& Eccleston, Christopher. (2007b). Processes of change in treatment for chronic pain: The contributions of pain, acceptance, and catastrophizing. European Journal of Pain, 11(7), 779-787. doi: http://dx.doi.org/10.1016/j.ejpain.2006.12.007

Vowles, Kevin E., McCracken, Lance M., \& O'Brien, Jane Zhao. (2011). Acceptance and values-based action in chronic pain: A three-year follow-up analysis of treatment effectiveness and process. Behaviour Research and Therapy, 49(11), 748-755. doi: dx.doi.org/10.1016/j.brat.2011.08.002

Vowles, Kevin E., \& Thompson, Miles. (2011). Acceptance and commitment therapy for chronic pain. McCracken, Lance M [Ed], 31-60.

Wacker, J.G. (1998). A definition of theory: research guidelines for different theory-building research methods in operations management. Journal of Operations Management, 16(4), 361-385. doi: dx.doi.org/10.1016/S0272-6963(98)00019-9

Waddell, G. (2004). The Back Pain Revolution (2 ed.). Edinburgh: Churchill Livingstone. 
Waddell, G., Bircher, M., Finlayson, D., \& Main, C. J. (1984). Symptoms and signs: physical disease or illness behaviour? British Medical Journal Clinical Research Ed, 289(6447), 739-741. doi: dx.doi.org/10.1136/bmj.289.6447.739

Waddell, Gordon, Main, Chris J., Morris, Emyr W., Paola, Michael D. I., \& Gray, Iain C. (1984). Chronic Low-Back Pain, Psychologic Distress, and Illness Behavior. Spine 9(2), 209-213. doi: dx.doi.org/10.1097/00007632-198403000-00013

Waddell, Gordon, Pilowsky, Issy, \& Bond, Michael R. (1989). Clinical assessment and interpretation of abnormal illness behaviour in low back pain. Pain, 39(1), 41-53. doi: http://dx.doi.org/10.1016/0304-3959(89)90174-7

Wade, D.T., \& Halligan, P.W. (2004). Do biomedical models of illness make for good healthcare systems? BMJ: British Medical Journal, 329(7479), 1398. doi: dx.doi.org/10.1136/bmj.329.7479.1398

Walker, B. F., Muller, R., \& Grant, W. D. (2004). Low back pain in Australian adults. health provider utilization and care seeking. Journal of Manipulative \& Physiological Therapeutics, 27(5), 327-335.

Walker, D., \& Myrick, F. (2006a). Grounded theory: An exploration of process and procedure. Qualitative Health Research, 16(4), 547-559. doi: http://dx.doi.org/10.1177/1049732305285972

Walker, Diane, \& Myrick, Florence. (2006b). Grounded Theory: An Exploration of Process and Procedure. Qualitative Health Research, 16(4), 547 - 559. doi: http://dx.doi.org/10.1177/1049732305285972

Walker, Jan, Sofaer, Beatrice, \& Holloway, Immy. (2006). The experience of chronic back pain: Accounts of loss in those seeking help from pain clinics. European Journal of Pain, 10(3), 199-207. doi: http://dx.doi.org/10.1016/j.ejpain.2005.03.007

Ward, Tony, \& Haig, Brian. (1997). Abductive reasoning and clinical assessment. Australian Psychologist, 32(2), 93-100. doi: dx.doi.org/10.1080/00050069708257360

Ward, Tony, \& Hudson, Stephen M. (1998). The construction and development of theory in the sexual offending area: A metatheoretical framework. Sexual Abuse: Journal of Research and Treatment, 10(1), 47-63. doi: dx.doi.org/10.1177/107906329801000106

Warren, John C. (1829). Cases of Neuralgia, or Painful Affections of Nerves. The Boston Medical and Surgical Journal, 2(9), 129-135. doi: doi:10.1056/NEJM182904140020901

Watson, Jeanne C, \& Greenberg, Leslie S. (2009). Empathic Resonance: A Neuroscience Perspective. In J. Decety \& W. Ickes (Eds.), The social neuroscience of empathy (pp. 125-137). Massachusetts: MIT.

Watson, N. (2002). Well, I know this is going to sound very strange to you, but I don't see myself as a disabled person: Identity and disability. Disability \& Society, 17(5), 509527. doi: dx.doi.org/10.1080/09687590220148496

Watson, P. (2000). Techniques for the increase of physical activity. In C. Main \& C.

Spanswick (Eds.), Pain Management: An interdisciplinary approach (pp. 285-296).

Edinburgh: Churchill Livingstone. 
Weed, Mike. (2009). Research quality considerations for grounded theory research in sport \& exercise psychology. Psychology of Sport and Exercise, 10(5), 502-510. doi: http://dx.doi.org/10.1016/j.psychsport.2009.02.007

Wegener, Stephen T., Castillo, Renan C., Haythornthwaite, Jennifer, MacKenzie, Ellen J., \& Bosse, Michael J. (2011). Psychological distress mediates the effect of pain on function. Pain, 152(6), 1349-1357. doi: dx.doi.org/10.1016/j.pain.2011.02.020

Wein, Simon. (2011). Impact of culture on the expression of pain and suffering. Journal of Pediatric Hematology/Oncology, 33 (Suppl 2), S105-107. doi: dx.doi.org/10.1097/MPH.0b013e318230dd98

Weiss, Karen E., Hahn, Amy, Wallace, Dustin P., Biggs, Bridget, Bruce, Barbara K., \& Harrison, Tracy E. (2013). Acceptance of pain: Associations with depression, catastrophizing, and functional disability among children and adolescents in an interdisciplinary chronic pain rehabilitation program. Journal of Pediatric Psychology, 38(7), 756-765. doi: dx.doi.org/10.1093/jpepsy/jst028

Westman, Anders E., Boersma, Katja, Leppert, Jerzy, \& Linton, Steven J. (2011). Fearavoidance beliefs, catastrophizing, and distress: A longitudinal subgroup analysis on patients with musculoskeletal pain. The Clinical Journal of Pain, 27(7), 567-577. doi: dx.doi.org/10.1097/AJP.0b013e318219ab6c

Wetherell, Julie Loebach, Afari, Niloofar, Rutledge, Thomas, Sorrell, John T., Stoddard, Jill A., Petkus, Andrew J., . . . Hampton Atkinson, J. (2011). A randomized, controlled trial of acceptance and commitment therapy and cognitive-behavioral therapy for chronic pain. Pain, 152(9), 2098-2107. doi: http://dx.doi.org/10.1016/j.pain.2011.05.016

WFOT. (2010, 2011). Definition "occupation". Retrieved 05/09/2013, 2013, from http://www.wfot.org/aboutus/aboutoccupationaltherapy/definitionofoccupationalthera py.aspx

WHO, World Health Organisation. (1980). The International Classification of Impairments, Disabilities and Handicaps (ICIDH). Geneva: World Health Organisation.

Wicksell, R. K., Ahlqvist, J., Bring, A., Melin, L., \& Olsson, G. L. (2008). Can exposure and acceptance strategies improve functioning and life satisfaction in people with chronic pain and whiplash-associated disorders (WAD)? A randomized controlled trial. Cognitive Behaviour Therapy, 37(3), 169-182. doi: dx.doi.org/10.1080/16506070802078970

Wideman, Timothy H, Asmundson, GG, Smeets, RJ, Zautra, Alex J, Simmonds, Maureen J, Sullivan, MJ, . . . Edwards, Robert R. (2013). Re-Thinking the Fear Avoidance Model: Toward a Multi-Dimensional Framework of Pain-Related Disability. Pain. doi: dx.doi.org/10.1016/j.pain.2013.06.005

Wiech, K., Ploner, M., \& Tracey, I. (2008). Neurocognitive aspects of pain perception. Trends in Cognitive Sciences, 12(8), 306-313. doi: doi:10.1016/j.tics.2008.05.005

Wiech, K., \& Tracey, I. (2009). The influence of negative emotions on pain: behavioral effects and neural mechanisms. Neuroimage, 47(3), 987-994. doi: dx.doi.org/10.1016/j.neuroimage.2009.05.059 
Wilcock, A. (1993). Keynote paper: biological and sociocultural aspects of occupation, health and health promotion. British Journal of Occupational Therapy, 56(6), 200-203.

Williams, Amanda C. de C., Eccleston, Christopher, \& Morley, Stephen. (2012).

Psychological therapies for the management of chronic pain (excluding headache) in adults. Cochrane Database of Systematic Reviews, (11). http://onlinelibrary.wiley.com/doi/10.1002/14651858.CD007407.pub3/abstract doi:10.1002/14651858.CD007407.pub3

Williams, Elizabeth Nutt, \& Morrow, Susan L. (2009). Achieving trustworthiness in qualitative research: A pan-paradigmatic perspective. Psychotherapy Research, 19(45), 576-582. doi: 10.1080/10503300802702113

Wilson, Sarah. (2007). 'When you have children, you're obliged to live': Motherhood, chronic illness and biographical disruption. Sociology of Health \& Illness, 29(4), 610-626. doi: 10.1111/j.1467-9566.2007.01008.x

Wiltshier, Fiona. (2011). Researching With NVivo. Forum Qualitative Sozialforschung / Forum: Qualitative Social Research, 12(1). http://www.qualitativeresearch.net/index.php/fqs/article/view/1628

Witvrouw, E., Pattyn, E., Almqvist, K. F., Crombez, G., Accoe, C., Cambier, D., . . Witvrouw, Erik. (2009). Catastrophic thinking about pain as a predictor of length of hospital stay after total knee arthroplasty: a prospective study. Knee Surgery, Sports Traumatology, Arthroscopy, 17(10), 1189-1194. doi: dx.doi.org/10.1007/s00167-0090817-x

Woolcock, H, Thearle, M J, \& Saunders, K. (1997). 'My Beloved Chloroform'. Attitudes to Childbearing in Colonial Queensland: A Case Study. Social History of Medicine, 10(3), 437-457. doi: 10.1093/shm/10.3.437

Woolf, Clifford J. (2010). Overcoming obstacles to developing new analgesics. Nature Medical, 16(11), 1241-1247. doi: doi:10.1038/nm.2230

Wright, Andrew. (2011). A Criticism of the IASPs Definition of Pain. Journal of Consciousness Studies, 18(9-10), 19-44.

Yeung, Ellen Wanheung, Arewasikporn, Anne, \& Zautra, Alex J. (2012). Resilience and chronic pain. Journal of Social and Clinical Psychology, 31(6), 593-617. doi: http://dx.doi.org/10.1521/jscp.2012.31.6.593

Yoshida, Toshiyuki, Molton, Ivan R., Jensen, Mark P., Nakamura, Tomoyasu, Arimura, Tatsuyuki, Kubo, Chiharu, \& Hosoi, Masako. (2012). Cognitions, metacognitions, and chronic pain. Rehabilitation Psychology, 57(3), 207-213. doi: dx.doi.org/10.1037/a0028903

Zautra, A. J., Hamilton, N., \& Yocum, D. (2000). Patterns of positive social engagement among women with rheumatoid arthritis... Habits I Conference [January 1999]. Occupational Therapy Journal of Research, 20, 21S-40s.

Zautra, Alex J., Arewasikporn, Anne, \& Davis, Mary C. (2010). Resilience: Promoting wellbeing through recovery, sustainability, and growth. Research in Human Development, 7(3), 221-238. doi: http://dx.doi.org/10.1080/15427609.2010.504431 
Zautra, Alex J., Johnson, Lisa M., \& Davis, Mary C. (2005). Positive Affect as a Source of Resilience for Women in Chronic Pain. Journal of Consulting and Clinical Psychology, 73(2), 212-220. doi: dx.doi.org/10.1037/0022-006X.73.2.212

Zaza, C., \& Baine, N. (2002). Cancer pain and psychosocial factors: a critical review of the literature. Journal of Pain \& Symptom Management, 24(5), 526-542.

Zeidan, Fadel, Martucci, Katherine T., Kraft, Robert A., Gordon, Nakia S., McHaffie, John G., \& Coghill, Robert C. (2011). Brain mechanisms supporting the modulation of pain by mindfulness meditation. The Journal of Neuroscience, 31(14), 5540-5548. doi: dx.doi.org/10.1523/JNEUROSCI.5791-10.2011

Zikmund-Fisher, Brian J., Couper, Mick P., Singer, Eleanor, Ubel, Peter A., Ziniel, Sonja, Fowler, Floyd J., Jr., . . . Fagerlin, Angela. (2010). Deficits and variations in patients' experience with making 9 common medical decisions: The DECISIONS survey. Medical Decision Making, 30(5, Suppl), 85S-95S. doi: DOI: 10.1177/0272989X10380466

Zimmermann, Harm-Peer, \& Grebe, Heinrich. (2014). "Senior coolness": Living well as an attitude in later life. Journal of Aging Studies, 28, 22-34. doi: http://dx.doi.org/10.1016/j.jaging.2013.11.002

Zvolensky, Michael J., Feldner, Matthew T., Eifert, Georg H., Vujanovic, Anka A., \& Solomon, Sondra E. (2008). Cardiophobia: A Critical Analysis. Transcultural Psychiatry, 45(2), 230-252. doi: 10.1177/1363461508089766 


\section{Appendix 1: Ethics}

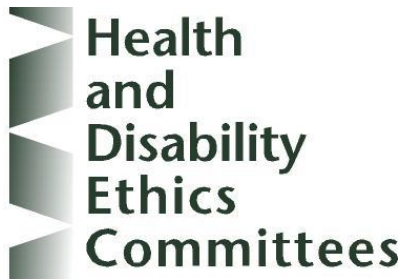

\author{
Upper South B Regional Ethics Committee \\ c/- Ministry of Health \\ 6 Hazeldean Road, Level 1 Montgomery Watson Building \\ Addington, Christchurch \\ Phone: (03) 9742305 \\ Email: uppersouthb_ethicscommittee@moh.govt.nz
}

16 August 2011

Ms Bronwyn Thompson

9 Gresham Terrace

North New Brighton

Christchurch 8083

Dear Ms Thompson

Re: Ethics ref: URB/11/02/009 (please quote in all correspondence)

Study title: Living Well with Persistent Pain: A Grounded Theory Study

Investigators: Ms Bronwyn Thompson

This study was given ethical approval by the Upper South B Regional Ethics Committee on 16 August 2011. A list of members of the Committee is attached.

\section{Approved Documents}

— Information Sheet and Consent Form version dated 24 July 2011

This approval is valid until 30 June 2013, provided that Annual Progress Reports are submitted (see below). 


\section{Amendments and Protocol Deviations}

All significant amendments to this proposal must receive prior approval from the Committee. Significant amendments include (but are not limited to) changes to:

- the researcher responsible for the conduct of the study at a study site

- the addition of an extra study site

— the design or duration of the study

— the method of recruitment

— information sheets and informed consent procedures.

Significant deviations from the approved protocol must be reported to the Committee as soon as possible.

\section{Annual Progress Reports and Final Reports}

The first Annual Progress Report for this study is due to the Committee by 31 August 2012. The Annual Report Form that should be used is available at www.ethicscommittees. health.govt.nz. Please note that if you do not provide a progress report by this date, ethical approval may be withdrawn.

A Final Report is also required at the conclusion of the study. The Final Report Form is also available at www.ethicscommittees.health.govt.nz.

\section{Statement of compliance}

The committee is constituted in accordance with its Terms of Reference. It complies with the Operational Standard for Ethics Committees and the principles of international good clinical practice.

The committee is approved by the Health Research Council's Ethics Committee for the purposes of section 25(1)(c) of the Health Research Council Act 1990.

We wish you all the best with your study.

Yours sincerely

$$
\text { Diana } 7 \text {. Whipp }
$$

Mrs Diana Whipp

\section{Administrator Upper South B Regional Ethics Committee}

Email: uppersouthb_ethicscommittee@moh.govt.nz 


\section{Health Sciences Centre}

Associate Professor Ray Kirk - Director

\section{Statement of Consent}

\section{Living Well with Persistent Pain: A Grounded Theory Study}

Principal Investigator

Bronwyn Thompson

\section{Request for Interpreter}

\begin{tabular}{|c|c|c|}
\hline English & I wish to have an interpreter. & Yes No \\
\hline Maori & $\begin{array}{l}\text { E hiahia ana ahau ki tetahi } \\
\text { kaiwhakamaori/kaiwhaka pakeha korero. }\end{array}$ & Ae Kao \\
\hline $\begin{array}{l}\text { Cook } \\
\text { Island }\end{array}$ & Ka inangaro au i tetai tangata uri reo. & Ae Kare \\
\hline Fijian & $\begin{array}{l}\text { Au gadreva me dua e vakadewa vosa vei } \\
\text { au }\end{array}$ & lo Sega \\
\hline Niuean & $\begin{array}{l}\text { Fia manako au ke fakaaoga e taha tagata } \\
\text { fakahokohoko kupu. }\end{array}$ & E Nakai \\
\hline Samoan & Ou te mana'o ia i ai se fa'amatala upu. & loe Leai \\
\hline Tokelaun & $\begin{array}{l}\text { Ko au e fofou ki he tino ke fakaliliu te } \\
\text { gagana Peletania ki na gagana } \\
\text { o na motu o te Pahefika }\end{array}$ & loe Leai \\
\hline Tongan & Oku ou fiema'u ha fakatonulea. & lo Ikai \\
\hline
\end{tabular}


Participant's Name

I have read and I understand the information sheet dated for people taking part in this study designed about the ways people living in the community with ongoing pain carry out important activities. I have had the opportunity to think about and discuss this study. I am satisfied with the answers I have been given.

I understand that taking part in this study is voluntary and that I may withdraw from the study at any time if I wish. This will not affect my continuing health care.

I understand that my participation in this study is confidential and that no material which could identify me will be used in any reports on this study. I understand that if I withdraw from the study, all the information I have provided will also be withdrawn.

I know whom to contact if I experience any concerns about the study or if anything occurs which I think is a reason to withdraw from the study.

This study has been given ethical approval from both the Upper South B Regional Ethics Committee, ethics reference number URB/11/02/009, and the University of Canterbury Human Ethics Committee. This means that the Committee may check at any time that the study is following appropriate ethical procedures.

I agree to my interview being recorded. YES/NO

I agree to my GP or other current provider being informed of my participation in this study. YES/NO

I would like the researcher to discuss the outcomes of the study with me. YES/NO

I have been given an opportunity to review my recording. YES/NO

I ____________________________ipant) having been fully informed about this study agree to take part in it.

Signed: Date 
Printed Name:

Address:

Signed: Date

Bronwyn Thompson, ph 388 3868, Principal Researcher, PhD candidate, Health Sciences Centre, University of Canterbury

Project explained by:

Signature

Date

Unlversity of Canterbury Private Bag 4800, Christchurch 8140, New Zealand. www.canterbury.ac.nz 


\section{Health Sciences Centre}

Associate Professor Ray Kirk - Director

Tel: +6433667001 ext. 8691, Fax: +6433643318

www.health.canterbury.ac.nz healthsciences(a)canterbury.ac.nz

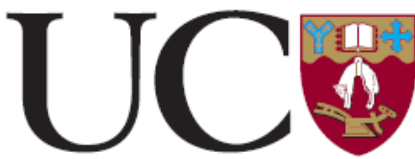

UNIVERSITY OF CANTERBURY

Te Whare Wānanga o Waitaha CHRISTCHURCH NEW ZEALAND

\section{Information sheet for participants}

\section{Living Well with Persistent Pain: A Grounded Theory Study}

Principal Investigator

Bronwyn Thompson

Although researchers know a lot of information about people who have persistent pain and ask for treatment, less is known about people who live well in spite of their ongoing pain. This study asks people about the ways they get around their pain if they have either ankylosing spondylitis or rheumatoid arthritis and think of themselves as well. This is an important topic because there is very little information about practical ways people who live well with their disease carry out important activities.

\section{What does the study involve?}

A 60 - 90 minute informal interview talking about how you set about doing important activities in your life despite your pain. The interview may be recorded and will be analysed later.

You will also be asked to complete four short questionnaires, along with some personal information such as your age and ethnicity.

You don't have to answer all of the questions in either the interview or questionnaires, and you can stop or withdraw from the study at any time. If you do decide to withdraw from the study, any information you may have provided will also be removed.

\section{What sort of questions will be asked?}

The questions that may be asked will include how you found out about your health condition, how you go about doing important activities like work, what you do during a flare-up of your condition and so on.

\section{What will the information from this study be used for?}

This study will help clinicians advising people with persistent pain to recommend ways of coping with the problem that have worked for people like you. By learning about how you developed your ways around your pain, I hope to learn how to show other people to develop similar strategies. 


\section{Who is included in the study?}

Anyone who has been diagnosed as having ankylosing spondylitis or rheumatoid arthritis, is working (in paid or unpaid work), and is not having any treatment apart from arthritis medication (eg anti-inflammatories, disease modifying drugs) is able to be part of this study.

\section{How will my details be kept private?}

None of your personal details that could identify you personally will be used in the study. The recordings will be made digitally, and kept in a secure digital format in a secure office. The recordings will be analysed and all personal details will be removed. You are welcome to ask for your recording to be returned to you. Your questionnaire responses will also be kept on a computer file, and in a secure office, and will have no personal details identifying you recorded on them. This information will be stored for 10 years and stored according to the requirements of the Health Information Privacy Code (2008).

\section{Can I bring a support person or whanau?}

Yes, it can be helpful to have someone else present during the interview. It is important, however, that your opinion is the one that is recorded.

\section{Are there any risks?}

There should be no risks from being part of this study. Some people may find it difficult or troubling to talk about how pain has affected their lives, and if this is a problem, the researcher will offer to contact support services such as GP, Maori Health Worker or Arthritis New Zealand educator.

\section{Where can I go to for more information, or to find out the results of this study?}

More information about this study is available from me (details below). A summary of final results will be made available to you at the completion of the study.

This study is being conducted as part of a PhD thesis through Health Sciences Centre, University of Canterbury. The study supervisor is Dr Jeffrey Gage, Health Sciences Centre, University of Canterbury, Private Bag 4800, Christchurch, ph 033667001 . A PhD is a public document that can be accessed via the University of Canterbury library database.

Please feel free to contact the researcher if you have any questions about this study.

If you have any questions or concerns about your rights as a participant in this research study you can contact an independent health and disability advocate. This is a free service provided under the Health and Disability Commissioner Act.

Telephone: (NZ wide) 0800555050

Free Fax (NZ wide): 080027877678 (O800 2 SUPPORT) 
Email (NZ wide): advocacy@hdc.org.nz

Bronwyn Thompson, MSc (Psych), DipOT, PhD Candidate

$\mathrm{Ph}$ (03) 3883868 (after hours, during the day you may leave a message)

Cellphone 0272704225

Email: musculo@clear.net.nz

This study has been given ethical approval Upper South B Regional Ethics Committee, ethics reference number URB/11/02/009 and Canterbury University Human Ethics Committee. This means that the Committee may check at any time that the study is following appropriate ethical procedures. 


\section{Appendix 2: Semi-structured interview}

\section{Indicative Questions}

Note: These questions are from Charmaz (2006). Many of these questions were not asked, additional questions were added, depending upon theoretical sampling, and to maximize representation and systematic exploration of topics (data). The original questions below were included in the Ethics Application, to satisfy the requirements for Ethical approval.

The opening questions used after the first two interviews were conducted were:

"How do you do the important things you want to while you have chronic pain?"

"How do you get on with life despite your pain?"

I then referred to domains emerging from the data and coding, as I recognised this was more effective at "instilling the spill."

\section{Opening questions}

1. Tell me about what happens...

2. When did you first notice/experience [your pain...]

3. What was it like? What did you think then? How did you find out you had ...? [when did you first seek medical treatment of your pain?]

4. Did anyone influence your action [to seek medical help]? Tell me about how this changed you...

5. Could you describe the events that led up to [you seeking health care....]

6. What contributed to...

7. What was going on in your life then? How would you describe how you viewed [coping with pain]... before then? How has your view changed?

8. How would you describe the person you were then?

\section{Intermediate questions}

1. What, if anything, did you know about [people having pain or disability] chronic pain before you developed your problem?

2. Tell me about your thoughts and feelings when you learned about your diagnosis. What happened next?

3. Who was involved? When was that? How were they involved?

4. Tell me how you have learned to handle your pain...What have you learned to do?

5. When did you first learn to do these things? Can you remember a time when you didn't do them?

6. What have been the most helpful things you've done to manage your pain?

7. Have there been things that you've done that haven't been unhelpful? How were they unhelpful?

8. How have your thoughts and feelings changed about... since...

9. What positive changes have occurred in your life... 
10. What not so good changes have occurred in your life...

11. Could you describe for me a typical day... And when you are sore? What are the main differences for you?

12. Tell me how you go about [doing an activity that is really important...]What do you do on a good day? What do you do on a not so good day? Is there any difference about how you go about doing things on a good or not so good day?

13. Tell me how you would describe the person you are now? What most contributed to this change/continuity?

14. As you look back on the achievements in your life since you found out you have [diagnosis]... are there any other events that stand out in your mind? Could you describe ...? How did this affect what you did? How did you respond to challenges that have got in your way?

15. Could you describe the most important things you have learned through experiencing pain?

16. Where do you see yourself in $2,5,10,20$ years from now? Describe what you hope to be doing then. How would you compare the person you hope to be and the person you see yourself as now? How will you go about getting there? What strategies will you use? [give examples?]

17. What helps you to manage...What problems might you encounter? Tell me the sources and solutions of these problems...

18. Who has been the most helpful? How has s/he been helpful?

19. Has any organization been helpful? What did they help you with? How has it been helpful?

20. Have there been any other resources you've used? What are they? How have they been helpful?

21. Have there been any strategies that you've found out about that haven't been helpful? When are they unhelpful?

\section{Ending questions}

1. What do you think are the most important ways to...How did you discover them? How has your experience before pain affected how you handled this?

2. Tell me about how your views and actions may have changed since you have had your pain problem?

3. How have you grown as a person since? Tell me about your strengths that you discovered or developed through pain? What do you most value about yourself now? What do others most value in you?

4. After having these experiences, what advice would you give to someone who has just discovered that she or he has pain?

5. Is there anything that you might not have thought about before that occurred to you during this interview?

6. Is there anything else you think I should know to understand pain better?

7. Is there anything you would like to ask me? 


\section{Appendix 3: Participants}

Although classical grounded theory tenets point to the data obtained from participants as simply data, analysed to an abstract and conceptual level, the following information is provided to assist those who would wish to compare participant characteristics with other populations. To protect participant identities, the broad industry group in which the individual works is used rather than the specific occupational group. 


\begin{tabular}{|c|c|c|c|c|c|}
\hline Study Number & 1 & 2 & 3 & 4 & 5 \\
\hline Age & 29 & 35 & 64 & 49 & 45 \\
\hline Gender & female & male & male & female & female \\
\hline Relationship status & single & married & married & single & married \\
\hline Dependents at home & 0 & 1 & 0 & 1 & 0 \\
\hline Employment status - hours, paid study & $\begin{array}{l}\text { Full time primary } \\
\text { education }\end{array}$ & $\begin{array}{l}\text { Full time other social assistance } \\
\text { services }\end{array}$ & Full time road freight transport & Full time professional technical services & $\begin{array}{l}\text { Full time self-employed sports and } \\
\text { recreation instruction }\end{array}$ \\
\hline Ethnicity & NZ European & NZ European & NZ European & Other European & NZ Maori \\
\hline Self-reported diagnosis & rheumatoid arthritis & psoriatic arthritis & osteoarthritis hips, knees & hypermobility syndrome & rheumatoid arthritis, fibromyalgia \\
\hline \multicolumn{6}{|l|}{ Pain Activities Relation Questionnaire } \\
\hline Contingency & 4.14 & 4.14 & 4.86 & 3.71 & 3.14 \\
\hline Avoidance & 2.13 & 1.88 & 4.75 & 2 & 2.63 \\
\hline Pacing & 2.83 & 4.67 & 4.33 & 4 & 3.17 \\
\hline Confronting & 3.29 & 3.43 & 3.43 & 3 & 3.71 \\
\hline \multicolumn{6}{|l|}{ Chronic Pain Acceptance Questionnaire } \\
\hline Willingness & 14 & 15 & 17 & 17 & 16 \\
\hline Activity engagement & 23 & 20 & 19 & 18 & 22 \\
\hline \multicolumn{6}{|l|}{ Chronic Pain Coping Inventory } \\
\hline Guarding & 12.00 & 12.14 & 3.00 & 13.14 & 12.29 \\
\hline Resting & 8.40 & 10.40 & 2.00 & 10.40 & 7.40 \\
\hline Asking for assistance & 14.75 & 14.50 & 17.50 & 9.00 & 10.25 \\
\hline Exercise/stretch & 19.00 & 20.20 & 21.20 & 19.00 & 18.00 \\
\hline Relaxation & 24.20 & 24.20 & 25.20 & 25.20 & 21.20 \\
\hline Task persistence & 25.00 & 24.00 & 25.00 & 23.67 & 23.83 \\
\hline Coping self-statements & 11.60 & 11.60 & 10.60 & 12.60 & 11.40 \\
\hline Seeking social support & 18.60 & 17.60 & 23.20 & 20.00 & 21.00 \\
\hline \multicolumn{6}{|l|}{ Profile of Chronic Pain - Screen } \\
\hline Severity & 23.00 & 19.00 & 24.00 & 24.00 & 20.00 \\
\hline Interference & 6.00 & 9.00 & 5.00 & 7.00 & 6.00 \\
\hline Emotional burden & 5.00 & 7.00 & 7.00 & 2.00 & 5.00 \\
\hline
\end{tabular}


Thompson, B. Living Well with Chronic Pain: A grounded theory Appendix 3 - Participants

\begin{tabular}{|c|c|c|c|c|c|}
\hline Study Number & 6 & 7 & 8 & 9 & 10 \\
\hline Age & 26 & 58 & 35 & 67 & 52 \\
\hline Gender & male & female & female & female & male \\
\hline Relationship status & single & married & married & married & defacto \\
\hline Employment status - hours, paid/unpaid/study & $\begin{array}{c}\text { Full time agriculture and fishing } \\
\text { support services }\end{array}$ & Full time higher education & Full time hospitals & Full time hospitals & Full time laboratory services \\
\hline Ethnicity & NZ European & English & NZ European & NZ European & NZ European \\
\hline Contingency & 2.14 & 3 & 4 & 4.14 & 4.43 \\
\hline Avoidance & 1.13 & 3 & 3.88 & 4.13 & 2.5 \\
\hline Pacing & 3.83 & 3.5 & 4.33 & 1.67 & 3 \\
\hline Confronting & 1.71 & 3.14 & 2.71 & 3 & 3.86 \\
\hline \multicolumn{6}{|l|}{ Chronic Pain Acceptance Questionnaire } \\
\hline Willingness & 21 & 20 & 19 & 16 & 24 \\
\hline Asking for assistance & 9.00 & 14.50 & 11.25 & 14.50 & 8.50 \\
\hline Exercise/stretch & 21.20 & 23.80 & 22.20 & 23.80 & 19.00 \\
\hline Relaxation & 24.20 & 18.00 & 22.20 & 24.20 & 21.20 \\
\hline Task persistence & 25.00 & 23.67 & 26.00 & 23.83 & 27.00 \\
\hline Coping self-statements & 11.60 & 10.60 & 12.60 & 11.60 & 15.60 \\
\hline Seeking social support & 17.60 & 20.00 & 21.20 & 23.20 & 25.00 \\
\hline \multicolumn{6}{|l|}{ Profile of Chronic Pain - Screen } \\
\hline Severity & 17.00 & 20.00 & 19.00 & 22.00 & 16.00 \\
\hline Interference & 4.00 & 0.00 & 0.00 & 1.00 & 3.00 \\
\hline Emotional burden & 6.00 & 2.00 & 0.00 & 1.00 & 3.00 \\
\hline
\end{tabular}




\begin{tabular}{|c|c|c|c|c|c|}
\hline Study Number & 11 & 12 & 13 & 14 & 15 \\
\hline Age & 48 & 32 & 55 & 50 & 42 \\
\hline Gender & female & male & male & male & female \\
\hline Relationship status & defacto & single & married & single & defacto \\
\hline Dependents at home & 0 & 0 & 2 & 3 & 2 \\
\hline $\begin{array}{l}\text { Employment status - hours, } \\
\text { paid/unpaid/study }\end{array}$ & Full time health services & $\begin{array}{l}\text { Full time Computer and Computer } \\
\text { Peripheral Retailing }\end{array}$ & $\begin{array}{l}\text { Full time Management Advice and } \\
\text { Related Consulting Services }\end{array}$ & $\begin{array}{c}\text { Full time other social assistance } \\
\text { services }\end{array}$ & $\begin{array}{c}\text { Full time Professional Photographic } \\
\text { Services }\end{array}$ \\
\hline Ethnicity & NZ European & NZ European & NZ European & NZ European & NZ European \\
\hline Self-reported diagnosis & fibromyalgia & juvenile arthritis, psoriatic arthritis & $\begin{array}{c}\text { osteoarthritis, bilateral hips, knees, } \\
\text { ankles }\end{array}$ & nonspecific low back pain & osteoarthritis, fibromyalgia \\
\hline \multicolumn{6}{|c|}{ Pain Activities Relation Questionnaire } \\
\hline Contingency & 3.86 & 4.29 & 4.00 & 4.86 & 4.29 \\
\hline Avoidance & 2.75 & 2.63 & 1.75 & 2.00 & 1.88 \\
\hline Pacing & 3.50 & 2.83 & 0.83 & 3.83 & 3.17 \\
\hline Confronting & 3.00 & 4.14 & 4.00 & 3.43 & 3.43 \\
\hline \multicolumn{6}{|c|}{ Chronic Pain Acceptance Questionnaire } \\
\hline Willingness & 23 & 22 & 24 & 16 & 22 \\
\hline Activity engagement & 22 & 19 & 24 & 18 & 21 \\
\hline \multicolumn{6}{|c|}{ Chronic Pain Coping Inventory } \\
\hline Guarding & 12.14 & 10.29 & 12.00 & 11.14 & 17.29 \\
\hline Resting & 7.40 & 7.60 & 8.40 & 5.40 & 9.40 \\
\hline Asking for assistance & 9.00 & 9.50 & 14.50 & 9.00 & 15.50 \\
\hline Exercise/stretch & 21.20 & 20.00 & 21.20 & 23.80 & 12.00 \\
\hline Relaxation & 25.20 & 22.20 & 24.20 & 25.20 & 24.20 \\
\hline Task persistence & 23.83 & 27.00 & 26.00 & 27.00 & 27.00 \\
\hline Coping self-statements & 10.60 & 16.60 & 12.60 & 16.60 & 22.00 \\
\hline Seeking social support & 21.20 & 24.00 & 17.60 & 20.00 & 21.00 \\
\hline \multicolumn{6}{|c|}{ Profile of Chronic Pain - Screen } \\
\hline Severity & 17.00 & 18.00 & 20.00 & 22.00 & 18.00 \\
\hline Interference & 5.00 & 2.00 & 4.00 & 4.00 & 3.00 \\
\hline Emotional burden & 3.00 & 2.00 & 5.00 & 2.00 & 3.00 \\
\hline
\end{tabular}




\begin{tabular}{|c|c|c|}
\hline Study Number4 & 16 & 17 \\
\hline Age & 48 & 19 \\
\hline Gender & male & female \\
\hline Relationship status & married & single \\
\hline Dependents at home & 1 & 0 \\
\hline Employment status - hours, paid/unpaid/study & Full time higher education & Full time Higher Education \\
\hline Ethnicity & HongKong Chinese & NZ Maori \\
\hline Self-reported diagnosis & migraine, abdominal pain & widespread pain, ?fibromyalgia \\
\hline \multicolumn{3}{|l|}{ Pain Activities Relation Questionnaire } \\
\hline Contingency & 4.14 & 3 \\
\hline Avoidance & 2.00 & 2.5 \\
\hline Pacing & 4.67 & 3.5 \\
\hline Confronting & 2.71 & 1.71 \\
\hline \multicolumn{3}{|l|}{ Chronic Pain Acceptance Questionnaire } \\
\hline Willingness & 22 & 22 \\
\hline Activity engagement & 24 & 23 \\
\hline \multicolumn{3}{|l|}{ Chronic Pain Coping Inventory } \\
\hline Guarding & 15.14 & 12.00 \\
\hline Resting & 4.40 & 3.20 \\
\hline Asking for assistance & 11.00 & 9.00 \\
\hline Exercise/stretch & 11.00 & 12.00 \\
\hline Relaxation & 25.20 & 24.20 \\
\hline Task persistence & 29.00 & 27.00 \\
\hline Coping self-statements & 20.00 & 18.80 \\
\hline Seeking social support & 21.00 & 20.80 \\
\hline \multicolumn{3}{|l|}{ Profile of Chronic Pain - Screen } \\
\hline Severity & 23.00 & 23.00 \\
\hline Interference & 4.00 & 6.00 \\
\hline Emotional burden & 5.00 & 4.00 \\
\hline
\end{tabular}


Thompson, B. Living Well with Chronic Pain: A grounded theory Appendix 3 - Participants 